\title{
The Involvement of Interhemispheric Interaction In Developmental Dyslexia
}

By

\author{
Hiroko Sotozaki
}

A thesis submitted to the Faculty of Graduate Studies and Research in partial fulfillment of the requirements for the degree of

Doctor of Philosophy

Department of Psychology

Carleton University

Ottawa, Ontario

April, 2004

(C) 2004, Hiroko Sotozaki 


\author{
National Library \\ of Canada \\ Acquisitions and \\ Bibliographic Services \\ 395 Wellington Street \\ Ottawa ON K1A ON4 \\ Canada
}

Bibliothèque nationale

du Canada

Acquisisitons et services bibliographiques

395 , rue Wellington Ottawa ON K1A ON4 Canada
Your file Votre référence ISBN: 0-612-94217-1

Ourfile Notre référence ISBN: 0-612-94217-1
The author has granted a nonexclusive licence allowing the National Library of Canada to reproduce, loan, distribute or sell copies of this thesis in microform, paper or electronic formats.

The author retains ownership of the copyright in this thesis. Neither the thesis nor substantial extracts from it may be printed or otherwise reproduced without the author's permission.
L'auteur a accordé une licence non exclusive permettant à la Bibliothèque nationale du Canada de reproduire, prêter, distribuer ou vendre des copies de cette thèse sous la forme de microfiche/film, de reproduction sur papier ou sur format électronique.

L'auteur conserve la propriété du droit d'auteur qui protège cette thèse. $\mathrm{Ni}$ la thèse ni des extraits substantiels de celle-ci ne doivent être imprimés ou aturement reproduits sans son autorisation.
In compliance with the Canadian Privacy Act some supporting forms may have been removed from this dissertation.

While these forms may be included in the document page count, their removal does not represent any loss of content from the dissertation.
Conformément à la loi canadienne sur la protection de la vie privée, quelques formulaires secondaires ont été enlevés de ce manuscrit.

Bien que ces formulaires aient inclus dans la pagination, il n'y aura aucun contenu manquant. 


\begin{abstract}
This thesis investigated two hypotheses: (1) whether developmental dyslexia is associated with inefficient interhemispheric communication and (2) whether DD is a specific reading disorder. Participants (56 males, 15 females) were categorized into four groups: (1) a developmental dyslexic (DD) group ( $\mathrm{n}=19$, mean age of 13.09 years); (2) a chronological age-matched (CA) group ( $n=25$, mean age of 13.04 years); (3) a CA poor reader (CAP) group ( $n=10$, mean age of 13.09 years); and (4) a young age (YA) group $(\mathrm{n}=19$, mean age of 9.01 years). Experiment 1 (Finger localization), Experiments 2 (Rhyming judgement) and 3 (Primed lexical decisions) investigated efficiency in interhemispheric communication using different tasks. In Experiment 1, accurate responses were compared in the Crossed and Uncrossed conditions. The results showed that all four groups had lower scores in the Crossed than the Uncrossed conditions, suggesting that interhemispheric communication degrades tactile information. The results also demonstrated that the DD, CAP, and YA groups had significantly lower scores than the CA group in the Crossed condition, suggesting that inefficient interhemispheric communication may be common in developmental dyslexia and general poor reading ability. In Experiment 2 and 3, the RT and accuracy were compared in the Bilateral and Unilateral conditions using a visual half-field presentation paradigm. In Experiment 2, a 3-way interaction of Condition $x$ Visual Field $x$ Group revealed that the DD group responded more slowly and less accurately, while the CAP group responded faster and more accurately in the Bilateral than the Unilateral Condition. In Experiment 3, the main effect of Stimulus Pairs revealed priming effects in the DD group but not in the CAP group, suggesting that the DD group had an intact mental lexicon. This priming effect
\end{abstract}


clearly dissociated these two groups. In conclusion, the results from the three Experiments suggest that dyslexia is associated with inefficient interhemispheric communication, and that DD is qualitatively different from poor reading ability. 


\section{Acknowledgements}

This thesis was completed with the support and encouragement of many people. Primarily, I wish to thank my supervisor, Dr. Shelley Parlow, for her time and supervision throughout the preparation, especially toward the end of the preparation process, when she "pushed me to the limit" to improve this thesis as much as possible. Secondarily, I thank Ms. Linda ONeil for making herself so available and providing excellent suggestions throughout the long editing process.

Further, I wish to thank the principal of the Saganoska Demonstration School, Ms. Lindi Pierce, and her staff, for arranging and supporting the testing of students as well as for making me feel at home during my several visits to the school. I also thank the principals and staff of other Provincial Demonstration Schools (Amethyst School and Trillium School) for warmly welcoming me to their schools. I am particularly grateful to the principal of the Henry Larsen Elementary School of the Ottawa-Carleton District School Board, Mr. Denis Landry, for understanding my difficulties in recruiting students and graciously allowing me to test students over two school terms.

I also thank Sr. Lillian Clark, who has devoted herself to dyslexia education for over 10 years, for allowing me to observe her classes for dyslexic students at the initial stage of this thesis project and for her constant encouragement throughout the long process. I am also grateful to the Learning Disabilities Association-Ottawa, Ontario for helping me with recruitment. 
I wish to thank Dr. Chris Herdman and Dr. Jo-Anne LeFevre for introducing me to how fascinating the study of cognitive psychology can be(!), when I was an undergraduate student.

I would like to extend my appreciation to the parents who gave permission for their children to participate in this thesis project, and their children who gave their best performance, particularly if they found the experimental tasks hard or boring!

And lastly, I wish to thank my children, Yuichiro and Misako, my friend, Yuko in Japan, and my mother, who passed away two years ago, for inspiring me to find the immense energy to keep moving forward. In particular, without my mother's emotional and financial support since our arrival in Canada 14 years ago, our family could not have survived here, and needless to say, I could not have completed this thesis. 


\section{Table of Contents}

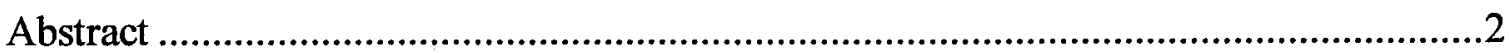

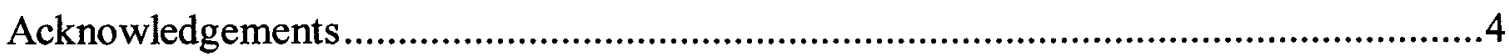

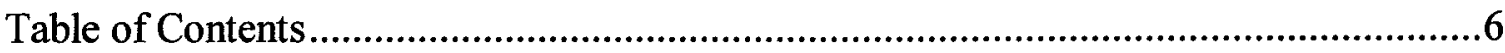

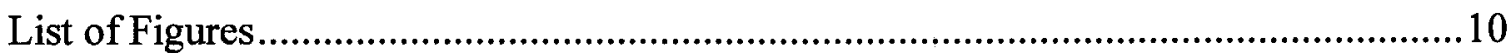

List of Tables

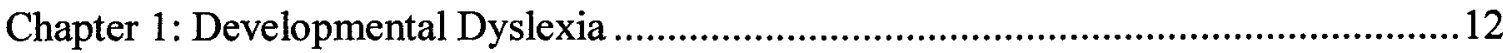

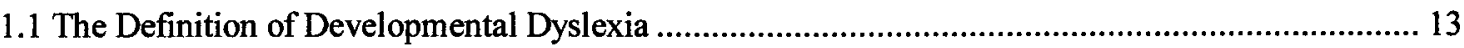

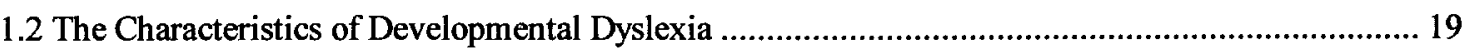

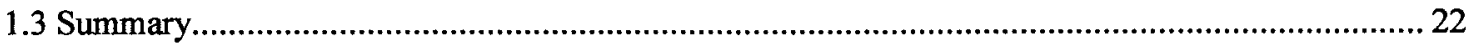

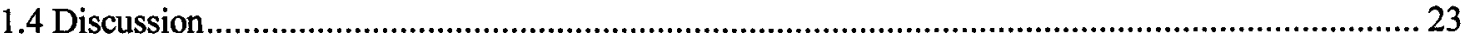

Chapter 2: The Phonological Deficit Theory ........................................................25

2. 1 Phonological Awareness and Developmental Dyslexia ................................................................ 27

2. 2 Phonological Recoding and Developmental Dyslexia ..................................................................... 31

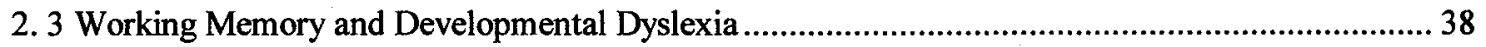

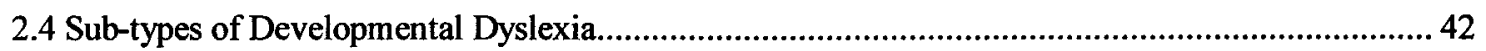

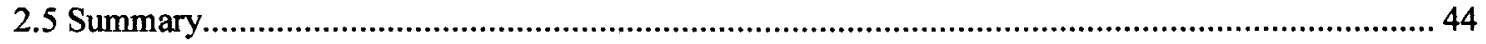

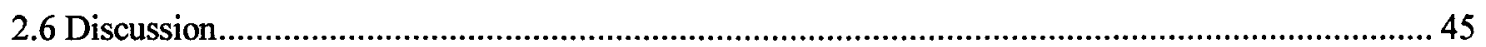

Chapter 3: The Left Hemisphere Dysfunction Theory ...........................................47

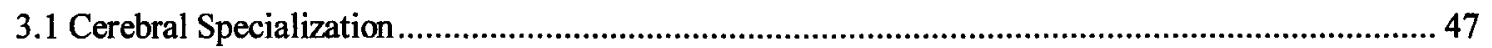

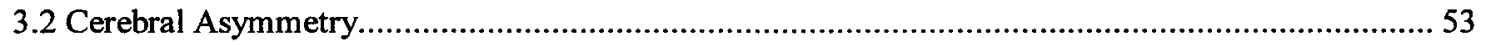

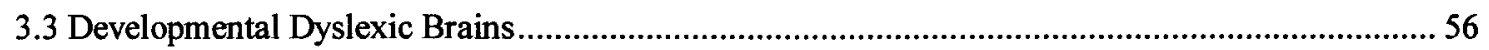

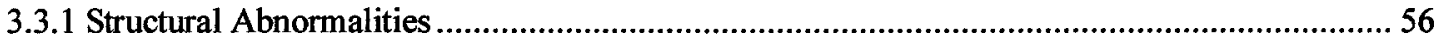

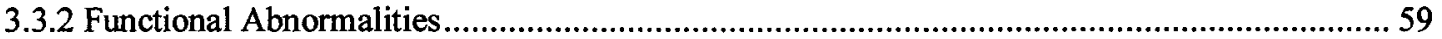

3.4 Summary 
3.5 Discussion 63

Chapter 4: The Magnocellular Deficit Theory .66

4.1 Description of the Magnocellular and Parvocellular Pathways....................................................66

4.2 The Controversy Surrounding the Magnocellular Deficit Theory …............................................... 70

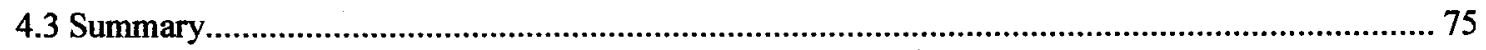

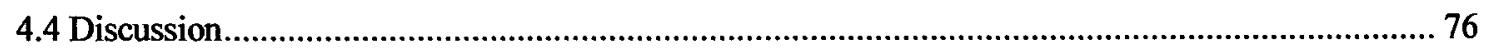

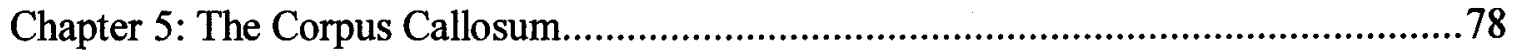

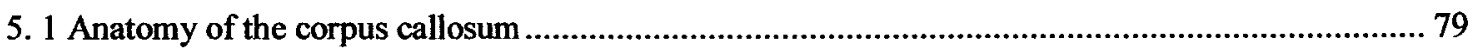

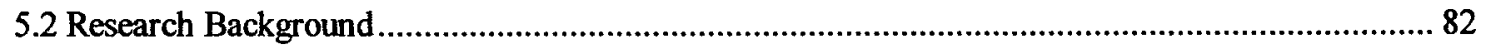

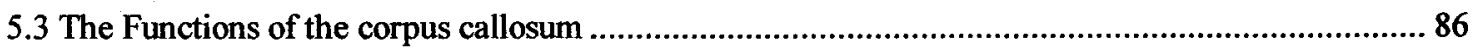

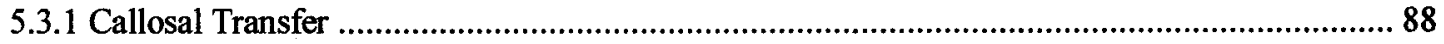

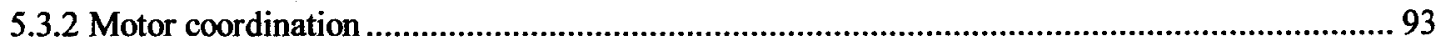

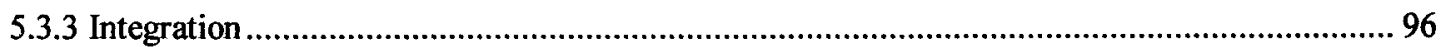

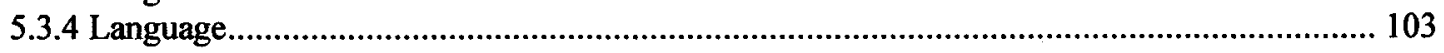

5.4 Callosal Size, Gender, and Handedness....................................................................................... 105

Chapter 6: The interhemispheric disconnection theory ......................................111

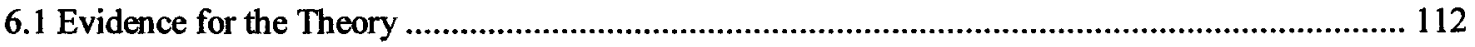

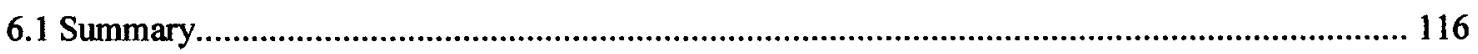

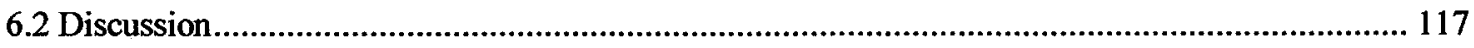

Chapter 7: Evaluation of the four main theories................................................ 119

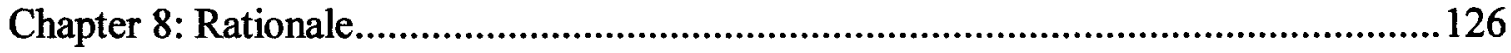

8.1. Investigation the Interhemispheric Disconnection Theory........................................................ 127

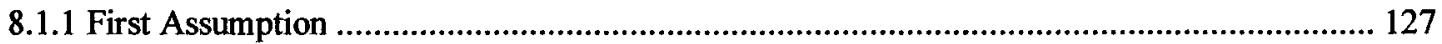

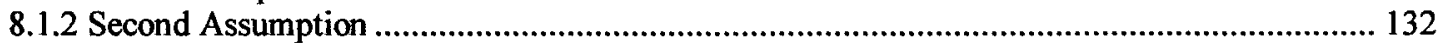

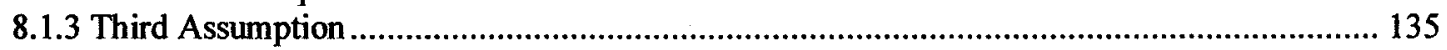

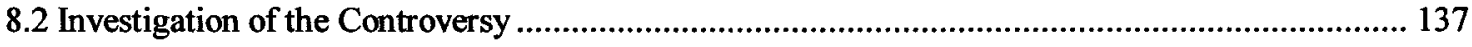

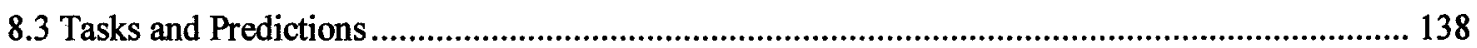

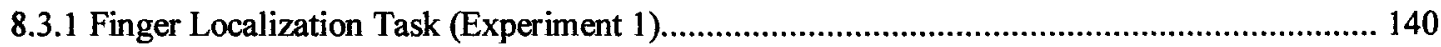

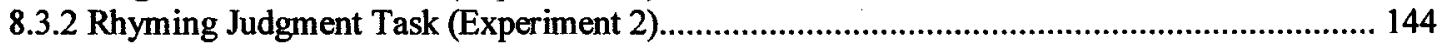

8.3.3 Primed Lexical Decision Task (Experiment 3) ....................................................................... 147 


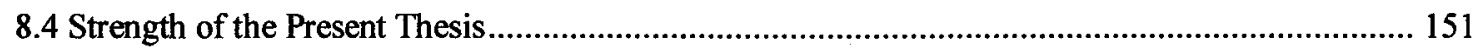

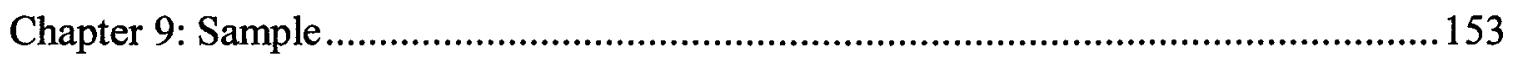

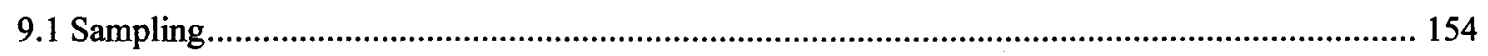

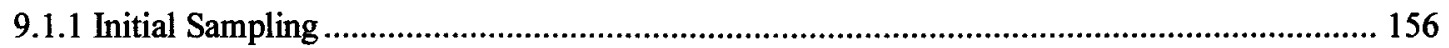

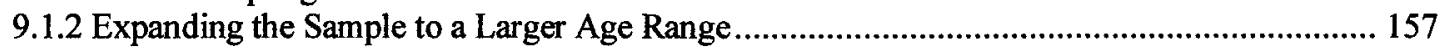

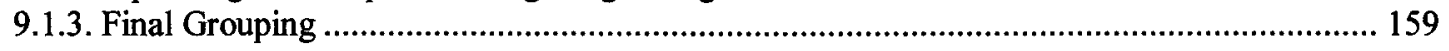

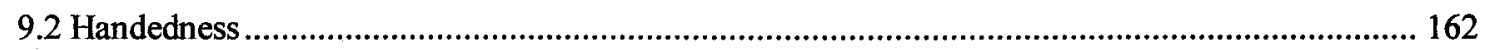

9.3 Verbal and Non-Verbal Assessment Materials ..................................................................... 163

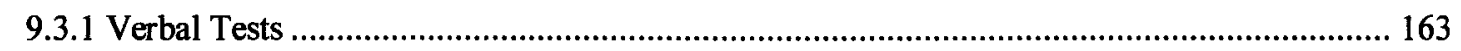

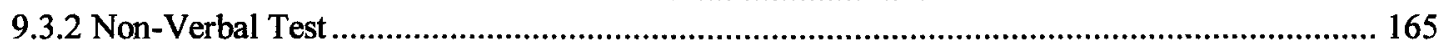

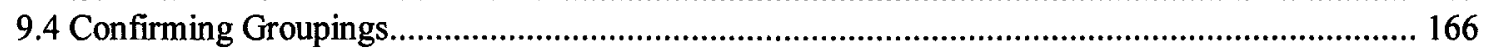

9.5 Investigation of Sub-types of Developmental Dyslexia ........................................................... 168

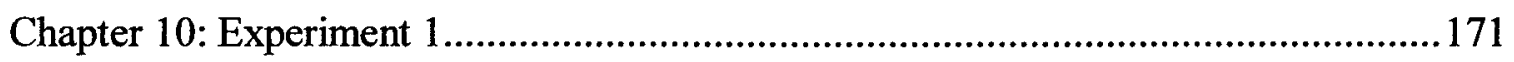

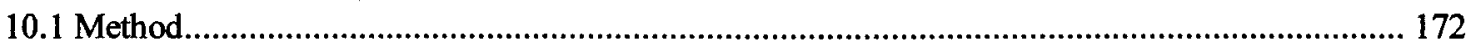

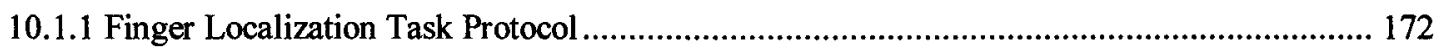

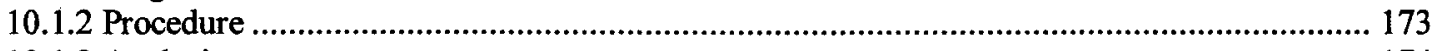

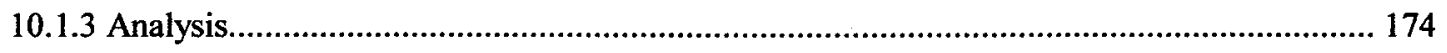

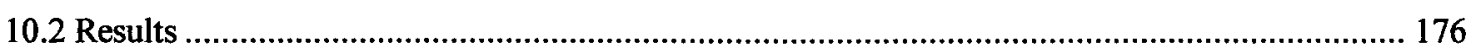

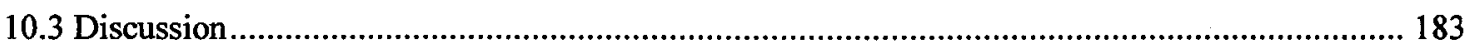

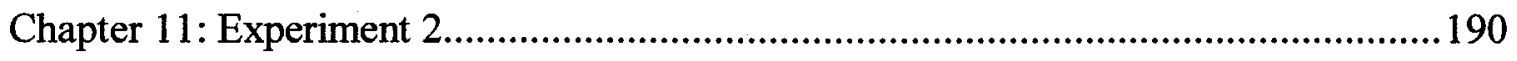

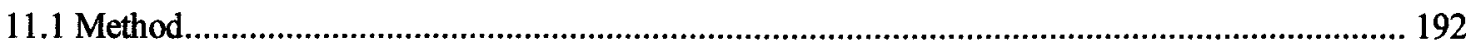

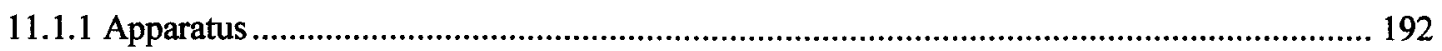

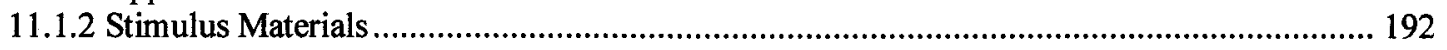

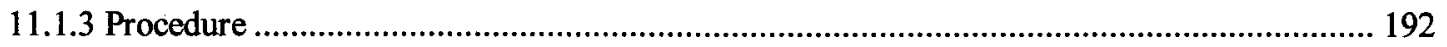

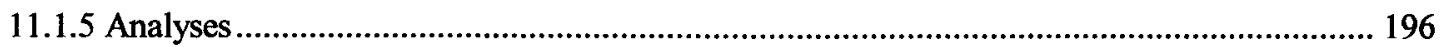

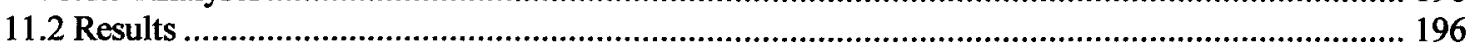

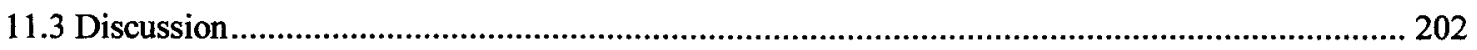

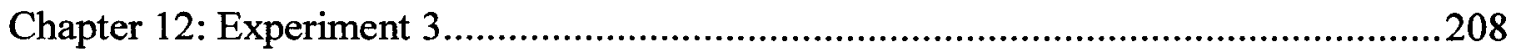

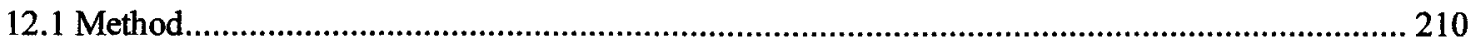

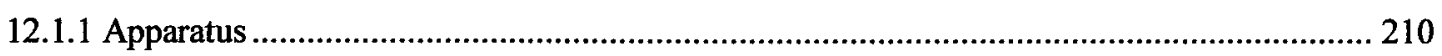

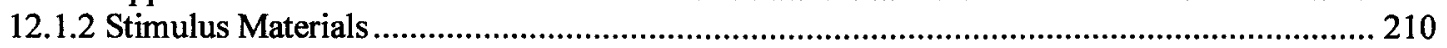

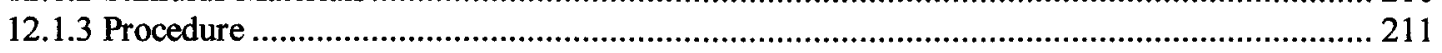

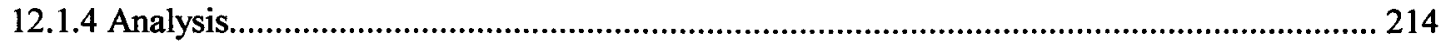




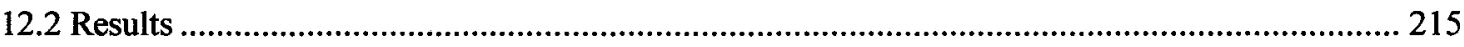

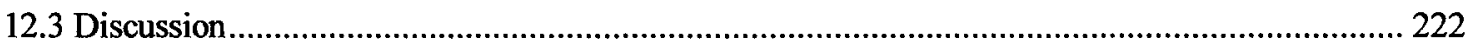

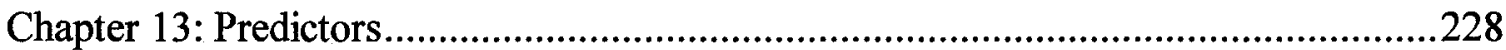

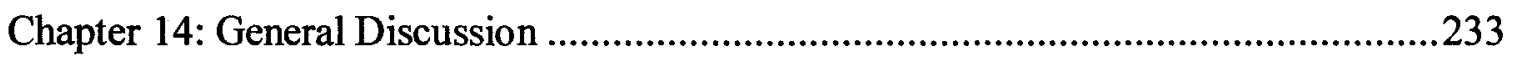

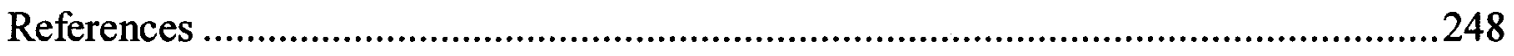

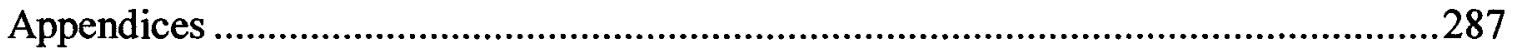




\section{List of Figures}

Figure 1.1. Schematic Representation of Orton's Theory 15

Figure 3.1. Reading Pathways Based on Alexia Research. 49

Figure 3.2. Computer Axial Tomogram (CT) of a Brain Shows the Usual Pattern of Hemispheric

Asymmetry. 53

Figure 3.3. Anatomical Landmarks and Typical Left-Right Differences. 54

Figure 4.1. The Visual Pathways From the Retinal to the Primary Visual Cortex Through the Thalamus 67

Figure 4.2. Parallel Visual Pathways of the Magnocells and Parvocells ..........................................................68

Figure 5.1. The Position of the CC Compared wWith the Locations of Other Brain Commissures. .................. 79

Figure 5.2. Diagram of the Midsagittal View of the $\mathrm{CC}$ of the Human Adult................................................. 81

Figure 5.3. Visual Pathways to the Cerebral Hemispheres.............................................................................. 85

Figure 5.4. Illustration of the Crank-Handles. ........................................................................................... 94

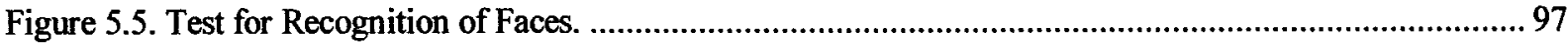

Figure 5.6. Appearance-Function Matching Chimeric Pictures Test ............................................................... 99

Figure 5.7. Composite fMRI images of the distribution of activation in a rhyme judgement task.................... 107

Figure 9.1. The Results of the Test of Word Reading Efficiency (TOWRE) …............................................. 168

and the Test of Nonverbal Intelligence-3 (TONI-3) in the Four Groups........................................................ 168

Figure 10.1. Mean Accuracy and Standard Errors for 2-Finger, 3-Finger, and 4-Finger Sequences.................. 180

Figure 10.2. Mean Accuracy and Standard Errors of Crossed and Uncrossed Difference. ............................. 181

Figure 11.1. Procedure For the Rhyming Judgment Task. .......................................................................... 195

Figure 11.2. The interaction of Group $x$ Visual Field $x$ Condition in Reaction Time. ....................................... 198

Figure 11.3. The Interaction of Group $x$ Visual Field $x$ Condition in Accuracy............................................ 199

Figure 12.1. Procedure For the Lexical Decision Task. .......................................................................... 212

Figure 12.2. The Interaction of Group $x$ Stimulus Pair in RT. .................................................................. 218

Figure 12.3. The Interaction of Group $x$ Stimulus Type in Accuracy.......................................................... 219 


\section{List of Tables}

Table 9.1

List of Recruitment Sources and Distribution of Students

Table 9.2

Profile of an Experimental Group and Control Groups

Table 10.1

Mean Percentage Correct Responses and Sequence Errors for Four Groups

Table 10.2

Mean percentage of sequence errors for four groups.

Table 10. 3

Mean Percentage of Crossed-Uncrossed Difference for Four Groups

Table 10.4

Correlations of Finger Localization Task Scores, Age, Standardized TONI-3and TOWRE Scores

Table 11.1

Group Means of Reaction Times (msec) and Percentage of Correct Responses for Each of Four Groups........

Table 11.2

202

Correlations of Phonological Task Scores with Age, TONI-3, and Sub-tests of TOWRE Scores

Table 12.1

Group Means of Reaction Time and Percentage of Correct Responses

Table 12.2

Correlations of Semantic Task Scores with Age, TONI-3, and TOWRE Scores

Table 13.1

Experimental Tasks of the Three Experiments

Table 13.2

Results of Tests of Regression Coefficients 


\section{Chapter 1: Developmental Dyslexia}

Reading is valued in modern society (Snowling, 2000). Most children are expected to acquire reading skills with adequate education and age. Some children, however, experience unexpected severe difficulty in learning to read despite normal intelligence, adequate educational opportunities, and the lack of any physical or mental disorder. There seems to be no particular reason as to why these children are unable to develop reading skills. Most of them show difficulty in learning phonetic or phonological rules for distinguishing regular words, such as hat, bike, test (Ellis, 1994). This reading disability is development dyslexia (DD). It has been estimated that 3-10\% of all school children/students suffer from DD (Rutter \&Yule, 1975; Snowling, 2000). Though over a century has passed since the first case of DD was reported in the British Medical Journal (Morgan, 1896), the etiology of DD remains speculative and a simple definition of DD has not been widely accepted.

Many theories have been put forward to account for developmental dyslexia, each focusing on different cognitive or brain functions. The current main theories focus on poor phonological processing, dysfunction of the language areas in the left hemisphere, and deficits in visual systems. This thesis allocates one chapter to the discussion of each theory: (1) the phonological deficit theory (Chapter 2), (2) the left hemispheric dysfunction theory (Chapter 3), and (3) the magnocellular deficit theory (Chapter 4). According to a traditional view (Geschwind \& Galaburda, 1985a, 1985b, 1985c; Sperry, Gazzaniga, \& Bogen, 1969), the left hemisphere has a main role in reading processing. This traditional view is challenged by the interhemispheric disconnection theory 
(Chapter 6), which focuses on interhemispheric interaction in reading processing through the corpus callosum, the largest fiber bundle connecting the two cerebral hemispheres (Banich \& Karol, 1992; Chiarello, 1995). Although there has been much research effort to understand developmental dyslexia, up to now none of the theories has succeeded in providing a full account for developmental dyslexia.

The present thesis explores the interhemispheric disconnection theory. It focuses on three different callosal regions that have been implicated in DD. Before discussing the theory, the structure and functions of the corpus callosum are discussed in Chapter 5 . In Chapter 7 , the four theories are evaluated, followed by the rationale for the present project in Chapter 8. Sampling and grouping procedure for the present project are described in Chapter 9. The three experiments conducted for this project are separately described including their methods, results and discussion, in Chapter 10 (Experiment 1), Chapter 11(Experiment 2) and Chapter 12 (Experiment 3), followed by a short chapter presenting data for the best predictors of standardized reading scores (Chapter 13). In

Chapter 14, the general discussion of the three experiments is presented. But first, the definition of DD is described with a brief overview of the history of this research followed by a description of the accepted characteristics of DD and introduction of important issues in DD research.

\subsection{The Definition of Developmental Dyslexia}

The first cases of DD were called 'congenital word-blindness'. The name reflected a comparison with adult patients suffering from 'word-blindness' (Kussmaul, 1877), i.e., an acquired inability to read words resulting from brain trauma, despite sight and speech 
being preserved intact (Hinshelwood, 1907). Hinshelwood, a British ophthalmologist, analyzed and described the cases of four DD children and summarized his findings in a 1917 book entitled, Congenital Word-Blindness. He pointed out that children with congenital word blindness had difficulty in reading and writing despite normal intelligence and adequate social backgrounds, and suggested that the difficulty might stem from cerebral problems rather than visual problems. Thus, from the first dyslexic cases, it was assumed that DD was a complex disorder, and might involve the higher brain functions.

In North America, Samuel T. Orton, a physician, promoted the concept of 'sterephosymbolia' in his 1937 book, Reading, Writing and Speech Problems in Children. In his practice, Orton observed that children with reading problems tended to read words backwards or to write in mirror images. He interpreted these phenomena as reflecting incomplete cerebral dominance. Cerebral dominance is the idea that one of the brain hemispheres, in most cases the left hemisphere, suppresses the other, particularly as it applies to language. Orton argued that visual information is normally represented in a mirror image fashion in the left and right hemispheres, and that a lack of sufficient development of dominant hemisphere for language would cause confusion in reading and writing (Figure 1.1). Orton's term and concept is no longer used to describe developmental dyslexia, but the basic notion that a problem of absent or inadequate hemispheric dominance for important cognitive functions related to reading underlying DD is still under investigation today. 


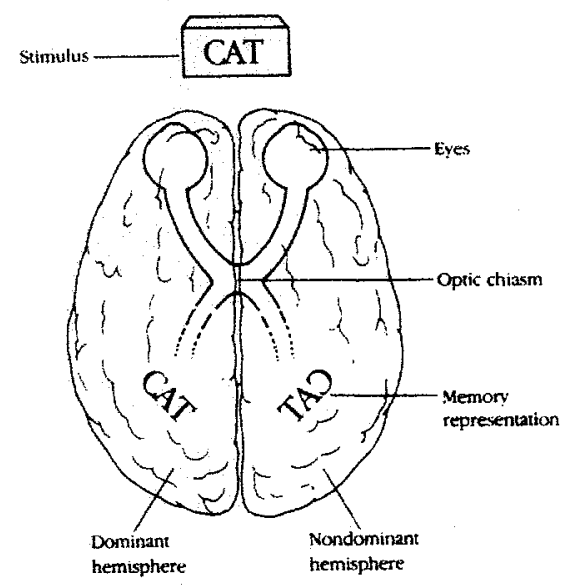

Figure 1.1. Schematic representation of Orton's theory.

Orton assumed that a visual stimulus is represented in opposite orientations in the two hemispheres. (Corballis, 1974).

A 'balance' model developed by Bakker $(1979,1992)$ attempted to incorporate and expand on some of Orton's ideas. This model postulated that early and advanced reading are primarily mediated by the right and left cerebral hemispheres, respectively, and thus, it is necessary to shift reading strategies from the right to the left hemisphere with advancing reading skills. The balance model, therefore, suggests that a failure in shifting strategies leaves children with right hemisphere $(\mathrm{RH})$ strategies (e.g., relying on visual features), and prohibits them from developing more effective left hemisphere (LH) strategies (e.g., relying on phonological knowledge). Based on this theory, Bakker classified dyslexic children into"two types: P-type and L-type. P-type dyslexia develops when children fail to shift reading strategies from the $\mathrm{RH}$ to the $\mathrm{LH}$ and remain focused on the perceptual features of the text. L-type dyslexia develops when children rely prematurely on left-hemispheric linguistic strategies in the early stages of reading 
development. Hynd (1992) criticized Bakker's balance model because it did not explain the main concept of how 'imbalance' or 'shift' occurs. Hynd also pointed out that the involvement of the $\mathrm{RH}$ in early reading in normal children has not been proven and that no researcher has replicated Bakker's conceptualization of the two dyslexic subtypes.

It was becoming clear that reading difficulty can be caused by many factors, including an inadequate educational environment, low IQ, or attentional deficits. By the 1970 's, a clear definition for DD was required. The World Federation of Neurology (Critchley, 1970) set the definition for DD thus: "Dyslexia is a disorder manifested by difficulty in learning to read despite conventional instruction, adequate intelligence and sociocultural opportunity. It is dependent upon fundamental cognitive disabilities which are frequently of constitutional origin". Ellis (1994) pointed out that this definition was not clear as to what 'adequate intelligence' referred to or what 'constitutional origin' was. In the last 30 years, clinical psychologists, educators and researchers studying DD have usually adopted a set of criteria requiring that a child have an IQ of 90 or above, a reading age at least two years behind the child's chronological age group, adequate opportunity to learn to read, no impairment in hearing or vision, and no obvious neurological disorder. The main criterion emphasized here is the discrepancy between IQ and reading ability. This criterion is based on the assumption that children within normal IQ range are expected to develop normal reading skills without undue difficulty, and, thus, if children with normal IQs show difficulty in acquiring reading skills, the reading difficulty is unexpected. Thus, DD can be summarized as 'unexpected reading difficulty' (Ellis, 1994; Snowling, 2000). 
This lack of a clear definition for DD reflects on the wide range of prevalence: approximately 3-10\% of all school-age children (Rutter \& Yule, 1975). The wide range is because the prevalence of DD depends on the cut-off point of the discrepancy between IQ and expected reading accuracy, which is always arbitrary (Snowling, 2000). For example, Yule, Rutter, Berger and Thompson (1974) reported a prevalence of DD ranging from $3.1 \%$ among 10 -year-olds in the Isle of Wight to $6.9 \%$ in London based on a reading-IQ discrepancy criterion.

The traditional reading-IQ discrepancy was challenged by some researchers (Fletcher, Stuebing, Shaywitz et al., 1994; Stanovich, 1991, Stanovich \& Siegel, 1994). The main argument was that there was no empirical evidence to differentiate between poor readers with high IQ (dyslexic readers) and with low IQ (general poor readers). The general poor readers can be defined as those whose IQ and standardized reading test scores are below the average, i.e., there is little difference between the two scores. For example, a study with normal children (Stanovich, Cunningham \& Feeman, 1984) found that IQ and reading test scores had a low correlation with a phonological awareness test and nonword reading. Although genetic linkage studies (DeFries, Fulker, \& LaBuda, 1987; DeFries, Gillis, \& Wadsworth, 1993, Galaburda, Sherman, Glenn, Aboitiz, \& Geschwind, 1985; Larsen, Höien, Lundberg \& Odegaard, 1990; Smith \& Goldgar, 1986) have provided causation for some DD cases, there was criticism that these cases were conducted based on a discrepancy criterion in defining DD cases. Stanovich argued (1994) that there was no evidence available as to whether genetic linkage similar to the DD cases would be found in general poor readers. In addition, he argued that poor nonword reading equivalent to that of DD children was also found in general poor 
readers (see also Felton \& Wood, 1992; Siegel \& Ryan, 1988; Stanovich \& Siegel, 1994). Stanovich (1994) concluded that there was no indication that the nature of reading processing within the word recognition domain differs for DD children from general poor readers. Thus, some researchers denied the existence of qualitatively different reading difficulty from general reading difficulty. Instead, they argued that reading difficulty exists in a continuum, and DD children lie at the edge of poor readers in the normal distribution.

The controversy around defining DD has not yet been resolved. Developing a definition for $\mathrm{DD}$ is an important issue in dyslexia research. This raises a question as to whether DD children qualitatively differ from general poor readers.

In summary, over one hundred years ago in England, the first DD cases (1917) were called 'congenital word-blindness', associated with 'word-blindness' caused by brain trauma. In North America, Orton (1937) promoted the concept of sterephosymbolia, which was thought to be due to incomplete cerebral dominance. Bakker $(1979,1992)$ developed a 'balance' model to expand some of Orton's ideas. The model suggests that a failure in shifting strategies from the RH to the LH leaves children with visual strategies. The balance model was criticized by Hynd (1992) in that the RH involvement in early reading has not been proven. Although the World Federation of Neurology (1970) set the definition for DD, it was criticized because of its ambiguous definition. Thus, in practice, clinicians, educators and researchers have adopted a set of criteria focusing on a readingIQ discrepancy. Though this traditional definition has been used for over 30 years, it has been criticized by some researchers who claimed that dissociation between dyslexic and general poor readers based on a reading-IQ discrepancy is not valid. They argue that DD 
children do not qualitatively differ from general poor readers. A conclusive definition for DD has not yet been established.

\subsection{The Characteristics of Developmental Dyslexia}

The main impairment in DD is obviously reading difficulty. The source of this difficulty varies, however. Most DD children have difficulty in phonological processing. This is reflected in reading novel and regular words (e.g., cake, date, state) using grapheme-phoneme conversion (GPC) rules. The deficit is most apparent when these children read novel, unfamiliar words and nonwords (e.g., brane, zink) (Bryant \& Impey, 1986; Ellis 1994; Snowling, 1995). A small number of DD children (less than $20 \%$ of DD) appear to have more trouble with visual processing than phonological processing. Thus, the main problem lies more in reading irregular words (e.g., yacht, pint, island) than in reading regular words. These children tend to apply GPC rules to read irregular words (e.g., come ->kome, island -> izland) (Coltheart, Masterson, Bying, Prior, Riddoch, 1983; Temple \& Marshall, 1983). Since there are few pure cases that demonstrate only either linguistic deficit of the subtype, and many dyslexic children show problems in reading both regular and irregular words (Seymour, 1986; Snowling, 1987), the existence of subtypes of DD is controversial. Subtypes of DD are further discussed in Chapter 2.4 .

Besides the linguistic deficits, other researchers have focused on general deficits in cognitive abilities underlying reading and other complex tasks, such as attention (Hynd, Semrud-Clikeman, Lorys, Novey, \& Eliopulos, 1990), short term memory (e.g., repeating simple sentences) (Macaruso et al., 1995), long term memory (e.g., object 
naming) (Swan \& Goswami, 1997), sequential processing (e.g., counting numbers, telling time or years) (Bakker, 1973), and spatial processing (e.g., telling right or left) (Ellis, 1994). These cognitive impairments are mildly and unevenly shown in DD children. They are also seen in poor general readers with low IQs, and therefore, they are not specific to DD (Snowling, 1987).

Regarding the etiology, autopsy and magnetic resonance imaging (MRI) studies (Drake, 1968; Galaburda et al., 1985; Larsen et al., 1990) have shown that many, but not all, DD brains are abnormally organized with cell anomalies located particularly in the language area in the left hemisphere (LH). Developmental dyslexia tends to run in families, suggesting that a genetic component may be involved in DD (Grigorenko, Wood, Meyer, \& Pauls, 2000; Oppenheim, Skerry, Tramo, \& Gazzaniga, 1989). Since boys are diagnosed with DD three times as frequently as girls, some researchers (e.g., Galaburda et al., 1985) have speculated on the involvement of a hormonal imbalance involving testosterone in DD. Left-handers are also more often seen than right-handers among DD children (Ellis, 1994), implying some association with brain lateralization or cerebral hemispheric specialization (Witelson, 1989). In addition, many DD children complain of transporting letters or words (e.g., $d->$ b or saw $->$ was). This complaint suggests that DD may be caused by a specific deficit associated with visual processing. In fact, much research found that about $75 \%$ of DD children have deficits in the magnocellular system in visual processing (Stein, \& Walsh, 1997; Lovegrove, Martin, \& Slaghuis, 1986).

Furthermore, DD children frequently demonstrate poor motor processing including slowness and clumsiness, which are not directly associated with reading. The 
slowness can be readily demonstrated in timed color or object naming tasks (Denkla, 1974; Denkla \& Rudel, 1976; McBride-Chang \& Manis, 1996), in which children are simply required to repeat colors or object names given a list of pictures (i.e., red, yellow, blue, green or umbrella, car, comb, telephone, scissors). The clumsiness is manifested in poor bimanual coordination in most (Gladstone, Best, \& Davidson, 1989), but not all, DD children (Gross-Glenn, \& Rothenberg, 1984). These diverse and unevenly distributed characteristics in DD suggest that DD might be composed of heterogeneous groups.

The heterogeneous characteristics of DD further suggest that multiple factors are involved in DD. Although a theory for DD requires explaining these diverse characteristics, each of the current major theories tends to focus on a single factor and leave some characteristics of DD unexplained. The three major theories discussed in this thesis are (1) the phonological deficit theory focusing on linguistic deficits, (2) the left hemisphere dysfunction theory discussing brain abnormalities associated with the left hemisphere, and (3) the magnocellular deficit theory associated with deficits in the visual system. In contrast, the interhemispheric disconnection theory focuses on interhemispheric communication. It will be argued that this theory is the most promising because it can incorporate many of the diverse characteristics of DD.

In summary, the main characteristic of DD is a linguistic deficit. Most DD children show poor phonological processing, which is dramatically demonstrated as an inability to read nonwords. Some DD children demonstrate a lexical processing deficit rather than one of phonological processing, typically manifested in reading irregular words. Minor cognitive deficits are also demonstrated by most DD children including deficits in memory, attention, or sequential processing. Genetic predisposition is also 
evident. Boys are more frequently seen than girls among DD children. Many developmental dyslexics are left-handers. Autopsy and MRI studies show that many DD adults and children have abnormal brain organization in the left hemisphere. Poor motor skills are frequently evident as well. Current dyslexia theories tend to focus on a single deficit in developing the theory.

\subsection{Summary}

Since the first cases of DD were found, different theories have attempted to account for DD. The terms 'congenital word-blindness' (Hinshelwood, 1917) and 'strephosymbolia' (Orton, 1937) clearly implied that some type of brain dysfunction was thought to be responsible in the early research. Subsequently, the balance model (Bakker, 1979, 1992), assumed that abnormal development of cerebral dominance causes developmental dyslexia. The definition by the Word Federation of Neurology (Critchley, 1970) was criticized as being vague. Many DD researchers currently use a set of criteria focusing on a discrepancy between IQ and age expected reading ability. Despite all efforts to define developmental dyslexia, no conclusive definition has yet been established.

One reason that $\mathrm{DD}$ is difficult to define is the diverse characteristics among DD children. Even the linguistic deficits, which are the main characteristics of DD, have been differently demonstrated among them as sub-types (Bakker, 1992; Boder, 1971, 1973; Castle \& Coltheart, 1993). The minor cognitive impairments and behavioural features, such as clumsiness and slowness, are also not evenly shown. 


\subsection{Discussion}

One of the main problems in DD research is that there is no clear definition for DD. A lack of the definition raises a question as to whether DD is a specific reading disorder or it falls in a continuum of general poor readers. Both DD children and general poor readers have reading difficulty. Investigating in what aspects DD children are different from general poor readers and if there is some common ground between them will give us insight into understanding developmental dyslexia.

It is also important to take into account the diverse characteristics in developing a theory for DD. Not all DD children are boys and left handers (Witelson, 1989). Not all DD children demonstrate poor phonological processing and some exhibit lexical problems (Castle \& Coltheart, 1993). In addition, about $25 \%$ of DD children do not show a magnocellular deficit (Lovegrove, Martin, \& Slaghuis, 1986). Theories that have emerged in the past 30 years tend to focus on a single deficit demonstrated in developmental dyslexia. While a theory focusing on a single deficit of DD can explain some characteristics of DD, it leaves others unexplained. For example, the phonological deficit theory (Chapter 2), which is more widely accepted than other theories, has provided convincing evidence that poor phonological awareness and decoding skills (i.e., a lack of ability in reflecting phonetics on written words) might cause DD (Snowling, 1995). The theory, however, merely investigates the linguistic aspects, such as poor encoding and decoding of words, but does not provide any account of the common characteristics of developmental dyslexia, such as poor bimanual coordination. The left hemisphere dysfunction theory (Chapter 3), as another example, discusses only structural 
and functional abnormalities in DD separately, and disregards the links between them. The magnocellular deficit theory (Chapter 4) stresses a particular deficit in visual processing, and does not discuss even a role for the magnocellular system in reading. Considering that multiple factors might be involved in DD as discussed earlier, any theory based on the single factor approach naturally will have limited ability to account for DD. In contrast, the interhemispheric disconnection theory discussed in Chapter 6 accommodates multiple factors associated with interhemispheric interaction, implying that reading processing involves not only the $\mathrm{LH}$, but also the $\mathrm{RH}$ in some ways. This thesis will explore the two main issues: the multiple factor approach to DD and DD as a specific reading disorder.

First, the phonological deficit theory, which has been most widely accepted in dyslexia research, will be discussed in the next chapter. 


\section{Chapter 2: The Phonological Deficit Theory}

The phonological deficit theory holds that developmental dyslexia is a linguistic disorder specifically involving deficits in phonological processing (Bryant, \& Goswami, 1987, Ellis, 1991; Goswami \& Bryant, 1990; Gough \& Tunmer, 1986; Kats, 1986; Lundberg, 1989; Snowling, 1981; Snowling, 1995; Snowling \& Hulme, 1994; Vellutino, 1979). The importance of phonological processing in reading has been evident in the literature for some time (Ehri, 1991; Ellis, 1991; Foorman \& Liberman, 1989; Goswami \& Bryant, 1990; Gough, 1996; Hulme \& Snowling, 1992; Muter, Hulme, Snowling \& Taylor, 1998; Snowling, 1995; Wagner \& Torgesen, 1987). Phonological processing refers to the use of phonological information (i.e., the sound of one's own language) in written and oral systems (Wagner \& Torgesen, 1987). In reading, regular words need to be broken into phonemic segments (Bradley \& Bryant, 1983; Bryant, 1998; Kirtlery, Bryant, Maclean, \& Bradley, 1989; Hulme, Muter, \& Snowling, 1998, Liberman, Shankweiler, Fischer, \& Carter, 1974; Lundberg, 1989; Lundberg, Olofesson, \& Wall, 1980; Rack, Hulme, Snowling, \& Wightman, 1994; Treiman, 1985), mapped on the sounds (i.e., phonological representations) stored in the mental lexicon (i.e., mental dictionary) (Mann, 1986; Vandervelden \& Siegel, 1995), and all sounds blended to verbalize the word. While searching appropriate sounds for a word in the mental lexicon, the information needs to be maintained in short-term memory (STM) (Adams \& Gathercole, 1996; Baddeley, 1981; Breznitz, 1997; Gathercole \& Baddeley, 1990; Jorm, 1983; Mann \& Liberman, 1984; Muter, Hulme, Snowling, \& Taylor, 1998). 
Phonological processing can be divided into two stages: encoding and decoding (Snowling, \& Hulme, 1994). In the encoding stage, phonological awareness is a critical element related to segmenting a word into phonemes (sound units, e.g., $/ \mathrm{m} /, / \mathrm{b} /$ ). Phonological awareness refers to one's awareness of manipulating and using the phonology of one's language (Mattingly, 1972). It can be measured by many tasks, such as tapping out the number of sounds in a word, reversing the order of sounds in a word (e.g., top->pot, pool->loop), or blending isolated sounds to form a word (e.g., m-a-p >map) (Lekowicz, 1980; Wagner \& Togesen, 1987). In the decoding stage, two components are essential: access to the phonological representations (i.e., sounds) in the mental lexicon and verbal STM (Snowling, 1995). Developmental dyslexic children exhibit deficits in both the encoding and decoding. Thus, they show poor performance in segmentation tasks (Snowling, 1981), word retrieval (Katz, 1986; Swan \& Goswami, 1997), and immediate word recall (Kramer, Knee \& Delis, 2000). They also exhibit great difficulty in nonword (e.g., gop, mulp, phot) reading (Rack, Snowling, \& Olson, 1992). By definition, phonological representations for nonwords are not stored in the mental lexicon. Therefore, to read nonwords, phonological knowledge is required, i.e., broken down into segments and mapped on the phonological representations in the mental lexicon. The difficulty in reading nonwords is strong evidence that DD children have impairment in phonological processing.

This chapter will discuss the relationships between DD and phonological awareness, phonological recoding (phonological representation), and working memory (verbal STM). 


\section{1 Phonological Awareness and Developmental Dyslexia}

English orthography consists of alphabetic letter sequences. There are certain relationships between letter sequences and sounds. Therefore, reading English requires learning the relationships between sounds and letter strings. Most children gradually learn these sound-letter relationships in a series of developmental stages (Snowling, 1995). Frith (1985) proposed a stage theory that consists of three phases for the development of reading skills before children become fluent readers. Each stage is characterized by different reading strategies: (1) logographic skills, (2) alphabetic skills, and (3) orthographic skills. These reading stages are hypothesized to develop in strict sequential order. In the logographic stage, reading relies on the salient visual features of words, and words are understood like pictures. Children are unaware of linguistic relationships across words, and reading strategies refer to sight vocabulary. Using sight vocabulary strategies, three-year-old children can read as many as 120 words (Soderbergh, 1971). However, there are limitations on the extent to which vocabulary can be increased using this strategy. In the alphabetic stage, reading skills are characterized by the correspondence of graphemes and individual phonemes. For example, four- or five-year-old children can differentiate each sound of the alphabet (e.g., hill, pig, toy). Thus, the clear distinction between the two stages is that children in the alphabetic stage have grapheme-phoneme awareness (Frith, 1985; Liberman, Shankweiler, Liberman, Fowler, \& Fischer, 1977; Steven \& Bruce, 1994), but those in the logographic stage do not. A grapheme by phoneme decoding strategy allows children to read novel and nonsense words. However, reading skills are implicit, i.e., children at 
this stage are not aware of phonological relationships. In contrast, reading skills of children at the orthographic stage become morphological (able to recognize the smallest unit of linguistic meaning, e.g., un-happy) and analytic. The main feature of their reading strategy is that it allows the utilization of orthographic units in reading. That is, regular words can be read by grapheme-phoneme conversion (GPC) rules (e.g., cat -> sat, beach $->$ peach, station->expectation), while irregular words are identified by a different strategy, such as whole word recognition (e.g., yacht, comb). The critical distinction between the logographic and orthographic stages is, however, that children at the orthographic stage can segment an irregular word into syllables for memorizing (e.g., yacht, com-b) rather than memorizing the whole irregular word as a picture at the logographic stage (e.g., yacht, comb) (Frith, 1985). Orthographic skills typically appear in children by seven years of age (Snowling \& Frith, 1981).

These developmental stages are essential in reading, and much research has suggested causal relationships between phonological awareness at preschool age and reading ability at early school age (Goswami \& Bryant, 1990; Muter et al., 1998; Rack et al., 1994; Wagner \& Torgesen, 1987). For example, Liberman, et al. (1974) tested the phonological segmentation ability of children aged four, five and six years by tapping syllables of a word, and found that there was a correlation between tapping scores and reading at early school age. In addition, a longitudinal study with 368 preschool children conducted by Bradley and Bryant (1983) provided strong evidence that those preschool children who were good in sound categorization (e.g., hill, pig, pin/cot, pot, hat/pin, win, sit) became good readers at school. The fact that phonological awareness as a good predictor of reading ability in Grade 1 has been demonstrated using a variety of 
phonological awareness tasks, such as rhyme supply/choice, deletion, alliteration, same/different initial/final consonant, and substitute consonant tasks (Muter et al., 1998; Stanovich, Cunningham, \& Cramer, 1984).

A study investigating the sensitivity of preschool children to phonemic cues (Rack et al., 1994) added to the evidence for causal relationships between early phonological awareness and reading. In the study by Rack, et al. they controlled phonemic cues in the initial (e.g., dgr for tiger) and middle (e.g., kdn- cotton) position of a word in minimal pairs. Minimal pairs are those in which two different word forms are identical in every aspect except for one sound segment that occurs in the same place in the string (e.g., pea-sea, pat-put, fine-vine, chunk-junk). The children's task was to learn to associate three- or four-letter cues (e.g., /hzpl/ for hospital, $/ \mathrm{kdn} /$ for cotton) with a spoken word, and to read a word (e.g., hospital, cotton) that they had never learned. The results showed that five-year-old preschool children could make use of phonemic cues for reading a word. Rack, et al. concluded that preschool children are sensitive enough to extract letter-sound information from phonemic cues in order to read words. Thus, ample research has shown that there are causal relationships between early phonological awareness and later reading ability.

Given the evidence that phonological awareness plays a role in learning to read, it is natural to assume that a lack of phonological awareness may block children from developing reading skills, resulting in reading difficulty. In fact, poor phonological awareness in dyslexic children is very evident in the literature (Ellis, 1991; Frith, 1981; Goswami \& Bryant, 1990; Lundberg, 1989; Snowling, 1995). That is, DD children typically perform poorly in segmentation tasks (Johnston, Anderson, \& Duncan, 1991; 
Liberman et al., 1974; Snowling, 1981), and rhyming judgment tasks, when required to recognize the similar or different sounds in a familiar words list (e.g., sun, see, sock/weed, need, deed) (Beech \& Harding, 1984; Bradley \& Bryant, 1978; Holligan \& Johnston, 1988). Snowling (1981) tested the segmentation ability of 20 dyslexic children ranging from nine to 17 years of age. In the test, the children were asked to read aloud one and two syllable real and nonsense words (e.g., wut, steg, clest, tegworp, brigbert). The findings showed that the dyslexic children had difficulty not only reading twosyllable nonwords but were also more affected by the number of consonant clusters in the two-syllable versions. The same author (Snowling, 1981) also tested a different dyslexic group consisting of 13 children (ranging from eight to 15 years of age) with a mean age of 12 years in repeating two-syllable up to four-syllable real words (e.g., enter, visitor, magnificent) and nonsense words (e.g., emper, fizidor, bagmivishent). The findings showed that the DD group performed worse, with increases of the number of consonants of the nonsense words. These studies demonstrated that phonological complexity affects reading in dyslexic children, suggesting that they have trouble segmenting a word into phonemes. As Wagner and Torgesen (1987) also emphasized the importance of phonological awareness as a prerequisite skill for reading, a lack of phonological awareness may be critical for reading difficulty in dyslexic children.

In summary, the first stage of phonological processing is encoding, in which phonological awareness is important. Numerous studies have suggested that at a preschool stage, phonological awareness is essential to becoming a fluent reader at school. Research suggests that DD children are less sensitive to phonology than normal children, and this may cause reading difficulty in DD children. 
The next section will discuss the decoding stage, which is the second component of phonological processing. An important feature of the decoding stage is access to mental phonological representation. At the beginning of the reading stage, most words are novel for young children. Children can read novel words depending on sight vocabulary, but it is limited in terms of expanding vocabulary. To read a large number of novel words, young children need to learn grapheme-phoneme conversion rules that enable them to access the mental phonological representation of a printed word (phonological recoding). The deficits of DD children in phonological recoding have been illustrated within a dual route model (Baron, 1979; Coltheart, Curtis, Atkins, \& Haller, 1993; Coltheart, Davelaar, Jonasson, \& Besner, 1977).

\section{2 Phonological Recoding and Developmental Dyslexia}

A substantial body of research supports the idea of the dual route model, in which there are at least two routes to achieving mental lexical access (Baron, 1979; Coltheart et al., 1993; Coltheart et al., 1977). The first route has been called the phonological route or phonological recoding. Phonological recoding refers to recoding written symbols into some kind of sound based representation, and then utilizing that representation to access the mental lexicon (Wagner \& Torgesen, 1987). The phonological route is especially important for beginning readers, because it appears that facility in phonological recoding in lexical access is a determinant of early reading success (Baron, 1979; Doctor \& Coltheart, 1980; Ehri \& Wilce, 1979; Stanovich, 1982). The phonological route supposedly better processes visually unfamiliar regular words (i.e., low frequency words) and nonwords according to GPC rules. Since by definition there is no lexical 
representation for nonwords in the mental lexicon, only the phonological route can provide the pronunciation of nonwords by GPC rules. The second route refers to a direct or lexical route. The lexical route is visually mediated to lexical access; i.e., written symbols and sounds pair up to access the mental lexicon. Because of the nature of the lexical route, readers who use this route can quickly process visually familiar words (e.g., cat, dog, book), high-frequency words (e.g., globe, frame, queen, telephone), and irregular words that do not follow GPC rules (e.g., have, yacht, pint).

According to Frith's stage model (1985) discussed in the previous section, preschool children are at the alphabetic stage and have rudimentary phonological awareness. These rudimentary phonological skills need to be refined to learn GPC rules at early school age. It is very important for young children to learn these rules, because they allow them to read novel words, which they encounter frequently during the early reading stage. Since DD children are less sensitive to phonological information, it is reasonable to assume that they may have increased difficulty in learning GPC rules.

A cross-modality study was conducted by Snowling (1980) on 18 dyslexic children (mean age of 12 years). The findings showed that the DD children had difficulty only when they translated from visual to auditory information, but not with other translations, i.e., visual-visual, auditory-auditory, and auditory-visual. In this study, the dyslexic children were required to answer to a series of four-letter pronounceable nonwords (e.g., sond-snod, dron-dorn) by a 'same' or 'different' response in four conditions: Auditory presentation-Auditory recognition, Visual presentation-Visual recognition, Auditory presentation-Visual recognition, and Visual presentation-Auditory recognition. The DD children performed as well as the control group in all conditions 
except the Visual-Auditory condition. Snowling argued that the Visual-Auditory condition was the most analogous to reading. She concluded that the DD children had specific deficits in converting printed words to phonological codes by graphemephoneme convergent rules.

Deficits in the use of GPC rules are typically demonstrated in reading nonwords (Baddeley, Ellis, Miles, \& Lewis, 1982; Kochnower, Richardson, Dibenedetto, 1983;

Olson, Kliegel, Davidson, \& Folts, 1985; Siegel, \& Ryan, 1988; Snowling, 1980, 1981). Baddeley, et al. (1982) studied the ability of nonword reading in DD children compared with the reading-level (age)-matched (RA) controls of 10-year-olds in real and nonword reading tasks. In their study, the RA comparison was used in order to control for different reading abilities in the experimental groups, which might affect experimental task performance. That is, DD children by definition show much poorer reading performance than a chronological age-matched (CA) group, and thus, it is not clear if poor performance in experimental tasks, such as a nonword reading task, is related to developmental delay or to other cognitive disabilities. Comparing an RA group can eliminate this possibility, although Baddeley, et al. admitted that DD children could gain additional reading strategies because they are older than their RA control group, and this may affect experimental tasks. In their study, nonwords were made up by changing a single letter of a real word (e.g., cake-dake). The results showed that DD children were worse in nonword but not in real word reading than the RA group. In another study (Frith \& Snowling, 1983), DD children (mean age of 10-12 years) were compared with an RA group (mean age of eight to 10 years) in reading regular (e.g., coffee, spade), irregular words (e.g., biscuit, laugh) and nonwords (e.g., slosbon, molsmit). Frith and Snowling 
found that the DD children read the real words equally well to their RA group; however, their performance in reading nonwords was much worse than the RA group.

Olson, et al. (1985) also compared DD children (mean age of 15.03 years) with RA children (mean age of 10.2 years) in a phonological choice task. In this task, the children were asked to choose a nonword that was rhymed with a real word (e.g., cakedake). The DD children performed significantly worse than their counterparts in this task. When the DD children were asked, however, to correctly identify a word from a homophonic misspelled word (e.g., street, streat), they did not differ from their counterparts, indicating that the lexical knowledge of the DD children in this sample was unimpaired. Other studies have provided the same line of findings (Kochnower et al., 1983; Siegel \& Ryan, 1988). A review by Rack, et al. (1992) compiled findings from ten papers, which involved a total of 428 dyslexics and a similar number of normal readers, and concluded that dyslexic children are worse than RA normal readers on measures of phonological reading skills. These results suggest that DD children have deficits in phonological skills.

Despite the positive results described previously, there are some discrepancies in the literature. Beech and Harding (1984) found no difference between DD children and an RA group in terms of reading two-syllable nonwords (e.g., dention), regular words (e.g., suffer) and irregular words (e.g., sugar). These results led the authors to conclude that DD children are better characterized as developmentally delayed. This developmental delay hypothesis states that DD children take normal reading stages as Frith's stage model (1985), but move through the reading stages more slowly than normal children. This hypothesis was supported by other studies (Bruck, 1988; Treiman, \& 
Hirsh-Pasek, 1985). Furthermore, Vellutino and Scanlon (1987) added strong evidence against the phonological deficit theory. In their study, 75 DD children (mean age of 12.0 years) and 75 RA normal readers (mean age of 7.09 years) were compared in reading 20 real words and 20 nonwords created by changing one letter in each of 20 different real words. The majority of real and nonwords were regular (e.g., lamp, wash, dronk, rosk). The results showed that the DD children did not differ from their RA group, indicating that there is no specific nonword deficit in DD children. Rack, et al. (1992) pointed out, however, that the testing word list used by Vellutino and Scanlon should have included irregular words, as the Beech and Harding study did, because comparing the performance between reading nonwords and irregular words typically demonstrates dyslexic phonological deficits.

These inconsistent results raised a debate as to whether reading difficulty in DD children represents a specific phonological deficit or a normal developmental delay. The positive findings suggest that the reading difficulty of DD children is specific to the area of phonological skills, whereas the negative results led some researchers to conclude that dyslexia is better conceptualized as a developmental delay. One way to answer this question is to compare DD children with CA readers, RA readers, and CA "poor" (CAP) readers. The later group is a chronological age-matched group with the DD group. However, their poor reading ability may be caused by a variety of reasons, such as low IQ or a lack of opportunity (Gough \& Tummer, 1986). Gough and Tummer hypothesized that if DD children perform worse than CA readers, but the same as both RA readers and CAP readers, then their reading difficulty must be due to a developmental delay. However, if DD children show worse performance than RA readers and show different 
patterns of reading deficits than CAP readers, this would be conclusive evidence that the reading deficits of DD children are specific and do not represent a developmental delay.

Swan and Goswami (1997) compared DD children (mean age of 12 years) with CA, RA (mean age of 9.8 years) and CAP groups in picture/word naming and object naming tasks, in which the word frequency (high and low) and the length (three to five syllables) were manipulated. In the picture/word naming task, the children were asked to name a picture. Overall, the CA group showed the best performance among the groups in all conditions. The second best was the RA group. The DD children showed equally good performance to the RA group in the short, high frequency word condition (e.g., dust, clock, flag), but exhibited worse performance than the RA and CAP groups in the long, low-frequency word condition (e.g., propeller, binoculars). Moreover, the CAP group demonstrated poor performance for all types of words, whereas the DD children showed poor performance for only long and low frequency words. Swan and Goswami interpreted this finding as DD children having difficulty in specifying and retrieving the phonology of names from the mental lexicon.

In the same study, the children were also asked to point to a picture while listening to a word spoken by the examiner (i.e., object-naming task). Among the 40 target pictures, the target words were those for which the DD children could not identify the names in the previous picture/word-naming task, i.e., they failed to name the words from memory. The results showed that the DD children obtained significantly higher scores than the other control groups in naming the target words. Surprisingly, they showed good performance in the target words that they had failed to name in the previous picture/word naming tasks. In contrast, the CAP group showed the worst 
performance among the groups in all types of words. These findings confirmed that DD children have a specific retrieval problem from the mental lexicon.

Error analysis revealed that the DD children made more phonological errors (e.g., $\operatorname{cog}$ for clog) than errors in semantically related words (e.g., elevator for escalator) or perceptually related words (e.g., stem for wick) compared to other groups who made errors equally in any type of words. Swan and Goswami (1997) concluded that the difficulty for DD children is to retrieve the phonological codes of pictures from the mental lexicon; in contrast, the CAP group has the problem of impoverished vocabulary in the mental lexicon.

In summary, phonological recoding is an important mental process in reading, because it allows readers access to word of sounds in the mental lexicon and to read novel and unfamiliar words. This processing can be achieved through GPC rules. The dyslexia literature suggests that the main deficit in DD children lies in their difficulty in learning these rules and that their lack of phonological skills causes DD.

While encoding, access to the phonological mental representation and retrieving sounds of a word, the information contained in a word needs to be maintained in working memory (WM). WM refers to "temporary storage and manipulation of the information necessary for such complex cognitive tasks as language, learning and reasoning" (Baddeley, 1992). Previous research suggests that WM also plays an essential role in the acquisition of reading skills (Brady, 1991; Gathercole \& Baddeley, 1989; 1990; Jorm, 1983; Macaruso, Locke, Smith, \& Powers, 1996; Mann \& Liberman, 1984; Torgesen, 1988; Wagner \& Torgesen, 1987). In the early acquisition of reading skills, it is very important to learn how to segment phonemes, map them onto sounds, and blend together 
isolated phonemes to make words. It is necessary to transform spoken sounds into mental phonological representations; otherwise the information quickly fades away (Cowan, 1995). These tasks require WM. Thus, phonological coding in WM is closely connected to acquiring reading skills (Oakhill \& Kyle, 2000). Previous research has shown that DD children demonstrate poor performance in recall memory tasks (Liberman et al., 1977; Mann \& Liberman, 1984; Mann, Liberman, \& Shankweiler, 1980). The next section will discuss the connection between phonological WM and reading disability.

\section{3 Working Memory and Developmental Dyslexia}

The term working memory (WM) was originally conceptualized by Baddeley and Hitch (1974) to emphasize the active component of the storage role of short-term memory (STM). Baddeley (1986) later elaborated the concept of WM. He argued that WM has a limited capacity for the temporary holding and manipulating of information during the performance of a range of cognitive tasks such as comprehension, learning and reasoning. WM has three components: the central executive, the visuo-spatial scratch pad, and the articulatory loop. The central executive, which functions as the control center of the system, is assumed to select and operate various processes. The other two components are subsidiary slave systems. The visuo-spatial scratch pad deals with constructing and manipulating visual imagery. The articulatory loop is specialized for the temporary storage of phonological/verbal material and also for the articulatory rehearsal process referred to as working memory. Verbal information can be registered either by auditory or subvocal rehearsal. Thus, phonological codes play an important role in storing verbal information in WM. 
Many studies have suggested that phonological WM is essential in reading (Corkin, 1974; Gathercole \& Baddeley, 1989; Liberman et al., 1977; Mann \& Liberman, 1984; Shankweiler, Liberman, Mark, Fowler, \& Fischer, 1979). For example, Katz, Shankweiler, and Liberman (1981) demonstrated that good and poor (normal) beginning readers did not differ in a memory task involving ordering stimuli that are difficult to label; however, good readers were significantly better than poor readers in ordering stimuli that were amenable to labeling. The results indicate that good beginning readers can make good use of phonological WM.

Furthermore, Mann and Liberman (1984) investigated the interrelationships between phonological WM and reading, specifically, the association between the memory spans of preschool children and the reading of first grade children. They tested 62 normal children twice, in kindergarten and the first grade, using a syllable counting test, a word-string memory test with phonetically confusable (rhymed) and nonconfusable (non-rhymed) words. The scores from two standardized reading tests (the Word Attack and Word Recognition sub-tests of the Woodcock Reading Mastery Test; Woodcock, 1987) were used to group the children into three reading groups: good, average and poor (normal) readers. The results showed that the good readers could count syllables significantly better (85\%) than the other reading groups (56\% for average and $17 \%$ for poor), and that the number of errors in the word-string memory test was inversely related to reading ability. In addition, the phonetically non-confusable items dissociated the three reading groups more than the phonetically confusable items. This finding was interpreted as an indication that the good readers were more efficient in the use of phonological codes in memory tests, and that this resulted in their being more 
penalized by the presence of phonetically related items than the other reading groups. More interestingly, the same results carried on to the first grade. That is, in both syllable counting and WM tests, those who did well in kindergarten became good readers in the first grade, and those who were average in kindergarten became average readers at the first grade, and so forth. Therefore, Mann and Liberman concluded that both phonological awareness and WM are critical elements in reading and can predict future reading ability at early school age.

If both phonological awareness and WM are essential in developing reading ability, the natural assumption is that DD children might have poor phonological WM, since poor phonological awareness is documented in DD children. In fact, previous studies with DD children suggest that they have poor phonological WM (Breznitz, 1997; Gathercole \& Baddely, 1990; Kramer et al., 2000; Macaruso et al., 1996; Palmer, 2000). For example, Macaruso et al. (1995) tested the verbal WM of 26 DD children ranging from 10 to 19 years and 26 controls raging from nine to 20 years. The participants were asked to touch a series of drawings in the order in which they were originally presented. The stimuli consisted of three sets of six line drawings: the short-rhyme (e.g., bees, cheese, trees), short-non-rhyme (e.g., bell, chair, dog), and long non-rhyme set (e.g., banana, butterfly). The results showed that overall the DD children performed more poorly than the controls in the serial position task, suggesting that the DD children have a WM impairment. The serial position curve (Wickens, 1972) refers to the probability of correct recalls of items presented in serial positions and the classic curve is bow-shaped with high probabilities for the first few (primacy effect) and last few items (recency effect). The presence of the rhyme effect at the primacy but not the recency effect of the 
serial positions also indicated that the children could make use of phonological codes in their limited WM. Contrary to the DD children, the results revealed that the good readers showed a typical serial position curve and a rhyme effect. This suggests that the good readers can make better use of phonological codes in WM than the DD children. These findings are consistent with the view that good readers can utilize the articulatory loop in WM (Liberman et al., 1977, Katz et al., 1981). The finding of a rhyme effect at the early serial position in DD children, however, supports the view that their memory deficit is due to WM but not to long-term memory (Gathercole \& Baddeley, 1990; Kramer et al., 1999). Macaruso, et al. explained that the reasons DD children did not show the rhyme effect at later serial positions as the good readers did was because they switched from phonological coding to other coding strategies, such as visual coding in WM. They suggested that DD children make use of less precise phonological codes in WM than good readers do, as reflected in their reading difficulties.

To summarize, WM is believed to be essential for reading. It is a good predictor, as is phonological awareness, of reading performance at early school age. DD children have been shown to have limitations in verbal WM capacity, which might contribute to their reading difficulty in various ways.

It is well documented that DD children have poor phonological awareness and decoding problems. These problems, however, are not uniformly demonstrated in DD children. Previous studies (Howes, Bigler, Lawson, \& Burlingame, 1999; Boder, 1973; Castles \& Coltheart, 1993; Hendriks \& Kolk, 1997) have suggested that there may possibly be sub-types of dyslexia. The next section will explore sub-types of DD. 


\subsection{Sub-types of Developmental Dyslexia}

Since the early 1970s, researchers have speculated that there are sub-types of DD. Boder $(1971,1973)$ classified DD into three sub-types based on characteristics of reading difficulty: (1) dyseidesia, (2) dysphonesia, and (3) dysphoneidesia, based on clinical observation of reading problems. The first sub-type, dyseidesia, refers to a deficit in perceiving whole words as visual gestalts and matching these words with auditory sounds. Thus, dyseidetic children have fewer problems in reading regular words (e.g., stop, teach, cake), but more problems in reading irregular words (e.g., yacht, pint, have). The second sub-type, dysphonesia, refers to a difficulty in reading words using grapheme-phoneme rules. The dysphonetics' mistakes mainly lie in regular words. This type is considered to be the most common among DD (i.e., 55-70\%) (Flynn \& Boder, 1991; Flynn \& Deering, 1989). The third sub-type, dysphoneidesia, is defined as a combination of both types of deficits and is generally considered to be a more severe form of DD than the other sub-types.

Other researchers (Castles \& Coltheart, 1993; Coltheart et al., 1983; Ellis, 1994; Temple, 1984) classified DD into two sub-types; phonological DD and surface DD based on similarity to alexia, a term which refers to reading difficulty caused by brain damage (Patterson, Marshall, \& Coltheart, 1985). Phonological DD refers to those who cannot read words by GPC rules; as a result, typical deficits are manifested in reading nonwords. Surface DD refers to those who show impairment in reading irregular words but have relatively normal ability in reading regular or nonwords. Although different researchers have identified sub-types of DD, they all identify similar groups as comprising these sub- 
types, i.e., as problems having either with phonological processing or problems with visual processing (such as whole word processing).

The dual-route model for word recognition as discussed previously fits well to the classification of DD. Researchers who advocate the existence of sub-types of DD have proposed that the two types of DD represent deficiencies in either the lexical or phonological route (Boder, 1973; Castles \& Coltheart, 1993; Coltheart et al., 1983; Ellis, 1994). For example, in a study of Coltheart, et al. (1983), a surface DD girl (17 year-old), whose reading age was 10 years with an IQ of 100 , demonstrated better performance in reading regular words than irregular words and made frequent regularization errors in reading irregular words. Another surface dyslexic case reported by Temple (1984) was a 13-year-old boy (NG). NG demonstrated better performance in reading regular words ( $48 / 50$ words) than irregular words ( $28 / 50$ words), where the difference was statistically significant. NG also showed good performance in reading regular words (i.e., 16/20 regular words: preferential, procrastinate, statistics) from the 14 and 15-year-old level of the Schonell Test (Schonell \& Goodacre, 1971). These case studies illustrate that some DD children have more impairment in visual processing than phonological processing.

One of the criticisms of the concept of surface dyslexia is that children with this condition might use different reading strategies which are not typically controlled in the case studies (Snowling, 1987; Wilding, 1989). For example, a DD child might switch from the lexical to the phonological route and vice versa in reading to compensate for their deficiency (Hendriks \& Kolk, 1997). Against this criticism, Castles and Coltheart (1993) investigated a large sample of dyslexic children (56 dyslexic children and 56 controls). Their findings indicated that there were some DD children who showed 
impairment in reading irregular words but whose reading of nonwords was within the normal range and vice versa. A recent study (Sprenger-Charolles, Cole, Lacert, \& Serniclaes, 2000) attempted to replicate the Castles and Coltheart study in the French language. They failed to find any surface DD children in their sample. SprengerCharolles, et al. argued that as previous studies have demonstrated (Ehri, 1998; Perfetti, 1992; Share, 1995), the acquisition of phonological skills is a prerequisite to developing the orthographic mental lexicon. They concluded that the core deficit in DD is phonological processing and discarded the idea of sub-types of DD. There is, however, still considerable controversy surrounding the issue.

\subsection{Summary}

The phonological deficit theory assumes that DD is primarily a linguistic disorder. This linguistic disorder is apparent in both poor phonological awareness and decoding problems. Poor phonological awareness is manifested in sound categorization or phonemic segmentation tasks. Since phonological awareness is essential in learning to read, especially at preschool age, poor phonological awareness in DD children interferes with the development of their reading skills. The decoding problem is best demonstrated by nonword reading, because DD children have difficulty in learning the grapheme-tophoneme conversion rules required to read novel words and nonwords. In naming tasks, DD children have difficulty in naming unfamiliar or long words, whereas slow learners or low IQ readers have difficulty in remembering all types of words, suggesting that DD children have retrieval problems and slow learners or low IQ readers have poor lexical knowledge in the memory. There is a correlation between verbal WM at preschool age 
and reading ability at early school age. Dyslexic children performed more poorly in a WM task than the control group, suggesting that their WM is limited.

There is controversy over the existence of sub-types of developmental dyslexia. One camp claims that the core deficit in DD is in phonological processing, while the counterpart claims that there are at least two types of developmental dyslexia, which demonstrate either a deficit in phonological processing or visual processing respectively.

\subsection{Discussion}

In the DD literature, the importance of phonological awareness, decoding skills and verbal WM is clear (Ehri, 1991; Ellis, 1991; Foorman \& Liberman, 1989; Goswami \& Bryant, 1990; Gough, 1996; Hulme \& Snowling, 1992; Muter et al., 1997; Snowling, 1995; Wagner \& Torgesen, 1987). The strength of the phonological deficit theory is grounded in the wide body of reading literature. Furthermore, numerous studies with DD children have convincingly provided evidence that DD children have poor phonological awareness (Ellis, 1991; Frith, 1981; Goswami \& Bryant, 1990; Lundberg, 1989;

Snowling, 1995), poor decoding skills (Frith \& Snowling, 1983; Kochnower et al., 1983;

Rack et al., 1992; Siegel \& Ryan, 1988; Swan \& Goswami, 1997) and poor verbal WM (Cathercole \& Baddeley, 1990; Liberman et al., 1977; Macaruso et a., 1995). It is now widely accepted that DD children have phonological deficits (e.g., Rack et a., 1992; Snowling, 1995, 2000).

There are several weaknesses in this theory, however. First, the phonological deficit theory does not discuss the origin of the linguistic disorder. Second, the theory focuses on two elements of phonological processing: phonological awareness and phonological recoding, but not on the third element, working memory (WM). Since the 
importance of WM in reading at an early age is evident in the reading literature, the theory should include WM. Third, difficulty in reading fluency must be accounted for by the theory, because previous studies with DD children (Denckla \& Rudel, 1976; Fawcett \& Nicolson, 1994; Mcbride-Chang, Manis, 1996; Van der Leij, \& Van Daal, 1999; Wimmer, Mayringer, \& Landerl, 1998) found that DD children demonstrated slow naming speed for well learned objects. This suggests that they may have deficits in the automatic retrieval processing of each name from the mental lexicon. The theory as currently stated does not include this element. Fourth, the phonological deficit theory dismisses the existence of sub-types of dyslexia. However, dyslexia research conducted using small samples (e.g., 10 from Snowling, 1981; 9 from Frith, 1985; 15 from Baddeley et al., 1988) may not be able to delineate a true picture of dyslexia given the diverse individual differences among DD children (Castle, \& Coltheart, 1994; Ellis, 1994). Studies with large samples need to be done in English and other languages, especially given that the French language study (Sprenger-Charolle et al., 2000) did not find sub-types of dyslexia. Fifth, the phonological deficit theory does not explain other characteristics of dyslexic children, such as visual problems, poor bimanual coordination or anatomical features. Finally, this theory was developed to account for difficulty in learning to read one language, i.e., English. The theory needs to examine visually based (logographic) languages, such as Japanese, as to whether a phonological processing deficit in dyslexia is valid across languages.

The next chapter will discuss the left hemisphere dysfunction theory focusing on structural and functional abnormalities in the DD brain. 


\section{Chapter 3: The Left Hemisphere Dysfunction Theory}

The hypothesis that abnormal development of the cerebral hemispheres might cause DD has been put forward since the first case was reported (Hinshelwood, 1917; Orton, 1937; Bakker, 1979,1992). No concrete evidence, however, supported the hypothesis until autopsy studies (Drake, 1968; Galaburda, \& Kemper, 1979; Hier, LeMay, Rosenberger, \& Perlo, 1978) found that many adult dyslexic brains have neuronal abnormalities in the left hemisphere $(\mathrm{LH})$, and in many cases the pattern of cerebral asymmetry in the language area was reversed from that of normal brains. Furthermore, recent functional neuroimaging studies have demonstrated functional disruption in the left hemisphere. The left hemisphere dysfunction theory holds that abnormalities in brain organization confined to the LH cause dyslexia. This chapter will examine the relationships between DD and abnormalities in the LH. The chapter will be divided into five sections: (1) cerebral specialization, (2) cerebral asymmetry, (3) developmental dyslexic brains, (4) summary, and (5) discussion.

\subsection{Cerebral Specialization}

Hemispheric specialization refers to each cerebral hemisphere being specialized for different cognitive abilities (Hellige, 1983; Zaidel, 1983). This view was developed from the concept of cerebral dominance (Jackson, 1868). The cerebral dominance view holds that only one hemisphere dominates or leads mental functions. The discovery by Broca (1865) and Wernicke (1874) that damage in the LH but not in the right hemisphere $(\mathrm{RH})$ leads to language disorders contributed to this view of cerebral dominance. At the 
time, language was considered to be the central cognitive function with the LH having sole responsibility for language functions and the RH being mute (Springer \& Deutsch, 1989). This view prevailed in the scientific world until Roger Sperry and associates (Sperry et al., 1975) tested the competence of each hemisphere with commissurotomized patients. Commissurotomized patients are those whose callosal commissures (fiber tracts connecting both cerebral hemispheres) have been surgically sectioned to prevent the spreading of epileptic seizures to the opposite hemisphere.

A series of studies with commissurotomized patients (Bogen \& Gazzaniga, 1965; Levy \& Trevarthen, 1976; Levy, Trevarthen, \& Sperry, 1972) revealed that the RH has different cognitive functions from the $\mathrm{LH}$, such as visuo-spatial abilities. The studies used special techniques, such as the tachistoscopic technique, which allows the measurement of hemispheric differences in the visual modality. In this technique, a visual stimulus is presented in either the left visual field (LVF) or right visual field (RVF). The critical aspect of the presentation is that a stimulus presented in the LVF projects its image in the RH, and a stimulus presented in the RVF projects its image in the LH when a subject focuses on a centered fixated point. After the initial projection in each hemisphere, the information crosses the corpus callosum (CC) to the opposite hemisphere (see Appendix A). When the CC is sectioned in commissurotomized patients, information is confined to one hemisphere. Studies with commissurotomized patients demonstrated that these patients could identify spatial objects such as faces or pictures better when they were presented in the LVF/RH than the RVF/LH. In contrast, verbal stimuli were identified better when they were presented in the RVF/LH than the LVF/RH. These findings suggest that the $\mathrm{RH}$ is specialized in spatial processing and 
facial recognition and the $L H$ is predominantly specialized in verbal processing, especially phonological processing and speech production (Sperry et al., 1969; Zaidel, 1977).

The dominant role of the left hemisphere in processing language was further supported by alexia research (Ellis, Flude, \& Young, 1987; Friedman, Ween, \& Albert, 1993; Patterson \& Kay, 1982; Patterson et al., 1985; Shallice \& Warrington, 1980). Alexia refers to a disorder of reading and writing resulting from various types of brain injuries in the posterior brain regions, such as stroke, brain hemorrhage or accidents (Shallice \& Warrington, 1980). The findings suggested that the left angular gyrus, including Broca's area and Wernicke's area, is important for reading processing (Figure $3.1)$.

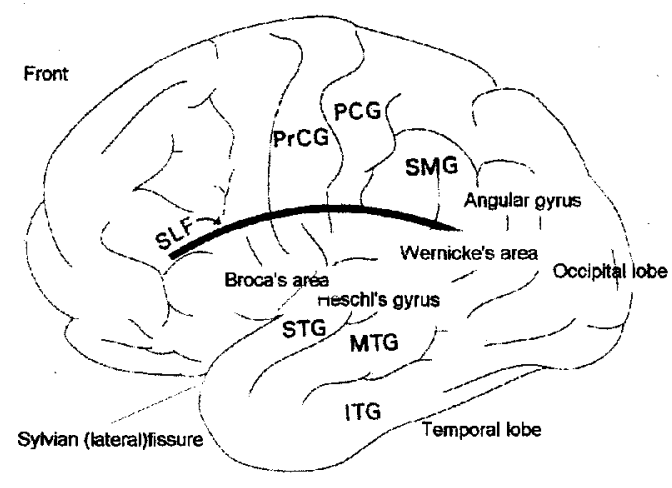

Figure 3.1. Reading pathways based on alexia research.

$\mathrm{PrCg}=$ precentral gyrus; $\mathrm{PCC}=$ postcentral gyrus; $\mathrm{SMG}=$ supramarginal gyrus; $\mathrm{MTG}=$ medial temporal gyrus; $\mathrm{ITG}$ = inferior temporal gyrus; $\mathrm{OL}$ = occipital lobe; $\mathrm{SLF}$ = superior longitudinal fasciculus (of which the arcuate fasciculus is a part) (DeArmond et, al., 1974).

Broca's area, located in the anterior LH, is specifically associated with speech production (Friedman et al., 1993). Typically, when right-handed patients have lesions in Broca's 
area, it causes inability to produce speech, a condition referred to as Broca's aphasia (Benson \& Geschwind, 1985). Wernicke's area, located in the left parietal temporal region, is important for comprehension. Disruption in this region causes inability to understand speech, known as Wernicke's aphasia (Hecaen \& Albert, 1978; Hier \& Mohr, 1977). Mesulam (1990) has described a large reading network including three large regions within the LH: the left anterior regions including Broca's area being closely related automatically to motor output system, the posterior left temporal-parietal regions (dorsal temporal lobe) related to phonological processing and the posterior left occipitaltemporal regions (ventral temporal lobe) including Wernicke's area related to semanticlexical processing. The angular gyrus located in the posterior portion of the parietal lobe receives all inputs associated with reading from dorsal and ventral temporal regions and connects these inputs with Broca's area (Friedman et al., 1993). Thus, the angular gyrus (Figure 3.1) appears to act as a relay station between the posterior and the anterior regions when the brain is processing printed information. When brain damage in this region spares the angular gyrus, alexia occurs without agraphia (disability of writing). This is called pure alexia (ability to write but inability to read one's own or other's writing). When the angular gyrus is damaged in addition to the other three regions, alexia occurs with agraphia (inability to both write and read) (Friedman et al., 1993). When the temporal parietal region is disrupted, phonological reading is interrupted (phonological route) but whole word reading remains intact, in which case the patient is described as having phonological alexia (Funnel, 1983). When the occipital-temporal region is disrupted, a patient' s reading becomes letter-by-letter because of the loss of the ability to read the whole word (direct orthography-to-phonology route). In this case, the patient is 
described as having surface alexia (Patterson \& Kay, 1982; Patterson et al., 1985). When both routes are blocked, semantic paralexia occurs, in which reading errors in meaning are demonstrated. That is, reading errors are seen in resembled words (pretty -> beautiful), synonyms (attorney -> lawyer), superordinate words (cat -> animal), or associated words (sleep $->$ dream). This is also called deep alexia (Friedman et al., 1993). Functional neuroimaging studies have suggested that Broca's area is also closely related to phonological processing at the point where it becomes more complex processing (Brunswick, McCrory, Price et al., 1999). Thus, ample evidence suggests that the left hemisphere is important for reading processing. Interestingly, however, a cross-cultural study of alexia using the Japanese language revealed that initial feature analysis may be achieved in the right hemisphere because pure alexia was always accompanied by hemianopia in the RH (blindness in one half of the visual field) (Sugishita, Otomo, Kabe, \& Yunoki, 1992).

In addition, in the Japanese language, the distinction between surface and phonological alexia is much clearer than in the English language. In Japanese, two distinct writing systems, Kanji (ideograms) and Kana (phonograms), are typically used in combination in sentences. The details of these two writing systems are quite intricate and beyond the scope of this chapter. However, an interesting fact is that Japanese adult patients with surface alexia show impairment mainly in Kanji while maintaining Kana reading intact (Sasanuma, 1974,1985). In contrast, Japanese phonological alexia patients show impairment in Kana while maintaining Kanji knowledge (Sugishita et al., 1992). This confirms that there are two major networks in the brain for reading. 
Since the patterns of reading impairment in DD appear to be similar to those observed in alexia patients, the term 'developmental dyslexia' was derived from this observation. Although the two clinical presentations look alike in some respects, alexia patients were fluent readers before their brain damage; in that respect they are a totally different population than developmental dyslexics.

To summarize, the concept of cerebral specialization emphasizes that each hemisphere is specialized for different cognitive abilities, and denies the idea that only the LH plays a key role and the RH has little role in cognitive functions. Since the data are derived from clinical populations such as commissurotomized patients who never had normal brains due to their long history of epileptic seizures, and brain damaged patients whose brain damage is usually diffused, there must be some reservation in generalizing the results. According to the concept of cerebral specialization, the LH is predominantly responsible for language, especially for phonological processing (Sperry et al., 1969; Zaidel, 1979).

Since poor phonological processing is a distinguishing characteristic of DD children, it logically follows that some abnormalities in the LH might cause their reading disability. Furthermore, it has been assumed that the neuronal organization in the language area might be abnormal in DD brains. Before proceeding to further discussion, it is necessary to first consider normal brain organization, which is characterized by leftward asymmetry in the brain. 


\subsection{Cerebral Asymmetry}

Despite appearances, the human cerebral cortex is not completely symmetrically formed, that is, homologous regions may differ in size in the two hemispheres. For example, for right-handers, the right frontal lobe is typically wider than the left and the left posterior occipital lobe is typically wider than the right. This difference is smaller for left-handers than right-handers, and some left-handers have a symmetrical or reversed asymmetrical (i.e., rightward asymmetry) organization of the cerebral hemispheres (Galaburda, LeMay, Kemper, \& Geschwind, 1978) (Figure 3.2, CT scan, see Appendix B). In the normal (non-dyslexic) brain, this asymmetrical organization is typically observed in the language area, the Planum Temporale (PT), which is in the cortical area lying between Heschl's gyrus and the posterior margin of the Sylvian fossa (see Figure 3.1). An autopsy study of 100 normal brains (Geschwind \& Levitsky, 1968) investigated the relative difference in size between the left and right PT (Figure 3.3).

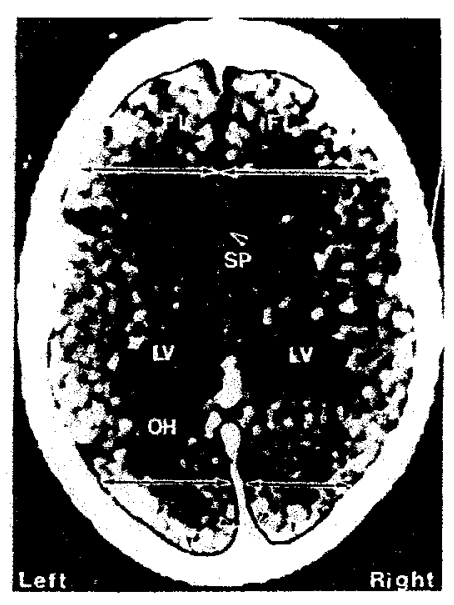

Figure 3.2. Computer Axial Tomogram (CT) of a brain shows the usual pattern of hemispheric asymmetry. Note the wider right frontal lobe (FL, upper arrows), and the more prominent left occipital horn (OH) of the lateral ventricles (LV). The septum pellucidum (SP) denotes the midline. Note also the left occipital pole distorting into the right side, a common situation (lower arrowhead) (Galaburda et al., 1978). 

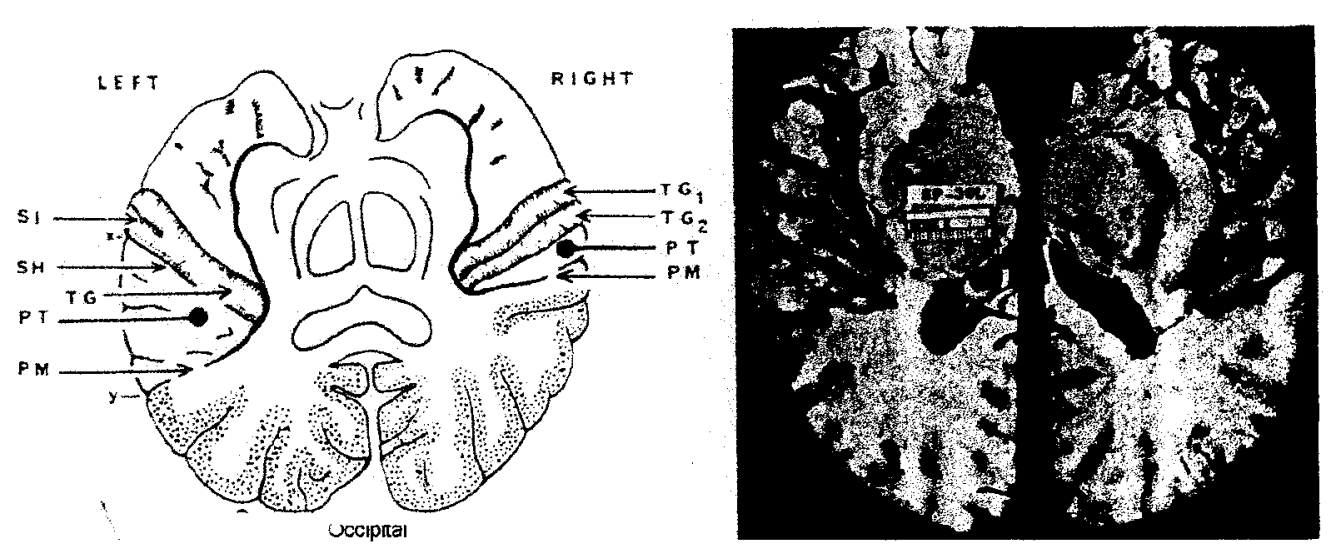

Figure 3.3. Anatomical landmarks and typical left-right differences.

(Left) The illustration of upper surfaces of human temporal lobes exposed by a cut on each side in the plane of the Sylvian fissure. (Right) Upper surfaces of temporal lobes exhibits typical right-left differences. $\mathrm{PM}=$ posterior margin; $\mathrm{PT}=$-planum temporale; $\mathrm{SH}=$ Heschl's gyrus $(\mathrm{PT}) ; \mathrm{SI}=$ Sulcus intermedius of Beck; TG = transverse gyrus of Heschl. Upper surfaces of human temporal lobes exposed by a cut on each side in the plane of the Sylvian fissure. PT = planum temporale. Upper surfaces of temporal lobes exhibit typical right-left differences (Geschwind \& Levitsky, 1968).

Geschwind and Levitsky (1968) found that $65 \%$ of the sample had a larger left PT (leftward asymmetry), $11 \%$ had a larger right PT (rightward asymmetry), and the rest (24 \%) were symmetrical, although the handedness of the sample was unknown. A striking fact is that the leftward asymmetry brains showed a left PT on average five or more times larger than the right one. Wada and his colleagues (1975) subsequently found that this cerebral asymmetry was observed as early as week 31 of gestation, and concluded that the leftward asymmetry of the PT may be genetically programmed.

Although the precise determinants of cerebral asymmetries in developing human brains are not known (Schachter, 1993), a hypothesis was put forward to explain asymmetrical cortical development (Geschwind \& Galaburda, 1985a, 1985b, 1985c; 
Rosen, Sherman, \& Galaburda, 1993). This hypothesis claimed that leftward asymmetry is the normal brain organization for maximal functional efficiency, and the symmetrical or reversed asymmetrical (rightward) PT results from a failure in neuronal pruning (cell elimination). It was speculated that at the earliest stage of brain development, neurons are overproduced in both PT areas (large symmetrical PT), and before the end of the second month of gestation, normal leftward asymmetry emerges due to genetically programmed neuronal pruning. That is, cell death occurs more in the right PT than the left PT. It is further speculated, however, that an intrauterine factor, such as testosterone, may influence cortical development and alter generically programmed neuronal pruning; i.e., if testosterone is excessively active, it interferes with the pruning and enhances right planum growth, resulting in either symmetry or rightward larger Plana.

In summary, $65 \%$ of normal brains have leftward asymmetry PT, $11 \%$ of normal brains have rightward asymmetry PT and the rest (24\%) show symmetrical brains. It is speculated that the leftward asymmetrical brain is due to brain pruning at the early stage of brain development, and the asymmetrical and symmetrical brains result from a failure of pruning due to the influence of testosterone.

About $35 \%$ of normal brains are symmetrical or reversed asymmetrical with respect to the PT areas (Geschwind \& Levitsky, 1968). The left hemisphere dysfunction theory, however, claims that the abnormal organization of the PT areas is mainly responsible for DD. Studies using autopsy and brain scanning methods (Dalby, Elbro, \& Stodkilde-Jorgensen, 1998; Drake, 1968; Duara, Gross-Glenn, Barker et al., 1991; Galaburda \& Kemper, 1979; Galaburda et al., 1985; Hier et al., 1978; Humphreys, Kaufmann, \& Galaburda, 1990; Hynd et al., 1990; Larsen et al., 1990; Pennington, 
Filipe, Lefly et al., 1999) suggest that the DD brain appears more likely to have a symmetrical or reversed asymmetrical PT than the non-DD brain. Supporting this theory, the next section will discuss the abnormal organization of dyslexic brains.

\subsection{Developmental Dyslexic Brains}

This section will focus on two topics: (1) structural abnormalities and (2) functional abnormalities.

\subsubsection{Structural Abnormalities}

Structural abnormalities in DD brains have been observed in the form of symmetry or rightward asymmetry in the PT areas and also in the presence of deformed cells in the left PT area (Drake, 1968; Galaburda \& Kemper, 1979; Hier et al., 1978; Galaburda et al., 1985; Humphreys et al., 1990; Larsen et al., 1990). Although the Hier, et al. study using CT scan did not specifically focus on the PT areas, this study is important because it showed that ten out of 24 adult dyslexic participants (42\%) had reversed asymmetry (wider in the right) in the parieto-occipital region. The authors argued that this pattern normally occurs in only $9 \%$ of right-handers and $24 \%$ of lefthanders. Thus, the frequency was much higher than would be expected normally. They further found that the dyslexic participants who had a rightward wider parieto-occipital region showed more severely delayed speech over the developmental course and had lower verbal IQs compared to the dyslexic participants who had normal asymmetry (larger in the left) in the region. The authors suggested that symmetrical and reversed cerebral asymmetry (i.e., atypical pattern) in the parieto-occipital region might increase the risk of reading disability. They emphasized, however, that $\mathrm{DD}$ is probably not caused 
by symmetrical or reversed cerebral asymmetrical brain organization alone, but must involve other factors, such as cell malformation.

In fact, autopsy studies (Drake, 1968; Galaburda \& Kemper, 1979; Galaburda et al., 1985) found anatomical malformations in dyslexic brains. An autopsy study of a 12 year-old dyslexic boy (Drake, 968), who had been examined by a psychologist because of his reading problems prior to his accidental death, found that his brain had a much larger cerebral cortex than a normal brain as well as numerous ectopic cells (warts) in the white matter. This finding indicated that there were some anomalies in his brain. Another autopsy study (Galaburda \& Kemper, 1979) found that a 20 year-old dyslexic male had cytoarchitectonic abnormalities, primarily in the left cerebral hemisphere.

Cytoarchitectonics refers to the study of the arrangement and morphology of cells, which serve to delineate distinct regions within the brain (Galaburda et al., 1978). Before a sudden accidental death by falling, this patient showed persistent reading difficulty despite special tutoring. The autopsy showed that the PT areas were approximately symmetrical, and more strikingly, adjacent molecular layers of the abnormal gyri (the convoluted portion of the cerebral cortex) were frequently fused. More importantly, the malformation was confined to the left auditory region (inferior temporal region) around the PT area characterized by ectopias in layer 1 (the surface layer of the cerebral cortex). Since the cortical cell layers are formed from layer 6 , and layer 1 emerges at the last stage of cell development (Kelly, 1991), the presence of anomalies in layer 1 suggests that the disorder takes place in late cell migration during prenatal development. The results of these two early autopsy studies suggested that the dyslexic brain is structurally and anatomically abnormal. 
Subsequently, Galaburda, et al. (1985) found similar symmetrical PT and cortical anomalies in the autopsy examination of an additional five male dyslexic brains. Cortical anomalies were found in the inferior frontal and superior temporal regions of both hemispheres. However, these anomalies were more numerous in the LH than the RH. The anomalies were again characterized by ectopias and dysplasias (deformities of cells) in layer 1 of the cerebral cortex. The absence of normal asymmetry in the PT areas was also noticed in these dyslexic brains. Thus, anatomical evidence suggests that abnormal asymmetry or symmetry PT, cell malformation and abnormal cell migration during brain development may cause developmental dyslexia.

The early findings from autopsy and CT scanning studies (Drake, 1968; Galaburda \& Kemper, 1979; Galaburda et al., 1985; Hier et al., 1978, Humphreys et al., 1990) have been confirmed using modern brain scanning techniques (Dalby et al., 1998; Duara et al., 1991; Hynd et al., 1990; Larsen et al., 1990; Pennington et 1., 1999; Duara et al., 1991; Humphreys et al., 1990; Hynd et al., 1990; Larsen et al., 1990).

The first magnetic resonance imaging (MRI) study of developmental dyslexics (Larsen et al., 1990) (for information about MRI, see Appendix C) demonstrated that 13 out of 19 DD children in grade $8(68 \%)$ had symmetrical plana or a larger right planum, whereas only five out of 17 normal controls (29\%) had symmetrical plana or right PT. This MRI study confirmed the landmark findings of Geschwind and Levitsky (1968) that $25-30 \%$ of normal brains have symmetry or rightward asymmetry. An MRI study done by Rumsey, Donohue, Brady et al. (1997), however, found that all 16 adult right-handed dyslexic male participants had normal asymmetry. This finding suggests that abnormal PT alone cannot be a determinant of developmental dyslexia, in addition to the fact that 
non-dyslexic people have symmetrical and reversed patterns of PT area. As Hier et al. (1978) pointed out, perhaps a combination of structural abnormality (i.e., asymmetrical PT areas) and anatomical malformations (e.g., warts) are required to produce abnormal language function.

Galaburda, Corsiglia, Rosen, and Sherman (1987) reasoned that pathological changes in the level of testosterone during brain development cause abnormal symmetrical and asymmetrical PT formation as well as anomalies in other regions of the cortex, resulting in developmental dyslexia. Galaburda, et al. further speculated that the same developmental processes could enhance some cognitive abilities, such as visuospatial abilities in the RH, which might allow DD children to maintain normal nonverbal intelligence despite their reading disability.

In summary, autopsy and brain scanning studies demonstrated that DD brains are likely to have more symmetry or reversed asymmetry in PT areas than normal brains. These studies also showed that anatomical anomalies (brain warts or cell deformities) particularly in the left PT are often present in DD brains. These findings indicated that a combination of structural abnormality and anatomical anomalies might cause DD.

\subsubsection{Functional Abnormalities}

With the remarkable advance of functional neuroimaging technologies in the last decade, it is not surprising that researchers have used these technologies to study developmental dyslexia. Their research further supports the left hemisphere dysfunction theory. Research using Positron Emission Tomography (PET) (see Appendix D) and functional magnetic resonance imaging ( $\mathrm{AMRI}$ ) (see Appendix E) suggests that DD brains have abnormal brain activation during verbal tasks in the LH (Backes, Vuurman, 
Wennekers et al., 2002; Brunswick et al., 1999; Burton, Small, \& Blumstein, 2000; Corina, Richards, Serafini et al., 2001; Horwitz, Rumsey, \& Donohue, 1998; Milne, Syngeniotis, Jackson, \& Corballis, 2002; Poldrack, Temple, Protopapas et al., 2001; Pugh, Mencl, Jenner et al., 2000; Simos, Fletcher, Bergman, \& Papanicolauou, 2000). For example, in Pugh et al.'s study (2000), brain activation in dyslexic and normal adults was examined using five phonological tasks. These tasks tapped different aspects of functional processing, and the phonological demands were systematically increased. The tasks comprised: (1) a line orientation judgement task as a baseline task (e.g., IIVIV and $\mathrm{V} /$, match?), (2) a letter case judgement task (e.g., bbBb and bbBb, match?), (3) a single letter rhyming task (e.g., $\mathrm{T}$ and $\mathrm{V}$, rhyme?), (4) a nonword rhyming task (e.g., leat and jete, rhyme?), and (5) a semantic category judgement task (e.g., corn and rice, in a same category?). The results showed that typical brain activation was seen in the left posterior temporal regions for the normal readers, whereas little activation was shown in these regions for the DD adults. More interestingly, the normal readers showed an increase in brain activation with increasing phonological processing demands, whereas the dyslexic adults did not; instead they showed greater activation in Broca's area (inferior frontal gyrus) than the normal readers. Normally, brain activation is first seen in Wernicke's and the adjacent areas associated with phonological tasks; however, when a phonological task is complicated, the activation occurs in Broca's area (Burton et al., 2000; Shaywitz, Shaywitz, Pugh et al., 1995). Thus, a question arises as to why the DD adults showed activation in Broca's area without activation in Wernicke's area, and why the Broca's area activation in the DD adults was greater than in the normal readers during the phonological tasks. 
To answer these questions, Pugh et al. (2000) argued that there are the two major brain networks associated with reading, the left posterior regions including Wernicke's area and the anterior regions including Broca's area, and that DD brains have disruption in the left posterior reading systems, resulting in little activation in this area, and that the overactivation in the anterior reading region might be compensation for the disrupted connectivity in the left posterior reading systems. This interpretation was supported by other neuroimaging studies (Fiez \& Petersen, 1993; Paulesu, Frith, Snowling et al., 1995). These studies indicated that the dorsal reading system in the left posteriortemporal region is associated with rule-governed words and non-word reading. Further, the ventral reading system in the left posterior-temporal region has been implicated in whole word reading in the alexia literature (Ellis et al., 1987; Flowers et al., 1991; Friedman et al., 1993; Gross-Glenn et al., 1991; Patterson et al., 1985; Sasanuma, 1974, 1985; Sugishita et al., 1992; Rumsey et al., 1992).

The findings of abnormal activation in dyslexic brains by neuroimaging studies (Backes et al., 2002; Brunswick et al., 1999; Burton et al., 2000; Corina et al., 2001; Horwitz et al., 1998; Milne et al., 2002; Poldrack et al., 2001; Pugh et al., 2000; Simos et al., 2000; Skudlarski et al., 1998) were derived from adult dyslexics. Shaywitz et al. (2002) reasoned that it was necessary to study the brains of DD children to see whether the weak activation of the left posterior temporal regions that was observed in adult dyslexics was the result of a lifetime of poor reading or whether abnormal brain activity was evident during the period of literacy acquisition. Seventy DD children with a mean age of 13 years and their chronological age matched controls were examined by fMRI using the five tasks used by Pugh et al. (2000). The results showed that the DD children 
demonstrated less activation in the left posterior temporal-parieto-occipital regions, which was consonant with the previous studies with adult dyslexics. In the anterior regions including Broca's area, however, normal children showed unilaterally greater activation than DD children. This contradicted the adult data. That is, DD children showed bilateral activation but less than normal readers did, whereas the dyslexic adults in previous studies showed more activation than normal readers unilaterally in this regions. The authors suggested that DD children may compensate for weak connections in the left posterior temporal regions by activating the anterior regions bilaterally just as the adult dyslexics showed greater activation on the left anterior regions than normal readers to compensate for their low activation in the left posterior regions.

In summary, functional neuroimaging studies demonstrated that $\mathrm{DD}$ brains have abnormal brain activation in the anterior and the left posterior brain regions. The findings showed that little activation was present around Wernicke's area and over-activation was seen around Broca's area. It was postulated that DD brains have disruption in the left posterior temporal regions and compensate for this disruption by over-activation in the anterior regions.

\subsection{Summary}

The left hemisphere dysfunction theory claims that dyslexia is caused by abnormal brain organization in the LH. A series of studies with commissurotomized patients found that the two hemispheres are specialized in different cognitive functions; namely language for the $\mathrm{LH}$ and visuo-spatial processing for the $\mathrm{RH}$. The landmark study by Geschwind and Levitsky (1968) demonstrated that $65 \%$ of normal brains have 
a leftward asymmetry PT, and the rest have either a symmetry or rightward asymmetry PT. The PT is located in the temporal lobe, and includes Wernicke's area. It has been speculated that DD brains may have abnormal brain organization in these regions. Autopsy and CT studies found that DD brains have more symmetry or reversed asymmetry PT areas than normal brains. These studies also revealed that DD brains had anatomical anomalies characterized by ectopias or dysplasias. Thus, it was concluded that abnormal brain organization and anatomical anomalies cause DD. MRI studies demonstrated the abnormal brain organization in DD brains to support the left hemisphere dysfunction theory. Furthermore, $\mathrm{AMRI}$ studies found that dyslexic adults and children showed different patterns of brain activation from normal readers, specifically little activation in the left posterior temporal regions and overactivation in DD adults and bilateral activation in DD children in the inferior front regions. These regions are found to be closely associated with reading processing. Taken together, the left hemisphere dysfunction theory claims that the abnormalities in the LH cause developmental dyslexia.

\subsection{Discussion}

The strength of the left hemisphere dysfunction theory is that converging evidence points to the presence of structural, anatomical and functional abnormalities in the LH associated with developmental dyslexia. The functional abnormalities can specifically explain the linguistic deficits that are major impairments in DD children (i.e., phonological processing). Functional neuroimaging studies (Pugh et al., 2000; Shaywitz et al., 2001) and research with alexia (Friedman et al., 1993; Patterson \& Kay, 1982; Patterson et al., 1985; Shallice \& Warrington, 1980) have been suggesting that there are two reading systems in the anterior to posterior temporal regions in the LH. The 
disruption of brain organization in one of these regions could cause difficulties in learning to read.

The left hemisphere dysfunction theory has a number of weaknesses. First, its main weakness is that it cannot account for the fact that not all DD children have abnormal PT areas. In fact, a recent MRI study (Rumsey et al., 1997) provided evidence that all 16 adult DD males who participated in the study displayed normal asymmetry in the PT. Second, the theory does not explain the approximately $35 \%$ of the normal population who have a symmetrical or reversed asymmetrical PT area (Geschwind \& Levitsky, 1968) and who are not dyslexic. As Hier, et al. (1978) pointed out, if it is true that only a combination of abnormal PT and anatomical anomalies could produce abnormal language function, the significant contribution of an abnormal PT to DD is not clear. Further investigation is required to establish the relationship between DD and an abnormal PT. Third, it is not clear how significant the role of testosterone is in DD because DD is found not only in males but also in females (Rumsey et al., 1997). Fourth, the theory totally dismisses other characteristics in $\mathrm{DD}$, such as poor bimanual coordination, poor spatial processing (e.g., telling right or left) or visual problems (e.g., transporting letters, saw $\rightarrow$ was). The left hemisphere dysfunction theory focuses only on the LH. Therefore, it is naturally limited in explaining some deficits demonstrated by DD children, such as poor bimanual coordination, which presumably involves both hemispheres. Finally, the theory has not provided the connection between structural (abnormal PT) and functional abnormalities (typical brain activation patterns around Broca and Wernicke's areas). Further research is required to provide proof of the connection between structural and functional abnormalities in the left posterior regions. 
There is increasing evidence that DD children also exhibit visual processing abnormalities that may be confined to specific parts of the visual system. Much research has suggested that the deficits in a specific neural pathway (the magnocellular pathway) may contribute to reading difficulty by the disruption of accurate letter position information or encoding when it is necessary for efficient word decoding and lexical access (Cornelissen, Richardson, Mason, Fowler, \& Stain 1995). This theory will be reviewed in the next section. 


\section{Chapter 4: The Magnocellular Deficit Theory}

One of the most frequent complaints made by DD children is the phenomenon of moving letters within a word (e.g., saw -> was, pot $->$ top). For this reason, some researchers have speculated that DD children might have impairment in visual processing. The magnocellular deficit theory focuses on the involvement of the basic visual processing system, the magnocellular system, in developmental dyslexia. This theory claims that the abnormalities of the magnocellular system are responsible for DD (Eden, VanMeter, Rumsey et al., 1996; Gross-Glenn, Skottun, Glenn et al., 1995; Livingstone, Rosen, Drislane, \& Galaburda, 1991; Stein \& Walsh, 1997; Talcott, Hansen, Willis-Owen et al., 1998). The chapter will (1) provide a description of the magnocellular and parvocellular systems, and (2) discuss the controversy surrounding the magnocellular deficit theory.

\subsection{Description of the Magnocellular and Parvocellular Pathways}

In all primate visual systems, including that of humans, there are two major systems to process visual information: the magnocellular $(\mathrm{M})$ and parvocellular $(\mathrm{P})$ systems. The two systems have different sensitivities to visual information. The Msystem responds well to motion and depth information, whereas the P-system is sensitive to colour and form (Hubel, 1988; Livingstone \& Hubel, 1988). The two systems are segregated from each other from the retina to the primary visual cortex (V1) through the lateral geniculate of the nucleus (LGN) (Figure 4.1). This distinct visual organization 
occasionally results in selective visual impairments, such as loss of motion with preserved color perception (Livingstone \& Hubel, 1988).

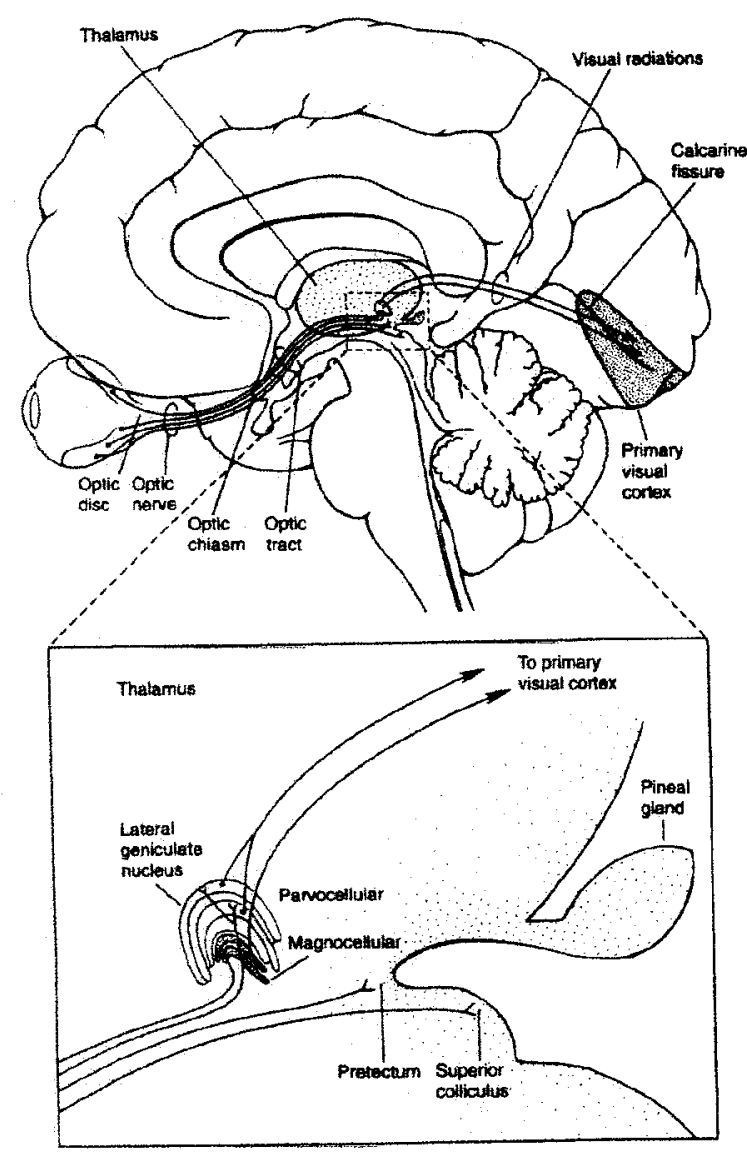

Figure 4.1. The visual pathways from the retinal to the primary visual cortex through the thalamus A simplified diagram of the projections from the retina to the various visual areas of the thalamus (lateral geniculate nucleus), midbrain (pretectum and superior colliculus), and area 17 of the cerebral cortex. The parvocellular and Magnocellular are segregated from the retinal to the primary visual cortex (Mason \& Kandel, 1991).

As shown in Figure 4.2, the M-system axons terminate in the occipital cortex in layer 4C $\alpha$ and 4B in area V1, and the P-system axons terminate in layer 4C $\beta$ in area V1. 
Beyond the V1, the M- and P-systems are not completely separated. Nevertheless, the Msystem predominantly sends axons to area 19 in the secondary visual cortex (MT/V5) and terminates in the parietal lobe via a dorsal stream projection, which is specialized for the analysis of movement and stereoscopic depth (Kandel, 1991). The P-system, which interconnects with the M-system at area 19 and terminates at the inferior temporal cortex via a ventral stream projection, carries colour and form information (Livingstone \& Hubel, 1988).

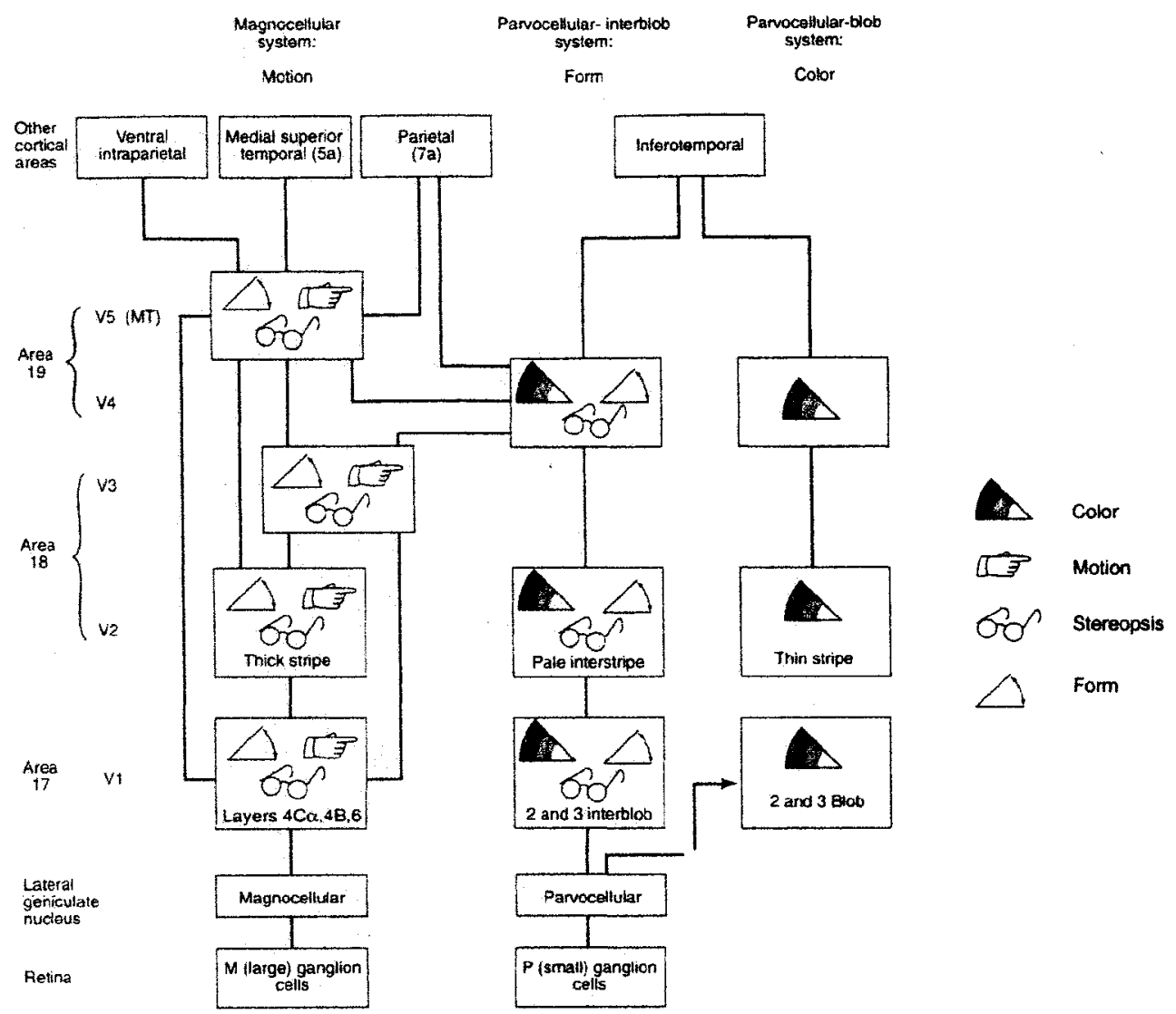

Figure 4.2. Parallel visual pathways of the Magnocells and Parvocells and their suggested functions in the macaque monkey (Adapted from DeYoe and Van Essen, 1988). 
There are four main aspects that differentiate the M- and P-cells (Shapley, Kaplan, \& Soodak, 1981). First, the M-cells are sensitive to lower spatial and higher temporal frequencies, whereas the P-cells are sensitive to higher spatial and lower temporal frequencies. Second, the M-cells are mainly located at the periphery, whereas the P-cells are predominantly located around the fovea, i.e., the M-cells are specialized for coarse vision and the P-cells are specialized for acute vision. Third, the M-cells respond to stimuli faster and more transiently than the P-cells. Finally, the axons of the M-cells are larger and more heavily myelinated than the P-cells (Talcott et al., 1998). More specifically, the M-cells rapidly respond to a 1-2\% difference in brightness contrast and level off at about 10-15\% difference, whereas the P-cells respond slowly at the start and level off at far higher contrasts. In sum, the M-cells are colour blind, but have high sensitivity to motion and coarse visual features, whereas the P-cells are sensitive to colour and to the details of stationary objects (Livingstone \& Hubel, 1988).

During reading our eyes are constantly and quickly moving to fixate from one point to another to scan visual presentation. This eye movement is called a saccade. During each fixation, the reader extracts visual information from the printed text and saccades take the reader forward through the text (Ellis, 1994). Although the role of the M-cells in reading is unknown, Lovegrove, Garzia and Nicholson (1990) suggested that the M-cells have a role in inhibiting the P-cells after each saccade in erasing the image, as otherwise the image would persist on the previous fixation, and letters and words would likely be "smeared". This inhibition is called saccadic suppression. Since the Msystem is closely related to eye movement during reading, it logically follows that deficits in the M-system might cause developmental dyslexia. In fact, recent research has 
confirmed that some adult dyslexics have an impairment in the M-system (Cornelissen et al., 1995; Demb, Boynton, Best, \& Heeger, 1998; Demb, Boynton, \& Heeger, 1997; Eden et al., 1996; Kubova, Kuba, Peregrin, \& Novakova, 1995; Talcott et al., 1998; Vidyasagarr \& Pammer, 1999). Although evidence for M-cell deficits in DD has been accumulating, the involvement of visual deficits in DD has not yet been widely accepted. Thus, there is controversy surrounding the magnocellular deficit theory. The next section will discuss this issue.

In summary, there are two distinct major visual systems from the retina to the primary visual cortex. The magnocellular system is sensitive to lower spatial and high temporal frequencies, i.e., it is specialized for motion and depth detection. In contrast the parvocellular system is sensitive to high spatial and low temporal frequencies, i.e., it is specialized for colour and shape detection. It has been proposed that DD is associated with a deficit in the M-system.

\subsection{The Controversy Surrounding the Magnocellular Deficit Theory}

The main controversy surrounding the magnocellular deficit theory is that although more than $75 \%$ of adult dyslexics have impairment in the M-system, some do not (Lovegrove et a., 1986). For example, a visual evoked potential (VEP) study (Livingstone et al., 1991) found that contrast sensitivity and temporal resolution were slower in five dyslexics (mean age of 27.4 years) than seven normal (mean age of 25.8 years) groups. An evoked potential method measures a regular pattern of electrical activity recorded from cells evoked by a controlled visual stimulus (Reber, 1985). In the study, a checkerboard was used as a stimulus to measure spatial frequencies at high and low luminance contrast. The results showed that at the low spatial contrast (M-system), 
the VEP peak in the DD group appeared with a 20 to $40 \mathrm{msec}$ delay from the peak in the normal group, whereas at the high spatial contrast (P-system), both VEP patterns looked similar. Livingstone et al. interpreted this finding such that dyslexic adults have an impairment in the M-system, resulting in this slow magnocellular activity. The authors argued that the deficiency of sensitivity in the M-cell system causes difficulty in detecting low contrast between the letters and background, which makes reading difficult for individuals with dyslexia.

Furthermore, Livingstone et al. (1991) examined the LGN in autopsy specimens from five dyslexic brains (four males and one female, mean age of 34.2 years) and five nondyslexic brains (all males: mean age of 40 years). They found that the P-system looked similar in both brains, but the M-system was more disorganized and the cell bodies appeared smaller in the dyslexic than the normal brains. Thus, Livingstone, et al. argued that dyslexia might be related to perceptual problems as a result of the disturbance of the M-cells, in spite of the fact that it is widely thought to be related to linguistic deficits. Cornelissen et al. (1994) also provided evidence that DD adults were significantly less sensitive to motion. This lower sensitivity to motion in DD adults was also reported by other studies, such as a VEP study (Kubova et al., 1995), an MRI study (Best \& Demb, 1999), and AMRI studies (Demb et al., 1997; Eden, et al. 1996).

Demb et al. (1998) examined saccadic suppression using different spatial frequencies and found a positive correlation between reading rate and accurate performance on a coherent motion detection task, in which the participants are asked to detect whether or not the total number of dots move in a single direction as a set. These 
positive findings suggest that the magnocellular deficits demonstrated in most dyslexics might contribute to DD (Martin \& Lovegrove, 1987; Talcott et al. 1998).

Despite these positive findings, there have also been negative findings regarding the magnocellular deficit theory. Two studies (Johannes, Kussmaul, \& Mangun, 1996; Victor, Conte, Burton, \& Nass, 1993) failed to replicate the VEP study by Livingstone, et al. (1991). Gross-Glenn et al. (1995) found that adult dyslexics did not differ from their controls in the ability to detect transient stimuli and reduce contrast sensitivity to high but not low spatial frequency. These researchers argued that dyslexics appear to have a deficit in the P-system and not the M-system because the P-system is sensitive to high spatial frequency. A recent study (Keen \& Lovegrove, 2000) provided another negative result in a study of retinal sensitivity. Since the M-cells are abundant in the periphery and the P-cells are dominant around the fovea, the authors suggested that the M-cells process a global image rather than the details of parts, and that the P-cells are specialized for the details of a local image rather than a whole image. Keen and Lovegrove examined retinal sensitivity in dyslexic children (mean age of 10 years), as well as in the chronological age-matched (CA) and reading age-matched (mean age of 7.3 years) groups. The results showed that there was no difference between groups in the processing of global and local elements of spatial stimuli. This implies that DD children have no deficit in the M-cells.

A crucial negative finding came from a study by Burr, Morrone, and Ross (1994). They examined saccadic suppression using different spatial frequencies and found that the saccadic suppression occurred at low spatial frequencies, whereas the sensitivity at high spatial frequencies was unchanged. The authors suggested that during saccade the M-system selectively suppresses itself. This finding contradicts the logic of the 
magnocellular deficit theory with respect to the putative role of the M-cells in suppressing the P-cells during saccades to prevent eye movement. Thus, Skottun (1997) criticized the magnocellular deficit theory based on saccade suppression. Skottun (2000) further argued that the theory missed an important point, i.e., how deficits in the Msystem, which responds to rapidly moving stimuli, are related to stationary printed stimuli. He suggested that the magnocellular deficit theory needs to provide a convincing explanation as to how the M-system is related to reading difficulties.

As noted in the previous section, about $75 \%$ of adult dyslexics demonstrated an M-system deficit (Lovegrove et al., 1986). Borsting, Ridder, Dudeck, et al. (1996) found that the presence of the magnocellular deficit depended upon the type of developmental dyslexia. In their study (1996), DD children were sub-grouped based on the Boder's subtypes system classification $(1971,1973)$ (see Chapter 1-3) in order to compare dyseidetic and dysphoneidetic dyslexics to normal age-matched controls in terms of sensitivity to spatial frequency at low and high temporal contrasts $(1 \mathrm{~Hz}$ and $10 \mathrm{~Hz})$. They found that the dyseidetic dyslexics did not differ in a broad range of spatial frequencies at both contrasts, whereas the dysphoneidetic dyslexics demonstrated a reduction in sensitivity to low spatial frequency stimuli at high temporal contrast. The results suggest that only the 'phonological' (dysphoneidetic) type of dyslexia showed the deficit but 'visual' types of dyslexia (dyseidetic) did not show a defect in the magnocellular system. This is partially consistent with the magnocellular deficit theory, which claims that dyslexics are poor in quick motion. Thus, the researchers argued that the presence of the magnocellular deficit might be related to the sub-type of developmental dyslexia. Although the results were supported by other studies (Ridder, Borsting, Cooper, McNeel, \& Hauing, 1997; 
Slaghuis \& Ryan, 1999), recent studies, including one by the same researchers who found the positive results, found no different motion and contrast sensitivity in the Mcells in the sub-types of dyslexia (Ridder, Borsting, \& Banton, 2001; Williams, Stuart, Castles, \& McAnally, 2003). This implies that the relationship between the sub-type of dyslexia and the M-system deficit is not yet conclusive.

In relation to the abnormal asymmetrical planum temporale (PT) in DD children discussed in Chapter 3, Best and Demb (1999) found that the magnocellular deficits in dyslexia might exist independently from abnormal PT asymmetry in dyslexia. Since abnormal asymmetrical PT and the magnocellular deficit were documented in previous studies, Best and Demb examined the relationship between abnormal asymmetry in the PT area and a deficit in the M-system in dyslexia. All five dyslexic subjects (mean age of 22 years) were diagnosed with dyslexia in childhood and showed reduced brain activity relative to the controls in the V1 and MT+ (M-system) in a previous fMRI study (Demb et al., 1998). In Best and Demb's study, the volume of the PT areas was measured using MRI. The results showed that three out of the five dyslexic subjects had normal leftward symmetrical plana. Best and Demb argued that since all subjects in the study demonstrated phonological deficits, it is possible that a different sub-type of dyslexia is specifically related to PT abnormality and that these two neurological markers for dyslexia could be independent.

In summary, there are pros and cons regarding the magnocellular deficit theory. The positive findings are that dyslexic brains in autopsy studies showed smaller cell bodies in the M-cells, whereas no difference was found in the P-cells, and dyslexics showed less sensitivity to low spatial frequencies and motion detection. The negative 
findings were demonstrated in no difference between the dyslexic group and the controls in low spatial frequency detection in one study (Gross-Glenn et al., 1995). Burr, et al. (1994) found that the M-system, but not the P-system, selectively suppresses itself during saccades. Another negative finding is that researchers have yet to find a link between Mcell deficits and abnormal PT area. However, although the magnocellular deficit theory may not be grounded in firm logic, evidence continues to accumulate for this theory.

\subsection{Summary}

The two main visual systems, the magnocellular and parvocellular systems, are segregated from the retina to the primary visual cortex (V1). The M-cells are sensitive to motion and depth and the P-cells are sensitive to colour and form (Livingstone \& Hubel, 1988). There is evidence indicating that developmental dyslexics have impairment in the magnocellular system but not the parvocellular system. The dyslexic brain typically has smaller cell bodies in the M-cells than does the normal brain (Livingstone et al., 1991), slow brain activity in VEP (Kubova et al., 1995; Livingstone et al., 1991), less brain activity to motion in fMRI (Demb et al., 1998; Eden et al., 1996), and less sensitivity to low spatial frequency stimuli (Cornelissen et al., 1995; Demb et al., 1998; Stein \& Walsh, 1997; Talcott et al., 1998). These findings support the claim that a magnocellular deficit causes reading disabilities in dyslexia.

Despite the positive evidence, negative results have also been reported: two failures to replicate the VEP study of Livingstone, et al. (1991) (Johannes et al., 1996; Victor et al., 1993) and a finding in favor of a parvocellular deficit (Gross-Glenn et al., 1995). Among the negative results, a finding by Burr, et al. (1994) was crucial, i.e., these authors' findings demonstrated that the M-system has no role in inhibiting the P-system 
during saccades; rather, the M-system inhibits itself by a feed forward mechanism to prevent eye movement. In addition, the view that the magnocellular deficit may differentiate a sub-type of dyslexics is not conclusive, because the findings were not consistent, i.e., some were positive (Borsting et al., 1996, Ridder et al., 1997; Slaghuis \& Ryan, 1999), and some not (Ridder et al., Williams et al., 2003). Further, adult dyslexic subjects in Demb, et al.'s study (1998) who had a deficit in the M-system showed normal asymmetry in the PT area, suggesting that these two abnormalities in dyslexia may be independent.

\subsection{Discussion}

The major strength of this theory is that the evidence that most dyslexics have impairment in the M-system has been accumulating (Cornelissen et al., 1994; Demb et al., 1997; Eden et al., 1996; Livingstone et al., 1991; Talcott et, al., 1999). This theory can provide a good explanation for the problem of letter transporting in words, which is one of the complaints made by DD children. The major weakness of this theory is that some studies failed to replicate the positive results of magnocellular deficit in dyslexia and that it does not provide a clear explanation of the relationship between the M-cell system and reading. It was believed that the M-system suppresses the P-system during saccade to prevent different fixations of images. This turned out, however, not to be true (Burr et al., 1994). This is a critical negative finding for the theory, because the theory was based on the logical assumption that the M-system inhibits the P-system during saccade to erase an image. As Skottun (2000) pointed out, the magnocellular deficit theory needs to investigate direct and indirect connections with reading. In addition, Best and Demb (1998) found that the magnocellular deficits in DD might exist independently 
from abnormal PT asymmetry in dyslexia. This finding made the magnocellular deficits theory appear weak because the presence of the M-cell deficit seemed less significant in developmental dyslexia. Another weakness of this theory is that most previous studies were done with adult dyslexic subjects (Cornelissen et al., 1994; Eden, et a., 1996; Gross-Glenn et al., 1995; Livingstone et al., 1991; Reber, 1985; Talcott et al., 1998). Research with children needs to be included to investigate how DD manifests itself in different developmental stages. Because the theory focuses on one of the multiple brain regions associated with $\mathrm{DD}$, it is limited in its ability to account for other deficits of DD, including poor phonological processing, poor bimanual coordination, a lack of fluency, and/or poor sequence processing.

The three theories previously discussed have a common feature. That is, each theory attempts to account for developmental dyslexia within restricted brain regions (i.e., the PT areas, the visual cortex, subcortical areas) or specific brain functions (i.e., phonological processing and visual processing). In contrast, the fourth theory, to be discussed in Chapter 6, accommodates the two cerebral hemispheres (i.e., the entire cortex) in attempting to understand reading problems. Interhemispheric interaction involves the corpus callosum $(\mathrm{CC})$, which is the major brain structure connecting the two cerebral hemispheres. The fourth theory, the interhemispheric disconnection theory, focuses on the involvement of the $\mathrm{CC}$ in developmental dyslexia. First, however, the structures and functions of the $\mathrm{CC}$, and disconnection syndromes resulting from damage to the $\mathrm{CC}$, will be discussed in the next chapter. 


\section{Chapter 5: The Corpus Callosum}

The corpus callosum (CC) is a large midline brain structure connecting the two cerebral hemispheres. It is present in most animals from insectivores to higher primates (Oivares, Michalland, \& Aboitiz, 2000). It appears that the CC has evolved in parallel with the neocortex. A recent cross-species morphometric study (Olivares et al., 2000) found that callosal size was correlated with brain weight across species.

Despite over a century of research, the function of the $\mathrm{CC}$ is not yet fully understood. However, studies with patients with commissurotomy (sectioning of the CC to prevent spreading of epileptic seizures to the opposite hemisphere) and children with callosal agenesis (congenital absence of the $\mathrm{CC}$ ) have greatly contributed to unfolding the functions of the $\mathrm{CC}$. It is assumed that functioning of the $\mathrm{CC}$ may be related to anatomical and functional laterality, e.g., language for the left hemisphere (LH) and visuo-spatial processing for the right hemisphere $(\mathrm{RH})$, and may contribute to interhemispheric interaction.

The chapter will discuss four topics: (1) the anatomy of the CC; (2) research background; (3) the functions of the callosum; and (4) the CC, gender and handedness. In the first section, a brief review of the neuroanatomical structures of the $\mathrm{CC}$ will be presented. 


\section{1 Anatomy of the Corpus Callosum}

In the human brain, the two hemispheres are connected by bundles of nerve fibers called the commissures. The major commissures are the $\mathrm{CC}$, the anterior, the hippocampal, and the posterior commissures (see Figure 5.1). Among them, the largest commissure is the $\mathrm{CC}$, which connects the cerebral hemispheres. The rest of the commissures subcortically connect the two hemispheres (Banich, 1997).

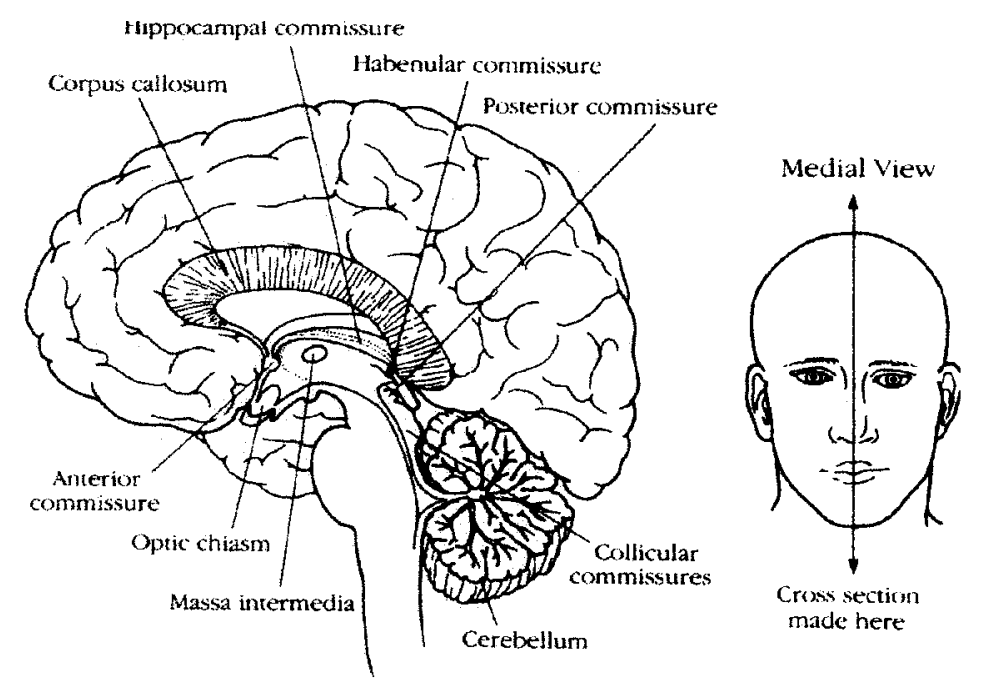

Figure 5.1. The position of the CC compared with the locations of other brain commissures. (Sperry, 1964)

It is estimated that there are approximately 200 million callosal fibers in the CC and most are myelinated (Tomasch, 1954). Callosal fibers connect the two cerebral hemispheres in a specific way, i.e., three quarters of the callosal fibers connect homologous regions and the rest connect heterogeneous regions of the two hemispheres (Cook, 1986). The connection is achieved by a columnar unit containing 1,000 to 
100,000 neurons instead of a one-to-one axonal connection between the cerebral hemispheres (Cook, 1986; Pandya \& Seltzer, 1986).

The topographical organization of the callosal fibers is specific (De Lacoste, Kirkpatrick, \& Ross, 1985; Pandya \& Seltzer, 1986). Structurally, the anterior regions of the $\mathrm{CC}$ connect to anterior areas of the cerebral cortex (i.e., the frontal lobe) and the posterior callosal regions connect to the posterior areas of the cerebral cortex (i.e., the temporal-parietal and occipital lobes). Because of this organization, it was assumed that different types of information were transferred via specific regions of the $\mathrm{CC}$ (De Lacoste et al., 1985). For example, Broca's area (involved in speech production) is connected via the genu to the homologous area in the $\mathrm{RH}$, motor information is processed via the anterior midbody, somato-sensory information is transferred through the posterior midbody, the isthmus connects Wernicke's language area to the opposite homologous area, and visual information is transferred through the splenium. Recent MRI and PMRI studies have confirmed that the splenium transfers exclusively visual inputs (Fabri, Plonara, Quattrini et al., 1999; Funnell, Corballis, \& Gazzaniga, 2000) (see Figure 5.2).

It must be noted that measurement of the $\mathrm{CC}$ is not definitive and there is controversy surrounding it. Although details of the controversy are beyond the scope of this paper, some researchers divided the $\mathrm{CC}$ into three large portions, and then further subdivided them into small regions (Aboitiz, Scheibel, Fisher, \& Zaidel, 1992). Others divided it equally into six regions (Moffat, Hampson, \& Lee, 1998). Division has been based on various principles. In this section (see Figure 5.2), the measurement system developed by Witelson (1989) divided the CC into seven sections using an autopsy, was 
used to illustrate the callosal organization. Witelson's division was consistent with another study using a computer analysis to divide the CC (Denenberg, Kertesz, \& Cowell, 1991), and is the most widely accepted by researchers (Giedd, Blumenthal, Jeffries et al., 1999; Giedd, Rumsey, Castellanos et al., 1996; Stancak, Lucking, \& Kristeve-Feige, 2000; Steinmetz, Jäncke, Kleinschmidt et al., 1992).

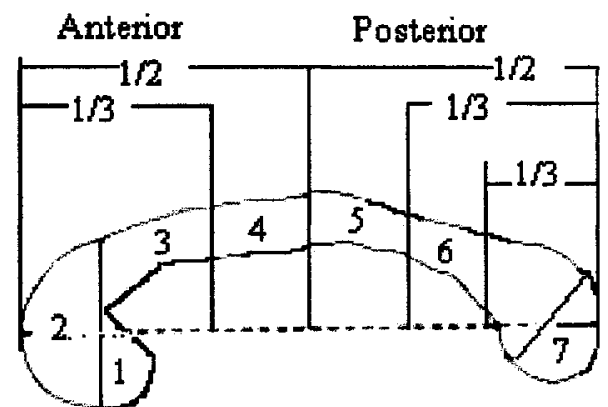

Figure 5.2. Diagram of the midsagittal view of the $\mathrm{CC}$ of the human adult. It shows the seven regional subdivisions used for area measurement, numbered 1-7. 1 (Rostrum, prefrontal, inferior premotor), 2 (Genu, prefrontal), 3 (Rostral body: premotor, supplementary motor), 4 (Anterior midbody: motor), 5 (Posterior midbody: somaesthetic, posterior parietal), 6 (Isthmus: superior temporal, posterior parietal), and 7 (Splenium: occipital, inferior temporal) (Witelson, 1989).

There is evidence that different callosal regions are composed of different fibers in terms of density and size. A study (Aboitiz et al., 1992) using light microscopic examination revealed that small fibers with a diameter larger than $0.4 \mu \mathrm{m}$ tend to be clustered in the genu (corresponding to 2 in Figure 5.2). Large fibers with a diameter of 3 to $5 \mu \mathrm{m}$ are concentrated in the midbody (corresponding to $4 \& 5$ in Figure 5.2), and middle sized fibers with diameters larger than $1 \mu \mathrm{m}$ are most frequently seen in the isthmus (corresponding to 6 in Figure 5.2) and splenium (corresponding to 7 in Figure 
5.2). Why the fibers are so different in these callosal regions is still not known. The authors of this study suggested that the large callosal fibers in the midbody connecting auditory regions might contribute to the production of an auditory neural map because bilateral auditory inputs are important in sound localization, and thus they may require interhemispheric interaction for timing. The authors further suggested that fine callosal fibers mostly concentrated in the genu and isthmus seem to be related to higher-order processing because they connect two language areas.

In summary, the $\mathrm{CC}$ connects the homologous and heterogeneous areas in the two cerebral hemispheres. It is organized in an orderly manner from the anterior to the posterior; and it is currently believed that each region is associated with different cortical functions. The different composition and density of callosal fibers appears to be closely related to callosal topography. Relations between the types of callosal fibers and callosal functions in interhemispheric interaction are not yet well understood.

For more than a century, clinical and animal studies have attempted to understand the function of the CC in brain processing. Until the mid-1950s, no study had demonstrated evidence that the $\mathrm{CC}$ played a significant role in interhemispheric interaction. However, in 1958, Myers and Sperry (1958) provided evidence of this in a cat. The next section will briefly describe the research history of the CC.

\subsection{Research Background}

From the nineteenth to the mid-twentieth century, it was postulated that the $\mathrm{CC}$ must have some significant roles in mental functions (Gordon, 1974) because of its massive size and central location in the brain. This callosal hypothesis assumed that 
some types of cognitive or behavioural impairment would occur when the $\mathrm{CC}$ was surgically sectioned. However, sectioning the $\mathrm{CC}$ had little effect on commissurotomized patients' daily lives (Akelaitis, Risteen, Herren, \& Wagene, 1942; Bridgman \& Smith, 1940; Dandy, 1936). For example, Dandy (1936) reported three ventricle tumor patients (a 10 year-old boy, 15 year-old boy and 28 year-old woman), whose posterior corpus callosa were sectioned. These patients showed no effect from the surgery. Dandy concluded that the CC had no function. Later, Akelaitis et al. (1942) also tested the cognitive functions of epileptic patients whose corpus callosa were partially or completely sectioned. These patients also showed few obvious cognitive or perceptual changes. Thus, the authors dismissed the function of the CC.

Despite these negative results, Van Wagenen and Herron (1940) noted that a patient complained of a lack of coordination between his left hand and the rest of his body, and Akelaitis (1942) also reported a conflict between the left and right hands shown by his patients. Although these symptoms are now known as 'disconnection' effects, a term which refers to behavioural deficits caused by damage of information transmission from one hemisphere to another (Banich, 1997), they were then dismissed by the researchers because the symptoms were temporary. Subsequent animal experiments with monkeys (Lashley, 1950) did not show any behavioural disturbances after sectioning the $\mathrm{CC}$. These successive failures from clinical and animal studies led researchers to the conclusion that the $\mathrm{CC}$ had little connection with mental function (Bridgman \& Smith, 1945).

Myers and Sperry (1958) first demonstrated disconnection effects in a visuosensory transfer experiment in a cat using a tachistoscopic technique (see Appendix A). 
When visual material is briefly (for less than $200 \mathrm{msec}$ ) presented by a tachistoscope either in the left or right visual field, the information directly projects in the contralateral primary visual cortex via the optic chiasm in each hemisphere, i.e., a stimulus presented in the left visual field projects the image in the $\mathrm{RH}$. The information is then transferred to the opposite hemisphere via the CC (see Figure 5.3). When Myers and Sperry sectioned both the optic chiasm and the $\mathrm{CC}$ of a cat, the result was remarkable. The cat, which had been trained to do a discrimination task with one eye before sectioning, failed to perform the same task after sectioning of the $\mathrm{CC}$ when required to use the other untrained eye, i.e., information was not transferred to the opposite hemisphere after sectioning of the $\mathrm{CC}$ and the optic chiasm. This landmark study explicitly illustrated that it is impossible to transfer visual information to the opposite hemisphere when both the $\mathrm{CC}$ and optic chiasm have been sectioned. The study clearly indicated that the $\mathrm{CC}$ plays an important role in the interhemispheric transfer of visual information. Berlucchi (1990) commented that the consecutive failures in showing disconnection effects in earlier times were mainly due to inadequate methodologies, such as partial sectioning of the CC.

Following this study, many experiments demonstrated that sectioning the CC hinders interhemispheric information transfer in a variety of animals, such as macaques (Sperry, 1961), chimpanzees (Black and Myers, 1964), fish (Doty \& Negrao, 1973) and birds (Goodale \& Graves, 1982). Patients with commissurotomy were also tested by a tachistoscopic technique. The results demonstrated disconnection effects (Bogen \& Vogel, 1962; Gordon \& Sperry, 1969; Levy et al., 1972; Sperry, 1968, 1974; Sperry et al., 1969). For example, commissurotomized patients could name an item flashed in the right visual field (RVF)/LH, but when the same item was presented in the left visual field 
(LVF)/RH, they could not name it. This is because the item presented in the LVF projects in the RH first, and then needs to be transferred to the LH for verbalization due to language specialization in the $\mathrm{LH}$. However, when the $\mathrm{CC}$ is sectioned, the information cannot be transferred to the $\mathrm{LH}$, and thus, the disconnection effect appears (Bogen \& Vogel, 1962). These early commissurotomized studies with humans and animals demonstrated the significant role of the $\mathrm{CC}$ in information transfer from one hemisphere to another.

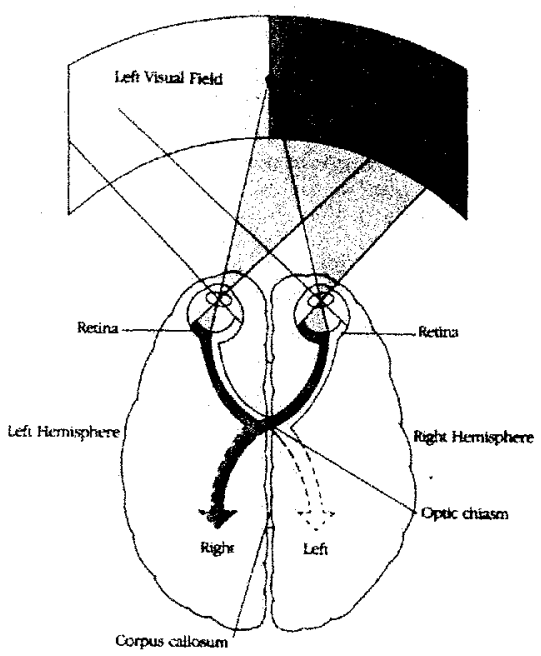

Figure 5.3. Visual pathways to the cerebral hemispheres.

When fixating on a point, each eye sees both visual fields but sends information about the right visual field only to the left hemisphere and information about the left visual field only to the right hemisphere. This crossover and split are a result of the manner in which the nerve fibers leading from the retina divide at the back of each eye. The visual areas of the left and right hemisphere normally communicate through the $\mathrm{CC}$. If the callosum is cut and the eyes and head are kept from moving, each hemisphere can see only half of the visual world (Springer \& Deutsch, 1989). 
The next section will discuss the current beliefs about functions of the $\mathrm{CC}$ in humans. The section will focus on four topics: (1) callosal transfer, (2) motor coordination, (3) integration, and (4) language. These topics are closely related to the present study with developmental dyslexia.

\subsection{The Functions of the Corpus Callosum}

Considering the fact that the $\mathrm{CC}$ connects mainly homologous regions of the two cerebral hemispheres, it could be assumed that the two brains somehow communicate through the CC. Hemispheric specialization tells us, on the other hand, that the two brains work independently in some cognitive tasks, such as language and spatial processing. How do they communicate with each other and at the same time carry out independent tasks? What is the role of the $\mathrm{CC}$ in interhemispheric interaction?

Sperry (1962) first postulated an excitatory role for the CC because commissurotomized cats demonstrated failure of visual information transfer from one hemisphere to another. According to the excitatory hypothesis, information, no matter which hemisphere receives it, has to be transferred to the opposite hemisphere. Thus, the main role of the $\mathrm{CC}$ is seen as information transfer (callosal transfer). As a result of information transfer, the two hemispheres receive duplicated information through the CC. A question pertaining to this hypothesis is why duplicated information is needed in brain processing. Although Sperry did not elaborate on this question, the view of callosal transfer is still valid (Cook, 1986). Zaidel (1995) conceptualized the role of the CC in terms of both excitatory and inhibitory mechanisms. His view is as follows: sometimes each hemisphere processes information independently, and in parallel; at other times, the 
two hemispheres exchange the results of independent computation for utilization of information. However, when one hemisphere dominates the other in processing, the utilization of the information needs to be inhibited from the other hemisphere, for example, phonological processing for the $\mathrm{LH}$, or visuo-spatial processing for the $\mathrm{RH}$. Thus, the CC has an excitatory role for transferring some information between the two hemispheres, while at the same time having a role of inhibition for allowing one hemisphere to operate independently from the other in a task (i.e., hemispheric specialization). This conceptualization fits well with the results from an electrophysiological study (Toyama, Tokashiki, \& Matsumi, 1969). This study demonstrated that when callosal fibers were stimulated in a cat brain, they showed initially excitatory responses followed by prolonged inhibitory responses at the site of termination of callosal fibers.

The next section will discuss the role of the $\mathrm{CC}$ in information transfer by providing a brief review of studies with commissurotomized patients and children with callosal agenesis.

Children with callosal agenesis have provided insight into the functions of the CC in a different way than have commissurotomized patients. Studies with commissurotomized patients have demonstrated disconnection effects in the adult brain. Typically, disconnection effects have been interpreted as loss of brain functions, while studies with agenesis of the $\mathrm{CC}$ have provided evidence of cerebral plasticity.

The etiology of callosal agenesis is heterogeneous and multifactorial, and callosal agenesis is often accompanied by other neurological deficits that cause low general psychological functioning; in fact, it is associated with more than 50 neurological 
disorders. The most common genetic disorder is Andermann syndrome (Geoffroy, 1994; Wisniewski \& Jeret, 1994). Although many children with callosal agenesis demonstrate mental retardation, seizures, or motor impairment, these symptoms are nonspecific to callosal agenesis and there is no specific clinical symptom for callosal agenesis as there is for commissurotomy. Callosal agenesis used to be accidentally found at autopsy, but recently, agenesis of the $\mathrm{CC}$ has been found by modern scanning methods, such as the CT scan (Larsen \& Osborn, 1982), MRI (Davidson et al., 1985; Han, Benson, Kaufman et al., 1985), ultrasonography (Hernanz-Schulman et al., 1985), and prenatal fetal ultrasound examination (Sandri et al., 1988; Hilpert \& Kurtz, 1990).

The next section will discuss the role of the $\mathrm{CC}$ in information transfer with two special populations, commissurotomized patients and children with callosal agenesis.

\subsubsection{Callosal Transfer}

As previously noted, early studies (Bogen \& Vogel, 1962; Gordon \& Sperry, 1969; Sperry, 1968, 1974; Sperry et al., 1969) revealed that commissurotomy disrupted visual information transfer between two hemispheres. Although commissurotomized patients had no difficulty in identifying an object presented in the LVF/RH with their left hands and in the RVF/LH with their right hands (Uncrossed condition), when they were asked to identify an object with the LVF/RH and right hand or the RVF/LH and left hand (Crossed condition), a disconnection effect appeared (Sperry, 1968, 1974; Sperry et al., 1969; Zaidel, 1983). Sperry explained this such that information presented in the LVF/RH first projects in the $\mathrm{RH}$ and needs to cross the $\mathrm{CC}$ to travel to the $\mathrm{LH}$ in order to name the item (Crossed Condition), because the LH is specialized for language for most 
people, whereas an object presented in the $\mathrm{RVF} / \mathrm{LH}$ projects directly into the $\mathrm{LH}$ (Uncrossed condition). Thus, on the Crossed condition, information is disrupted due to callosal sectioning. Similarly, Sperry (1968) examined a commissurotomized patient, NG, using a tachistoscopic technique. NG was asked verbally to identify an object briefly flashed in either her LVF/RH or RVF/LH. She could name the object when it was flashed in her RVF/LH, but when the same object was flashed in her LVF/RH, she failed to answer. Sperry also tested NG in a series of tactile tasks. In one of the tasks, NG was asked to verbally identify an object that she touched without viewing. NG could not name the object when she touched it with her left hand/RH (Crossed condition). However, as soon as she held the same object in her right hand/LH (Uncrossed condition), she could name it without viewing it. These results suggest that the $\mathrm{CC}$ plays a role in interhemispheric tactile information transfer.

Another tactile study (Geffen, Nilsson, Quinn, \& Teng, 1985) confirmed impairments in tactile information transfer in commissurotomized patients. In this study, six partially commissurotomized patients (i.e., with partial sectioning of the $\mathrm{CC}$, in these cases, with sectioning of the genu or midbody) and four completely commissurotomized patients were compared to 24 normal subjects in a finger localization task. The task involved imitating finger sequences on the same or opposite hand when fingers were sequentially touched without viewing. An important point in this study was that these researchers hypothesized that callosal transfer would be required when the opposite hand was used for the imitation, whereas it would not be required for same hand imitation. The results showed that all three groups performed perfectly on the Uncrossed condition (same hand); however, on the Crossed condition (opposite hand), the results differed 
depending on the severity and location of the sectioning. The performance of the completely commissurotomized patients in this condition declined by $82 \%$, the performance of patients with sectioning in either the anterior or posterior midbody (corresponding to $4 \& 5$ in Figure 5.2) showed a decline of $28 \%$, and the patient with sectioning of the genu (corresponding to 2 in Figure 5.2) showed a $4 \%$ decrease compared to the controls who showed a $7 \%$ decrease. These differences were statistically significant except for the patient with sectioning of the genu. These findings implied that the $\mathrm{CC}$ facilitates not only the interhemispheric transfer of simple tactile information but also transfers specific information through a specific region, i.e., tactile sensory information is transferred through the anterior and posterior midbody of the CC.

Other examples of disconnection effects observed in this population were lefthand apraxia (inability to respond to verbal commands) (Aglioti, Beltramello, Tassinari, \& Berlucchi, 1998; Watson \& Heilman, 1983; Zaidel \& Sperry, 1974) and poorer performance of the right than the left hand in copying geometric figures (Loring, Meador, \& Lee, 1989; Zaidel, \& Sperry, 1974). The most common explanation for lefthand apraxia is as follows (Watson \& Heilman, 1983). The LH is specialized for language for most people, and thus the $\mathrm{LH}$ understands a verbal command. To follow the verbal command, the information needs to be transferred from the $\mathrm{LH}$ to the $\mathrm{RH}$ via the $\mathrm{CC}$ because the $\mathrm{RH}$ controls motor movement of the left hand; however, the information cannot be transferred to the $\mathrm{RH}$ in the case of callosal sectioning or lesions. In contrast, in terms of copying geometric figures, the geometric information needs to be transferred from the $\mathrm{RH}$ to the $\mathrm{LH}$ because the $\mathrm{RH}$ is specialized for visuo-spatial processing, but this is interfered with due to callosal sectioning. 
Unlike commissurotomized patients, individuals with callosal agenesis can typically name a visual stimulus (e.g., letters) presented to the LVF/RH and can also compare linguistic stimuli presented bilaterally ('cross-matching') (Corballis \& Finlay, 2000). It has been argued that bilateral speech representation may explain why this population can name an object in the LVF/RH (Sperry, 1968; Denenberg, 1981). Bilateral speech representation, however, cannot account for their ability to 'crossmatching' (Milner, 1994). Milner argued that individuals with callosal agenesis can transfer visual information interhemispherically via alternative pathways, probably via the subcortical structures, which seem not to be available to commissurotomized patients.

To test the alternative pathway theory, a reaction time (RT) paradigm was used. Studies using an RT paradigm with normal adults (Jeeves, 1969; Milner \& Lines, 1982) found that interhemispheric information transfer normally takes 2-3 msec longer than intrahemispheric information processing. For example, a subject responds to a simple flashing-light presented in the LVF/RH with the right hand (Crossed condition) more slowly than in the LVF/RH with the left hand (Uncrossed condition) and vice versa. The reaction time between the Crossed and Uncrossed condition Difference is abbreviated as CUD. One study (Milner, Jeeves, Silver, Lines \& Wilson, 1985) tested a 20 year-old male with callosal agenesis whose intelligence was within normal range. They compared his CUD to a simple flashing-light with the normal CUD (i.e., 2-3 msec). The hypothesis was that if alternative pathways are available for individuals with callosal agenesis, this CUD should be longer than normal CUD because information transfer through the CC is assumed to be fastest for interhemispheric information transfer because it directly connects the two cerebral hemispheres. The results showed that this individual with 
callosal agenesis required a significantly longer CUD (mean $15.9 \mathrm{msec}$ ) than the normal CUD (2-3 mesc) to respond to the flashing-light presented to his LVF/RH with his right hand. This finding was consistent with a previous study (Reynolds \& Jeeves, 1974). The long CUD indicates that there is an alternative or indirect pathway available for individuals with callosal agenesis.

A question that arises is whether or not this alternative pathway is also available for use by commissurotomized patients (Tassinari, Aglioti, Pallini, Berlucchi, \& Rossi, 1994). Tassinari, et al. compared the CUD to a simple flashing-light presented in either the LVF/RH or RVF/LH among seven partially commissurotomized patients, a completely commissurotomized patient, and two individuals with callosal agenesis. In all the partially commissurotomized patients, the $\mathrm{CC}$ was sectioned from the anterior third to the fifth but the splenium was spared. The results showed that the partially commissurotomized patients exhibited the normal CUD (mean, $2.8 \mathrm{msec}$ ), whereas the completely commissurotomized patients showed the longest CUD (83.2 msec), and the callosal agenesis patients showed a CUD in the middle of the range (mean, $22.5 \mathrm{msec}$ ). The authors interpreted the findings such that the splenium can process visual information in partially commissurotomized patients; the much shorter CUD in the individuals with callosal agenesis suggested that the subcortical pathways available for individuals with agenesis were not available for completely commissurotomized patients. Thus, the authors concluded that subcortical pathways exist.

In summary, studies with commissurotomized patients have helped researchers in disclosing a callosal role in interhemispheric interaction. It appears that the $\mathrm{CC}$ has a role in visual and tactile information transfer between the two hemispheres. 
Commissurotomized patients demonstrated disconnection effects when they were asked to verbally identify an object presented in the LVF/RH. This was interpreted as a failure of information transfer from one hemisphere to the other. In contrast, individuals with callosal agenesis can compare stimuli presented in the bilateral visual fields, suggesting there are alternative subcortical pathways available for this population but not for commissurotomized patients.

Since the CC connects mainly homologous cortical areas in the two cerebral hemispheres, Preilowski $(1972,1975)$ postulated that the function of the CC must correspond to cortical functions. To test this hypothesis, he designed an experiment to investigate bilateral motor coordination with commissurotomized patients. The next section will discuss the role of the $\mathrm{CC}$ in motor coordination. As in the previous section, the discussion focuses on findings from the commissurotomized patients and individuals with callosal agenesis.

\subsubsection{Motor Coordination}

Preilowski (1972) hypothesized that the anterior function of the CC corresponds to the anterior function of the cortical areas. Specifically, he believed that the $\mathrm{CC}$ has an important role in bimanual motor coordination. To test this hypothesis, he compared three groups: (1) epileptic patients with anterior commissurotomy (26-year-old male, 28year-old female), (2) epileptic patients without commissurotomy (20-year-old male, 21year-old female), and (3) normal subjects (41-year-old male and female), using a crankhandle task (see Figure 5.4). The task required moving two handles, in which turning one registered as horizontal movement and turning the other registered as vertical 
displacement, in order to draw a line at certain angle. Thus, only with the left hand could a line be drawn at $90^{\circ}$, and only with the right hand could a line be drawn at $180^{\circ}$. To draw lines at $135^{\circ}$, both hands were required to perform identical parallel movements, and at $112.5^{\circ}$ and at $156.5^{\circ}$, the left or right hands would have to turn more than twice as fast as the other hand respectively.

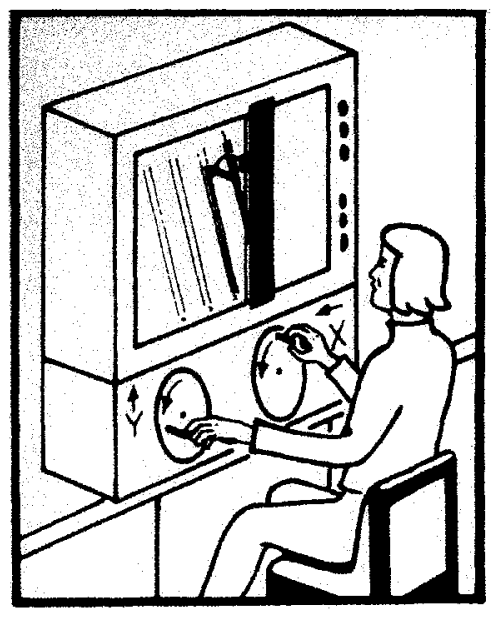

Apparatus

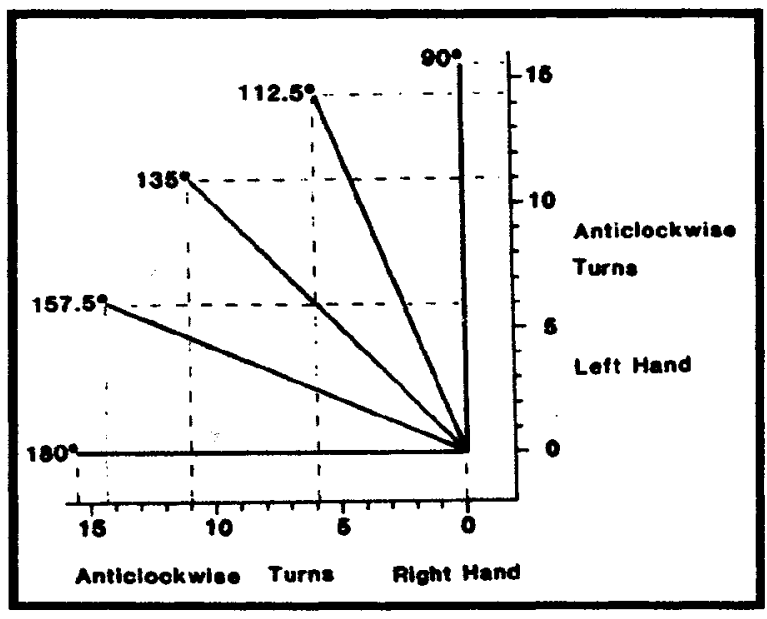

The task

Figure 5.4. Illustration of the crank-handles.

Illustration of how the subject turned two crank-handles in order to control a pen, which drew a line between guidelines on the screen, in front of the subject. To draw a line at $90^{\circ}$ only the left handle should be turned; to draw a line at $180^{\circ}$ only the right hand handle. To draw a line at $135^{\circ}$ both handles must be turned at the same speed. To draw a line at $157.5^{\circ}$ the right handle must be turned faster than the left and vice versa to draw $112.5^{\circ}$ (Preilowski, 1975).

While inspecting the progress of tracing the lines among the three groups, Preilowski noticed that at the initial stage, the groups did not differ; however, the normal subjects and the epileptic patients without commissurotomy learned more quickly than the commissurotomized patients, and their coordinated movements became streamlined even 
without visual feedback, whereas the commissurotomized patients never reached that point, even with viewing. Preilowski argued that a fast, smooth performance requires the exchange of motor information interhemispherically, especially in drawing a line without viewing, because visual cues are not available for drawing. He suggested that the partially commissurotomized patients lack such a control mechanism to coordinate bimanual movement because of sectioning of the $\mathrm{CC}$.

More importantly, the commissurotomized patients exhibited their worst performance in drawing a line at $112.5^{\circ}$, which requires turning the handle of the left hand/RH twice as fast as the right hand/LH. Preilowski interpreted this finding such that the right hand/LH must be inhibited (slowed down) while the left hand/RH turns the handle twice as fast as the right hand turns it, and this inhibition, controlled from the RH to the $\mathrm{LH}$ through the $\mathrm{CC}$, was lacking in the commissurotomized patients. Preilowski concluded that smooth bimanual motor coordination could be only achieved by inhibition through the anterior callosal connections, i.e., the midbody, in normal people. Subsequent studies (Milner \& Kolb, 1985; Preilowski, 1975) confirmed poor bimanual coordination in commissurotomized patients.

Bimanual motor coordination was also examined in two individuals with callosal agenesis (Jeeves \& Silver, 1988) using the crank-handle apparatus used in the studies by Preilowski $(1972,1975)$. These patients exhibited very slow performance even after ample practice compared to the controls. Like the commissurotomized patients, they could maintain the correct angle of the line with visual feedback but not without visual feedback. The findings showed that their performance was worst in drawing at $112.5^{\circ}$ 
without visual feedback. This indicates that the $\mathrm{CC}$ has an inhibition mechanism, which enables the two hands to act in a coordinated way during bimanual movements.

In summary, poor bimanual motor coordination was exhibited by commissurotomized patients as well as individuals with callosal agenesis. These studies suggest that the $\mathrm{CC}$ has an inhibitory role in motor coordination between the two hemispheres.

The traditional view of interhemispheric interaction holds that our cognitive functions can be achieved by hemispheric specialization, e.g., language for the LH and visuo-spatial abilities for the RH (Gordon \& Sperry, 1969; Sperry, 1968, 1974; Sperry et al., 1969). However, a new view, which emerged from studies with commissurotomized patients as well (Banich \& Karol, 1992; Hellige, 1993; Levy \& Trevarsen, 1976; Levy et al., 1972; Sperry, 1982), argued that the CC has a role of information integration as well as information transferor between the two hemispheres.

The next topic will be the callosal role in the integration of interhemispheric information.

\subsubsection{Integration}

An interesting phenomenon related to commissurotomy is that commissurotomized patients appear to be unable to consciously integrate information between the two hemispheres. When Sperry (1968) flashed an object to the LVF/RH and $\mathrm{RVF} / \mathrm{LH}$ of a commissurotomized patient, he noticed that the patient was unaware that the objects flashed to the LVF/RH and RVF/LH were identical. For example, when a picture of a woman's nude body was flashed to her LVF/RH, she started giggling. When 
asked what she saw, she answered "nothing but a flashing light," and she could not explain why she giggled. Apparently the patient was unable to consciously process the information from the RH to the LH but could unconsciously do so. Sergent (1990) also observed that in a face recognition task, commissurotomized patients did not realize that the same face was presented to both visual-fields, and thus, when the patients saw the same face in the RVF/LH after viewing it in the LVF/RH, they always stated that they had never seen the face before. Furthermore, Levy, et al. (1972) tested a commissurotomized patient using a chimeric figure, which is composed of two half-faces jointed at a midpoint (Figure 5.5). The patient was taught that one face was 'Dick' and the other was 'Bob'. When a chimeric picture consisting of Dick (right side) and Bob (left side) was briefly flashed to the patient, she always said it was 'Dick'. When she was asked if there was something odd about the picture, she denied it. This suggests that information about faces was not consciously integrated in this commissurotomized patient.

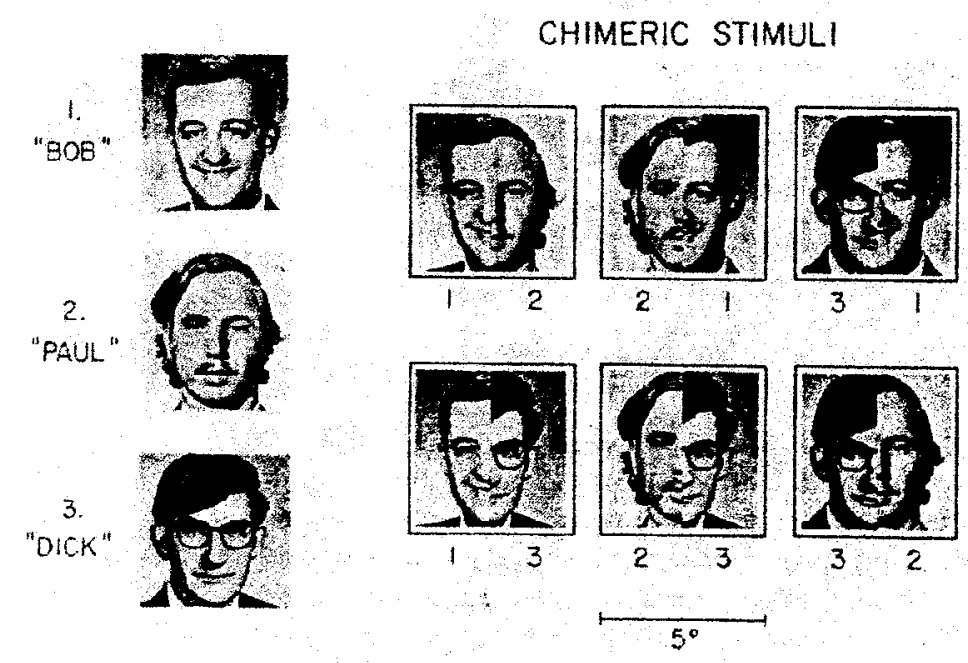

Figure 5.5. Test for recognition of faces.

(Levey, Trevarthen, \& Sperry, 1972). 
Sperry (1982) argued that the two hemispheres, although specialized for different cognitive functions, normally work together as a unit instead of working independently in order to achieve cognitive functions. Levy (1968) also proposed that the two hemispheres are designed qualitatively differently and have mutually antagonistic modes of cognitive processing; i.e., the LH is basically analytic and sequential, while the RH is spatial and synthetic, but there is a benefit to being able to use both functions together. If the two hemispheres work together, information in each hemisphere must be integrated, presumably through the $\mathrm{CC}$.

For example, Levy and Trevarsen (1976) found that the performance of commissurotomized patients did not always accord with the traditional view of hemispheric specialization. When a choice depicting two objects (shown in Figure 5.6) was briefly flashed to a commissurotomized patient in the center of the VF in open vision, the patient was asked to choose one picture from a list of pictures closely associated with the one flashed to them a moment before. There were three choices: (1) a LVF/RH selection (appearance), (2) a RVF/LH selection (function) or (3) a bilateral selection (LVF/function and RVF/appearance) (see Figure 5.6, 3-2 in chimeric stimuli). For example, in the case of a combination of a cake (left side) and scissors (right side), if a hat were chosen, the decision was made by the RH (appearance); if thread were chosen, the decision was made by the LH (function); if a spoon and fork were chosen, the decision was made by bilateral function, which is incongruent with hemispheric specialization. Surprisingly, the patients chose the bilateral selection as often as the LVF and RVF selections in the chimeric picture test. Levy and Trevarsen introduced the term 'metacontrol' for this phenomenon: they argued that each hemisphere did not always 
perform based on its specialization when both hemispheres were given an equal chance to select the picture (e.g., bilateral selection). They concluded that our cognitive behaviours are not always controlled only by hemispheric specialization but also by metacontrol systems.

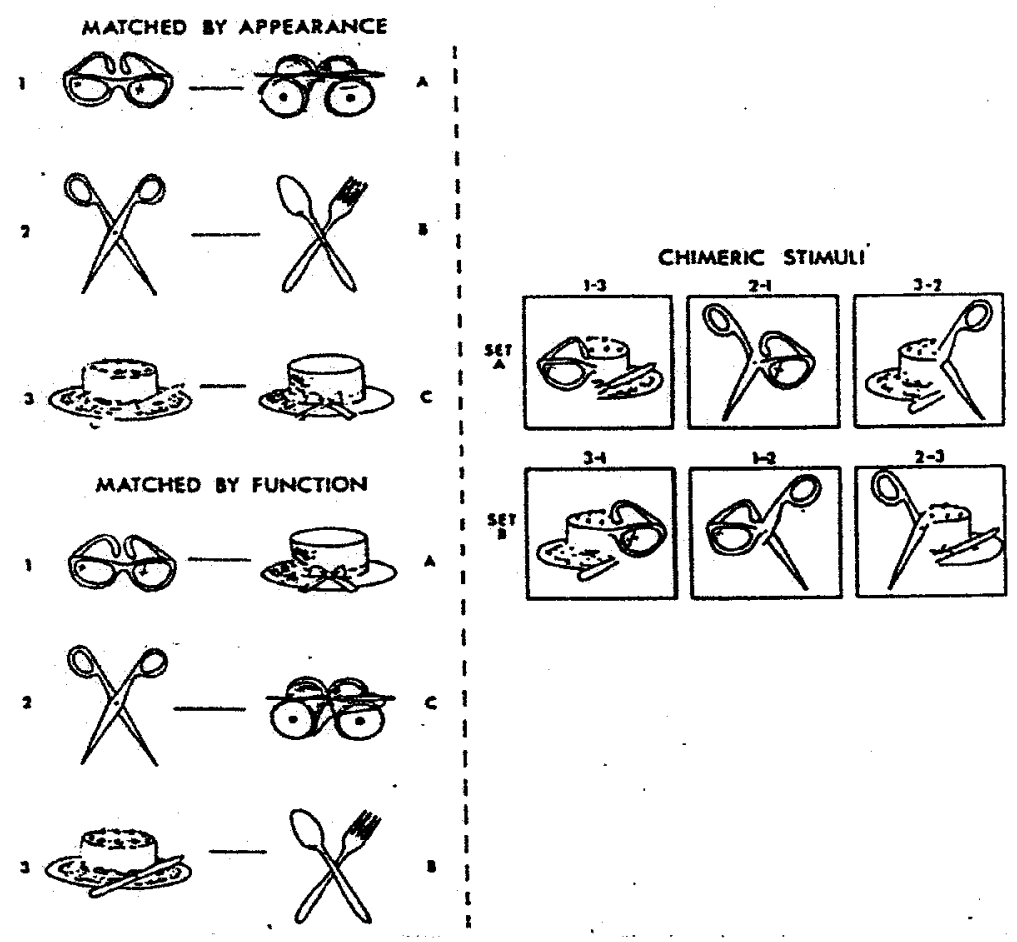

Figure 5.6. Appearance-Function matching chimeric pictures test (Levy \& Trevarthen, 1976).

Proposed metacontrol mechanisms were further investigated by Hellige, Taylor, and Eng (1989) with normal subjects using a tachistoscopic technique. They presented stimuli consisting of a consonant-vowel-consonant (CVC) nonsense syllable in different viewing conditions, i.e., LVF, RVF, and bilateral VFs. The subjects were asked to pronounce and spell the CVC syllable in each trial. The findings demonstrated overall 
superior performance in the RVF/LH as expected, confirming the traditional view that the $\mathrm{LH}$ is superior for processing linguistic stimuli. More importantly, an error analysis revealed that the error pattern on bilateral trials was more similar to errors made on LVF/RH trials than on RVF/LH trials. That is, errors in the last syllable of CVC letter strings were made more often on LVF/RH and bilateral trials than on RVF/LH trials. The authors argued that this result did not accord with their expectation that the error patterns of the bilateral trials would be similar to those on the RVF/LH trials because the LH was supposedly responsible for phonetic processing. Since the data showed that the overall error percentage on the bilateral trials was lower than on the RVF/LH and LVF/RH trials, they suggested that the brain might spontaneously engage in the mode of processing previously associated with superior performance (i.e., learning) rather than automatically utilizing hemispheric specialization.

In contrast to commissurotomized patients, acallosal individuals have no difficulty in comparing digits and letters presented bilaterally by visual half-field presentation; even six-letter words (e.g., cotton, hasten) presented with the first three letters in the LVF/RH (cot) and the rest of the letters in the RVF/LH (ton), are processed as a whole and not as two discrete words (e.g., cot and ton, has and ten) (Corballis \& Finlay, 2000). These results suggest that unity of consciousness in visual experiences exists in the acallosal population. This agreed with previous evidence that individuals with callosal agenesis do not show striking disconnection. The implications are two-fold: (1) the CC does not have any role in the integration of conscious experience in this population (Sauerwein \& Lassonde, 1994), and (2) the plasticity of the cerebral 
hemispheres in terms of developing alternative pathways to communicate between the two hemispheres (Tassinari et al. 1994).

Why would it be advantageous to integrate information between the two hemispheres? This issue was explored by Banich and colleagues in a series of studies using a variety of stimuli, including numbers, letters, and geometric shapes (e.g., Banich \& Belger, 1990; Banich \& Karol, 1992). Banich and colleagues concluded that bilateral processing may enhance efficiency when a task becomes complex. For example, when subjects had to simply decide if the target matched one of the probes in physical form (e.g., A and A or 2 and 2), speed and accuracy decisions were superior in the unilateral presentation condition compared to the bilateral presentation condition; however, when more complex decisions were involved in the response, such as whether items have a similar name (e.g., A and a) or add up to 10 or more (e.g., 2 and 9), the bilateral presentation showed an advantage. This suggests that unilateral processing is advantageous in simple tasks, and as task demand increases, the two hemispheres cooperate to enhance efficiency. In another example, Belger and Banich (1992) compared a physical-identity match task and a name-identity match task. In the physical identity match task, subjects were asked to indicate whether the bottom letter matched either of the top letters (e.g., A-B \& A). In the naming-identity match task, the subjects were asked to do the same task as the physical-identity match task; however, the case of the letters was manipulated (e.g., A-B \& a). Thus, processing demands were greater in the naming-identity match task than in the physical-identity task. The results showed that the bilateral presentation was responded to faster than the unilateral presentation in the naming-identity task, and the opposite pattern was found in the physical-identity task. 
Although studies with commissurotomized patients have contributed to establishing the view that the $\mathrm{CC}$ has a role in the integration of interhemispheric information into a unified consciousness, there is still controversy surrounding this issue.

In summary, in contrast to the traditional view of the role of $\mathrm{CC}$, a new view is proposed that the role of the $\mathrm{CC}$ is to integrate and unite information in addition to information transfer between the two hemispheres. This was demonstrated in a chimeric picture test, where commissurotomized patients specifically exhibited unexpected task performances, a phenomenon referred to as metacontrol. Normal subjects also demonstrated metacontrol mechanisms in the CVC nonsense reading experimental task. This suggests that our cognitive behaviours are not always controlled by unilateral processing based on hemispheric specialization, but also by bilateral processing based on integration of information in a unified conscious mind. The advantage of the integration of interhemispheric information may be to enhance the efficiency of information processing.

In terms of language, both commissurotomized patients and individuals with callosal agenesis do not demonstrate obvious deficits. Sectioning and absence of the $\mathrm{CC}$ appear not to have any affect on language. However, children with callosal agenesis, who have normal intelligence, have demonstrated some subtle deficits in language processing. The next section will discuss the role of the $\mathrm{CC}$ in language and reading abilities with children with callosal agenesis, focusing on their reading ability. This section is relevant to the discussion of developmental dyslexia in the present thesis. 


\subsubsection{Language}

Contrary to reports of preserved language in children with callosal agenesis, some researchers have reported that children with callosal agenesis who had average or above average IQs showed subtle deficits in language characterized by phonological processing, complex syntactic knowledge (e.g., passive negative sentences), and pragmatic (tacit knowledge) components (Dennis, 1981; Jeeves \& Temple, 1987; Temple, Jeeves, \& Vilarroya, 1989, 1990). However, even though these children had difficulty in processing phonology, they were not dyslexic.

Temple et al. (1990) tested two children with callosal agenesis (age of 9.4 years and 13.2 years). When a standardized reading test was administered (Schonell \& Goodacre, 1971), it revealed that they had an age equivalent reading age (9.1 years and 13.4 years). Their tested IQs were 104 and 105 on the Weschler Intelligence Scale for Children-Revised (WISC-R) (Wechsler, 1981). However, these children had different reading patterns from normal readers at their age. That is, they read words far better than nonwords, which is called the lexical effect (Adams, 1990). Normally, young children become able to read nonwords along with regular words when they have learned the phonological rules. Thus, the lexical effect disappears when letter-sound rules are acquired (Temple \& Ilsley, 1994). Temple et al. (1990) argued that the acallosal children in their sample demonstrated evidence of good orthographic but poor phonological knowledge. The authors suggested that the children's reading ability relied mainly on lexical analogy rather than on phonological rules. This tendency is reflected in fewer errors by applying GPC rules in reading irregular words (e.g., island-> island, come- 
$>$ kome) and good performance in reading homophonic nonwords (that sound like a real word) when the nonwords were visually similar to words (e.g., brane-brain). Temple, et al. speculated that traditionally the LH has been considered as the responsible hemisphere for phonological processing, and thus, given a normal $\mathrm{LH}$, it is not immediately obvious why the absence of the CC should adversely affect phonological processing in this population. They further argued that the $\mathrm{CC}$ may be involved in developing the LH specialization for reading and subsequently in the fine tuning of linguistic processing. The study clearly suggested that the $\mathrm{CC}$ was essential for the development of explicit phonological processing skills and the normal phonological reading route (refer to Chapter 2).

In summary, children with callosal agenesis demonstrated poor phonological processing, although their reading was apparently normal. A detailed analysis of their reading pattern revealed, however, their reading relied on analogical strategies evidenced by the persistence of the lexical effect in reading and they showed fewer errors by applying GPC rules in reading irregular words. Thus, subtle differences in reading are observed when the $\mathrm{CC}$ is absent from the brain.

Lateralization in language (Galaburda et al., 1985; Pugh, Mencl, Jenner et al., 2000 ) is stronger for right-handed than left-handed people (Bryden, 1982; Beaton, 1985). Many studies have suggested that females have bilateral language representation (e.g., Shaywitz et al., 1995; Witelson, 1989), although this hypothesis is still controversial (Beaton, 1985). Since the CC is the main fiber tract connecting the two hemispheres, functional laterality, handedness, and gender may have some connection with the size of the CC. This literature is discussed in the final section of this chapter. 


\subsection{Callosal Size, Gender, and Handedness}

The first post-mortem study (De Lacoste-Utamsing \& Holloway, 1982) of the CC found sexual dimorphism in this structure, i.e., females have a more bulbous and wider splenium (posterior fifth) than males, and the absolute splenial surface area is larger in females than males. Holloway and De Lacoste-Utamsing (1986) replicated these findings, adding that the total $\mathrm{CC}$ area is absolutely larger in females than males despite the fact that females normally have a smaller brain size than males. The authors reasoned that a large callosal area indicates more capacity for communication than a small callosal area. They also suggested that female brains are less lateralized in cognitive functions than male brains since they have larger corpus callosa. They further argued that male brains are more specialized for cognitive functions, such as language for the $\mathrm{LH}$, than are female brains. The majority of follow-up studies, however, failed to replicate these results (Aboitiz et al., 1992; Byne, Bleier \& Houston, 1988; Clarke, Kraftsik, Van Der Loos, \& Innocenti, 1989; Clarke \& Zaidel, 1994; Habib, Gayraud, Oliva et al., 1991; Jancke, Wunderlich, Schlaug, \& Steinmetz, 1997; Kertesz, Polk, Howell, \& Black, 1987; Oppenheim et al., 1989; Pozzili, Bastianello, Bozzao et al., 1994; Weis, Weber, Wenger, \& Kimbacher, 1989; Witelson, 1985, 1989).

For example, Witelson found no gender difference in total callosal size in two post-mortem studies $(1985,1989)$. More importantly, her studies found significant hand preference effects, i.e., non-consistent right handers (nCRH) had a larger total callosal area than consistent right handers (CRH). In her study, the CRH were defined as those who reported a right hand preference from more than nine out of 12 handedness tasks 
(75\%) of a modified version of Annett's handedness questionnaire (1967) (e.g., tooth brushing, threading a needle). The nCRH were those who did not meet criteria for the $\mathrm{CRH}$; including those who reported a consistent left-hand preference in the handedness task. For this post-mortem examination project, Witelson obtained consent forms from 93 cancer patients prior to death; these patients then completed psychological tests including this questionnaire. Her study (1989) also found that the nCRH males had a larger isthmus than the CRH males, whereas neither the CRH nor nCRH females showed this sexual dimorphism. Witelson (1989) argued that the difference between CRH and $\mathrm{nCRH}$ in males supports the hypothesis that callosal variation is related to language lateralization in males. She reasoned that since the isthmus connects the PT area (Wernicke's area) with the homologous area in the RH (De Lacoste et al., 1985), large callosal size in the nCRH males indicated more interhemispheric interaction than small callosal size in the CRH males in language processing. She further suggested that the larger isthmus in females than males and no callosal size difference between the CRH and $\mathrm{nCRH}$ in females implies that females generally have more interhemispheric interaction.

In support of this view, an fMRI study (Shaywitz et al., 1995), sample of normal female brains (19 right-handed women, mean age of 23.0 years), showed bilateral activation in Broca's area during a rhyming judgment task, whereas normal male brains (19 right-handed men, mean age 28.0 years) showed activation lateralized to the left inferior frontal regions including Broca's area during the same task. The bilateral activation in the females in this sample implies the participation of the $\mathrm{RH}$ in phonological processing at least in some females (see Figure 5.7). Another post-mortem 
study (Aboitiz et al., 1992) revealed that there was a negative correlation between isthmus size and the absolute value of the planum temporale asymmetries. Since the isthmus connects Wernicke's area to the homologous area in the $\mathrm{RH}$, this finding implies that lesser callosal connections mean more asymmetries in the PT areas. The significant correlation was found only in males $(r=-0.55)$ and not in females $(r=-0.14)$. These findings support the concept that male brains are more lateralized than female brains in language and suggests that callosal size and laterality are in an inverse relationship (Beaton, 1985; Witelson, 1985, 1989).

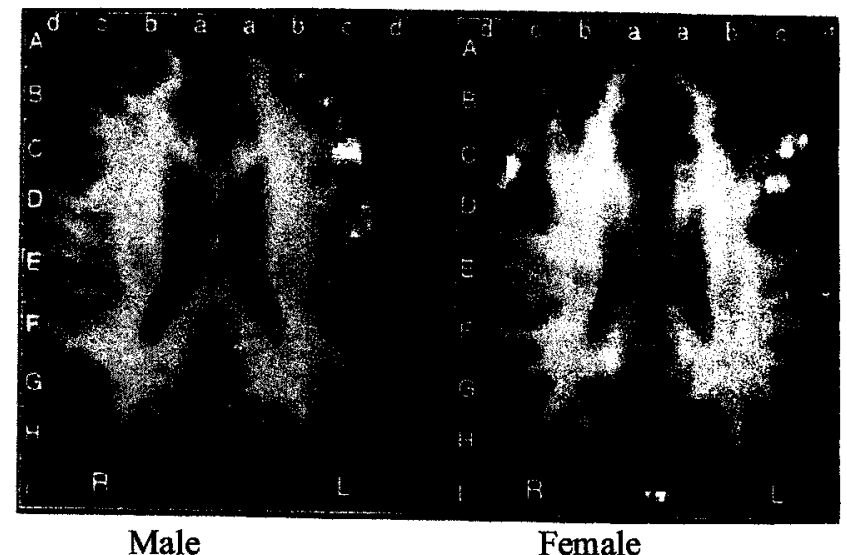

Figure 5.7. Composite fMRI images of the distribution of activation in a rhyming judgment task. In the task (phonological processing) 19 males (left image) were compared to 19 females (right image) (Shaywitz et al., 1995).

Clarke and Zaidel (1994) found a positive correlation between the size of the isthmus (Wernicke's area) and degree of handedness in a sample of normal adult readers in a primed lexical decision task (LDT). An LDT is a task in which a participant makes a judgment as to whether a presented letter string is a word or not. A priming paradigm 
measures the effect of semantic relatedness between a prime and a target word. For example, when two paired words (cat-dog vs. pen-bus) are successively presented, the semantically related target word (dog) can be recognized faster than the semantically unrelated target word (bus). The spreading activation theory (e.g., Anderson, 1976; Collins \& Quillian, 1969; Collins \& Loftus, 1975; Neely, 1977) proposed the following assumption: associatively/semantically related words (e.g., cat-dog) are more closely linked than unrelated words (e.g., pen-bus) in the mental lexicon, and thus, when a priming word (cat) is activated, the activation spreads to the target (DOG) because of associated/semantic relatedness. Thus, the spreading activation reduces the time required for activation in the related word (dog), and facilitates the response to the target. However, this facilitation effect cannot be expected for unrelated word pairs (PEN-BUS) because these words are not strongly linked in the mental lexicon. It logically follows that in a primed LDT, when the target is associated with the prime (e.g., doctor-nurse), the target (nurse) is recognized faster and more accurately recognized as a word than an unrelated word target (hand) in an unrelated pair (e.g., desk-hand). Using the primed LDT paradigm, Clarke and Zaidel found that the right-handed males in a normal sample demonstrated larger priming effects in the RVF/LH than in the LVF/RH, but these effects were not found in the left-handed males. This suggests that semantic processing is lateralized to the $\mathrm{LH}$ in right-handed males; however, the left-handed males seemed to use both hemispheres in this semantic task. This finding was consistent with a previous study (Habib et al., 1991). In contrast, several MRI studies (Kertesz et al., 1987; O’Kusky, Strauss, Kosaka et al., 1988; Yoshii, Barker, Apicella et al., 1986, Jancke et al., 1997) failed to find handedness and gender effects. 
No consensus has yet been established in terms of the relationship between callosal size, gender, and handedness. Clarke et al. (1989) commented that the reason for the inconsistent results was a lack of large sample sizes, which would be imperative considering the large variability in individual callosal sizes and shapes, and inconsistent parameters among studies, such as callosal thickness, length and partitioning of the CC as discussed previously (see Figure 5.1).

Controversy still exists around callosal size associated with anatomical asymmetry and behavioural-functional asymmetry. There is as yet no definitive answer as to whether the brain with a small $\mathrm{CC}$ is more lateralized than the brain with a large $\mathrm{CC}$ in cognitive functions.

In summary, despite early findings suggesting that females have larger corpus callosa than males, most consequent studies found no gender differences in callosal size. Surprisingly, despite its massive size and central location in the brain and over a century's research, the roles of the CC has not been yet established. However, studies with commissurotomized patients and children with callosal agenesis have provided evidence that the $\mathrm{CC}$ is involved in diverse human cognitive functions including visual and tactile information processing, bimanual motor coordination, and integrating information to form a unified consciousness. In the language domain, although callosal sectioning did not cause aphasia and alexia, children with callosal agenesis demonstrated poor phonological processing, despite the fact that they had relatively good reading ability (Dennis, 1981; Temple, 1987; Temple et al., 1989, 1990). This implies that the $\mathrm{CC}$ is essential in developing phonological processing but is not essential for reading. Regarding the size of the $\mathrm{CC}$, some studies found that females have larger corpus callosa 
than males. Witelson $(1985,1989)$ found a correlation between callosal size and handedness. Males with $\mathrm{CRH}$ showed much smaller callosal connection than males with $\mathrm{nCRH}$, whereas females did not show any difference between $\mathrm{CRH}$ and $\mathrm{nCRH}$. This is consistent with evidence that males with $\mathrm{CRH}$ are more lateralized than females and males with $\mathrm{nCRH}$. Other studies found that callosal size is relative to brain size regardless of gender. Controversy still exists surrounding this issue.

The next chapter will discuss an interhemispheric disconnection theory based on the view that deficits in interhemispheric interaction are involved in developmental dyslexia. 


\section{Chapter 6: The interhemispheric disconnection theory}

The interhemispheric disconnection theory holds that $\mathrm{DD}$ is primarily caused by deficits in interhemispheric interaction (Badian \& Wolff, 1977; Fabbro, Pesenti, Facoetti et al., 2001; Gross-Glenn \& Rothernbero ～, 1984; Gross, Rothernbero, \& Schottenfeld, \& Drake, 1978; Gladstone \& Best, 1985; Gladstone, et al, 1989; Hynd, Hall, Novey et al., 1995; Larsen, Hoien, \& Odegaard, 1992; Quinn \& Geffen, 1986; Rousselle \& Wolff, 1991; Summerfield \& Michie, 1993; Yeni-Komshian, Isenberg, \& Colberg, 1975). The theory assumes that DD is attributable to impairments in interhemispheric interaction through the corpus callosum $(\mathrm{CC})$ rather than dysfunction confined to the left hemisphere. The association between DD and interhemispheric interaction deficits was observed by Yeni-Komshian et al. (1975) in a visual half-field experiment and Badian and Wolff (1977) in a motor sequencing study. The view was then further elaborated by Gladstone and Best in their review article (1985). This theory is not in the current mainstream compared to the phonological deficit theory and the left hemisphere dysfunction theory (see discussion in Chapters 2 and 3). It is, however, supported by ample behavioural studies, such as bimanual motor coordination studies (Fabbro et al., 2001; Wolff, Cohen, \& Drake, 1984; Wennekes \& Njiokoktjien, 1991) and sensory perception studies (Gross-Gelnn \& Rothenberg, 1984; Summerfield \& Michie, 1993). It is also supported by physiological studies from autopsy studies (Drake, 1968; Witelson, 1985), MRI studies (Robichon \& Habib, 1998). The implication of these studies is that $\mathrm{DD}$ is associated with structural abnormalities involving the $\mathrm{CC}$ and may well be caused by deficits in interhemispheric interaction. 


\subsection{Evidence for the Theory}

As discussed in Chapter 5, early studies with commissurotomized adult patients (Preilowski, 1972, 1975) revealed impairments in bimanual but not in unimanual motor coordination. Poor bimanual motor coordination was also exhibited by children with callosal agenesis (Chiarello, 1980; Jeeves \& Silver, 1988; Silver \& Jeeves, 1994). The findings of these studies suggest that interhemispheric interaction through the $\mathrm{CC}$ is necessary to coordinate bimanual motor movement and may also be needed for phonological processing. Furthermore, children with callosal agenesis but not commissurotomized adult patients demonstrated poor phonological processing (Temple \& Ilsley, 1994; Temple et al., 1989). Interestingly, poor phonological processing is the major deficit in DD children. This suggests that the $\mathrm{CC}$ requires the development of phonological skills, but once these skills are acquired, the CC may be not necessary for phonological processing.

In terms of bimanual motor coordination, DD children often show poor performance, especially in the left hand (Badian \& Wolff, 1977; Klicpera \& Wolff, 1981; Wolff et al., 1984, Wolff, Michel, \& Drake, 1990). Since the left hemisphere is believed to control temporal processing in motor movements, the left-hand deficit in bimanual motor coordination may be attributable to LH dysfunction (Rudel, 1985). Thus, poor bimanual coordination in DD seems to support the left hemisphere dysfunction theory. However, the left-hand deficits appear to be manifested only when a motor coordination task involves the bimanual alternating mode. The deficit is not evident in the unimanual mode. For example, Wolff et al.'s study (1990) tested DD subjects (mean age of 13-18 
years, five boys and five girls) using a tapping task, in which the participants were asked to tap two mechanical keys placed eight inches apart alternating between their left and right hands in time to the beat of a metronome (alternation trials) or to tap with the left or right hand alone (single hand trials). Their motor deficit was evident only when the task required asynchronous finger movement in the alternation trials. It was not evident when the task required synchronous finger movement in both hands and in the single hand trials. The authors suggested that the deficiency in bimanual motor coordination observed in this population may be due to a disturbance in interhemispheric cooperation.

Gladstone et al. (1989) also investigated bimanual motor coordination in DD children. In their study, the DD children (18 boys, mean age of 12.08 years) were asked to follow a line on a screen using a plotter pen controlled by two small, remote handmanipulated potentiometers, similar to the "Etch-A-Sketch" toy. Following six different lines requires a variety of independent hand movements in terms of speed and direction. In order to move the pen in different directions, the two knobs must be used differently. For example, to follow a straight line that deviates at $26.5^{\circ}$ horizontally, the bimanual hand ratio was set at 2:1 for the left and right hands respectively in a clockwise direction (unidirectional condition). However, to follow a straight line at $153.5^{\circ}$, the hand ratio must be 1:2 with one hand moving in a clockwise and the other moving in a counterclockwise direction (bidirectional condition). The completion time was measured under two conditions, visual feedback (free viewing) and non-visual feedback. In the latter condition, halfway through the trial, the subject's view was blocked and he or she was instructed to complete the line based on memory. After the completion of the non- 
visual feedback condition, the effect of visual memory on line orientation was assessed. In the trial, all subjects were asked to reproduce the six angles from memory. The results showed that in both the visual feedback and non-visual feedback conditions, the DD children were significantly slower and less accurate in the bidirectional trials than the controls. A striking finding was that nearly all the dyslexic children, but none of the controls, unknowingly reversed the directions from bidirectional to unidirectional movements of the two hands when visual feedback was removed. Gladstone et al. argued that the DD children had difficulty in maintaining the bidirectional movements in this task, and observed greater left- than right-hand impairment in this condition. This is coincident with the findings in commissurotomized patients of the crank-handle task (see Chapter 5.3.2) in bimanual coordination (Preilowski, 1972, 1975). Gladstone et al. argued that the impairment in the bidirectional movement in the DD children demonstrated dysfunction in interhemispheric interaction because they could not coordinate the two hands. The authors suggested that this might be due to the absence of callosal mediation and might influence the transfer of motor control from the left hemisphere to the right hemisphere (left-hand deficit).

Summerfield and Michie (1993) examined the efficiency of callosal transfer in the sensory modality in a sample of $16 \mathrm{DD}$ boys (mean age of 9.0 years) using a finger localization task. In this task, the participants were required to locate a stimulated finger by touching it with the thumb on the same hand (Uncrossed condition) or using the thumb of the opposite hand to identify the corresponding stimulated finger (Crossed condition). The DD children were compared with a chronological age-matched group and a reading age-matched group (mean age of 7.0 years). The finding demonstrated that the 
DD children performed significantly more poorly than their two-years-younger control group on the Crossed Condition. However, the chronological age-matched control group showed better performance than the reading age-matched control group. This demonstrated that accuracy in the finger localization task on the Crossed Condition improved with age in the normal course of development. This was consistent with a previous study (Quinn \& Geffen, 1986). More importantly, the task performance on the Crossed Condition was significantly correlated with the standardized reading scores. Thus, the authors concluded that efficiency in interhemispheric information transfer relies on callosal development and that the poor performance in DD children on the Crossed Condition is due to a dysfunction of the CC. This conclusion is compatible with previous studies (Gross-Glenn \& Rothenberg, 1984; Gross et al., 1978).

MRI studies (Duara et al., 1991; Hynd et al., 1995; Robichon et al., 2000; Robichon \& Habib, 1998; Rumsey, Casanova, Mannheim et al., 1996) have provided further evidence showing morphological abnormalities of the $\mathrm{CC}$ in developmental dyslexia, although there is some contradictory evidence among the findings. For example, Hynd et al. (1995) found a smaller genu (see Figure 5.2) in dyslexic adults than in controls but no group differences for the splenium (see Figure 5.2). In contrast, Duara et al. (1991) found an opposite pattern of abnormality: a larger splenium but no difference in the genu was observed in their female dyslexic patients. Rumsey et al. (1996) found that the posterior third (isthmus and splenium) of the CC was larger in the adult dyslexics than normal reader controls. Robichon and Habib (1998) found that, overall, adult dyslexics have a thicker, larger, and more round isthmus than normal controls. One study failed to find significant morphological difference in the $\mathrm{CC}$ between 
dyslexics and normal controls (Pennington et al., 1999). Although the findings are inconsistent, the positive MRI studies are persuasive and suggest that DD adults have some impairment in the $\mathrm{CC}$.

In summary, DD children demonstrated poor bimanual motor coordination and poor performance on crossed finger localization tasks. MRI studies with adults have also demonstrated some abnormalities in both the anterior and posterior regions of the $\mathrm{CC}$.

\subsection{Summary}

The interhemispheric disconnection theory holds that $\mathrm{DD}$ is attributable to a lack of efficient communication between the two cerebral hemispheres. This theory explains the deficits in bimanual motor coordination observed in DD children (Badian \& Wolff, 1977; Klicpera \& Wolff, 1981; Wolff et al., 1984; Wolff et al., 1990; Gladstone et al., 1989; Fabbro et al., 2001) and callosal transfer deficits observed in tactile information processing (Quinn \& Geffen, 1986; Summerfield \& Michie, 1993). In these tasks, dyslexic participants performed much more poorly in bimanual than in unimanual conditions. This suggests interhemispheric interaction deficits in this population. Furthermore, there are strong similarities in the performance of motor coordination and phonological processing between dyslexics and patients with callosal impairments. Poor bimanual motor coordination was seen in commissurotomized patients (Preilowski, 1972, 1975) and children with callosal agenesis (Chiarello, 1980; Silver \& Jeeves, 1994). Acallosal children but not commissurotomized patients demonstrated poor phonological processing (Temple \& Ilsley, 1994; Temple et al., 1989), even though they are not dyslexic. A positive correlation between the standardized reading scores and the 
bimanual tactile task on the Crossed Condition was also found in Summerfield and Michie's study (1993). In addition, MRI studies suggest that dyslexic adults have some morphological abnormalities in the $\mathrm{CC}$, such as thinning of the $\mathrm{CC}$ and the smaller genu or the larger isthmus, compared to normal controls.

\subsection{Discussion}

Poor phonological processing demonstrated by acallosal children is, in the present context, a strongly suggestive association between developmental dyslexia and the $\mathrm{CC}$ (Temple \& Ilsley, 1994, Temple at al., 1989). Although acallosal children have adequate reading ability, their reading largely relies on sight vocabulary (Temple et al., 1989) rather than phonological skills. Since phonological processing is believed to be processed with the LH in most normal adults, it is not immediately clear why children with callosal agenesis show poor phonological processing. However, alexia research in the Japanese language (Sugishita et al., 1992) may shed some light on this issue (see Chapter 3.1). They suggested that low level of visual inputs from the $\mathrm{RH}$ to the $\mathrm{LH}$ through the splenium might be required for reading because pure alexia always accompanied right hemianopsia (blindness in one half of the visual field) among their Japanese patients. The authors suggest that pure alexia might result from a disconnection between the right visual cortex of the occipital lobes and the language-dominant LH. Thus, this study supports the view that the $\mathrm{CC}$ is necessary to transfer visual information from the $\mathrm{RH}$ to the LH for phonological processing in Japanese.

The poor bimanual coordination demonstrated by commissurotomized patients and children with callosal agenesis adds evidence to the theory of callosal deficit in DD. 
In spite of some inconsistent findings, MRI evidence has also demonstrated some abnormalities of the $\mathrm{CC}$ in adult dyslexics.

A question arises as to whether these abnormalities are necessarily associated with a specific reading disability or simply present in general poor reading ability. Although a study by Summerfield and Michie (1993) showed a positive correlation between the standardized reading scores and the bimanual tactile task, this study needs to be replicated in order to better understand the connection between interhemispheric interaction and DD. More direct evidence is also needed, such as a correlation between the size of the $\mathrm{CC}$ and reading ability in the normal population.

The interhemispheric disconnection theory is distinctive in that it stresses the relationship between two hemispheres rather than focusing on one hemisphere. Since the $\mathrm{CC}$ is involved in multiple brain functions, such as memory, attention, consciousness, and integration of information (refer to Chapter 5.3.3), the theory is promising in terms of exploring possibilities of the involvement of multiple brain systems in reading, that can account for the diverse characteristics and possible sub-types of developmental dyslexia. Before pursuing this topic, however, the four theories that have been presented here will be compared and evaluated in the next chapter. 


\section{Chapter 7: Evaluation of the four main theories}

As previously noted, developmental dyslexia (DD) is commonly defined as a specific reading disability that cannot yet be accounted for by low IQ, physical/mental disorders or environmental factors. Though genetic factors seem to contribute to DD in some cases (Oppenheim et al., 1989), the etiology is uncertain. It is also not clear whether one single factor or deficit can account for all the symptoms displayed by DD adults and children. Although the major deficit involved in DD is poor phonological processing (Hulme \& Snowling, 1992; Snowling, 1995; Sprenger-Charolles et al., 2000), some DD children are able to process phonology at an age appropriate level and instead show difficulty in lexical processing (Castles \& Coltheart, 1993; Coltheart et al., 1983; Ellis, 1994; Temple \& Marshall, 1983). This suggests that sub-types may exist (Castle \& Coltheart, 1993). Many DD children have minor cognitive problems, such as poor bimanual coordination (Gladstone et al., 1989; Summerfield, \& Michie, 1993), verbal memory (Kramer et al., 1999), sequential processing (Bakker, 1973), or visuo-spatial processing (Boden, \& Brodeur, 1999). In addition, some DD children have problems involving one element of the visual system, called the magnocellular system, and some do not (Best \& Demb, 1999). There is also anatomical variability in DD adults and children: some have abnormal and some have normal asymmetrical brain organization (Galaburda et al., 1985; Rumsey et al., 1997). Furthermore, two recent $\mathrm{fMRI}$ studies confirmed that DD adults and children have abnormal brain activation when performing a phonological processing task, 
but these activation patterns differed in DD adults and children (Pugh et al., 2000; Shaywitz, 2001). It is not clear why there are such diverse symptoms associated with DD.

With respect to developing a theory of developmental dyslexia, the diverse characteristics of DD need to be taken into account. However, each of the theories discussed previously focused on one specific characteristic of DD. As a result, each theory can explain some characteristics of DD but leaves out the rest. An integrated theory should account for all the characteristics seen in developmental dyslexia. This chapter will evaluate the four theories based on the accountability of the main characteristics of developmental dyslexia. These are: (1) poor phonological processing, (2) sub-types of developmental dyslexia, (3) visual problems, such as transporting letters in a word, (4) poor bimanual coordination, (5) minor cognitive problems, such as sequential processing (reciting numbers or the names of months), and (6) genetic predisposition.

The phonological deficit theory views dyslexia strictly within the linguistic domain. The linguistic deficit is specifically demonstrated in poor phonological processing, which is manifested in poor phonological awareness, poor lexical retrieval, and poor verbal working memory (WM) (Wagner \& Torgesen, 1987). The most dramatic manifestation of poor phonological processing is in reading nonwords (Rack et al., 1992). This is because DD children are insensitive to the segmentation of a word, resulting in difficulty in learning grapheme-phoneme conversion (GPC) rules, which is necessary to read nonwords. Thus, the phonological deficit theory claims that DD stems from linguistic deficits.

Since phonological deficits in DD are supported by ample evidence including brain studies, this theory reflects the current consensus in the dyslexic literature. However, the 
theory leaves out many characteristics of DD. There is little discussion of the causality of the linguistic deficits, including genetic factors. Furthermore, since the theory focuses only on deficits in phonological processing and not in lexical processing, it dismisses sub-types of DD (Castles \& Coltheart, 1993; Coltheart et al., 1983; Ellis, 1994). Neither subtle cognitive impairment nor poor bimanual coordination is discussed in the theory. Since the theory focuses on linguistic aspects, it could explore the possibility of integrating the linguistic dysfunction in the LH into this theory. In fact, recent functional neuroimaging studies (Milne et al., 2002; Pugh et al., 2000; Shaywitz et al., 2002) have provided support for the phonological deficit theory. The theory should make efforts to integrate anatomy, brain functions and linguistic deficits.

The left hemisphere dysfunction theory assumes that dyslexia is caused by structural and functional abnormalities in brain organization. The structural abnormalities are characterized by ectopic cells and abnormal asymmetry in the left PT area (symmetrical or reversed asymmetrical PT). The functional abnormalities are demonstrated by less activation in DD individuals than in normal individuals in the left posterior temporal regions. These regions are closely associated with reading systems. The contribution of this theory is to explain the causality of DD, including genetic factors, based on anatomical and functional evidence associated with brain specialization. The theory provides a good explanation of the sub-types of phonological and surface dyslexia based on the two major reading networks in the LH (Pugh et al., 2000). The structural and functional evidence fits well with a cognitive reading model (a dual route model) (Baron, 1979; Coltheart et al., 1993). It also accommodates a genetic predisposition to DD in relation to the development 
of the cerebral hemispheres, even though the precise determinant of cerebral asymmetries in developing human brains is not clear (Schachter, 1993).

This theory was developed based on the cerebral specialization approach, in which the LH is believed to be highly specialized for language. Galaburda (1993) argued that the presence of abnormal cells in the LH and abnormal organization in PT areas (symmetrical or reversed asymmetry) causes dyslexia. However, the theory fails to explain why the $30 \%$ of the normal population that have symmetrical or reversed asymmetrical PT areas are not dyslexic. Furthermore, there is no explanation as to why some DD children show normal leftward asymmetry. A recent MRI study (Rumsey et al., 1997) revealed that 16 adult righthanded dyslexic males had normal asymmetry in the PT. This is robust evidence that not all individuals with dyslexia have abnormal asymmetry brain organization. Another problem is that it is not clear how a lack of activation in the left posterior temporal regions associated with phonological processing is linked to the abnormal organization in the PT areas. Since sequential processing is specialized for the $\mathrm{LH}$, this theory can account for poor sequential processing in dyslexic children. However, the theory cannot account for poor bimanual motor coordination, since it excludes the involvement of the RH in dyslexia. The theory never discusses subtypes and visual problems, such as transporting letters in a word.

The magnocellular deficit theory tries to account for DD within the visual system. About $75 \%$ of dyslexics are estimated to have impairment in the magnocellular (M) system (Lovegrove et al., 1986). This system conveys information about motion and depth (Livingstone \& Hubel, 1988), and this may explain the experience of transporting letters and words that many dyslexic children report. It is not compatible with the left hemisphere 
dysfunction theory since some dyslexics that have impairment in the M-system typically exhibit normal leftward asymmetrical PT (Best \& Demb, 1999). In terms of minor cognitive impairment and poor bimanual coordination, the theory offers no explanation. Furthermore, this theory does not explain how a relatively minor deficit at a low level of the visual system affects complex reading at a high level in the cerebral cortex. More investigation is needed to clarify a relationship between the role of the M-system and reading.

All three theories previously discussed try to locate the causality of DD at a single locus or deficit in the brain. However, reading is a complex activity integrating multiple aspects of information processing in the brain, such as visual, phonological, semantic, and lexical processing, as well as comprehension, memory, etc. To integrate multiple processing, the two hemispheres must collaborate. The interhemispheric disconnection theory focuses on interhemispheric interaction and claims that callosal deficits cause DD (Gladstone et al., 1989; Rousselle \& Wolff, 1991; Summerfield \& Michie, 1993). While the role of the CC is not yet fully understood, we now believe that various cognitive functions, such as memory or attention, involve interhemispheric communication (Zaidel, 1995; Zaidel \& Sperry, 1974, 1977). Previous studies have shown that dyslexic children perform poorly in tactile and bimanual motor coordination tasks known to require interhemispheric communication. Summerfield and Michie (1993) found that the tactile performance on the Crossed condition and standardized reading scores were positively correlated with each other. Recent MRI studies (Robinchon \& Habib, 1998; Rumsey et al., 1996) have further supported this theory. They revealed that dyslexic brains show a thin genu or a larger 
isthmus of the CC compared to normal brains. Thus, convergent evidence from different methodologies indicates that a lack of efficient interhemispheric interaction contributes to a specific reading disability.

Because this theory involves both cerebral hemispheres, it is possible to accommodate a variety of cognitive impairments shown by $\mathrm{DD}$ children associated with cerebral specialization, such as phonological processing for the $\mathrm{LH}$, and visuo-spatial processing for the RH. However, because both anatomical and functional mechanisms of the $\mathrm{CC}$ have not been fully understood, it is not clear what role the $\mathrm{CC}$ has in cognitive functions. For example, children with callosal agenesis demonstrate the same poor phonological processing that DD children do; however, they have adequate reading ability. Perhaps they use alternative pathways, which are only available for this population but not for commissurotomized patients. Because phonological processing is highly specialized for the LH, it is not immediately clear why these children show phonological deficits. There is much speculation associated with the role of the $\mathrm{CC}$ in higher cognitive functions. More research based on the interhemispheric disconnection theory is needed to provide conclusive evidence of the possible involvement of the $\mathrm{CC}$ in DD. Although the interhemispheric disconnection theory focuses on inefficient interhemispheric communication, this theory does not exclude other theories, which tend to focus on a specific brain function or region. Thus, this theory can potentially account for five out of six primary characteristics of DD. Although this theory is not as well-known as other theories, there is sufficient evidence to warrant continuing study. 
We are beginning to understand that interhemispheric interaction is involved in higher mental functions (Banich \& Karol, 1992; Fabbro et al., 2001; Gladstone et al., 1989; Sugishita et al., 1992; Summerfield \& Michie, 1993; Temple \& Ilsley, 1994; Wolff et al., 1990; Zaidel, 1995). The present thesis explores the possibility of the involvement of interhemispheric interaction in DD using a variety of tasks associated with different callosal regions. The three experimental tasks include a tactile finger localization task associated with the posterior midbody and two visual cognitive tasks associated with the genu and the isthmus, respectively. The next chapter will discuss the rationale of this thesis project. 


\section{Chapter 8: Rationale}

The aim of this chapter is to describe the rationale for the present thesis. There were two hypotheses for this thesis: (1) DD is associated with inefficient interhemispheric communication; and (2) DD is a specific reading disorder. The first hypothesis was to further investigate the interhemispheric disconnection theory. As discussed in Chapter 6, this theory holds the view that DD is attributable to inefficient interhemispheric communication through the $\mathrm{CC}$ rather than dysfunction confined to the LH. Previous research associated with this theory that investigated DD took account of a single factor, such as bimanual motor coordination (Gladstone et al, 1989; Rousselle \& Wolff, 1991) or cross-finger localization (Quinn \& Geffen, 1986;; Summerfield \& Michie, 1993), in order to investigate DD. However, since DD manifests itself in diverse deficits, it is essential to take multiple characteristics into account to understand DD. A major challenge for the present thesis was to focus on three cerebral functions associated with the callosal regions and to attempt an understanding of DD by examining a combination of these functions.

The second hypothesis was motivated by the controversy as to whether DD is a specific reading disorder, or whether it is at the extreme edge of poor reading ability in the normal distribution (Stanovich, 1991; Swan \& Goswami, 1997). Since the definition of DD has not been firmly established, it is important to investigate in what respects DD is similar and dissimilar to general poor reading ability. 
This chapter is divided into four sections: (1) investigation of the interhemispheric disconnection theory, (2) investigation of the controversy in the literature, (3) tasks and predictions, and (4) strengths of the present thesis.

\subsection{Investigation the Interhemispheric Disconnection Theory}

There are three assumptions underlying the interhemispheric disconnection theory: (1) reading requires interhemispheric communication; (2) the $\mathrm{CC}$ is essential in interhemispheric communication; and (3) inefficient interhemispheric communication is associated with DD. These assumptions have been elucidated in a number of studies.

\subsubsection{First Assumption}

Ellis (1994) argued that reading, unlike speech, is not lateralized in the brain, and that multiple brain systems involving both hemispheres are necessary for processing reading. These brain systems include visuo-spatial and semantic systems that developed during the evolution of the human species; and were not designed for reading. Miller (1991) also argued that reading was probably derived from spoken language and has slowly evolved in human cultures. The traditional view of hemispheric specialization (discussed in Chapter 3) assumes that systems for reading are mainly located in the LH (Galaburda et al., 1985; Pugh et al, 2000), while the alternative view, that both cerebral hemispheres are involved in reading, and thus, interhemispheric communication is essential for reading, is not well-known but has many advocates (Banich \& Karol, 1992; Gladstone \& Best, 1985; Summerfield \& Michie, 1993; Zaidel, 1995). 
To explore the latter view, Banich and Karol (1992) compared unilateral and bilateral information processing using a rhyming judgment task in a visual half-field presentation (see Appendix A). In their task, a target (e.g., key) was presented in the center of the screen; and in the unilateral presentation, two probes (stimuli, e.g., bee/mug) were presented together either in the $\mathrm{LVF} / \mathrm{RH}$ or in the $\mathrm{RVF} / \mathrm{LH}$. In the bilateral presentation, one probe was presented on each VF simultaneously (i.e., bee on the LVF and mug on the RVF). Probes were presented for $200 \mathrm{msec}$. The participant was asked to decide if either of two probes presented rhymed with the target (e.g., key) after viewing the target word for one second. The probes were identical (e.g., bee-bee/mug-mug) on some trials across the conditions. This redundancy of information facilitated performance in the RVF/LH (i.e., faster RT in the same probes than the different probes) but not in the LVF/RH in the unilateral presentation. In the bilateral presentation, however, the effect was relative (i.e., less than in the RVF/LH but more than in the LVF/RH). When the font and case of the probe were manipulated, this manipulation altered the pattern of the results only in the bilateral presentation. Specifically, when the probe was presented in a different font and case (e.g., BEE-bee/mug-MUG), the advantage in the redundancy of information disappeared in the bilateral presentation. The authors argued that conflict between the physical form and lexical status/meaning between the hemispheres in the bilateral presentation supports the view that interaction between the two hemispheres occurs during reading processing because each VF processed different information in this condition. These authors suggested that looking at the function of either hemisphere alone is not sufficient to understand the nature of reading processing. 
Why is interhemispheric communication necessary for information processing? Banich and colleagues (Banich \& Belger, 1990; Belger \& Banich, 1992) subsequently investigated this question, hypothesizing that interhemispheric communication is more efficient for computationally complex tasks than less complex tasks. Using a visual halffield paradigm, they compared a physical-identity (e.g., A-A or A-B) and a name-identity task (e.g., A-a or A-b). In the physical identity task, different letters (A/B) were presented off the central fixation on both the VFs as probes, and a target letter (A) was located below either probe. Both probe and target were always presented in upper case. The participants were asked to press the button if the probe and target matched. When the matched probe and target were presented in the same VF, only intrahemispheric communication was necessary. When the matched probe and target were presented in different VFs, interhemispheric communication was necessary. In the name-identity task (e.g., A-a, A-b), the probe was always presented in upper case and the target was always presented in lower case. The name-identity task required extra processing (i.e., phonological processing) in addition to physical comparisons compared to the physical-identity task (e.g., A-A, A-B vs. A-a, A-b). Thus, the name-identity task was more complex than the physical identity task. Their findings demonstrated that intrahemispheric communication is superior when performing a physical-identity task, whereas interhemispheric communication is superior when performing a name-identity task. Thus, the authors concluded that interhemispheric communication is advantageous for computationally complex tasks, and intrahemispheric communication is advantageous for less complex tasks. The same advantage of 
interhemispheric communication for the name-identity task was also found in children (age range of 6-14 years) using the same paradigm (Banich, Passarotti, \& Janes, 2000).

Further evidence to support the assumption that reading requires interhemispheric communication has been provided from two neuroimaging studies. As discussed in Chapter 5.3.5, an fMRI study (Shaywitz et al., 1995) was conducted to examine a hypothesis that language function is likely to be lateralized in males and to be represented bilaterally in females. In the study, 38 normal right-handed subjects (19 males and 19 females, mean age of 28.5 years for the males and 24.0 years for the females) performed four distinct samedifferent judgment tasks on visually presented stimuli during fMRI scanning. The four tasks were to identify: (1) whether two lines were in the same orientation; (2) whether two sets of consonant strings were the same case; (3) whether two nonsense words rhymed; and (4) whether two words came from the same semantic category. Females but not males showed bilateral activation around Broca's area during the rhyming judgment task but not the other tasks. This bilateral activation implies that interhemispheric communication is necessary to process the visually presented linguistic stimuli in at least some females.

As discussed in Chapter 5, Clarke and Zaidel (1994) used an MRI technique to demonstrate a significant negative correlation between the size of the isthmus (connecting Wernicke's area to the homologous RH area, corresponding to 6 in Figure 5.2) and priming effects from a primed lexical decision task (LDT) with 60 graduate students (equal numbers of left- and right-handed males and females). An LDT is a task in which a participant is required to make judgments as to whether a presented item is a real word. The spreading activation theory (e.g., Anderson, 1976; 1983; Collins \& Quillian, 1969; Collins \& Loftus, 
1975; Neely, 1977) assumes that priming effects (facilitation) occur when a pair of words (e.g., cat-dog) is associatively/semantically linked in the mental lexicon, but do not occur when a pair of words (e.g., cat-bus) has little or no linkage. According to this theory, when a prime word (cat) is input into the mental lexicon, the activation spreads out to the related words of the prime including the target (dog). Thus, in a primed LDT, when the target word (dog) is input into the mental lexicon, it has already been activated (i.e., warmed up). This activation results in faster response to the target in an associated pair of words (i.e., priming effects). Clarke and Zaidel reported that the right-handed males who obtained greater priming effects from the target presented in the RVF/LH tended to have a smaller isthmus area than left-handed males; no correlation between isthmus size and level of priming was found in left- or right-handed females. A smaller isthmus suggests less capacity of interhemispheric communication. Thus, the observed correlation is compatible with the view that semantic processing, at least using linguistic stimuli, is lateralized to the LH in right-handed males. It also suggests that left-handed males and females tend to have bilateral representation in processing semantic information. Taken together, these two studies suggest that interhemispheric communication is essential in reading for females and left-handed males.

As discussed in Chapter 3.1, alexia (reading disability caused by brain damage) research in the English and Japanese languages (Benson, 1981; Benson \& Geschwind, 1969; Sugishita et al., 1992) found that pure alexia (disruption of reading without writing or speech disability) typically results from lesions involving both the left posterior occipital lobe and the splenium of the $\mathrm{CC}$ (corresponding to 7 in Figure 5.2). It was most of the time 
accompanied by right hemianopsia (blindness in the RVF). The involvement of the splenium in pure alexia suggests that visual input from the RH to the $\mathrm{LH}$ is necessary for reading. In the case of Japanese alexic patients, when a lesion is located in the posterior CC including the splenium, it tends to cause more severe reading impairment in Kana (phonogram) than Kanji (ideogram) reading (Sugishita, Iwata, Toyokura, Yoshioka, Yamada, 1978; Sugishita, Yoshioka, \& Kawamura, 1986). This finding indicates that visual input through the splenium has a role in phonological processing.

Taken together, these studies support the first assumption: that reading typically requires interhemispheric communication (Banich \& Belger, 1990; Belger \& Banich, 1992; Banich \& Karol, 1992, Benson, 1981; Benson \& Geschwind, 1969; Clarke \& Zaidel, 1994; Shaywitz et al., 1995, Sugishita, Iwata et al., 1978; Sugishita et al., 1986, Sugishita et al., 1992).

\subsubsection{Second Assumption}

As to the second assumption, that the $\mathrm{CC}$ is essential in interhemispheric communication, studies with commissurotomized patients have provided evidence for callosal roles in visual information transfer between the two hemispheres (Bogen \& Vogel, 1962; Bogen \& Gazzaniga, 1965; Sperry, 1962, 1968). For instance, Sperry (1968) examined a commissurotomized patient, NG, to see if NG could identify an object briefly flashed in either her LVF/RH or RVF/LH using a tachistoscope (see Appendix A). The findings demonstrated that she could name an object visually presented in her RVF/LH, but could not verbally identify the same object flashed in her LVF/RH; however, she could correctly point to the object flashed in her LVF/RH in an array of pictures using her left 
hand. Sperry explained these findings such that when an object flashed in her RVF/LH, the visual information was directly projected into the LH (contralateral hemisphere to the presented VF), which normally controls speech, and thus, she could name the object; however, when an object flashed in her LVF/RH, the information was first projected into the $\mathrm{RH}$, and needed to be transferred to the $\mathrm{LH}$ via the $\mathrm{CC}$ in order to be named. However, her sectioned $\mathrm{CC}$ blocked transfer of the information. In the case of pointing to an object, when an object was flashed in the LVF/RH, the information was projected into the $\mathrm{RH}$ and since the RH controls the motor movement of the left hand, interhemispheric information transfer was not involved in pointing to the object using the left hand. Furthermore, NG was asked to name a common object by touching without viewing. NG could not name the object when she held it in her left hand/RH, but as soon as she held the same object in her right hand, she could name it.

In a finger localization task, Geffen et al. (1985) also confirmed disconnection effects in tactile information processing with commissurotomized patients. The task involved imitating finger sequences on the same or opposite hand when fingers were sequentially touched without viewing (see Chapter 5.3.1). In the task, interhemispheric communication was required only when the finger localization sequence was imitated using the opposite hand (Crossed condition) but not when using the same hand (Uncrossed condition). The commissurotomized patients performed more poorly on the Crossed than Uncrossed condition.

Another example of a disconnection effect is left-hand apraxia (inability to respond to verbal commands); (Zaidel \& Sperry 1974; Aglioti et al., 1998) and poor performance of 
the right hand in copying geometric figures (Loring et al., 1989; Zaidel \& Sperry, 1977). The most common explanation for left-hand apraxia (Watson \& Heilman, 1983) is that since the LH understands verbal commands, the information needs to be transferred from the $\mathrm{LH}$ to the $\mathrm{RH}$ via the $\mathrm{CC}$ because the $\mathrm{RH}$ controls motor movement of the left hand; however, since information transfer is blocked due to callosal sectioning, left-hand apraxia appears. The reverse scenario is poor performance in copying geographic figures using the right hand. Because the $\mathrm{RH}$ is specialized for visuo-spatial processing, information about geographic figures needs to be transferred from the $\mathrm{RH}$ to the $\mathrm{LH}$ via the $\mathrm{CC}$ in order to use the right hand; however, this transfer is blocked due to callosal sectioning. These results suggest that the $\mathrm{CC}$ is necessary for both visual and tactile information transfer interhemispherically.

Furthermore, commissurotomized patients demonstrated disconnection effects in bimanual motor coordination (Preilowski, 1972, 1975). In a crank-handle task (see chapter 5.3.2), commissurotomized patients were asked to trace lines at different angles drawn on paper. In one task, they were asked to trace a line at $112.5^{\circ}$, which required turning a knob with one hand twice as fast as with the other hand. Thus, the task required bimanual motor coordination to trace a line at that angle. The commissurotomized patients demonstrated slower and less accurate performance than the normal controls and epileptic patients, particularly without viewing, in which there was no visual cue available for tracing the lines. This suggests that the commissurotomized patients relied on visual cues in order to coordinate their hands, and without the visual cues, their hands appeared to be unable to communicate with each other to complete a task. Jeeves and Silver (1988) used this crank- 
handle task to examine bimanual motor coordination in two individuals with callosal agenesis (congenital absence of the $\mathrm{CC}$ ) who had normal cognitive functions. They demonstrated very slow and inaccurate performance in tracing the lines. In particular, their poor performance manifested itself in tracing a line at $112.5^{\circ}$ angle without viewing, as did that of the commissurotomized patients. This suggests that the $\mathrm{CC}$ plays a role in bimanual motor coordination.

Taken together, research with clinical populations generally supports the view that the $\mathrm{CC}$ is essential for interhemispheric communication in information processing (Aglioti et al., 1998; Bogen \& Vogel, 1962; Bogen \& Gazzaniga, 1965; Jeeves \& Silver, 1988; Loring et al., 1989; Preilowski, 1972, 1975; Sperry, 1962, 1986; Zaidel \& Sperry, 1974, 1977).

\subsubsection{Third Assumption}

As to the third assumption that the inefficient interhemispheric communication is associated with DD, several researchers in the dyslexic literature support this assumption (Fabbro et al, 2001; Gladstone et al., 1989; Summerfield \& Michie, 1993; Wolff et al., 1984, 1990, Geffen et al., 1985). Gladstone et al. (1989) tested an ability of bimanual motor coordination in DD children (mean age of 12.08 years). The task was similar to the crankhandle task described earlier. In this task, however, the DD children turned both knobs in a clockwise direction (unidirectional condition) to follow paths at $26.5^{\circ}$ and $63.5^{\circ}$ with the speed ratio of the left and right hand of 1:2 and 2:1, respectively. To follow the paths at $116.5^{\circ}$ and $153.5^{\circ}$, they needed to turn each knob in different directions (bi-directional condition) with a speed ratio of the left and right hand of $1: 2$ and $2: 1$, respectively. That is, 
to follow a path at $116.5^{\circ}$, the left hand needed to move twice as fast as the right hand, and at the same time, one hand had to turn the knob in a clockwise direction, while the other hand had to turn it in a counter clockwise direction (bi-directional condition). Thus, the bidirectional task required bimanual motor coordination. With viewing, although the DD children performed similarly to the controls in the unidirectional condition, they demonstrated slower and less accurate performance than the controls (mean age of 12.3 years) in the bidirectional condition. Without viewing, however, they were unable to maintain the bi-directional movements (i.e., mirror movements), indicating that the DD children could correct the direction with visual monitoring, but could not do so without visual cues. These results were compatible with those demonstrated by commissurotomized patients and individuals with callosal agenesis in that these three populations failed to coordinate their bimanual motor movement in the absence of visual feedback. This suggests that DD children might have some impairment in callosal connectivity.

Poor phonological processing is considered to be the main characteristic of DD (e.g., Racket al., 1993; Snowling, 1995, 2000). Interestingly, children with callosal agenesis share this problem with DD children (Dennis, 1981; Jeeves \& Temple, 1987; Temple \& Ilsley, 1994), although they have relatively good reading ability. This common poor phonological processing indicates that $\mathrm{DD}$ children might have some impairment in callosal connectivity. Furthermore, Summerfield and Michie (1993) reported a significant positive correlation between reading test scores and finger localization task performance on the Crossed condition but not the Uncrossed condition. This result suggests that there is a link between interhemispheric communication and reading. In addition, MRI studies (Duara et 
al., 1991; Hynd et al., 1995; Robichon et al., 2000; Robichon \& Habib, 1998; Rumsey et al., 1996) have shown that adult DD brains have morphological abnormalities involving the CC. Although these MRI studies have not yet been replicated in DD children, they suggest that DD children might also have abnormal callosal connectivity.

Taken together, previous studies support the third assumption that deficits in interhemispheric communication are associated with DD (Duara et al., 1991; Gladsone \& Best, 1986; Hynd et al., 1995; Robichon et al., 2000; Robichon \& Habib, 1998; Rumsey et al., 1996; Summerfield \& Michie, 1993; Wolff et al., 1984, 1990; Geffen et al., 1985).

In summary, the three assumptions underlying the interhemispheric disconnection theory of DD are supported by research findings: (1) reading requires interhemispheric communication; (2) the $\mathrm{CC}$ is essential in interhemispheric communication; and (3) deficits in interhemispheric communication are associated with DD. Thus, one may expect that DD children will perform more poorly on tasks which require interhemispheric communication than on those that do not.

\subsection{Investigation of the Controversy}

Regarding the second goal, as discussed in Chapter 1.3, there is controversy as to whether DD is a specific reading disorder that is qualitatively different from poor reading ability (Swan \& Goswami, 1997); or whether DD children are just extremely poor readers, and simply lie at the edge of the large population of poor readers (Stanovich, 1991; Stanovich et al., 1994). Given the lack of consensus on the definition of DD in the dyslexia literature, it is important to investigate this issue and to identify which aspects of DD are similar and dissimilar to poor reading ability. In the present thesis, it was decided to 
compare DD children to three control groups; (1) chronologically age-matched (CA) readers, (2) chronologically age-matched general poor (CAP) readers, and (3) reading agematched (RA) readers. The CAP readers were defined as those whose reading difficulty can be accounted for by a variety of factors, such as low intelligence, a lack of motivation or educational opportunity.

\subsection{Tasks and Predictions}

Three experiments were designed to investigate the two hypotheses outlined in sections 8.1 and 8.2. As discussed in Chapter 5.1, the callosal topography is specific. It is organized in an orderly manner from the anterior to the posterior regions corresponding to the cortical functions. This indicates that different callosal regions process different information associated with the cortical functions between the two hemispheres (De Lacoste et al., 1985; Pandya \& Seltzer, 1986). There is no standardized test to measure callosal functions. In the present thesis, each experiment focuses on a specific callosal region associated with a specific cortical function based on the unique topography of the $\mathrm{CC}$.

There were three callosal areas of interest for this thesis: the genu, the posterior midbody and the isthmus (each area is corresponding to the CC \# 2, \# 5 and \# 6 in Figure 5.2, respectively). The genu connects Broca's area to the homologous area in the $\mathrm{RH}$ (De Lacoste et al., 1985; Pandya \& Seltzer, 1986). Previous studies have suggested that Broca's area is associated with phonological processing in reading (Brunswick et al., 1999; Burton et al., 2000; Shaywitz et al., 1995). Thus, studying inefficient interhemispheric communication of phonological information may allow for the investigation of callosal 
connectivity associated with the genu. The posterior midbody connects the somato-sensory cortical area to the homologous area in the RH (De Lacoste et al., 1985; Pandya \& Seltzer, 1986). Thus, tactile tasks would allow for the investigation of deficits in interhemispheric communication associated with the posterior midbody. Finally, the isthmus connects Wernicke's area to the homologous area in the RH (De Lacoste et al., 1985; Pandya \& Seltzer, 1986). Alexia research (Friedman et al., 1993; Funnel, 1983; 1999; Mesulam, 1990; Patterson et al., 1985) has suggested that Wernicke's area is closely related to reading systems. Previous studies (Clarke \& Zaidel, 1994; Hecaen \& Albert, 1978; Hier \& Mohr, 1977 ) indicate that this area is also closely linked to semantic processing. Thus, semantic tasks would allow for the investigation of inefficient interhemispheric communication associated with the isthmus.

Three tasks were chosen based on cerebral functions associated with callosal regions: (1) a finger localization task (tactile, Experiment 1); (2) a rhyming judgment task (phonological, Experiment 2); and (3) a primed lexical decision task (semantic, Experiment 3). The tactile task was first conducted in Experiment 1 followed by the rhyming judgment task in Experiment 2 and the primed lexical judgment task in Experiment 3. The order of the three experiments was important. This was because previous studies (Fabbro et al., 2001; Gross-Glenn \& Rothenberg, 1984; Lindgren, 1978; Summerfield \& Michie, 1993) using a finger localization task provided evidence that DD is associated with inefficient interhemispheric information transfer. There was little direct empirical evidence using the phonological and semantic tasks associated with inefficient interhemispheric 
communication in DD. The demonstration of inefficient interhemispheric communication associated with DD using the tactile task established the foundation for the present thesis.

The sample included 19 DD students (mean age of 13.07 years), 25 CA students (mean age of 13.04 years), 10 CAP students (mean age of 13.08 years), and 17 YA (young age) students (mean age of 9.06 years). It should be noted that an RA (reading agematched) group could not be formed for this project, because the young students (ranging in age from 8 to 10 years) who participated in the project turned out to be above average readers for their age (mean reading age of 12.06 years). Thus, they formed a young age (YA) group instead of an RA group. A complete description of each group (e.g., sample size, gender, and handedness) as well as screening procedures will be provided in the next chapter.

\subsubsection{Finger Localization Task (Experiment 1)}

Experiment 1 was based on Summerfield and Michie's 1993 study using a finger localization task. The finger localization task has been used with a variety of populations including other clinical populations (Geffen et al., 1985; Geffen, Nilsson, Simpson, \& Jeeves, 1994) to examine tactile information transfer between hemispheres, normal age changes in callosal development (Galin, Diamond, \& Herron, 1977; Galin, Johnstone, Nakell, \& Herron, 1979; Quinn \& Geffen, 1986), and correlations between callosal developmental changes and different cognitive abilities including reading (Fletcher \& Satz, 1980; Fletcher, Taylor, Morris, \& Satz, 1982; Lindgren, 1978; Moore et al., 1996; Zung, 1986). 
As discussed earlier, somato-sensory information is believed to be transferred between hemispheres through the posterior midbody of the $\mathrm{CC}$ (corresponding to the $\mathrm{CC} \#$ 5 in Figure 5.2) (De Lacoste et al., 1985; Pandya \& Seltzer, 1986). Since there is no ipsilateral input (e.g., left hand to the LH) from the distal portions of the limbs (hand and feet) in the somatosensory cortex, tactile information requires the projecting of input to the contralateral hemisphere (e.g., left hand to the $\mathrm{RH}$ ). The finger localization task (see Chapter 5.3.1) involves imitating finger sequences on the same or opposite hand when fingers are sequentially touched without viewing. According to the classical interpretation (Bogen \& Vogel, 1962; Bogen \& Gazzaniga, 1965; Sperry, 1962, 1968), when a response is required by the opposite hand, the tactile information needs to be transferred from one hemisphere to the other via the $\mathrm{CC}$ in order to move the opposite hand (Crossed condition). However, when a response is required by the same hand, callosal transfer is not necessary to move the same hand (Uncrossed condition). Most studies of normal adults reported a loss of 7\% tactile information in accuracy in the Crossed condition. In clinical populations, Geffen et al. (1985) reported that patients with complete and partial commissurotomy showed a loss of $82 \%$ and $28 \%$ tactile information, respectively. Geffen et al. (1994) also reported a loss of tactile information in children with callosal agenesis. The degree of loss of information in this study was dependent on the age of testing. Based on MR data, children at eight years of age had an accuracy rate of $40 \%$ in the Crossed condition vs. $100 \%$ accuracy in the Uncrossed condition. Accuracy increased among 15 year-olds to $58 \%$ in the Crossed condition. This suggests that even when the CC is absent in whole or in part, interhemispheric communication increases with age. To summarize, callosal 
transfer is normally associated with a small but significant degradation of information in tactile cross-localization tasks; when the $\mathrm{CC}$ is damaged or missing from birth, the degradation of information is much greater, but may be reduced with age.

Developmental studies (Galin et al., 1977; Galin et al., 1979; Quinn \& Geffen, 1986) found that the efficiency of interhemispheric transfer of tactile information normally improves with age. Quinn and Geffen (1986) tested developmental improvement in the efficiency of tactile callosal information transfer with children between 5 and 11 years of age in a finger localization task. They found that callosal efficiency for tactile information transfer increases with age (i.e., from $53 \%$ to $88 \%$ accuracy with a one-finger task on the Crossed condition). By the age of 11 years, children showed a loss of $12 \%$ of tactile information in the Crossed condition, which was close to the percentage observed in normal adults (7\%). The authors suggested that this similar performance level to adults is consistent with the completion of major myelination of the $\mathrm{CC}$ around the age of 12 and that callosal function develops slowly with the completion of callosal myelination around 20 years.

Although finger localization would appear to have no obvious link with reading, previous studies (Fletcher et al., 1982; O'Donnell, Lindgren, 1978; Moore et al., 1996; 1983; Zung, 1986) have found that finger localization on the Crossed condition does positively correlate with reading ability. Summerfield and Michie (1993) studied 16 DD boys (mean age of 9.4 years). They found that the DD boys performed less accurately than a younger reading age-matched control group (mean age of 7.3 years) on the Crossed condition. Fabbro et al. (2001) reported that DD children $(n=8$, mean age of 11.8 years $)$ 
performed similarly to a younger reading age-matched control group (mean age of 7.4 years) on the Crossed condition, despite the fact that children at this age should perform similarly to adults. These results suggest that information transfer through this callosal region is strongly associated with reading ability in some ways. They also suggest that inefficient interhemispheric communication or delayed maturation in this region may result in reading disability.

With respect to experimental variables, correct responses and sequence errors of finger localization were used as dependent variables. Since one of the characteristics of DD children is poor sequence processing (Bakker, 1973), the number of finger sequences (i.e., 2-Finger, 3-Finger, and 4-Finger sequence) was manipulated as an independent variable. The sequence errors were measured by error responses, in which all fingers were correctly identified but in the wrong order. Thus, the Group (DD, CA, CAP, YA), Condition (Uncrossed, Crossed), and Finger Sequence (two, three, four) were manipulated as the independent variables.

With respect to the first hypothesis of this thesis, tactile information transfer through the $\mathrm{CC}$ is associated with a small but reliable information degradation (Geffen et al., 1985). It was, therefore, predicted that all four groups would show lower scores on the Crossed than the Uncrossed condition. In addition, the interhemispheric disconnection theory assumes that inefficient interhemispheric communication is associated with DD. It was, therefore, predicted that the DD group would show more information degradation than the normal control groups in the Crossed condition. Furthermore, developmental studies (Galin et al., 1977, Galin et al., 1979; Quinn \& Geffen, 1986) have provided evidence that 
callosal efficiency is positively correlated with age in tactile information transfer. Thus, it was predicted that the young age group would show more information degradation than the older groups. Finally, if DD is qualitatively different from general poor reading ability, it was expected that the DD group would show dissimilar performance patterns to the poor readers group.

\subsubsection{Rhyming Judgment Task (Experiment 2)}

A rhyming judgment task using a visual half-field paradigm was chosen to investigate phonological information processing. Poor phonological processing is widely believed to be the primary deficit of DD (e.g., Ellis, 1991, 1994; Olson et al., 1985; Racket al., 1992; Snowling, 1995, 2000). Functional MRI studies with dyslexic adults (Milne et al., 2002; Pugh et al., 2000; Shaywitz et al., 1998) have found abnormal activation around Broca's area during phonological processing in a rhyming task. In particular, an fMRI study (Shaywitz et al., 2002) with DD children found bilateral abnormal activation in the anterior regions including Broca's area. Hynd et al. (1995) found a smaller genu in dyslexic adult brains than age-matched normal brains on average in an MRI study. The neuroimaging studies suggest that DD children might have functional and structural abnormalities in the $\mathrm{CC}$ affecting anterior brain regions including Broca's area.

A series of Banich studies (Banich \& Belger, 1990; Belger \& Banich, 1992; Banich, Passarotti, \& Jane, 2000) demonstrated that interhemispheric communication is efficient on computationally complex tasks. Because reading is a complex task, their findings suggest that interhemispheric communication is efficient for phonological processing. Furthermore, clinical evidence suggests that phonological processing requires bilateral activation. For 
example, a sample of children with callosal agenesis (Temple \& Ilsley, 1994; Temple et al., 1990) demonstrated poor phonological processing, and Japanese alexic patients (Sugishita et al., 1978, 1986) with lesions of the posterior CC including the splenium demonstrated greater reading impairment for Kana (phonogram) than Kanji (ideogram) reading. These studies suggest that information transfer through the $\mathrm{CC}$ is associated with phonological processing in Japanese.

The advantage of visual half-field presentation paradigm is that it allows the investigation of the laterality function of each cerebral hemisphere in the visual modality, and interactions of the two cerebral hemispheres at the same time. This paradigm has been used to investigate laterality and interhemispheric communication with a normal population (e.g., Hellige, 1983, 993; Karol \& Banich, 1992; Levine \& Banich, 1982) as well as clinical populations including a commissurotomized group (e.g., Tassinari et al., 1994) and a callosal agenesis group (Corballis \& Finlay, 2000). The disadvantage of this method is that information must be presented for $200 \mathrm{msec}$ or less because this is the amount of time required to move the eyes from one position to another; only if the eyes maintain fixation on a single point can it be ensured that an image in each visual field (VF) projects to the contralateral hemisphere (Banich, 1997).

In the present study, stimuli were presented on a screen between $1.5^{\circ}$ and $2.5^{\circ}$ horizontally to the left or right of the center fixation dot on the computer screen. Thus, even if small eye movements occurred, projection of the image to the opposite hemisphere was ensured. In the Unilateral condition, a pair of words was sequentially presented in either the $\mathrm{RVF} / \mathrm{LH}$ or the LVF/RH. In the Bilateral condition, one of the pair of words was presented 
in the RVF/LH; and sequentially, the other word was presented in the LVF/RH, and vice versa. The task was to click the 'Yes' label on the mouse when the words rhymed, and the 'No' label when they did not. Thus, interhemispheric communication was necessary to compare the two words presented in the Bilateral condition but not in the Unilateral Condition.

With respect to experimental variables, accuracy and reaction time (RT) to the second word of the pair were recorded as the dependent variables. The Group (DD, CA, CAP, YA), Condition (Unilateral and Bilateral) and Visual Field (Left and Right) were manipulated as the independent variables.

A right visual superiority effect is a typical finding in the visual half-field paradigm when linguistic stimuli are used (e.g., Gross et al., 1978; Levine \& Banich, 1982; Krutsch \& McKeever, 1990; Ortells et al., 1998; Yeni-Komshian et al., 1975). That is, when a subject fixates on a central point, a verbal stimulus (e.g., word) presented in the right visual-field (RVF)/LH is responded to faster and more accurately than one presented in the left visual field (LVF)/RH. This phenomenon is accounted for by the fact that in the visual system, an image presented in one VF is projected to the contralateral cerebral hemisphere. Thus, when a word is presented in the RVF/LH, the information is directly projected in the $\mathrm{LH}$, whereas when a word is presented in the LVF/RH, the information needs to be transferred to the opposite hemisphere to be processed via the CC. Callosal transfer takes time and causes some degradation of information (Bradshaw \& Nettleton, 1981). Thus, the RVF superiority effect is explained as a reflection of the advantage of direct access to the LH in linguistic stimuli. 
With respect to the two hypotheses of this thesis, a RVF superiority effect was predicted overall. Since phonological skills are a good predictor for reading ability (e.g., Adams, 1990; Baron, 1979; Doctor \& Coltheart, 1980; Ehri \& Wilce, 1979; Stanovich, 1982), it was predicted that, overall, the good reader groups would show faster and the higher scores in accuracy than the poor reader groups. A series of Banich studies (Banich \& Belger, 1990; Belger \& Banich, 1992; Banich et al, 2000) demonstrated that interhemispheric communication is advantageous for complicated tasks. It was, therefore, predicted that the control groups would respond faster and show higher scores in accuracy in the Bilateral than the Unilateral Condition. The interhemispheric disconnection theory assumes that inefficient interhemispheric communication is associated with DD. It was, therefore, predicted that the DD group would not show the advantage of bilateral presentation. Furthermore, developmental studies (Galin et al., 1977, 1979; Quinn \& Geffen, 1986) suggest that callosal efficiency improves with age. It was, therefore, predicted that the young age group also would not show the advantage of bilateral presentation. Finally, if DD differs qualitatively from general poor reading ability, it was expected that the DD group would show different patterns of performance from the CAP group.

\subsubsection{Primed Lexical Decision Task (Experiment 3)}

A primed lexical decision task (LDT) using a visual half-field paradigm was chosen to investigate semantic information processing associated with DD. Many researchers believe that the primary reading problem in $\mathrm{DD}$ is associated with phonological processing (e.g., Rack et al., 1992; Snowling, 1995, 2000) rather than semantic processing. However, 
since the left posterior temporal-parietal regions including Wernicke's area are closely related to reading systems in general, including semantic processing (Coltheart, 1980, 1983; Friedman et al., 1993; Saffran, 1985), the demonstration of abnormal activation in DD brains in this area suggests that DD children might have deficits in semantic processing.

In contrast to phonological processing, hemispheric specialization for semantic processing is not clear-cut. That is, the $\mathrm{RH}$, like the $\mathrm{LH}$, seems to be able to process semantic information (Chiarello \& Beeman, 1998). Ample evidence for this conclusion has been provided from studies with commissurotomized patients (e.g., Baynes, Tramo, \& Gazzaniga, 1991; Baynes, Wessinger, \& Gazzaniga, 1994; Eviatar \& Zaidel, 1991;Eviatar, Menn, \& Zaidel, 1990). For example, Zaidel and Peters (1981) found that the isolated RH of commissurotomized patients, LB and NG, were able to understand printed words by matching them with pictures of the objects. Gazzaniga, Ledoux, and Wilson (1977) demonstrated that the RH of a commissurotomized patient, PS, could comprehend verbs and respond to printed commands. Additional evidence for semantic processing by the $\mathrm{RH}$ has been also provided from aphasia research, i.e., disruption of this area results in a comprehension defect for spoken language (Wernicke's aphasia) (e.g., Grossi, Trojano, Chiacchio et al., 1991; Hecaen \& Albert, 1978; Hier \& Mohr, 1977; Benson, 1993). Studies with normal populations using a primed LDT paradigm (Burgess \& Simpson, 1988; Chiarello, 1985; Chiarello, Burgess, Richards, \& Pollock, 1990; Chiarello \& Richards, 1992; Clarke \& Zaidel, 1994; Koivisto \& Laine, 2000) have also demonstrated that both the right and left hemispheres have access to semantic information in the mental lexicon. These studies suggest that the $\mathrm{RH}$ is involved in reading processing semantically. 
The isthmus (corresponding to the $\mathrm{CC}$ region \# 6 in Figure 5.2) connects the left posterior temporal-parietal area of the cortex, including Wernicke's area, to the homologous area in the RH (De Lacoste et al., 1985; Pandya \& Seltzer, 1986). As discussed in Chapter 3.1, alexia research (Coltheart, 1980, 1983; Saffran, 1985) has provided evidence that a lesion in Wernicke's area also causes a deep alexia (evidenced by semantic errors in reading). These findings suggest that Wernicke's area is involved in semantic processing for both spoken language and reading. Clarke and Zaidel (1994) found a significant negative correlation between the size of the isthmus and the magnitude of priming effects in normal right-handed males but not in left-handed males and females in a primed LDT. They concluded that semantic processing is lateralized to the $\mathrm{LH}$ in right-handed males, whereas left-handed males and females are likely to have bilateral processing of semantic information. This result suggests that more than half of the population activate both hemispheres during semantic processing.

Neuroimaging studies (Brunswick, et al, 1999; Corina et al., 2001; Pugh et al., 2000; Shaywitz et al., 1998, 2002) have provided evidence that dyslexic adults and children fail to activate Wernicke's area during phonological tasks, while normal readers do activate this area. This finding suggests that DD adults and children have an impairment involving this brain region. Furthermore, MRI studies (Robichon \& Habib, 1998; Rumsey et al., 1996) found that the isthmus in dyslexic adult brains was larger or more rounded than in the controls. This morphological abnormality in the dyslexic brains suggests that there may be inefficient interhemispheric communication associated with Wernicke's area in DD adults and children. 
As discussed in Chapter 5, an LDT is a task in which a participant is required to make a judgment as to whether a presented item is a real word. Priming effects (facilitation effects) are believed to reflect the strength of word relatedness in the mental lexicon (Anderson, 1983; Collins \& Quillian, 1969; Collins \& Loftus, 1975; Neely, 1991). The magnitude of the priming effect can be measured by the difference between the associated (e.g., cat-dog) and non-associated (e.g., pen-bus) pair of words in terms of response time (i.e., RT) and accuracy to the target word (i.e., second word). Thus, the magnitude of the priming effect depends on how closely a pair of words is associated/related. In a primed LDT, a pair of words (prime and target) are presented in either VF (Unilateral Condition); or when a prime word is presented in one VF, the target is presented to the opposite VF (Bilateral condition). For example, in the Unilateral Condition, both cat (prime) and dog (target) are presented in either the $\mathrm{RVF} / \mathrm{LH}$ or the $\mathrm{LVF} / \mathrm{RH}$. In the Bilateral condition, when cat (prime) is presented in the $\mathrm{RVF} / \mathrm{LH}$, dog (target) is presented in the LVF/RH, or vice versa. The prime and target are either a pair of words (e.g., cat-dog, pen-bus) or a pair comprising a word and nonword (e.g., hat-tas). In the visual system, an image presented in each VF is projected to the contralateral cerebral hemisphere. Thus, when prime and target are presented in the one VF (Unilateral Condition), interhemispheric communication does not require to make lexical judgments and obtain priming effects. In contrast, when prime and target are presented separately in each VF (Bilateral condition), interhemispheric communication is necessary to make lexical judgments and obtain priming effects.

In the present study, the experimental variables were accuracy and RT to the target. The Group (DD, CA, CAP, YA), Condition (Unilateral and Bilateral), Visual Field (Left 
and Right), and Stimulus Pair (Associated, Non-Associated, Word and Nonword) were manipulated as the independent variables.

With respect to the two hypotheses of this thesis, since both the LH and the RH seem to have access to semantic information (Zaidel, 1978, 1982; Burgess \& Simpson, 1988; Chiarello, 1985, Chiarello et al., 1990; Chiarello \& Richards, 1992; Clarke \& Zaidel, 1994; Koivisto \& Laine, 2000), it was predicted that priming effects would be observed in the RVF/LH and LVF/RH presentation in the Unilateral Condition across the groups. Since semantic processing is closely related to reading (Adams, 1990), it was predicted that overall, the good reader groups would demonstrate faster response time and higher accuracy than the poor reader groups. If interhemispheric communication enhances efficiency of semantic processing (Banich \& Belger, 1990; Belger \& Banich, 1992), it was predicted that the normal control groups would demonstrate faster response time and higher accuracy in the Bilateral condition than the Unilateral Condition. If inefficient interhemispheric communication is associated with DD, it was predicted that the DD group would not demonstrate efficient information processing. In addition, if callosal efficiency is associated with callosal development (Quinn \& Geffen, 1986), it was predicted that the young age group would not show efficient information processing. Finally, if DD qualitatively differs from poor reading ability, it was expected that the DD group would show different patterns of performance from the CAP group.

\subsection{Strength of the Present Thesis}

The strength of this thesis is that all the experimental tasks investigated relationships between DD and deficits in information processing between the two 
hemispheres associated with the unique topography of the $\mathrm{CC}$, which is divided into regions that may be functionally distinct and related to cerebral functions. Each experiment focused on a different $\mathrm{CC}$ region that has been implicated in DD. The phonological and semantic processing tasks have more obvious connections with reading; in particular, the phonological task addresses a recognized weakness in the DD population. Although the finger localization task would appear to have no obvious link with reading, previous studies using this task (Fletcher et al., 1982; Lindgren, 1978; Moore et al., 1996; Summerfield \& Michie, 1993; Zung, 1986) revealed that there is a link between reading ability and somatomotor skills. Replication of this tactile deficit in the DD group in the Crossed but not the Uncrossed condition provides an important foundation for this thesis.

Another strength of this thesis is that because three cerebral functions were taken into account to investigate DD, a single or combination of findings from the three Experiments were able to provide more comprehensive understanding of DD than research focusing on a single deficit of DD. The variety of experimental tasks also allowed the comparisons between DD and poor reading ability by examining different aspects of reading ability.

In summary, a review of the literature supports the three assumptions underlying the interhemispheric disconnection theory. The three experiments explored this theory. They sought to differentiate DD from poor reading ability by examining different aspects of reading processing. 


\section{Chapter 9: Sample}

The aim of this chapter is to describe the sample used for Experiments 10,11 and 12 including the sampling criteria and procedure. As discussed in Chapter 1.1, although the World Federation of Neurology (Critchley, 1970) set the definition for DD, there is in fact no clear and accepted definition of DD. This is mainly because the etiology of DD is unknown, and DD children comprise heterogeneous groups. In the last 30 years, clinical psychologists, educators and researchers have usually used a discrepancy between IQ and expected reading ability as an operational definition to identify DD children. Although the most common cut-off point is an IQ of 90 or above and a reading age at least two years behind the child's chronological age group (Backes et al., 2002; Corina et al., 2001; Drake, 1978; Gladstone et al., 1989; Hynd et al., 1992; Shaywitz et al., 2002; Swan \& Goswami, 1997), it is arbitrary (Snowling, 2000); and has resulted in the wide range of identified prevalence of DD, i.e., 3-10\% (Yule et al., 1974). Using this traditional reading-IQ discrepancy, it is difficult to dissociate DD children with relatively low IQ (close to IQ of 90) from poor readers (no dyslexia), who have reading difficulty and below average IQ (close to an IQ of 90). Thus, it is possible that some previous studies included poor readers (no dyslexia) in a DD group, but other studies did not, which might result in different findings: some researchers found that DD children showed worse performance in reading nonwords than a reading age-matched group (Frith \& Snowling, 1983; Olson et al., 1985; Rack et al., 1992, Snowling, 1995), while others did not (Beech \& Harding, 1984; Vellutino \& Scanlon, 1987). The IQ-discrepancy criteria has also created a controversy as to whether DD is a specific reading disorder (Frith \& Snowling, 
1983; Swan \& Goswami, 1997; Snowling, 1995, 2000) or lies at the extreme edge of poor readers (Fletcher et al., 1994; Stanovich, 1991; Stanovich \& Siegel, 1994). One of the goals of the present thesis was to dissociate DD from general poor reading ability by setting stringent criteria for the project. The criteria are discussed in the final grouping (see section 9.1.3).

The chapter is divided into four sections: (1) Sampling, (2) Handedness, (3) Verbal and Non-Verbal Assessment Materials, (4) Confirming Groupings, and (5) Investigation of Sub-types of Developmental Dyslexia.

\subsection{Sampling}

All participants were recruited from the following sources: (1) the OttawaCarleton District School Board (OCDSB) and the Ottawa-Carleton Catholic School Board (OCCSB), mainly for chronological age-matched (CA) readers, chronological agematched poor (CAP) readers, reading age-matched $(\mathrm{RA})$ readers, and some developmental dyslexic (DD) readers; (2) the Learning Disabilities Association of Ottawa for DD readers; (3) the Ontario Provincial Demonstration Schools mainly for DD readers and some CAP readers; and (4) acquaintances of the author for DD readers.

The OCDSB is the largest school board in Eastern Ontario serving approximately 80,000 students across 118 elementary and 27 secondary school programs including Special Education program support (Ottawa-Carleton District School Board, 2004). The OCCSB has 80 schools serving approximately 40,000 students across the region of Ottawa-Carleton, and offers elementary and secondary school programs including Special Education (Ottawa-Carleton Catholic School Board, 2004). The Ontario 
Provincial Demonstration Schools funded by the Ontario Provincial Government provide educational programs for approximately 120 students. This is a specialized residential school program for students with learning disabilities (LD), who demonstrate severe difficulties in reading, writing, spelling and mathematics skills in general classrooms. Students (between grades 4 and 11) are admitted to these schools on the recommendation of their local school board as having a specific learning disability which can be seen in a significant discrepancy between academic achievement and intellectual ability as assessed by professionals, i.e., a psychiatrist, pediatrician, or psychologist (Provincial Demonstration Schools, 2001). There are three English speaking Ontario Provincial Demonstration Schools: the Amethyst School in London, the Sagonaska School in Belleville, and the Trillium School in Milton.

There were three stages for defining the final four groups. Initially, secondary male school students (grades 9 to 12) were targeted in order to identify candidates for the DD group. However, it was found difficult to form chronological age-matched groups at this age range because very few secondary school students came forward to participate in the project. Subsequently, the target age range was lowered to recruit intermediate school students (grade 8) in addition to the inclusion of female students. A total of 137 students $($ males $=98$, females $=39)$ participated in the present project. Of these, 71 participants were selected to form four groups based on the grouping criteria: (1) a developmental dyslexic (DD) group, (2) a chronological age-matched (CA) group, (3) a chronological age-matched poor reader (CAP) group, and (4) a reading age-matched (RA) group. The stages are described in more detail in the next section. 
The sampling procedure was divided into three stages: (1) the initial sampling, (2) expanding the sample to a larger age range, and (3) the final grouping.

\subsubsection{Initial Sampling}

After ethical approval was obtained from the Carleton University Research Ethics Committee for Psychological Research in August 2002, a research proposal was submitted to the Ottawa-Carleton Research Advisory Committee, which is made up of representatives from two school boards in the Ottawa-Carleton region (i.e., OCDSB and OCCSB), to recruit the DD, CA, CAP and RA candidates. The recruitment criteria were: (1) for the DD group, male students in grades 9 to 11 who had severe reading difficulty but had average/above academic performance in at least one subject, such as math or science, (2) for the CA group, male students in grades 9 to 11 who were average/above average in both reading ability and academic performance, (3) for the CAP group, male students in grades 9 to 11 who had reading difficulty with generally poor academic performance, and (4) for the RA group, male students in grades 4 and 5, who were average/above average in both reading ability and academic performance. The OttawaCarleton Research Advisory Committee chose two Catholic secondary schools located in Ottawa, and granted the author permission to distribute a letter to parents (recruitment letter, see Appendix I) through school counselors on the condition that testing would take place outside school hours and off school property. The school counselors chose students based on the criteria submitted to the Research Advisory Committee. Approximately 200 letters to parents were distributed between these two secondary schools. Although a $\$ 20.00$ honorarium was offered as an incentive, only twelve students ( $7.9 \%$ of the final 
sample) with normal reading ability and average/above average academic performance came forward to participate in the project. At the same time as the submission to the Ottawa-Carleton Research Advisory Committee was made, contacts were made with the Learning Disabilities Association-Ottawa, a private school, learning centers in Ottawa and several DD students identified by professionals through the author's acquaintance. The private school turned down the request. No DD reader was identified through the learning centers. The Learning Disabilities Association-Ottawa, an organization of parents and professionals who are concerned about children and adults with learning disabilities, agreed to include a recruitment announcement (see Appendix J) in their monthly bulletin (January, February and June 2002). The criteria for recruitment were: (1) male secondary school students (age range of 14 to 18 years), and (2) with a diagnosis of LD in reading by professionals. Although a $\$ 20.00$ honorarium was offered as an incentive, only two students ( $1.3 \%$ of the final sample) with reading problems came forward to participate in the project. After three months, it was determined that this source could not provide enough participants. It was then decided to include female students and to recruit students with/without reading problems in grade 8 (age range of 12 to 13 years) from elementary schools.

\subsubsection{Expanding the Sample to a Larger Age Range}

The addition of female and younger students in the DD group required changes in the age of the RA group. The principals of two elementary schools in the OCDSB gave permission to test students in grades 3, 4 and 8 within school hours. Thus, the project ultimately accommodated elementary school students in grades 3 and 4 (age range of 8 to 
10 years) to form the RA group and students in grade 8 to form the CA, CAP and DD groups. One public school located in suburban Ottawa had an enrollment of 511 students, and provided French immersion, special education, and English as second language programs. Most students came from professional middle class homes. The other public school located in suburban Ottawa had an enrollment of 472 students, and provided special education including a learning disability class. Many students came from farming families.

In addition, contacts were made with the principals of the three Ontario Provincial Demonstration Schools to recruit DD students. All three English speaking schools granted permission to distribute recruitment letters to parents and to test students at their schools within school hours. The criteria for this recruitment were: (1) students in grades 8-10, and (2) students with a diagnosis of LD in reading by professionals. In total, 25 students from the Ontario Demonstration Schools participated in the project.

In total, 413 letters to parents were distributed to students through the two public elementary schools (110 letters), two Catholic secondary schools (200 letters), three Ontario Provincial Demonstration Schools (45 letters), the Learning Disabilities Association (50 letters), and the author's acquaintances ( 8 letters). The total number of positive and negative responses to the recruitment letter was $163(37.0 \%$ of the total distributed letters). The percentage of positive responses from each recruitment source was: (1) the elementary schools $(80.9 \%$ of the 110 letters $),(2)$ the secondary schools $(6.0$ $\%$ of the 200 letters), (3) the Provincial Demonstration Schools (96.9\% of the 45 letters), (4) the Learning Disabilities Association (4.0\% of the 50 letters), and (5) the author's acquaintances ( $62.5 \%$ of the 8 letters). One hundred and thirty-seven consents were 
obtained from parents through the schools, representing $93.2 \%$ of all responses. Ten parents ( $2.4 \%$ of the total distributed letters) did not give permission for their child's participation. The sources and distribution of the students are summarized in Table 9.1.

Table 9.1

List of Recruitment Sources and Distribution of Students

\begin{tabular}{|c|c|c|c|c|c|c|c|c|c|}
\hline \multirow[b]{2}{*}{ Names of sources } & \multicolumn{3}{|c|}{$\begin{array}{l}\text { Developmental dyslexic } \\
\text { group(DD) }\end{array}$} & \multicolumn{3}{|c|}{$\begin{array}{l}\text { Chronological age- } \\
\text { matched groups (CA \& } \\
\text { CAP) }\end{array}$} & \multicolumn{3}{|c|}{$\begin{array}{l}\text { Reading age-matched } \\
\text { group (RA) }\end{array}$} \\
\hline & $\mathbf{M}$ & $\mathbf{F}$ & Total & $\mathbf{M}$ & $\mathbf{F}$ & Total & $\mathbf{M}$ & $\mathbf{F}$ & Total \\
\hline $\begin{array}{l}\text { Ottawa-Carleton Catholic School Board } \\
\text { Notre Dame High-School } \\
\text { Immaculata High-School }\end{array}$ & $\begin{array}{l}0 \\
0\end{array}$ & $\begin{array}{l}0 \\
0\end{array}$ & $\begin{array}{l}0 \\
0\end{array}$ & $\begin{array}{l}10 \\
2\end{array}$ & $\begin{array}{l}0 \\
0\end{array}$ & $\begin{array}{l}10 \\
2\end{array}$ & $\begin{array}{l}0 \\
0\end{array}$ & $\begin{array}{l}\mathbf{0} \\
0\end{array}$ & $\begin{array}{l}0 \\
0\end{array}$ \\
\hline $\begin{array}{l}\text { Ottawa-Carleton District School Board } \\
\text { Henry Larsen Elementary School } \\
\text { Metcalfe Elementary School }\end{array}$ & $\begin{array}{l}2 \\
0\end{array}$ & $\begin{array}{l}0 \\
0\end{array}$ & $\begin{array}{l}2 \\
0\end{array}$ & $\begin{array}{l}21 \\
9\end{array}$ & $\begin{array}{l}11 \\
4\end{array}$ & $\begin{array}{l}32 \\
13\end{array}$ & $\begin{array}{l}24 \\
4\end{array}$ & $\begin{array}{l}12 \\
2\end{array}$ & $\begin{array}{l}36 \\
6\end{array}$ \\
\hline $\begin{array}{l}\text { Provincial Demonstration Schools } \\
\text { Amethyst School } \\
\text { Milton School } \\
\text { Sagonaska School }\end{array}$ & $\begin{array}{l}5 \\
1 \\
15\end{array}$ & $\begin{array}{l}4 \\
1 \\
2\end{array}$ & $\begin{array}{l}9 \\
2 \\
17\end{array}$ & $\begin{array}{l}1 \\
0 \\
0\end{array}$ & $\begin{array}{l}0 \\
0 \\
0\end{array}$ & $\begin{array}{l}1 \\
0 \\
0\end{array}$ & $\begin{array}{l}0 \\
0 \\
0\end{array}$ & $\begin{array}{l}0 \\
0 \\
0\end{array}$ & $\begin{array}{l}0 \\
0 \\
0\end{array}$ \\
\hline $\begin{array}{l}\text { Learning Disabilities Association- } \\
\text { Ottawa }\end{array}$ & 2 & 0 & 2 & 0 & 0 & 0 & 0 & 0 & 0 \\
\hline Acquaintances & 4 & 1 & 5 & 0 & 0 & 0 & 0 & 0 & 0 \\
\hline $\begin{array}{l}\text { Total } \\
153\end{array}$ & $\begin{array}{l}44 \\
28.8 \%\end{array}$ & $\begin{array}{l}8 \\
5.2 \%\end{array}$ & $\begin{array}{l}52 \\
34.0 \%\end{array}$ & $\begin{array}{l}44 \\
28.8 \%\end{array}$ & $\begin{array}{l}15 \\
9.8 \%\end{array}$ & $\begin{array}{l}59 \\
38.5 \%\end{array}$ & $\begin{array}{l}28 \\
18.3 \%\end{array}$ & $\begin{array}{l}14 \\
9.1 \%\end{array}$ & $\begin{array}{l}42 \\
27.4 \%\end{array}$ \\
\hline $\begin{array}{ll}\text { Total participants } & =137(37.1 \%) \\
\text { Total refusals } & =10(2.4 \%) \\
\text { No response } & =266(60.5 \%) \\
\text { Total distributed letters } & =413\end{array}$ & & & & & & & & & \\
\hline
\end{tabular}

\subsubsection{Final Grouping}

As part of the testing protocol, measurements of non-verbal IQ and reading ability were included in the test battery. These measures were used to screen and to group the sample to form final groups. All participants were given the following tests: (1) the standardized reading test (Test of Word Reading Efficiency, TWORE, Torgesen, Wagner, \& Rashotte, 1999) (Appendix F); (2) the non-standardized vocabulary test (Castle \& Coltheart, 1993) (Appendix G); and (3) the standardized non-verbal test (Test of Nonverbal Intelligence Test-3, TONI-3, Brown, Sherbenou, \& Johnson, 1980) 
(Appendix H). Two questionnaires were used to collect additional information: (1) a Parent Questionnaire (Appendix I), and (2) a Student Questionnaire (see Appendix K). Parents were asked to fill out a consent form and return it to their child's school with the Parent Questionnaire. In the Parent Questionnaire, parents were asked to rate their children on a five-point scale in the following areas: (a) access to educational opportunities, (b) current reading skills, (c) current academic achievement in math and science, (d) past head injuries, (e) past or current diagnosis with AD/HD or LD, and (f) current general health including hearing and vision. The information from the questionnaires was important because it provided additional information as to whether a student's reading problem stemmed from factors other than DD. In the Student Questionnaire, students rated themselves on a five-point scale in two areas: (1) their fondness for reading, and (2) the amount of time spent on reading in addition to school work per day.

The information from parents and students was used to categorize the participants into four groups: (1) a developmental dyslexic (DD) group, (2) a chronological agematched (CA) group, (3) a chronological age-matched poor reader (CAP) group, and (4) a reading age-matched (RA) group. The criteria for the DD group were as follow: (1) diagnosis of a reading disability or $L D$ in reading by a professional as reported on the Parent Questionnaire; (2) a standardized score (SS) of 95 or above $\left(36^{\text {th }}\right.$ percentile for age) based on the TONI-3 (Test of Non-Verbal Intelligence); (3) an SS of 81 or below $\left(10^{\text {th }}\right.$ percentile for age) based on the TOWRE (Test of Word Reading Efficiency), (4) no reported diagnosis of $\mathrm{AD} / \mathrm{HD}$ deficits or other neurological disorders based on the Parent Questionnaire; (5) no reported experience of head injury; (6) no reported visual/hearing 
impairment; and (7) a rating of 2 or above on the five-point scale of educational opportunities based on the Parent Questionnaire. In order to sample those DD students who were severely dyslexic but maintained intact non-verbal abilities, the cut-off point was set above $1 S D$ (i.e., brighter than average) for the TONI-3 and below $3 S D$ (i.e., severe reading difficulty) for the TOWRE. This large discrepancy between non-verbal intelligence and reading ability would sample those who fit the definition of DD with unexpected reading difficulties. For the CA and RA groups, the criteria were: (1) an SS of 95 or above in the TONI-3 to accord with the DD group, and (2) an SS of 90 or above $\left(25^{\text {th }}\right.$ percentile for age) in the TOWRE as normal readers. For the CAP group, the criteria were: (1) an SS of 85 or below ( $15^{\text {th }}$ percentile for age) in the TONI-3, and (2) an SS of 90 or below in the TOWRE.

The final sample consisted of: (1) 19 students (15 males, 4 females) identified as having DD (mean age of 13.08 years), (2) 25 students (20 males, 5 females) in the CA group (mean age of 13.04 years), (3) 10 students ( 8 males, 2 females) in the CAP group (mean age of 13.09 years), and (4) 17 students (14 males, 4 females) in the RA group (mean age of 9.01 years). Unexpectedly, the young age participants in the RA group (mean age of reading $=12.06$ years) turned out to be far better readers than those in the DD group (mean reading age $=8.02$ years), and as a result, this group was subsequently renamed the young age (YA) group. The CA group also turned out to be above average readers (mean reading age of 16.01 years).

Students excluded from the DD group were those who had: (1) parental reports of attentional deficits (13 students), (2) low non-verbal IQ (9 students), (3) reading ability above the criterion ( 3 student), (4) a young age ( 5 students, 11.0 years), or (5) declined to 
participate ( 3 students). From the CA group, 18 students were excluded due to their age (mean age of 11 years), and 10 were excluded due to their low non-verbal IQ. From the YA group, eight students were excluded due to low non-verbal IQ, and eight students due to age (mean age of 7.5 years), and four students declined to participate. In total, 66 students were excluded from the sample. A profile of each group is summarized in Table 9.2 .

Table 9.2

Profile of an Experimental Group and Control Groups

\begin{tabular}{|c|c|c|c|c|c|c|}
\hline \multirow[b]{2}{*}{ Group } & \multirow[b]{2}{*}{ Participants } & & \multicolumn{4}{|c|}{ Assessments } \\
\hline & & $\begin{array}{l}\text { Chronological Age } \\
\text { (Mean of age in year) }\end{array}$ & $\begin{array}{l}\text { TONI-3 } \\
\text { SS } \\
\text { (Mean) }\end{array}$ & $\begin{array}{l}\text { TOWRE } \\
\text { SS } \\
\text { (Mean) }\end{array}$ & $\begin{array}{c}\text { TOWRE Reading } \\
\text { Age } \\
\text { (Mean age in year) }\end{array}$ & $\begin{array}{c}\text { Handedness } \\
\text { LQ } \\
\text { (Mean) }\end{array}$ \\
\hline$\overline{\mathrm{CA}}$ & $\begin{array}{l}\mathrm{n}=25 \\
\text { male } 20 \\
\text { female } 5\end{array}$ & $\begin{array}{c}13.04 \\
(S D=.68) \\
\text { Age range } \\
12.01-15.05\end{array}$ & $\begin{array}{c}102.6 \\
(S D=8.21)\end{array}$ & $\begin{array}{c}112 \\
(S D=10.77)\end{array}$ & $\begin{array}{c}16.01 \\
(S D=1.41)\end{array}$ & $\begin{array}{c}+0.75 \\
(S D=.14)\end{array}$ \\
\hline CAP & $\begin{array}{ll}n= & 10 \\
\text { male } & 8 \\
\text { female } & 2\end{array}$ & $\begin{array}{c}13.09 \\
(S D=1.14) \\
\text { Age range } \\
13.01-16.08\end{array}$ & $\begin{array}{c}83.8 \\
(S D=4.29)\end{array}$ & $\begin{array}{c}84.5 \\
(S D=6.57)\end{array}$ & $\begin{array}{c}11.0 \\
(S D=2.15)\end{array}$ & $\begin{array}{c}+0.63 \\
(S D=.34)\end{array}$ \\
\hline DD & $\begin{array}{c}n= \\
\begin{array}{c}\text { male } \\
\text { female }\end{array} \\
4\end{array}$ & $\begin{array}{c}13.08 \\
(S D=1.63) \\
\text { Age range } \\
12.11-18.08\end{array}$ & $\begin{array}{c}102.7 \\
(S D=9.17)\end{array}$ & $\begin{array}{c}69.2 \\
(S D=6.85)\end{array}$ & $\begin{array}{c}8.02 \\
(S D=0.84)\end{array}$ & $\begin{array}{c}+0.50 \\
(S D=.50)\end{array}$ \\
\hline YA & 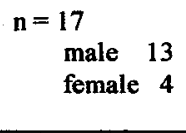 & $\begin{array}{c}9.01 \\
(S D=.68) \\
\text { Age range } \\
8.04-10.08\end{array}$ & $\begin{array}{c}105.0 \\
(S D=14.43)\end{array}$ & $\begin{array}{c}113.9 \\
(S D=14.23)\end{array}$ & $\begin{array}{c}12.06 \\
(S D=2.93)\end{array}$ & $\begin{array}{c}+0.72 \\
(S D=.33)\end{array}$ \\
\hline Total & $\begin{array}{cc}\mathrm{N}=71 \\
\text { male } & 56 \\
\text { female } & 15\end{array}$ & & & & & \\
\hline
\end{tabular}

Note: $\mathrm{CA}=$ Chronological age-matched normal readers; CAP = Chronological age-matched poor readers;

$\mathrm{DD}=$ Developmental dyslexic students; YA = Young age readers; $S D=$ standard deviation; $\mathrm{SS}=$ Standardized Score;

In Handedness column, $+=$ right hand preference.

\subsection{Handedness}

Handedness was assessed using the 10 handedness items from the Edinburgh Handedness Inventory (Oldfield, 1971); (see Appendix L). Each item is a unimanual task (e.g., writing, drawing, throwing, and using scissors) through which participants indicate 
strength of preference as "+/++ (right hand preference)" or "-1--(left hand preference)". This inventory provides a laterality quotient (LQ) that ranges from +1.0 to -1.0 based on the following formula: $\mathrm{LQ}=$ (total right hand - total left-hand) $/$ (total right hand + total left hand). The participants were categorized as either "consistent" right-handed (CRH) or "non-consistent" right-handed (nCRH). The $\mathrm{nCRH}$ included three left-handed students (i.e., LQ is represented in negative values) using a cut-off point of +.75 (Habib et al., 1991; Robichon \& Habib, 1998). The group means were as follows: +.75 for the CA group, +.63 for the CAP group, +.50 for the DD group, and +.72 for the YA group.

\subsection{Verbal and Non-Verbal Assessment Materials}

To obtain measures of non-verbal IQ and reading ability, all participants were given the following tests: (1) a standardized reading test (Test of Word Reading Efficiency, TWORE, Torgesen, Wagner, \& Rashotte, 1999) (Appendix F), (2) a nonstandardized vocabulary test (Castle \& Coltheart, 1993) (Appendix G), and (3) a standardized non-verbal test (Test of Nonverbal Intelligence Test-3, TONI-3, Brown, Sherbenou, \& Johnson, 1980) (Appendix H).

\subsubsection{Verbal Tests}

Two verbal tests were administered to all participants to assess their reading ability: (1) a standardized reading test, TOWRE (Torgesen et al., 1999), (Appendix F), and (2) a non-standardized vocabulary test (Castle \& Coltheart, 1993), (Appendix G). The TOWRE contains two sub-tests: the Sight Word Efficiency (SWE) and the Phonemic Decoding Efficiency (PDE). The SWE measures the number of real printed 
words that can be accurately identified within 45 seconds, whereas the PDE measures the number of pronounceable printed nonwords that can be accurately decoded within 45 seconds. The TOWRE was used for four purposes: (1) to confirm that the DD children had a reading disability that was at least two grades behind the CA group, (2) to test two components of reading ability: sight reading and phonological decoding, (3) to consider fluency in reading ability (i.e., timed test), and (4) to benefit from the short administration time (i.e., 45 seconds).

The reliability of the TOWRE was also investigated by Torgesen, et al. (1999): (1) for internal consistency, which requires that all items in a test measuring the same qualities, the correlation coefficient was .96 between Form A and Form B; (2) for testretest reliability, the correlation coefficient was .97 between the first and second tests administered within a week; and (3) for examiner reliability, the correlation coefficient was .99 between the two examiners. These correlation coefficients indicate that the overall reliability of the TOWRE is strong.

The validity of the TOWRE was investigated by Torgesen, et al. (1999): (1) for content validity, which refers to "whether a test covers a representative sample of the behaviour domain to be measured " (Anastasi \& Urbina, 1997, p. 114-115), the discrimination index (a correlation coefficient that represents a relationship between a particular item and the other items on the test) for Forms A and B in both SWE (Sight Word Efficiency) and PDE (Phonological Decoding Efficiency) was .53 on average, and exceeded the suggested discrimination index of .35 , and the item difficulty, which is determined to identify items that are too easy or too difficult, exceeded the satisfactory index of .50; (2) for concurrent validity, the correlation between the PDE and the Word 
Attack subtest of the Woodcock Reading Mastery Tests-Revised (WRMT-R);

(Woodcock, 1987) was .85; and the SWE and the Word Identification subtest of the WRMT-R was .89; and (3) for predictive validity, the SWE predicted reading rate $(r=$ $.80)$, accuracy $(r=.80)$, and comprehension $(r=.80)$ in the $4^{\text {th }}$ and $5^{\text {th }}$ grades; and the PDE also predicted reading rate $(r=.47)$, accuracy $(r=.68)$, and comprehension $(r=$ .66) using the Gray Oral Reading Tests (Wiederholt \& Bryant, 1992) with severe reading disabilities in the $4^{\text {th }}$ and $5^{\text {th }}$ grades. The correlations described above indicate that the overall validity of the TOWRE is strong.

The non-standardized vocabulary test contains 30 regular, 30 irregular and 30 pronounceable nonwords. This vocabulary test was included to see if there were subtypes in the DD groups. This test has been used in a number of studies of DD to distinguish sub-types (Castles \& Coltheart, 1993; Coltheart \& Leahy, 1996; Temple, 1984).

\subsubsection{Non-Verbal Test}

The Test of Nonverbal Intelligence-3 (TONI-3) (Brown et al., 1980) was administered to ensure that the DD group had similar intelligence to the CA and YA groups, and that the CAP group had lower intelligence than other groups in non-verbal intelligence (Appendix H). The TONI-3 is a standardized, language-free non-verbal intelligence test concentrating on problem solving and hypothetical mental ability (Brown et al., 1980). Since this is a language-free test, the examinee's task is simply to point to an appropriate drawing out of four or six choices, which logically corresponds to a set of presented drawings, without using verbal communication. 
The reliability of the TONI-3 was reported as follows: (1) the correlation coefficient for the internal consistency, was 0.93 , (2) the correlation coefficient for the test-retest reliability was 0.92 , and (3) the correlation coefficient for the examiner differences was 0.99 (Brown et al., 1999). These correlation coefficients indicate that the reliability of the TONI-3 is strong.

Brown, et al. (1999) investigated a number of forms of validity: (1) for content validity, the discrimination index for Form $A$ and $B$ was .49 on average, which exceeded the suggested criterion of .35 , and the item difficulty was $65 \%$ on average, which exceeded the satisfactory level of $50 \%$; (2) for concurrent validity, the TONI-3 was highly correlated with the Comprehensive Test of Nonverbal Intelligence (Hammill, Pearson, \& Wiederholt, 1996) ( $r=.76$ for Form A, $r=.74$ for Form B), with the Wechsler Intelligence Scale for Children-Third Edition (WISC-III) (Wechsler, 1991) $(r=$ 63 for Form A and B), and the Wechsler Adult Intelligence Scale-Revised (WAIS-R) (Wechsler, 1981) $(r=.73$ for Form A, $r=.71$, for Form B); and (3) for predictive validity, the correlation coefficients with writing comprehension, broad reading, mathematics and knowledge were in the range of .55 to .76 . These correlation coefficients indicate that the reliability of the TONI-3 is strong.

\subsection{Confirming Groupings}

A series of one-way Analysis of Variance (ANOVA) was performed to confirm that these four groups differed as expected on the following variables: Age, non-verbal IQ and reading scores. Regarding Age, there was a significant effect of group, $F(3,68)=$ $81.0, p<.000, \mathrm{y}^{2}=.781$. The Bonferroni correction was used for Pairwise comparisons 
to adjust $\alpha$ level because this approach ensured avoiding Type I errors (Stevens, 1996). The pairwise comparisons revealed that there was a significant difference between the YA group and the other three groups (DD, CA and CAP), $p<.000$, but no difference among these groups. Regarding TONI-3, there was a significant effect of group, $F(3,68)$ $=11.04, p<.000, \mathrm{n}^{2}=.328$. The pairwise comparisons revealed that the CAP group significantly differed from the other three groups (DD, CAP and YA), $p<.000$, but there was no difference among these groups. Regarding TOWRE, there was a significant effect of group, $F(3,68)=82.2, p<.000, \mathrm{n}^{2}=.784$. The pairwise comparisons revealed that the DD group significantly differed from the other three groups (CA, CAP and YA), $p<$ .000 ; the CA group differed from the $\mathrm{DD}$ and CAP groups but not from the YA group, $p$ $<.000$; the CAP group differed from the other groups (DD, CAP, and YA), $p<.000$; and the YA group differed from the DD and CAP groups, $p<.000$, but not from the CA group. The data are summarized in Figure 9.1. The analyses confirmed that all groups were categorized as expected. That is, in terms of reading ability, the DD group differed from all other groups, and specifically, they were worse performers than the CAP group. In terms of non-verbal intelligence, the DD group was better than the CAP group, and had similar ability to the CA and YA groups. This ensured that the DD group had reading difficulty with high non-verbal intelligence. Furthermore, the CAP group showed poorer abilities in both tests than the CA and YA groups. This ensured that the CAP group had reading difficulty with low non-verbal intelligence. 


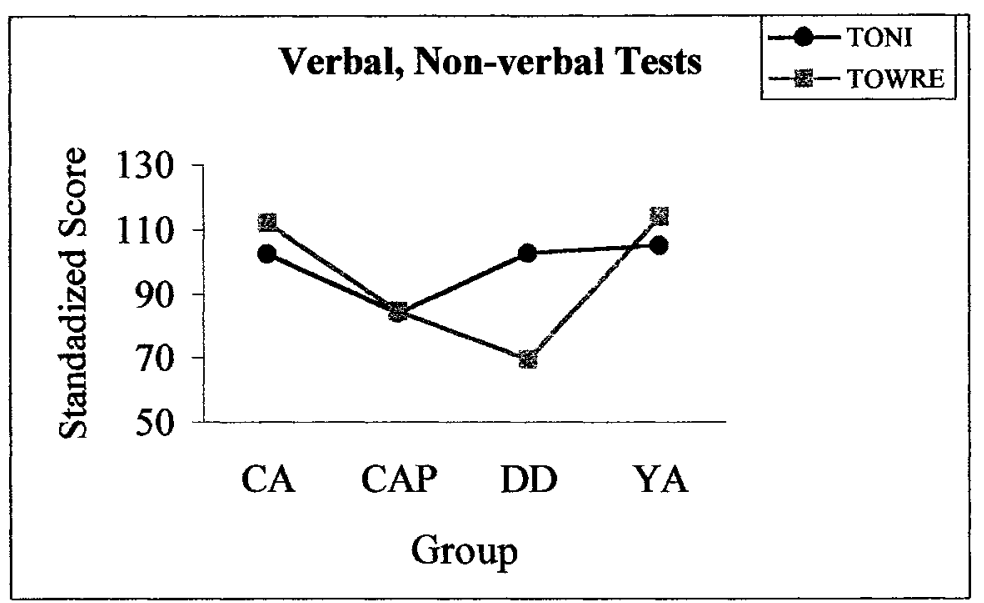

Figure 9.1. The results of the Test of Word Reading Efficiency (TOWRE)

and the Test of Nonverbal Intelligence-3 (TONI-3) in the four groups.

$\mathrm{CA}=$ Chronological age-matched normal readers

$\mathrm{CAP}=$ Chronological age-matched poor readers.

$\mathrm{DD}=$ Developmental dyslexic students.

$\mathrm{YA}=$ Young age readers.

\subsection{Investigation of Sub-types of Developmental Dyslexia}

As discussed in Chapter 2.4, there is the possibility of the existence of sub-types of DD (Boder, 1971, 1973; Castle and Coltheart, 1993; Coltheart et al., 1983; Ellis, 1994; Temple, 1984). Although the different researchers have identified sub-types of DD by different terminology, they all identify similar groups as involving problems either with phonological processing (i.e., phonological dyslexia or dysphonesia) or with visualorthographic processing (surface dyslexia or dyseidesia), or both (mixed dyslexia or dysphoneidesia). To investigate the existence of sub-types of DD in the present study, an examination was made of the individual reading scores on the non-standardized vocabulary test (Castle \& Coltheart, 1993) (see Appendix G) by each DD student. Castle 
and Coltheart (1993) defined surface dyslexia as the existence of deficits in visualorthographic processing that result in problems in reading irregular words, but that cause few problems in reading regular words and nonwords. They also defined phonological dyslexia as the existence of deficits in phonological processing that result in problems in reading regular words and nonwords, but cause few problems in reading irregular words. Thus, the vocabulary test scores of regular and irregular words and nonwords for each DD student were investigated to see if they would fall within $2 S D$ of the group mean of the YA group, which is considered a normal range. The comparison was made between the DD and YA groups because the mean reading age of the CA group (16.01 years) in the present study exceeded their chronological age (13.04 years). Because the mean reading age of the YA group (mean reading age of 12.01 years) was closest to the chronological age of the DD group (13.08 years), it was decided to use their scores to calculate the $2 S D$ of normal distribution of reading ability in 13-year-old students.

With respect to irregular words, 14 out of $19 \mathrm{DD}$ students had irregular reading scores below $2 S D$ in the sample distribution. Among the 14 , one student showed normal regular and nonword reading scores. Thus, this student was identified as having surface dyslexia, i.e., deficits in visual-orthographic processing with normal phonological processing. With respect to regular word and nonword reading, 16 out of 19 DD students had reading scores below $2 S D$ in the sample distribution. Of these 16 students, all but two demonstrated irregular reading scores below $2 S D$ in the distribution. As a result, 14 DD students were identified as having mixed dyslexia, i.e., deficits in both phonological and visual-orthographic processing. The two DD students were identified as normal 
readers by this vocabulary test. However, the sub-tests of the TOWRE scores (i.e., SWE and PDE) showed that their scores were below $2 S D$ in the standardized distribution.

It was concluded that there was one possible surface dyslexic student, but the rest of the students in this sample were identified as having mixed dyslexia. 


\section{Chapter 10: Experiment 1}

The aim of Experiment 1 was to investigate the involvement of inefficient interhemispheric information transfer in DD using a finger localization task associated with the posterior midbody of the CC (corresponding to the CC \# 5 in Figure 5.2). The detailed rationale for Experiment 1 was discussed in Chapter 8.3.1. Three points, however, should be emphasized prior to formulating specific predictions related to the interhemispheric inefficiency hypothesis. First, callosal information transfer is associated with a small but reliable degradation of information (Geffen et al., 1985). Second, developmental studies suggest that there is a positive correlation between age and the development of callosal efficiency in tactile information (Galin et al., 1977; Galin et al., 1979; Quinn \& Geffen, 1986). Third, previous research (Fletcher et al., 1982; Lindgren, 1978; Moore et al., 1996; Zung, 1986) found that scores in the Crossed condition correlated with reading ability. Based on these points, the following predictions were formulated:

Prediction 1: All groups would show lower finger localization scores in the Crossed than in the Uncrossed condition due to callosal information transfer.

Prediction 2: The CA group would have the highest scores, the YA and CAP groups would show similar scores, and the DD group would have the lowest scores in the Crossed condition due to a combination of age and reading ability. 
Prediction 3: The DD group would show a larger difference in the Crossed-UncrossedDifference (CUD) scores compared to the CA and CAP groups due to impairments in the $\mathrm{CC}$.

Prediction 4: The YA group would show a larger difference in the CUD scores compared to the CA and CAP groups due to immature callosal development.

Prediction 5: In the Uncrossed condition, all four groups would show similar performance in the 2-Finger Sequence due to the easiness of the task and the lack of interhemispheric communication.

Prediction 6: The DD group would make more sequence errors than the other groups due to their poor sequence processing (e.g., Bakker, 1977).

Prediction 7: Standardized reading scores (SS) would be positively correlated with task performance in the Crossed condition as predicted by the interhemispheric disconnection theory.

Prediction 8: If DD is qualitatively distinct from poor reading ability, the DD group would show different patterns of performance from the CAP group.

\subsection{Method}

\subsubsection{Finger Localization Task Protocol}

The finger sequence task consisted of 2-Finger, 3-Finger, and 4-Finger Sequence. These tasks were tested under four conditions: right and left hand stimulation by two response modes involving a response with the same hand, or a response with the opposite hand. Thirty trials were generated for each condition, i.e., each finger sequence contained 
10 trials. The three types of the finger sequences were randomized in four conditions, but the presentation of more than three consecutive same finger sequences in each condition was avoided. The total number of the trials for each participant was 120 . The finger sequence protocol is reproduced in Appendix $M$.

Two small soft cloth cushions $(7 \times 5 \mathrm{~cm})$ and a wooden screen $(60 \times 25 \mathrm{~cm})$ with a square opening through which a participant could place both hands were used. A crochet needle $(12 \mathrm{~cm}$ in length) was used to stimulate the fingers.

\subsubsection{Procedure}

All participants were individually tested. Most were tested in a quiet small room at their school and a small number of participants were tested in a laboratory setting at Carleton University. The participant was seated opposite the experimenter. Visual feedback was eliminated using a wooden screen. The hands were maintained in a "palm down" position. A crochet needle was used to lightly press the tips of the fingers. The task was to reconstruct the finger sequences following stimulating of the fingers. There were four conditions based on the hand stimulated by the responding hand: right or left hand stimulated and response with the same hand (Uncrossed condition), and right or left hand stimulated and response with the opposite hand (Crossed condition). That is, in the Uncrossed condition, the participant was required to reconstruct the finger sequence by touching each finger in the sequence with the thumb of the same hand. In the Crossed condition, the participant was required to reconstruct the finger sequence by touching corresponding fingers with the thumb of the non-stimulated hand. The participant was instructed to respond as soon as the experimenter said 'go'. The types of finger sequences 
were explained and demonstrated to the participant, i.e., 2-, 3-, and 4-finger sequences were randomly touched. To avoid the participant's expectation as to which finger would be touched next, the participant was also told that the same finger might be touched twice in a trial. It was also stressed that the participant should avoid extra finger movements during the test and to respond in the same order as in the stimulation. Three practice trials were given at the beginning in which the right hand and left hand were stimulated. Feedback was provided when a participant made an incorrect response in the practice trials but was not provided in the experimental trials. The test trials always started with a 2-Fnger Sequence because it was the easiest. The order of the stimulated hand (right/left) and condition (Uncrossed/Crossed) were counterbalanced; however, the same hand was sequentially stimulated. That is, one quarter of the participants started with the right hand in the Uncrossed condition followed by the same hand in the Crossed condition, one quarter started with right hand in the Crossed condition followed by the same hand in the Uncrossed condition, one quarter started with the left hand in the Uncrossed condition followed by the same hand in the Crossed condition, and one quarter started with the left hand in the Crossed condition followed by the Uncrossed condition.

\subsubsection{Analysis}

The number of correct responses and errors recorded in each condition were translated into percentages for the analysis to facilitate comparison with previous studies using percentage data (Geffen et al., 1985; Gross-Glenn \& Rothenberg, 1984; Lindgren, 1978; Quinn \& Geffen, 1986). The CUD (Crossed-Uncrossed-Difference) was calculated by subtracting the correct percentages in the Crossed condition from those in the 
Uncrossed condition. When main or interaction effects were observed, the Bonferroni corrected $\alpha$ (i.e., $\alpha=.05$ was divided by the number of comparisons) was applied for post hoc tests to ensure avoiding Type I errors (Stevens, 1996).

To test Predictions 1, 2, 3, and 4, a 4 (Group: CA, CAP, DD, YA) x 2 (Condition: Uncrossed, Crossed) $\times 3$ (Finger Sequence: two, three, four) $\times 2$ (Hand: right, left) ANOVA with mixed repeated-measures was used. Group was entered into the analysis as a between-subjects factor, and Condition, Finger Sequence, and Hand were entered into the analysis as within-subjects factors. The dependent variable (DV) was the percentage of correct responses.

To test Predictions 5 and 6, the sequence errors were calculated and entered into the analysis. The sequence errors were errors in which correct fingers were indicated, but in the wrong order. Sequence errors were analyzed by a 4 (Group: CA, CAP, DD, YA) $x$ 2 (Condition: Uncrossed, Crossed) $\times 2$ (Sequence Error: one, two) $\times 2$ (Hand: right, left) ANOVA with mixed repeated-measures. Group was entered into the analysis as a between-subjects factor, and Condition, Sequence Error, and Hand were entered into the analysis as within-subjects factors. The DV was the percentage of sequence errors.

To test Prediction 7, a correlation analysis was conducted on the percentage of correct responses in the 2- and 3-finger sequence on the Uncrossed and Crossed conditions and age, standardized reading scores (TOWRE) and standardized non-verbal IQ scores. 


\subsection{Results}

A preliminary analysis was conducted in order to investigate the following: (1) the presence of ceiling and floor effects, (2) the presence of extreme values (i.e., values exceeding $3 S D$ ) in each group (Stevens, 1996), (3) the normal distribution of each group, and (4) the homogeneity of variances for the groups. The four investigations were important for a variety of reasons. The first investigation was significant because the observations on the DV were expected to be distributed around the mean or median in a normal distribution (Howell, 1987). However, the presence of ceiling and floor effects indicated that observations were not distributed as expected (Howell, 1987).

The second investigation was important because extreme values can affect the statistical analysis (Stevens, 1996). A Box plot test was conducted on each observation on the DV to detect extreme values in each group (Stevens, 1996). The Box plot represents the distribution of observations. A box in the plot showing a large size indicates a reasonably good distribution of each observation of the data. The Box plot test identifies values exceeding $3 S D$ on the plot. With regard to this experiment, no extreme value was detected in the data. The third and fourth investigations were significant because ANOVA assumes normal distribution within each group and homogeneous variance in the populations (Stevens, 1996). The Shapiro-Wilk test for normality and the Levene test for the homogeneity of variance were performed in each analysis. No violation of these assumptions was revealed by the tests.

The group means of the percentages of correct responses and sequence errors are presented in Table 10.1 and 10.2, respectively. An ANOVA was also performed to 
examine group differences on Gender and Handedness in each group. The analyses

revealed no significant group differences in these variables. These data were

subsequently pooled across Gender and Handedness.

Table 10.1.

Mean Percentage Correct Responses and Sequence Errors for Four Groups

\begin{tabular}{|c|c|c|c|c|c|}
\hline & & \multicolumn{4}{|c|}{ Correct Responses } \\
\hline \multirow[b]{3}{*}{ Group } & \multirow[b]{3}{*}{$\begin{array}{c}\text { Finger } \\
\text { Sequence }\end{array}$} & \multicolumn{4}{|c|}{ Condition Mean $\%(S D)$} \\
\hline & & \multicolumn{3}{|c|}{ Left Hand } & \multirow{2}{*}{$\frac{\text { Right Hand }}{\text { Crossed }}$} \\
\hline & & Uncrossed & Crossed & Uncrossed & \\
\hline \multirow[t]{3}{*}{$\overline{\mathrm{DD}}$} & 2 & $72.63(16.61)$ & $54.21(19.80)$ & $73.68(17.70)$ & $52.11(18.13)$ \\
\hline & 3 & $60.52(19.85)$ & $43.16(16.00)$ & $61.58(18.33)$ & $38.32(21.67)$ \\
\hline & 4 & $32.11(18.43)$ & $18.95(11.49)$ & $34.73(20.38)$ & $20.00(14.53)$ \\
\hline \multirow[t]{3}{*}{$\mathrm{CA}$} & 2 & $87.60(13.63)$ & $74.40(21.62)$ & $93.60(8.62)$ & $76.40(20.99)$ \\
\hline & 3 & $78.80(20.88)$ & $62.00(23.09)$ & $80.00(16.07)$ & $54.40(26.78)$ \\
\hline & 4 & $70.40(23.00)$ & $48.16(16.00)$ & $70.80(21.39)$ & $40.00(25.82)$ \\
\hline \multirow[t]{3}{*}{ CAP } & 2 & $83.00(18.29)$ & $59.00(21.32)$ & $80.00(18.86)$ & $56.00(22.71)$ \\
\hline & 3 & $71.00(16.87)$ & $40.00(20.00)$ & $72.00(18.74)$ & $44.00(18.38)$ \\
\hline & 4 & $53.00(33.02)$ & $20.00(12,47)$ & $46.00(27.16)$ & $19.00(18.53)$ \\
\hline \multirow[t]{3}{*}{ YA } & 2 & $74.12(12.78)$ & $55.29(20.35)$ & $70.00(20.31)$ & $54.12(20.93)$ \\
\hline & 3 & $57.65(27.05)$ & $35.88(23.20)$ & $64.71(24.01)$ & $35.29(25.77)$ \\
\hline & 4 & $32.35(25.38)$ & $11.76(14.68)$ & $32.94(23.92)$ & $20.59(25.37)$ \\
\hline
\end{tabular}

Note: $S D=$ Standard deviation; $\mathrm{DD}=$ developmental dyslexic;

$\mathrm{CA}=$ Chronological age-matched; $\mathrm{CAP}=$ Chronological age-matched poor readers;

$\mathrm{YA}=$ Young age

Table 10.2.

Mean Percentage of Sequence Errors for Four Groups

\begin{tabular}{|c|c|c|c|c|c|}
\hline \multirow[b]{4}{*}{ Group } & \multicolumn{5}{|c|}{ Correct Responses } \\
\hline & \multirow[b]{3}{*}{$\begin{array}{c}\text { Finger } \\
\text { Sequence }\end{array}$} & \multicolumn{4}{|c|}{ Condition Mean $\%(S D)$} \\
\hline & & \multicolumn{2}{|c|}{ Left Hand } & \multicolumn{2}{|c|}{ Right Hand } \\
\hline & & Uncrossed & Crossed & Uncrossed & Crossed \\
\hline \multirow[t]{3}{*}{ DD } & 2 & $2.63(6.53)$ & $4.74(5.13)$ & $1.05(4.59)$ & $9.47(9.70)$ \\
\hline & 3 & $7.37(9.33)$ & $15.79(12.16)$ & $9.47(9.70)$ & $13.68(10.65)$ \\
\hline & 4 & $17.37(15.58)$ & $21.58(13.44)$ & $12.63(9.33)$ & $20.00(14.14)$ \\
\hline \multirow[t]{3}{*}{$\mathrm{CA}$} & 2 & $0.80(2.77)$ & $6.40(7.57)$ & $0.80(2.77)$ & $7.20(12.75)$ \\
\hline & 3 & $3.60(6.38)$ & $7.60(10.91)$ & $3.60(7.57)$ & $10.40(9.35)$ \\
\hline & 4 & $11.20(13.94)$ & $19.20(11.87)$ & $11.20(10.92)$ & $23.20(18.87)$ \\
\hline \multirow[t]{3}{*}{ CAP } & 2 & $2.00(4.22)$ & $15.00(18.41)$ & $0.00(0.00)$ & $10.00(9.43)$ \\
\hline & 3 & $5.00(7.07)$ & $15.00(19.00)$ & $7.00(8.23)$ & $10.00(9.43)$ \\
\hline & 4 & $16.00(14.30)$ & $28.00(21.50)$ & $18.99(16.87)$ & $28.00(16.19)$ \\
\hline \multirow[t]{3}{*}{ YA } & 2 & $1.18(3.32)$ & $5.88(7.12)$ & $1.18(3.32)$ & $10.59(9.66)$ \\
\hline & 3 & $4.12(7.12)$ & $16.47(11.69)$ & $6.47(8.62)$ & $15.88(10.04)$ \\
\hline & 4 & $5.88(7.12)$ & $17.06(13.59)$ & $10.59(14.78)$ & $19.41(13.45)$ \\
\hline
\end{tabular}

Note: $S D=$ Standard deviation; DD: Developmental dyslexic;

$\mathrm{CA}=$ Chronological age-matched; $\mathrm{CAP}=$ Chronological age-matched poor readers;

$\mathrm{YA}=$ Young age 
The primary analysis revealed that there was no main and interaction effect of Hand, $F(1,67)=.071, p=.790, \mathrm{y}^{2}=.001$. This indicated that this independent variable did not have any impact on the dependent variable. The data were then reanalyzed, collapsing across the left and right hands. The analysis revealed that there were main effects of Group, $F(1,3)=23.37, p<.000, \mathrm{n}^{2}=1.000$, Condition, $F(3,138)=290.16, p$ $<.000, \mathrm{y}^{2}=.171$, and Finger Sequence, $F(2,137)=328.61, p<.000, \mathrm{\eta}^{2}=.828$. There was also a three-way interaction of Condition $x$ Finger Sequence $x$ Group, $F(6,274)=$ $2.15, p<.048, \mathrm{\eta}^{2}=.045$.

As expected, the CA group $(69.77 \%, S D=20.6)$ demonstrated the most accurate performance among the four groups, $p=.000$, followed by the CAP group $(M=53.67 \%$, $S D=20.5)$, the DD group $(M=46.84 \%, S D=17.77)$, and the YA group $(M=45.39 \%$, $S D=22.21)$. The DD, CAP and YA groups did not significantly differ from each other. Regarding Condition, overall, the finger localization was more accurate in the Uncrossed $(M=64.76 \%, S D=19.60)$ than the Crossed $(M=43.07 \%, S D=20.12)$ Condition, $p<$ .000 , across the four groups. Regarding Finger Sequence, the 2-finger sequence $(M=$ $69.76 \%, S D=19.85)$ was responded to more accurately than the 3 -finger sequence $(M=$ $56.28 \%, S D=21.07)$ and the 4-finger sequence $(M=35.72 \%, S D=21.44)$ across the Conditions.

Regarding the three-way interaction of Condition $x$ Finger Sequence $x$ Group, pairwise comparisons revealed that in the 2-Finger and 3-Finger Sequences, the DD and YA groups responded significantly less accurately than the CA group in the Uncrossed condition, $p<.000$, but these groups did not significantly differ from each other. In the 4-Finger Sequence, the DD, CAP, and YA groups demonstrated significantly less 
accurate responses than the CA group in the Uncrossed condition. With respect to the Crossed condition, the DD, CAP, and YA groups significantly differed from the CA group in the 2-Finger, 3-Finger and 4-Finger Sequences, $p<.001$, but these groups did not significantly differ from each other. The data are summarized in Figure 10.1.
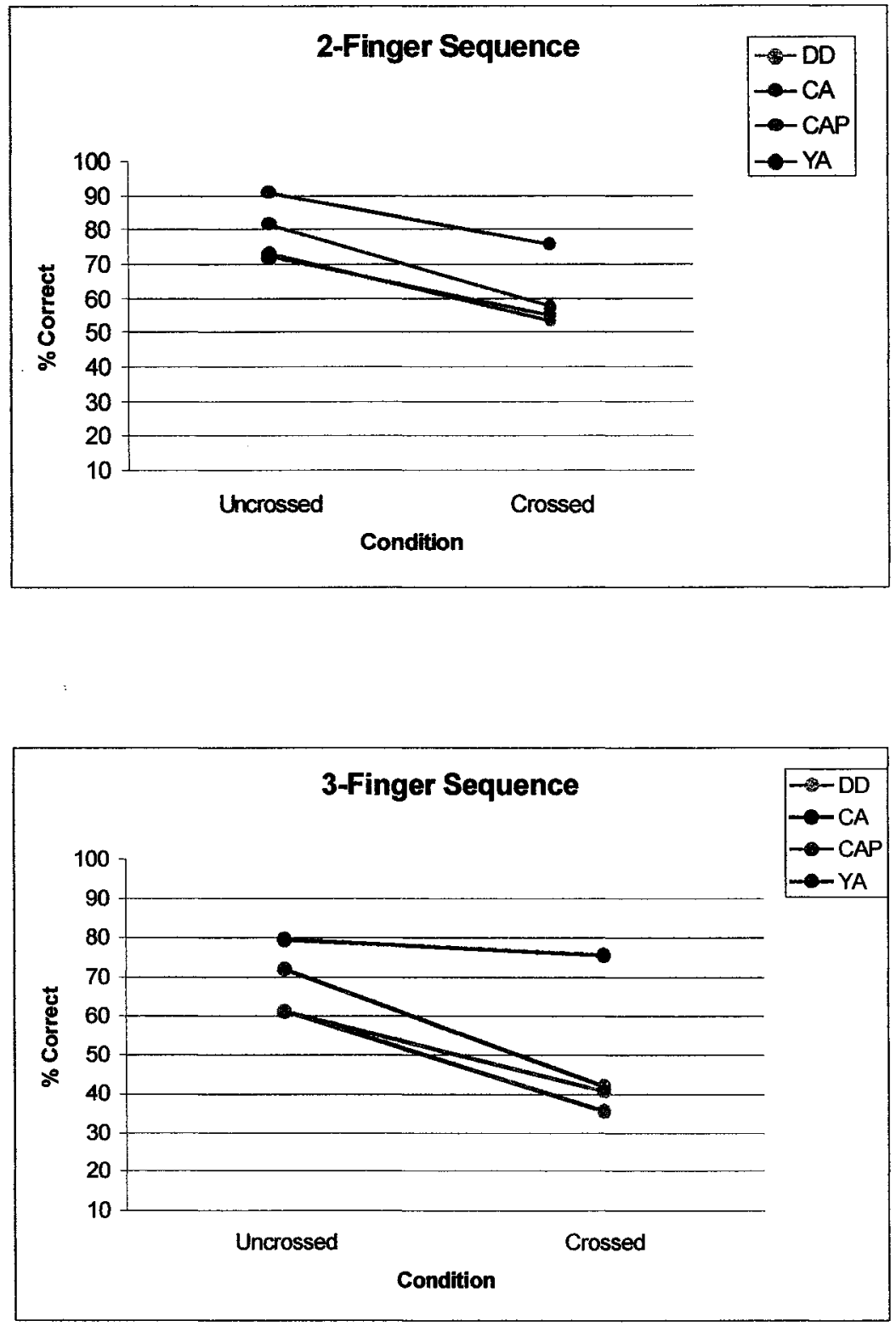


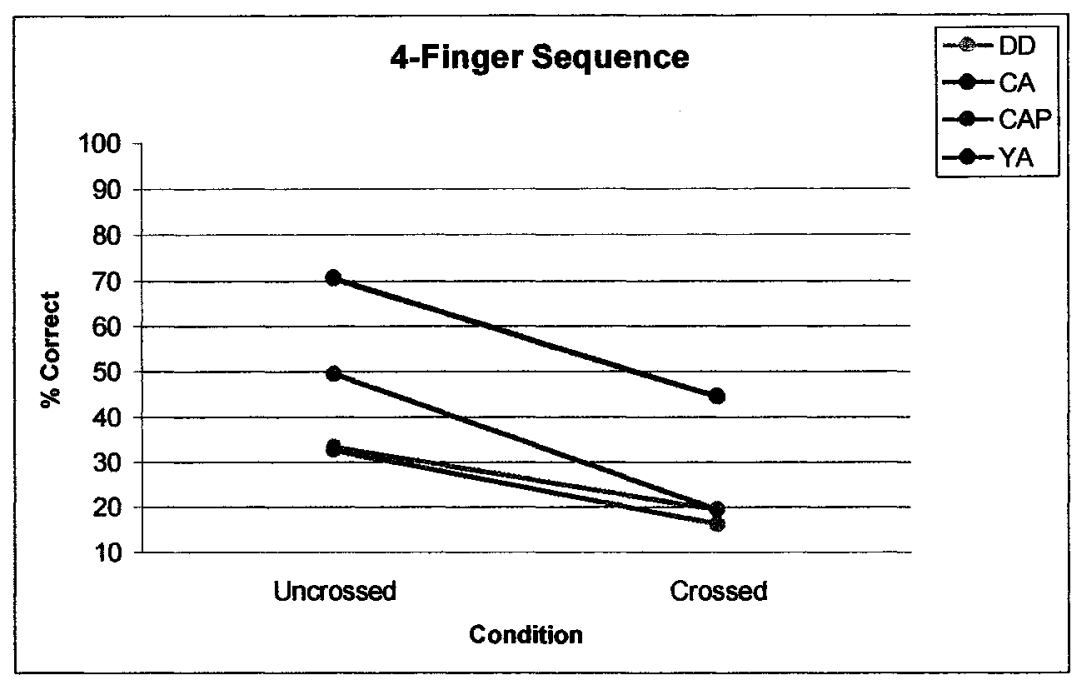

Figure 10.1. Mean accuracy and standard errors for 2-Finger, 3-Finger, and 4-Finger Sequences.

The CUD (Crossed-Uncrossed-Difference) was analyzed by ANOVA with mixed repeated-measures. There was no main and interaction effect of Hand, $F(1,67)=.977, p$ $=.326, \mathrm{y}^{2}=.014$. This indicated that this independent variable did not have any impact on the dependent variable. The data were then reanalyzed, collapsing across the left and right hands. There was an interaction of Group x Finger Sequence, $F(6,274)=2.10, p<$ $.05, \eta^{2}=.044$. Pairwise comparisons revealed that in the 4-Finger Sequence, the DD group $(M=13.95 \%, S D=15.73)$ demonstrated significantly less information degradation than the CA group $(M=26.20 \%, S D=22.0), p=.006$, and the CAP group $(M=30.00 \%$, $S D=19.98), p=.005$. The data are presented in Table 3 and summarized in Figure 10.2. 
Table 10.3

Mean Percentage of Crossed-Uncrossed Difference for Four Groups Crossed-Uncrossed-Difference (CUD)

\begin{tabular}{cccc}
\hline Group & $\begin{array}{c}\text { 2-Finger Sequence } \\
\text { Mean } \%(S D)\end{array}$ & $\begin{array}{c}\text { 3-Finger Sequence } \\
\text { Mean \% }(S D)\end{array}$ & $\begin{array}{c}\text { 4-Finger Sequence } \\
\text { Mean \% }(S D)\end{array}$ \\
\hline DD & $20.00(23.37)$ & $20.26(23.42)$ & $13.95(15.69)$ \\
CA & $15.20(18.21)$ & $21.20(20.96)$ & $26.20(23.11)$ \\
CAP & $24.00(13.92)$ & $30.00(17.77)$ & $30.00(20.00)$ \\
YA & $17.35(21.08)$ & $25.59(21.77)$ & $21.1320 .94)$ \\
\hline
\end{tabular}

Note: $\mathrm{DD}=$ Developmental dyslexic group; $\mathrm{CA}=$ Chronological age-matched group;

$\mathrm{CAP}=$ Chronological age-matched poor reader group; $\mathrm{YA}=$ Young age group.

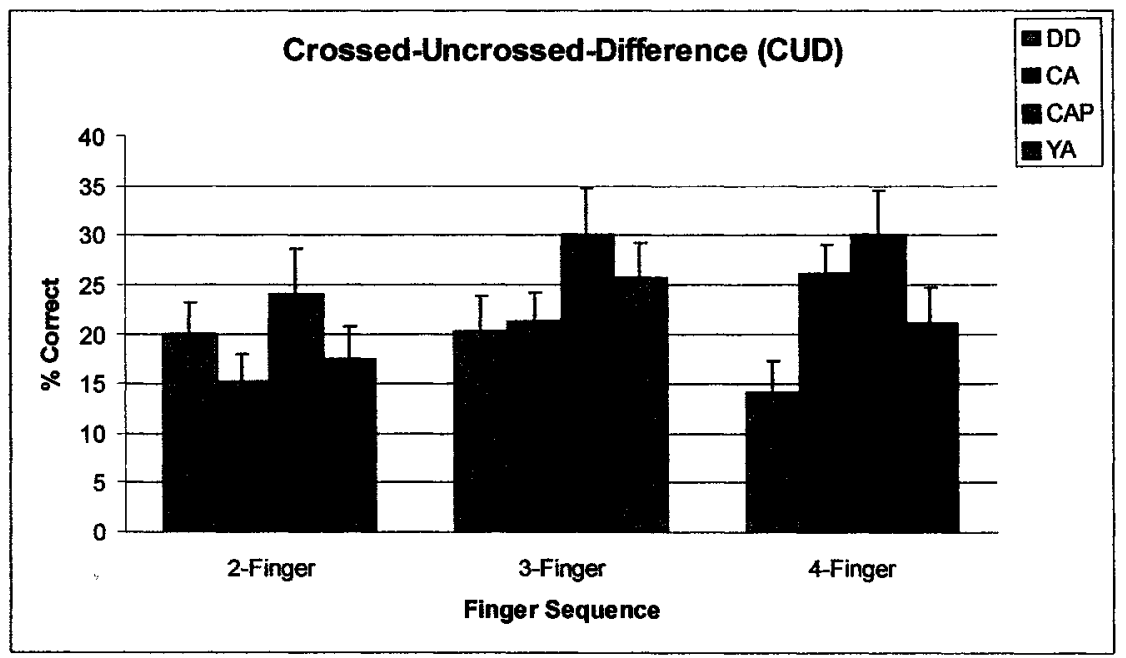

Figure 10.2. Mean accuracy and standard errors of Crossed and Uncrossed Difference.

The sequence errors were analyzed by an ANOVA with mixed repeated-

measures. There was no main and interaction effect of Hand, $F(1,67)=.209, p=.649$, $\mathrm{n}^{2}=.003$. This indicated that this independent variable did not have any impact on the dependent variable. The data were then reanalyzed, collapsing across the left and right hands. The analysis revealed that there were three main effects: Group, $F(3,138)=3.82$, $p<.011, \mathrm{y}^{2}=.077$, Condition, $F(1,138)=95.67, p<.000, \mathrm{y}^{2}=.409$, and Finger 
Sequence, $F(2,137)=65.85, p<.000, \mathrm{n}^{2}=.505$. There was also an interaction of Group $\mathrm{x}$ Finger Sequence, $F(6,274)=2.81, p<.011, \mathrm{y}^{2}=.058$.

Regarding Group, pairwise comparisons revealed that the CA group ( $M=8.77 \%$, $S D=9.64)$ demonstrated the least sequence errors followed by the YA group $(M=$ $9.80 \%, S D=9.42)$ and the $\mathrm{DD}$ group $(M=11.32 \%, S D=10.04)$. The CAP group $(M=$ $12.83 \%, S D=12.05$ ) made more sequence errors than the CA group, $p=.003$. Regarding Finger Sequence, more sequence errors were made in the 4-Finger Sequence $(M=$ $17.64 \%, S D=14.29)$ than the 2-Finger $(M=4.93 \%, S D=6.7)$ and 3-Finger Sequence $(M=9.47 \%, S D=9.83), p=.000$. A significant difference was also found between the 2Finger and the 3-Finger Sequences, $p=.000$. Regarding the interaction of Group $\mathrm{x}$ Finger Sequence, the DD group $(M=11.58 \%, S D=10.46)$ made significantly more sequence errors in the 3-Finger Sequence than the CA group $(M=3.8 \%, S D=8.55), p=$ .001 . The YA group $(M=11.58 \%, S D=9.37)$ also made significantly more sequence errors than the CA group, $p=.006$ in the 3-Finger Sequence. In the 4-Finger Sequence, the CAP group $(M=22.50 \%, S D=20.17)$ made significantly more sequence errors than the YA group $(M=13.97 \%, S D=12.94), p=.005$.

Finally, an analysis was conducted on the correlation between task performance and reading scores, nonverbal IQs, and age. As shown in Table 10.3, the Pearson product-moment correlation coefficient revealed a significant correlation between the reading scores and task performance scores in the Uncrossed and Crossed conditions. 
Table 10.4

Correlations of Finger Localization Task Scores, Age, Standardized TONI-3and TOWRE Scores

\begin{tabular}{llll}
\hline & Age & TONI-3 & TOWRE \\
\hline 2-finger sequence & & & \\
Uncrossed & .076 & .018 & $.272^{*}$ \\
$\quad$ Crossed & .126 & $.243^{*}$ & $.284^{*}$ \\
3-finger sequence & & & \\
$\quad$ Uncrossed & $.209^{*}$ & .172 & .197 \\
$\quad$ Crossed & $.204^{*}$ & .211 & $.277^{*}$ \\
4-finger sequence & & & \\
$\quad$ Uncrossed & $.391^{* *}$ & .017 & $.275^{*}$ \\
$\quad$ Crossed & $.301^{* *}$ & .135 & $.497^{* *}$ \\
\hline
\end{tabular}

Note: TONI-3 $=$ Test of Nonverbal Intelligence-3; TOWRE $=$ Test of Word Reading Efficiency.

2-tailed test. ${ }^{*} p<.05 . * * P<.01$.

\subsection{Discussion}

The principal aim of this experiment was to investigate interhemispheric communication associated with DD in tactile information transfer. The main significant findings were: (1) all groups demonstrated that finger localization was more accurate in the Uncrossed than the Crossed condition; (2) the CA group demonstrated the highest accuracy scores among the groups; (3) sequence errors increased with the number of finger sequences across the four groups, (4) the DD group made more sequence errors than the CA group; and (5) the reading scores and finger localization scores were correlated in both the Uncrossed and Crossed conditions.

As expected, all groups demonstrated lower accuracy in the Crossed than Uncrossed conditions (Prediction 1). This validates previous findings that callosal transfer causes degradation of information (Geffen et al., 1985). This finding is also consistent with Summerfield and Michie's 1993 study. As expected in Prediction 2, the CA group obtained higher task scores than the DD, CAP and YA groups in the Crossed condition (Fletcher et al., 1982; Lindgren, 1978; Moore et al., 1996; O'Donnell, 1983; 
Zung, 1986). This suggests that callosal information transfer is related to age and reading ability.

It was unexpected that the DD group ( $M$ reading age of 8.01 years) and YA group ( $M$ reading age of 12.06 years) did not significantly differ in the Crossed condition (Prediction 2). This finding did not accord with Summerfield and Michie's (1993) finding, i.e., their DD children ( $M$ age $=9.0$ years) had significantly lower task scores than the younger control group ( $M$ age $=7.0$ years) in the Crossed condition. Because the DD group demonstrated similar task scores to the YA group in the present experiment, it would appear that the developmental delay model could account for DD (Gough \& Turner, 1986). According to Frith's stage model (1985) of reading acquisition, children normally pass through the three developmental stages of reading acquisition, i.e., logographic, alphabetical and orthographic, sequentially and over a period of time, in order to master reading skills before they become fluent readers. Geffen et al. (1985) provided evidence that callosal efficiency normally improves with age and callosal maturity. Thus, the developmental delay model assumes that DD children may take more time to go through these developmental stages of reading acquisition and the developmental processes of callosal efficiency (i.e., myelination) than normal children do; however, the model also asserts that eventually DD children can catch up with normal children. That is, although callosal efficiency normally improves with age (Geffen et al., 1985), DD children may go through these developmental stages more slowly than normal children do. Nevertheless, this model cannot provide a full explanation as to why the DD group ( $M$ age $=13.04$ years, $M$ reading age $=8.02$ years), who were much older and less skilled in reading than the YA group $(M$ age $=9.01$ years, 
$M$ reading age $=12.06$ years), demonstrated similar patterns of performance to the YA group.

An alternative explanation is that the difference in educational training between the two DD groups could have been reflected in the negative result, since the $\mathrm{DD}$ children ( $M$ age of 13.08 year) in the present experiments were mainly drawn from the Provincial Demonstration Schools, where they had been exposed to excellent educational programs for years; (small classes and instructions geared toward their learning disabilities). Thus, their good training might have compensated for inefficient interhemispheric communication, and may have resulted in their catching up with the YA group. In contrast, the DD children in Summerfield and Michie's study were quite young, and might not have had enough training to compensate for inefficient interhemispheric communication, which might have resulted in their scores being lower than those of the younger control group.

Interestingly, the CAP group did not show a significant difference in performance from either the DD group or the YA group; however, this group had significantly lower scores than the CA group in the Crossed condition. This suggests that the Crossed finger localization task might be associated with poor reading ability in general.

It was unexpected that there was no Group $\mathrm{x}$ Condition interaction. All groups demonstrated a similar degree of degradation of callosal information transfer, confirmed by the absence of Group effect in the CUD scores (Prediction 3). Thus, these negative findings do not support the hypothesis of the present thesis that DD is associated with deficits in interhemispheric communication. The data also did not support Prediction 4 
that callosal efficiency of tactile information transfer is correlated with age and developing maturity of the $\mathrm{CC}$.

However, the unexpected significant difference between the DD and CA groups in the 2-finger sequence in the Uncrossed condition might have contributed to these negative findings. It was predicted that all four groups would show similar scores in the 2-finger sequence in the Uncrossed condition because this was an easy task in terms of little memory involvement and no requirement for callosal information transfer (Prediction 5). Thus, it was surprising that the DD and YA groups demonstrated significantly lower scores than the CA group in this Condition. Although this finding was consistent with Summerfield and Michie's study (1993), their DD children ( $M$ age of 9.0 years) were much younger than the DD students ( $M$ age of 13.08 years) in the present study. Summerfield and Michie commented that the $\mathrm{CC}$ might have a role in sequential processing of intrahemispheric information processing because callosal agenesis patients (Lassonde et al., 1988) and commissurotomized patients (Geffen et al., 1985) showed lower scores than their control groups in the 2-finger sequence but not in the single finger localization in the Uncrossed condition. Moore et al. (1996) also reported a similar negative finding with adult dyslexics in a 3-finger localization task. They found a highly significant difference in both the Uncrossed and the Crossed conditions but not in the CUD in comparing 21 right-handed DD adults $(M$ age $=24.76$ years $)$ and 21 righthanded university students ( $M$ age $=23.81$ years). Because Summerfield and Michie's interpretation is still speculative, it is unclear why the DD and CA group significantly differ in the 2-Finger Sequence in the Uncrossed condition. As Summerfield and Michie 
suggested, further research is necessary to investigate the possibility of callosal roles in intrahemispheric information processing.

With respect to the CUD, it was unexpected that the DD group did not demonstrate degradation of information transfer compared to the other groups. A possible explanation is that the fact that the DD group performed significantly more poorly than the CA group in the Uncrossed condition which might create a floor effect, since the CUD is assumed to reflect additional degradation of information transfer via the CC. A typical example of this pattern was observed in the 4-Finger Sequence; the DD group had a smaller CUD than the CA and CAP groups, perhaps because the DD group responded less accurately in the Uncrossed condition.

An alternative explanation is that the small effect size $\left(\eta^{2}=.046\right)$ of main effect of Group in the CUD data might have an effect on statistical power, defined as the probability of rejecting the null hypothesis when it is actually false (Howell, 1987). Cohen (1977) characterized eta squared $\left(\mathrm{\eta}^{2}\right)$ as follows: $\eta^{2}=.01$ as small, $\mathrm{\eta}^{2}=.06$ as medium, and $\mathrm{y}^{2}=.14$ as a large effect size when total sample size is about 50 or more. Since the total sample size in the present study was 71 , the observed effect size of .046 is relatively 'small' based on Cohen's characterization. Third, because students in the DD group were mostly drawn from the Ontario Demonstration Schools, these participants had been exposed to excellent educational programs for years, which might have helped them develop efficient interhemispheric communication strategies.

As expected, the DD group made more sequence errors than the CA group in the 3-Finger Sequence (Prediction 6). Interestingly, the DD group, however, did not differ from the CAP and YA groups in the sequence errors across the Finger Sequences. 
As expected, standardized reading scores of the TOWRE were positively correlated with the 2-Finger, 3-Finger and 4-Finger Sequence scores in the Crossed condition. However, the 2-Finger and 4-Finger Sequence scores also correlated with the TOWRE scores in the Uncrossed condition. This suggests that reading ability is associated with intra- and interhemispheric communication. This finding supports the hypothesis that interhemispheric communication is associated with reading ability (Prediction 7).

Finally, the DD group performed similarly to the CAP group across the tasks and conditions. These similar patterns of performance do not support the hypothesis that DD is qualitatively distinct from general poor reading ability (Prediction 8). Instead, the similarities suggest that inefficient interhemispheric communication is associated with poor reading in general, including among developmental dyslexics.

Experiment 1 demonstrated findings similar to Summerfield and Michie's (1993) in terms of the following points: (1) both the DD and YA groups had lower scores than the CA group in both the Crossed and Uncrossed conditions; and (2) there were significant correlations between the standardized reading scores and finger localization task scores in the Crossed condition. However, the present study differed from Summerfield and Michie's (1993) in terms of the following points: (1) there was no significant difference between the DD and YA groups across the conditions, and (2) there was a significant correlation between the standardized reading scores and finger localization task scores in the Uncrossed condition. The first discrepancy between the two studies might be explained by compensation due to excellent training programs. The second might be explained by the different standardized reading tests (TOWRE vs. 
Schonell Graded Word Reading Test) used in the two studies, i.e., the two standardized reading tests might assess different reading components. Nevertheless, the similar findings to Summerfield and Michie's study have achieved the main goal of this experiment: establishing the foundation of the present thesis in terms of the involvement of inefficient interhemispheric communication in DD.

The overall similarities between the DD and CAP group suggest that inefficient interhemispheric communication is not specific to the DD group. This implication does not support the hypothesis that DD is a distinct reading disability. More importantly, however, the findings do suggest the possibility that poor somato-sensory movements are common in both DD and CAP groups. Since Summerfield and Michie (1993) did not include a CAP group in their study, this possibility was not explored. More studies are needed to investigate this possibility.

In conclusion, the similarity of findings between Experiment 1 and Summerfield and Michie's study established the foundation for the present thesis: that there are associations between DD and inefficient interhemispheric communication. However, the findings also imply the possibility of inefficient interhemispheric communication that might be a common characteristic of DD and poor reading ability. Further research is needed to investigate this possibility. 


\section{Chapter 11: Experiment 2}

The aim of Experiment 2 was to investigate inefficient interhemispheric communication associated with DD. A rhyming judgment task was used in a visual-half field paradigm to examine phonological information processing associated with interhemispheric communication involving the genu (corresponding to the $\mathrm{CC} \# 2$ in Figure 5.2). Although the detailed rationale for Experiment 2 was discussed in Chapter 8.3.2, three points should be highlighted in order to formulate specific predictions for the interhemispheric inefficiency hypothesis and the specific reading disorder hypothesis. First, the LH is primarily specialized for phonological processing (e.g., Gross et al., 1978; Krutsch \& McKeever, 1990; Ortells et al., 1998; Yeni-Komshian et al., 1975). Second, phonological skills are a good predictor for reading ability (Adams, 1990; Baron, 1979; Doctor \& Coltheart, 1980; Ehri \& Wilce, 1979; Stanovich, 1982). Third, interhemispheric communication enhances efficiency in information processing for complex tasks (cf. Banich \& Belger, 1990; Belger \& Banich, 1992). Based on these points, the following predictions were formulated with regard to the expected performance of the four groups (DD, CA, CAP, YA) in the rhyming judgment task: Prediction 1: The RVF superiority effect would be observed in all four groups because the LH is primarily specialized for processing linguistic stimuli.

Prediction 2: The CA ( $M$ age $=13.04$ years $)$ group would respond the fastest and most accurately among the groups, the CAP $(M$ age $=13.09$ years $)$ and YA $(M$ age $=9.01$ years) groups would demonstrate similar performance patterns, 
and the DD ( $M$ age $=13.08$ years) group would respond the slowest and least accurately among the groups due to age and reading ability.

Prediction 3: The CA and CAP groups would respond faster and more accurately in the Bilateral RVF Condition than in the Unilateral RVF Condition due to a combination of efficient information processing from the bilateral presentations and linguistic specialization of the LH.

Prediction 4: The YA group would respond more slowly and less accurately in the Bilateral RVF Condition than in the Unilateral RVF Condition due to inefficient information processing from the bilateral presentation.

Prediction 5: The DD group would respond more slowly and less accurately in the Bilateral RVF Condition than in the Unilateral RVF Condition due to a combination of inefficient information processing from the bilateral presentations.

Prediction 6: Standardized reading (TOWRE) scores would correlate with RT and accuracy in the Bilateral RVF Condition due to the linguistic specialization of the LH.

Prediction 7: If DD is qualitatively distinct from poor reading ability, the DD group would show different patterns of performance from the CAP group. 


\subsection{Method}

\subsubsection{Apparatus}

All visual stimuli were presented on a $15 "$ CRT monitor. Stimulus events and timing operations were controlled by an IBM ThinkPad LCD computer system. E-Prime 1.0 (Psychology Software Tools Inc., 2000), a PC computer software program designed for Windows, was used to present the stimuli. The viewing distance was held constant at $57 \mathrm{~cm}$ (E-Prime User's Guide, 2001). A standard chin and headrest (Table model of Veatch Ophthalmic Instruments) was used to fix the distance.

\subsubsection{Stimulus Materials}

The four stimulus lists used in the experiment are reproduced in Appendix N. Two types of stimuli pairs were used: rhymed or non-rhymed. One half of these pairs were orthographically similar rhymed (e.g., beach-peach) or non-rhymed (e.g., hell-hall). The other half of the pairs were orthographically dissimilar rhymed (e.g., whale-scale) and non-rhymed (chat-gate). The total number of word pairs was 30 (Appendix N). All words consisted of from three to six letters with no more than two syllables. The words were chosen from the Penguin Rhyming Dictionary (Fergusson, 1983). Word length and frequency in relation to the second word of the pairs were controlled across the four lists (Francis \& Kućera, 1982).

\subsubsection{Procedure}

Participants were individually tested in a small quiet room in their schools or in a laboratory setting at Carleton University. The participant was seated on a chair in front of 
a computer monitor. The procedure was illustrated on an information sheet with examples of stimuli (two examples of rhymed and non-rhymed pairs). While the participant viewed the illustration, the procedure and the examples were explained. The participant was instructed to place his or her chin on a chin-rest, his or her forehead against the headrest, and to look straight ahead at a dot on the screen throughout each trial. The participant was encouraged to guess if he or she was not sure of the answer. All stimuli were presented in Courier New 13 font size in black print on a white screen at $2.5^{\circ}$ horizontally to the left or right of the center fixation dot (font size 40 ) on the monitor. Thus, even if small eye movements occurred, projection of the image to the opposite hemisphere was ensured (Banich, 1997). In every trial, the total word length was located between $2.5^{\circ}$ and $1.5^{\circ}$ from the center fixation dot (Brysbaert \& $D^{\prime} Y$ dewalle, 1990; Fuentes \& Tudela, 1992).

As shown in Figure 11.1, at the beginning of the trial, a fixation dot flashed twice in the center of the monitor as a starting signal. The third fixation dot then appeared and stayed in the center throughout the trial. The visual half-field presentation procedure (see Appendix A) requires participants to focus on the center during the trial, and thus, the fixation dot was necessary to minimize eye movements (Banich, 1997). 500-msec after the third fixation dot appeared on the screen, the first word was presented in either the RVF/LH or the LVF/RH for 150 msec. Sequentially, the second word was presented in the same VF (Unilateral Condition) for $150 \mathrm{msec}$. This duration for the words selected was used in a previous study (Brysbaert \& D'Ydewalle, 1990). For the Bilateral Condition, the first word was presented in either the RVF/LH or the LVF/RH, and sequentially, the second word was presented on the opposite VF to the initial 
presentation. The interval between the first and second words (interstimulus interval, ISI) was $500 \mathrm{msec}$.

Reaction time (RT) and accuracy were recorded by the computer. Four Conditions (i.e., Unilateral LVF, Bilateral LVF, Unilateral RVF, and Bilateral RVF) were completed by each participant. At the beginning of each Condition blocked by trials, the participant was instructed on which index finger was to be used to click the mouse for response. When the right index finger was used, 30 pairs of stimuli were presented in the RVF as test trials and 6 pairs of stimuli were presented in the LVF. The latter pairs were fillers, which were not included in the analysis. This manipulation was necessary in order to prevent the participant's expectation that a stimulus was coming from one VF. When the left index finger was used, the condition was reversed. The participant was instructed to respond to the second word by clicking a mouse with the instructed index finger, labeled 'Yes' (right side of the mouse) for a rhymed pair and 'No' (left side of the mouse) for a non-rhymed pair, as quickly and accurately as possible. There was no time limit for the response; however, if the participant did not respond after more than five seconds, he or she was encouraged to respond by the experimenter. A total of 12 practice trials was provided, followed by 36 trials ( 30 test trials and 6 fillers) with auditory feedback (i.e., happy sound) for correct responses. The four stimulus lists were used for the Unilateral and Bilateral Conditions. Thus, eight combinations (4 stimulus lists $\times 2$ conditions) were counterbalanced in each participant. The order of the stimuli in each list was randomized by the computer for each participant.

To establish the test-retest reliability of the visual half-field presentation procedure for the present experiment, one 12 year-old girl (an acquaintance of the author) 
was tested twice within 10 days to examine whether the results of the two tests were consistent. An ANOVA revealed that a Group x Test interaction was not significant, $F$ $(1,46)=2.37, p=.131, \mathrm{n}^{2}=.049$. This result ensured that the test-retest reliability of the present visual half-field presentation procedure was adequate. The data are summarized in Appendix $\mathrm{O}$.

\section{A rhyming judgment task}

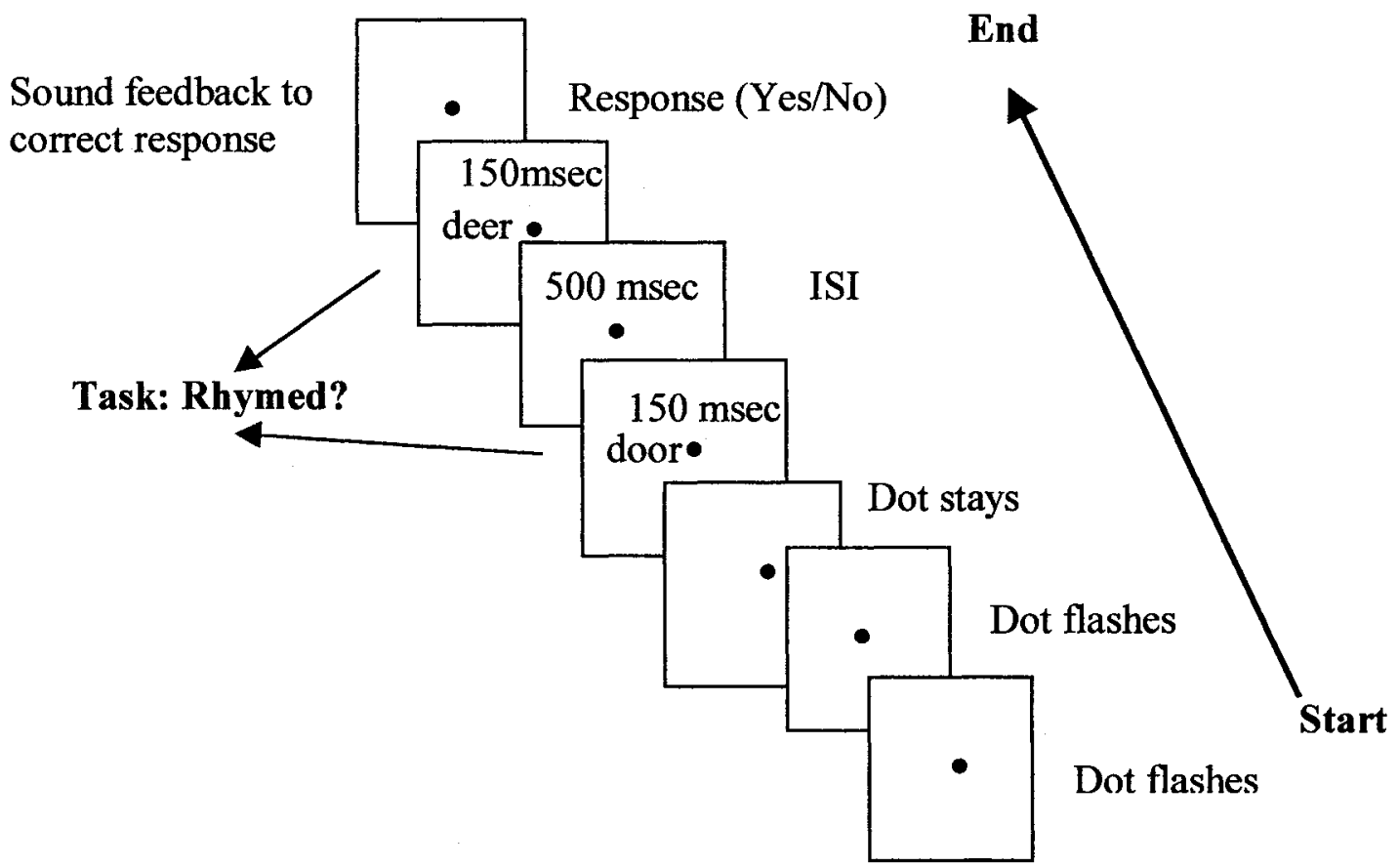

Figure 11.1. Procedure for the rhyming judgment task.

The dot flashes twice, then stays in the center of the screen. The first word appears for $150 \mathrm{msec}$ in either the RVF or LVF followed by a $500 \mathrm{msec}$ ISI (interstimulus interval). The second word then appears for $150 \mathrm{msec}$ in the same visual field for the Unilateral Condition and the other visual field for the Bilateral Condition. The participant makes a judgment as to whether the words are rhymed as quickly and accurately as possible. 


\subsubsection{Analyses}

A 4 (Group: CA, CAP, DD, YA) x 2 (Visual Field: right, left) x 2 (Condition: bilateral, unilateral) MANOVA with mixed repeated-measures design was used. Group was a between-groups factor, and Visual Field and Condition were within-group factors. The dependent variables (DVs) were the latency to correct responses and the percentage of correct responses. Significant effects were subsequently investigated using the Bonferroni corrected $\alpha$ for pairwise comparisons. As Experiment 1, the Bonferroni test divided $\alpha$ level (.05) by the number of comparisons to control the chance of Type I errors (Stevens, 1996).

\subsection{Results}

As in Experiment 1, preliminary analyses were conducted to screen the data in terms of: (1) the presence of ceiling and floor effects, (2) the presence of extreme values (i.e., values exceeding 3 standard deviations, $S D$ ) in each group, (3) the observations on the dependent variables (DVs) being normally distributed in each group, and (4) the observations on the DVs having equal variances for the groups. The ceiling and floor effects indicate that the observations are not distributed as expected. In terms of the Box plot test, the Box had a reasonable size and the fact that the line representing the median inside the Box did not reach either edge of the Box in all observations suggests that there was no ceiling and floor effect in the data. The Box plot test also identified extreme values in the data. The Shapiro-Wilk test for normality and the Levene test for homogeneity of variance indicated the MANOVA assumptions were not met. The violation of these assumptions has a substantial effect on power. Thus, to solve the 
problems of extreme values and violations of the assumption, it was decided to use the medians instead of the means from individual scores to calculate group means (Stevens, 1996). The group means of the median RTs and the percentage of correct responses are presented in Table 11.1.

In another preliminary analysis, a MANOVA was performed to examine the possible between-group and within-group differences on Gender and Handedness. There were no significant main effects and interactions with Visual Field, Condition and Group. Therefore, these data were pooled across Gender and Handedness variables.

Table 11.1

Group Means of Reaction Times (msec) and Percentage of Correct Responses for Each of Four Groups

\begin{tabular}{|c|c|c|c|c|c|c|c|c|}
\hline \multirow[b]{3}{*}{ Group } & \multicolumn{4}{|c|}{$\begin{array}{l}\text { Reaction Time (msec) } \\
\text { Conditions }\end{array}$} & \multicolumn{4}{|c|}{$\begin{array}{c}\text { Accuracy }(\%) \\
\text { Conditions }\end{array}$} \\
\hline & \multicolumn{2}{|c|}{$\begin{array}{l}\mathrm{LVF}(M) \\
(S D)\end{array}$} & \multicolumn{2}{|c|}{$\begin{array}{c}\operatorname{RVF}(M) \\
(S D) \\
\end{array}$} & \multicolumn{2}{|c|}{$\begin{array}{c}\operatorname{LVF}(M) \\
(S D)\end{array}$} & \multicolumn{2}{|c|}{$\begin{array}{c}\mathrm{RVF}(M) \\
(S D)\end{array}$} \\
\hline & Unilateral & Bilateral & Unilateral & Bilateral & Unilateral & Bilateral & Unilateral & Bilateral \\
\hline DD & $\begin{array}{r}1153.76 \\
(338.81)\end{array}$ & $\begin{array}{r}1172.79 \\
(342.72)\end{array}$ & $\begin{array}{r}1127.37 \\
(300.33)\end{array}$ & $\begin{array}{r}1189.92 \\
(289.36)\end{array}$ & $\begin{array}{r}59.82 \\
(11.47)\end{array}$ & $\begin{array}{r}62.45 \\
(10.17)\end{array}$ & $\begin{array}{r}63.86 \\
(14.61)\end{array}$ & $\begin{array}{r}62.11 \\
(10.08)\end{array}$ \\
\hline $\mathrm{CA}$ & $\begin{array}{r}924.60 \\
(203.20)\end{array}$ & $\begin{array}{r}918.06 \\
(291.88)\end{array}$ & $\begin{array}{r}865.06 \\
(183.60)\end{array}$ & $\begin{array}{r}862.60 \\
(181.87)\end{array}$ & $\begin{array}{r}70.48 \\
(12.52)\end{array}$ & $\begin{array}{r}72.64 \\
(12.53)\end{array}$ & $\begin{array}{r}74.04 \\
(14.61)\end{array}$ & $\begin{array}{r}69.32 \\
(14.09)\end{array}$ \\
\hline CAP & $\begin{array}{r}989.80 \\
(483.84)\end{array}$ & $\begin{array}{r}1042.15 \\
(428.25)\end{array}$ & $\begin{array}{r}1058.25 \\
(469.19)\end{array}$ & $\begin{array}{r}911.80 \\
(444.00)\end{array}$ & $\begin{array}{r}60.20 \\
(11.91)\end{array}$ & $\begin{array}{r}66.20 \\
(11.39)\end{array}$ & $\begin{array}{r}68.80 \\
(11.55)\end{array}$ & $\begin{array}{r}62.30 \\
(14.42)\end{array}$ \\
\hline YA & $\begin{array}{r}1220.65 \\
(344.16) \\
\end{array}$ & $\begin{array}{r}1201.91 \\
(421.94)\end{array}$ & $\begin{array}{r}1111.94 \\
(340.79)\end{array}$ & $\begin{array}{r}1122.35 \\
(332.94)\end{array}$ & $\begin{array}{r}62.75 \\
(16.25)\end{array}$ & $\begin{array}{r}69.41 \\
(14.64)\end{array}$ & $\begin{array}{r}72.95 \\
(15.85)\end{array}$ & $\begin{array}{r}63.33 \\
(14.68)\end{array}$ \\
\hline
\end{tabular}

Note: $\mathrm{LVF}=$ left visual field; $\mathrm{RVF}=$ right visual field; $M=$ mean; $S D=$ standard deviation; $\mathrm{DD}=$

Dyslexic group; $\mathrm{CA}=$ Chronological age-matched group; $\mathrm{CAP}=$ Chronological age-matched poor reader group; YA: Young group.

For the main analysis, a 4 (Group: DD, CA, CAP, YA) x 2 (Condition: bilateral, unilateral) $\times 2$ (Visual Field: right, left) MANOVA with mixed repeated measures was performed on the group means of the RT and the percentage of correct response data. The analysis revealed a three-way interaction of Condition $\mathrm{x}$ Visual Field $\mathrm{x}$ Group, $F(6$, 
$132)=2.7, p<.05, \mathrm{\eta}^{2}=.104$, a two-way interaction of Condition $\mathrm{x}$ Visual Field, $F(2$, $66)=14.2, p<.000, \mathrm{y}^{2}=.301$, and two significant main effects: Group, $F(6,132)=$ $4.41, p<.000, \mathrm{y}^{2}=.167$, and Visual Field, $F(2,66)=3.79, p<.05, \mathrm{y}^{2}=.004$. The data are summarized in Figure 11.2 (RT) and Figure 11.3 (accuracy).

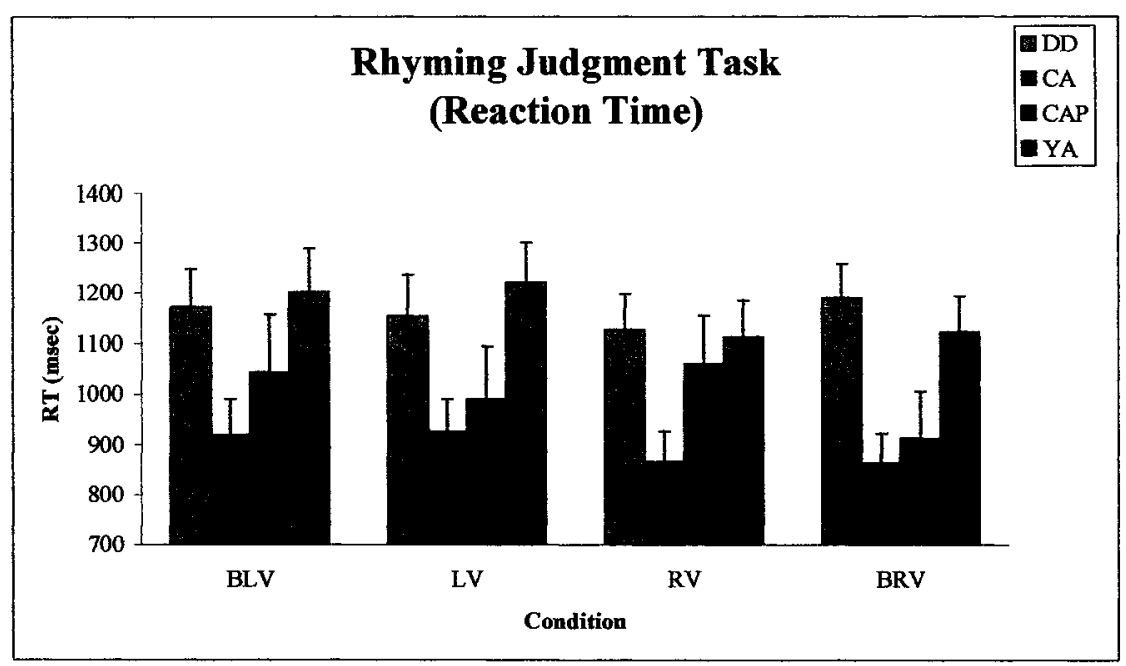

Figure 11.2. The interaction of Group $x$ Visual Field $x$ Condition in reaction time.

There is no significant difference between the Unilateral and Bilateral Conditions across groups in the left visual field. DD = Dyslexic group $(n=19)$; $C A=$ Chronologically agematched group $(n=25)$; CAP = Chronologically age-matched poor reader group $(n=10)$; $Y A=$ Young age group $(n=17) ; L V=$ Unilateral left visual field; $B L V=$ Bilateral left visual field; $\mathrm{RV}=$ Unilateral right visual field; $\mathrm{BRV}=$ Bilateral right visual field. The arrows indicate the standard error. ${ }^{*} p<.003$. 


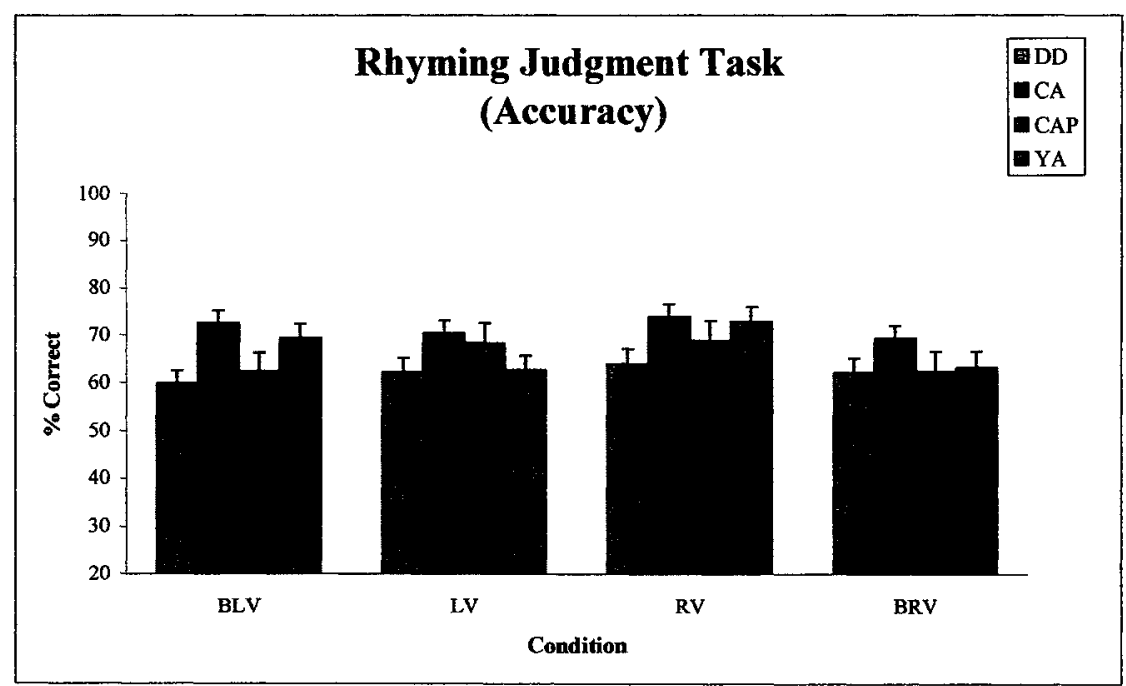

Figure 11.3. The interaction of Group $x$ Visual Field $x$ Condition in accuracy.

There was no significant difference among groups across the Conditions.

The arrows indicate standard error. ${ }^{*} p<.001$.

Pairwise comparisons revealed that the DD group $(M=1189.92 \mathrm{msec})$ responded significantly more slowly than the CA group $(M=862.60 \mathrm{msec})$ in the Bilateral RVF Condition, $p=.001$. The difference between the YA $(M=1122.35 \mathrm{msec})$ and CA groups approached the significant level in the Bilateral RVF Condition, $p=.007$. The DD group performed similarly to the CAP $(M=911.80 \mathrm{msec})$ and YA groups in the Bilateral RVF Condition. The RT scores of the CAP group also differed significantly from the CA group. The difference between the DD $(M=1127.37 \mathrm{msec})$ and CA $(M=865.06 \mathrm{msec})$ groups approached the significant level in the Unilateral RVF Condition, $p=.006$. The difference between the YA $(M=1220.65)$ and CA $(M=924.60 \mathrm{msec})$ groups also approached the significant level in the Unilateral LVF Condition, $p=.005$. The accuracy data, however, revealed no significant group difference. 
Pairwise comparisons revealed two significant findings of within Group factors. The CAP group responded significantly faster in the Bilateral RVF Condition $(M=$ $911.80 \mathrm{msec})$ than the Unilateral RVF Condition $(M=1058.25 \mathrm{msec}), p=.003$, whereas the accuracy did not significantly differ between the two Conditions. The YA group responded less accurately in the Bilateral RVF Condition $(M=63.33 \%)$ than in the Unilateral RVF Condition $(M=72.95 \%), p=.001$, whereas the RT did not significantly differ between the two Conditions. This indicates there was no speed-accuracy trade-off in these effects.

To further investigate the group differences in the Bilateral RVF Condition, the difference between the Bilateral and Unilateral Conditions was calculated. Another 4 (Group: CA, CAP, DD, YA) x 2 (Visual Field: left, right) MANOVA with mixed repeated-measures was conducted on the data. There was a significant Group x Visual Field interaction, $F(6,132)=2.55, p=.022, \eta^{2}=.104$, and a main effect of Visual Field, $F(2,66)=14.07, p=.000, \eta^{2}=.299$. Pairwise comparisons revealed that the RT difference between the DD group $(M \Delta=+62.56 \mathrm{msec})$ and CAP groups $(M \Delta=-$ $146.45 \mathrm{msec}$ ) was significant, $p=.001$. The accuracy was not significantly different across all four groups.

The main effect of Visual Field indicated that the words presented in the RVF/LH $(M=1031.16 \mathrm{msec}, S D=317.76 ; M=67.09 \%, S D=13.74)$ were responded to faster and more accurately than those presented in the LVF/RH $(M=1077.97 \mathrm{msec}, S D=$ $356.85 ; M=65.49 \%, S D=12.61)$. The absence of an interaction between Visual Field and Group confirmed the presence of the expected RVF superiority effect across the groups. The main effect of Group reflected the difference between the CA group ( $M=$ 
$892.58 \mathrm{msec})$ and the DD group ( $M=1160.96 \mathrm{msec}), p=.004$, and between the CA group and YA group $(M=1164.21 \mathrm{msec}), p=.005$. The DD, YA and CAP groups $(M=$ $1000.50 \mathrm{msec}$ ) did not significantly differ from each other, and the CA and CAP groups did not significantly differ from each other. In terms of accuracy, a significant difference was found only between the DD group $(M=62.06 \%)$ and the CA group $(M=69.32 \%)$.

Finally, a correlation analysis was performed to investigate the relationship between standardized reading scores of the TOWRE and accuracy and RT scores in the Bilateral RVF Condition. As shown in Table 11.2, the TOWRE scores were significantly correlated with RT and accuracy across the conditions. The PDE scores were negatively correlated with RT data in both the Bilateral RVF Condition $(r=-.265, p<.05)$ and Unilateral RVF Condition $(r=-.247, p<.05)$. The PDE scores were also correlated with accuracy data in the Unilateral RVF Condition $(r=.336, p<.01)$. In addition, the PDE scores were positively correlated with the accuracy data in both Bilateral LVF Condition $(r=.289, p<.05)$ and Unilateral LVF Condition $(r=.301, p<.05)$. The SWE scores were also positively correlated with the accuracy data in the Unilateral RVF Condition ( $r$ $=.336, p<.01)$, and in both Bilateral LVF Condition $(r=.342, p<.01)$ and Unilateral LVF Condition $(r=.288, p<.01)$. Age was negatively correlated with RT in both Bilateral LVF Condition $(r=-.285, p<.05)$ and Unilateral LVF Condition $(r=-.255, p<$ $.05)$. 
Table 11.2

Correlations of Phonological Task Scores with Age, TONI-3, and Sub-tests of TOWRE Scores

\begin{tabular}{|c|c|c|c|c|}
\hline Condition & Age & TONI-3 & SWE & PDE \\
\hline RT & & & & \\
\hline $\begin{array}{l}\text { Bilateral } \\
\text { RVF }\end{array}$ & -.230 & -.014 & -.160 & $-.265^{*}$ \\
\hline $\begin{array}{l}\text { Unilateral } \\
\mathrm{RVF}\end{array}$ & .221 & -.069 & -.150 & $-.247^{*}$ \\
\hline $\begin{array}{l}\text { Bilateral } \\
\text { LVF }\end{array}$ & $-.285^{*}$ & -.070 & -.119 & -.198 \\
\hline $\begin{array}{l}\text { Unilateral } \\
\text { LVF }\end{array}$ & $-.255^{*}$ & -.060 & -.098 & -.150 \\
\hline $\begin{array}{l}\text { Accuracy } \\
\text { Bilateral } \\
\text { RVF }\end{array}$ & .144 & .065 & & .168 \\
\hline $\begin{array}{l}\text { Unilateral } \\
\text { RVF }\end{array}$ & .111 & -.003 & $.336^{* *}$ & $.298^{*}$ \\
\hline $\begin{array}{l}\text { Bilateral } \\
\text { LVF }\end{array}$ & .032 & .024 & $.342^{* *}$ & $.289 *$ \\
\hline $\begin{array}{l}\text { Unilateral } \\
\text { LVF }\end{array}$ & .032 & .085 & $.288^{*}$ & $.301^{*}$ \\
\hline
\end{tabular}

Note: RT $=$ Reaction time; TONI-3 $=$ The of Nonverbal Intelligence- $3 ;$ Bilateral RVF $=$ Bilateral right visual field; Unilateral $\mathrm{RVF}=$ Unilateral right visual field; $\mathrm{SWE}=$ Sight Word Efficiency (subtest of the Test of Word Reading Efficiency); PDE = Phonological Decoding Efficiency (subtest of TOWRE). ${ }^{*} p<.05 ; * * p<.01$.

\subsection{Discussion}

The principal aim of this experiment was to investigate possible inefficient interhemispheric communication for phonological processing in DD children. The main findings are: (1) the RVF superiority was observed in all four groups; (2) the DD group responded significantly more slowly and less accurately than the CA group in the Bilateral RVF Conditions; (3) the YA group demonstrated significantly slower and fewer correct responses than the CA group in the Bilateral RVF Conditions; (4) the CAP group demonstrated faster responses in the Bilateral RVF than the Unilateral RVF Condition; (5) the YA group demonstrated fewer accurate responses in the Bilateral RVF than the 
Unilateral RVF Condition; (6) the TOWRE scores correlated with the RT and accuracy scores of the task in both the Bilateral and Unilateral Conditions.

As expected, the RVF superiority effect was observed across all groups, supporting Prediction 1. Target words presented in the RVF/LH were responded to faster and more accurately than those presented in the LVF/RH. This supports previous research indicating that the $\mathrm{LH}$ is primarily specialized for phonological processing (Sperry, 1974; Sperry et al., 1969; Zaidel, 1983). The absence of Group x Visual Field interaction confirms that all four groups demonstrated the RVF superiority effect. Since the RVF superiority effect is a typical finding in the visual half-field presentation when linguistic stimuli are used (Clarke \& Zaidel, 1994; Gross et al., 1978; Krutsch \& McKeever, 1990; Ortells et al., 1998; Yeni-Komshian et al., 1975; Clarke \& Zaidel, 1994), the presence of the RVF superiority effect validated the present visual half-field procedure. Furthermore, the presence of the RVF superiority effect in the DD group suggests that dysfunction in the LH cannot be a primarily factor in DD as the left hemisphere dysfunction theory proposed (e.g., Galaburda et al., 1985). As expected, the DD group responded significantly more slowly and less accurately than the CA group. The YA group responded less accurately but not more slowly than the CA group. Unexpectedly, the DD, YA, and CAP groups did not differ from each other, and the CAP group did not differ significantly from the CA group (as had been expected in Prediction 2). The lack of statistical differences among the DD, YA and CAP groups as well as between the CA and CAP groups indicates that this phonological task was associated with both reading ability and age related components. Since a close link between phonological skills and reading has been reported by other studies (e.g., Adams, 1990; 
Baron, 1979; Doctor \& Coltheart, 1980; Ehri \& Wilce, 1979; Stanovich, 1982), the present findings are consistent with these studies.

The observed multivariate three-way interaction of Group x Condition x Visual Field was particularly important because it demonstrated both efficiency (i.e., fast and accurate responses) and inefficiency (slow and inaccurate responses) of interhemispheric communication. One group, the CAP group, was significantly faster in the Bilateral RVF (146.45 $\mathrm{msec}$ ) than in the Unilateral RVF Condition (as expected in Prediction 3). Since accuracy was constant across the conditions, there was no speed-accuracy trade-off in this finding. This supports the view that interhemispheric communication was efficient for complex tasks (Banich \& Belger, 1992; Belger \& Banich, 1990). The CA group, however, did not show efficient information processing in bilateral presentation. A question arises as to why the CA group did not demonstrate the additional efficiency in interhemispheric communication, since they were good readers (mean reading age of 16.01 years). A possible explanation is that because the students in the CA group had quite a high reading age for their chronological age, their task performance might have been maximized, i.e., creating a ceiling effect, and might not have derived any evidence of efficiency from bilateral presentation.

Furthermore, the three-way interaction revealed that the YA group performed significantly less accurately (9.6\%) in the Bilateral RVF than in the Unilateral RVF Condition. Since the YA group did not show significant difference between the Bilateral RVF and Unilateral RVF Conditions in RT (10.5 msec), it is unlikely that a speedaccuracy trade-off resulted in this significant difference. Thus, in the younger children, there was inefficient information processing associated with bilateral presentation, as 
expected (Prediction 4). This finding supports the view that immaturity in the corpus callosum results in inefficient interhemispheric communication (Galin, Diamond, \& Herron, 1977; Galin, Johnstone, Nakel, Herron, 1979; Quinn \& Geffen, 1986). Like the YA group, the DD group also performed more slowly in the Bilateral RVF Condition than in the Unilateral RVF Condition $(\Delta=62.3 \mathrm{msec})$, although this effect did not reach statistical significance. Interestingly, the DD and YA groups performed similarly across the conditions; in particular, both groups performed significantly more slowly than the CA group in the Bilateral RVF Condition. These similar performance patterns between the DD and YA groups suggest that the difference between the Bilateral RVF and the Unilateral RVF Conditions demonstrated by the DD group might reflect inefficient callosal information processing (Prediction 5). This finding supports the developmental delay model of DD (Beech \& Harding, 1984; Bruck, 1988; Treiman \&Hirsh-Pasek, 1985; Vellutino \& Scanlon, 1987). Despite the fact that the DD and CAP groups demonstrated the similar response patterns in the Unilateral RVF Condition, they demonstrated clearly different performance patterns in the Bilateral RVF Condition, i.e., slower responses for the DD group and faster responses for the CAP group.

Taken together, in the Bilateral RVF Condition, the CAP group processed information more efficiently in the bilateral than the unilateral presentation, whereas the DD and YA groups were less efficient in the bilateral than the unilateral presentation. These opposite performance patterns qualitatively dissociated the DD group from the CAP group. That is, the reading problems of the DD group might stem from inefficient interhemispheric communication, whereas the CAP group demonstrated efficient interhemispheric communication. This suggests that their reading problems do not stem 
from the same factor, at least in terms of the genu. This finding supported the hypothesis that DD and poor reading ability qualitatively differ (Prediction 7).

Because the DD group demonstrated poor performance in this phonological task, the present findings support the phonological deficit theory. Since poor performance was demonstrated in the linguistic task in the Bilateral RVF Condition, it could be argued that dysfunction in the LH could cause this poor performance. However, if dysfunction in the LH was responsible for the poor performance, the DD group should have performed more poorly than the YA and CAP groups in the Unilateral RVF Condition since they have an intact LH. However, this was not the case. Thus, it was unlikely that dysfunction in the $\mathrm{LH}$ addressed by the left hemisphere dysfunction theory was responsible for the DD group's slow performance in the Bilateral RVF Condition.

The RVF superiority effect suggests that the LH was primarily responsible for phonological processing in this task. There was no significant finding associated with the LVF Conditions, the discussion focused on both Bilateral and Unilateral RVF Conditions.

Overall, the standardized reading test scores (SWE and PDE, sub-tests of the TOWRE) correlated with the RT scores negatively and the accuracy scores positively in the Bilateral and Unilateral Conditions (Prediction 6). This suggested that reading is related to both intra- and interhemispheric communication. This supports the notion in the interhemispheric disconnection theory that reading involves multiple brain systems in the two cerebral hemispheres.

In summary, the $\mathrm{DD}$ group performed similarly to the YA group on a phonologically challenging task and dissimilarly to the CA and CAP groups. The data for 
the YA group (mean age of 9.01 years) suggested inefficient callosal information processing. The DD group's similar performance to the YA group suggested that their slower responses on the Bilateral Condition might be due to inefficient interhemispheric communication. This supports the view that DD is associated with inefficient interhemispheric communication. The quite different performance patterns in the Bilateral RVF Condition between the DD and CAP groups suggest that their reading problems stem from different factors and that DD is qualitatively different from general poor reading ability. The findings of the correlation between the SWE and PDE scores and phonological judgment in both the Bilateral and the Unilateral RVF Conditions suggest that intrahemispheric as well as interhemispheric communication are involved in phonological processing. This supports the notion that reading requires multiple brain systems.

In conclusion, Experiment 2 generally supported the interhemispheric disconnection theory of DD. The findings also supported the phonological deficit theory but did not support the left hemisphere dysfunction theory. DD appears to be a specific reading disorder, as the DD group was distinct from the CAP group at least in phonological processing. 


\section{Chapter 12: Experiment 3}

The aim of Experiment 3 was to investigate further the involvement of interhemispheric communication using a primed lexical decision task (LDT) in a visualhalf field paradigm. As noted, semantic information processing is believed to be processed through the isthmus (corresponding to the $\mathrm{CC} \# 6$ in Figure 5.2). Although the rationale of Experiment 3 was fully discussed in Chapters 8.3.3, four points should be highlighted in formulating specific predictions for the interhemispheric inefficiency hypothesis and the specific reading disorder hypothesis. First, priming effects (facilitation effects) are believed to reflect the strength of word relatedness in the mental lexicon. Second, previous studies (Zaidel, 1978, 1982; Burgess \& Simpson, 1988; Chiarello, 1985, Chiarello et al., 1990; Chiarello \& Richards, 1992; Clarke \& Zaidel, 1994; Koivisto \& Laine, 2000) suggest that both the LH and the RH are capable of processing semantic information. Third, information processing using both hemispheres is more efficient than one hemisphere for some complex tasks (cf. Banich \& Belger; Belger \& Banich, 1992). Finally, semantic processing is an important component of reading (Adams, 1991). Based on these points, the following predictions were formulated with regard to the expected performance of the four groups (DD, CA, CAP, YA) in the primed LDT:

Prediction 1: The RVF superiority effect would be observed in all four groups because the LH is primarily specialized for linguistic information processing.

Prediction 2: Both LH and RH show priming effects in all four groups (Chiarello, 1985; Clarke \& Zaidel, 1994). 
Prediction 3: The CA group would respond the fastest and most accurately among the groups across the conditions. The DD group would respond the slowest and least accurately among the groups due to their reading age.

Prediction 4: The CA and CAP groups would respond faster and more accurately in the Bilateral RVF Condition than in the Unilateral RVF Condition due to a combination of efficient information processing in the bilateral presentations and linguistic specialization of the RVF.

Prediction 5: The DD group would respond more slowly and less accurately in the Bilateral RVF Condition than in the Unilateral Condition due to inefficient information processing in the bilateral presentations and linguistic specialization of the RVF.

Prediction 6: The YA group would respond more slowly and less accurately in the Bilateral RVF Condition than the Unilateral RVF Condition due to inefficient information processing in the bilateral presentations and linguistic specialization of the RVF.

Prediction 7: The response of the DD group to the nonword stimuli would be the slowest and least accurate among the groups due to poor phonological processing (Rack et al., 1992; Snowling, 1995, 2000).

Prediction 8: The TOWRE scores would be negatively correlated with RT and positively correlated with accuracy scores in the Bilateral Condition only if interhemispheric communication is associated with reading processing.

Prediction 9: If DD is qualitatively distinct from poor reading ability, the DD group would show different patterns of performance from the CAP group. 


\subsection{Method}

\subsubsection{Apparatus}

The same apparatus described in Experiment 2 was used for Experiment 3.

\subsubsection{Stimulus Materials}

The four stimulus lists are reproduced in Appendix P. Three types of stimulus pairs were incorporated in each list: associated, non-associated, and nonword. For the associated pairs, associated primes and targets were chosen from the Norms of Word Association (Postman \& Keppel, 1970). For the non-associated pairs, two words which have little or no association were coupled (e.g., purse-clip). The nonwords were made from the associated or non-associated words by changing an initial consonant of these words to an orthographically dissimilar consonant (e.g., tirl from girl). These nonwords were coupled with a real word (e.g., wolf-tirl). All words and nonwords had three to six letters with one or two syllables. A different stimulus list was generated for each Condition, i.e., the Bilateral RVF, Unilateral RVF, Bilateral LVF, and the Unilateral LVF Conditions. Each list contains 15 associated pairs, 15 non-associated pairs, and 15 word-nonword pairs. The total number of stimulus pairs was 45 . There were four stimuli lists in total, each of which was equated in terms of the word frequency (Francis \& Kucera, 1982), the strength of association (Postman \& Keppel, 1970), and the letter length of targets. A pilot study was conducted to examine priming effects with the stimulus lists using the same equipment and experimental procedures with 20 righthanded male university students, who participated in the pilot study towards a credit in 
introductory psychology. The results showed that the associated pairs were responded to significantly faster and more accurately than the non-associated pairs. This indicates the presence of priming effects and validated the present stimulus lists. The pilot data are summarized in Appendix Q.

\subsubsection{Procedure}

The procedure was nearly identical to that of Experiment 2 except for the stimulus types and duration of stimulus presentation. The participant was seated on a chair in front of a computer monitor. The procedure was illustrated on an information sheet with examples of stimuli (two examples of a word and a nonword). While the participant viewed the illustration, the procedure and examples were explained. However, the participant was not told what types of pairs would be presented. The participant was instructed to place his or her chin on the chin-rest, forehead against the head-rest, and to look straight ahead at the dot throughout the trial. The participant was also instructed to guess if he or she was not sure of the answer. All stimuli were presented in Courier New 13 font size in black print on a white screen and projected at $2.5^{\circ}$ horizontally to the left or right of the center fixation dot (font size 40 ) on the monitor. Thus, even if small eye movements occurred, projection of the image to the opposite hemisphere was ensured (Banich, 1997). In every trial, the total word length was located between $2.5^{\circ}$ and $1.5^{\circ}$ from the center fixation dot (Brysbaert \& $D^{\prime} Y$ dewalle, 1990; Fuentes \& Tudela, 1992). 


\section{A primed Lexical Decision Task}

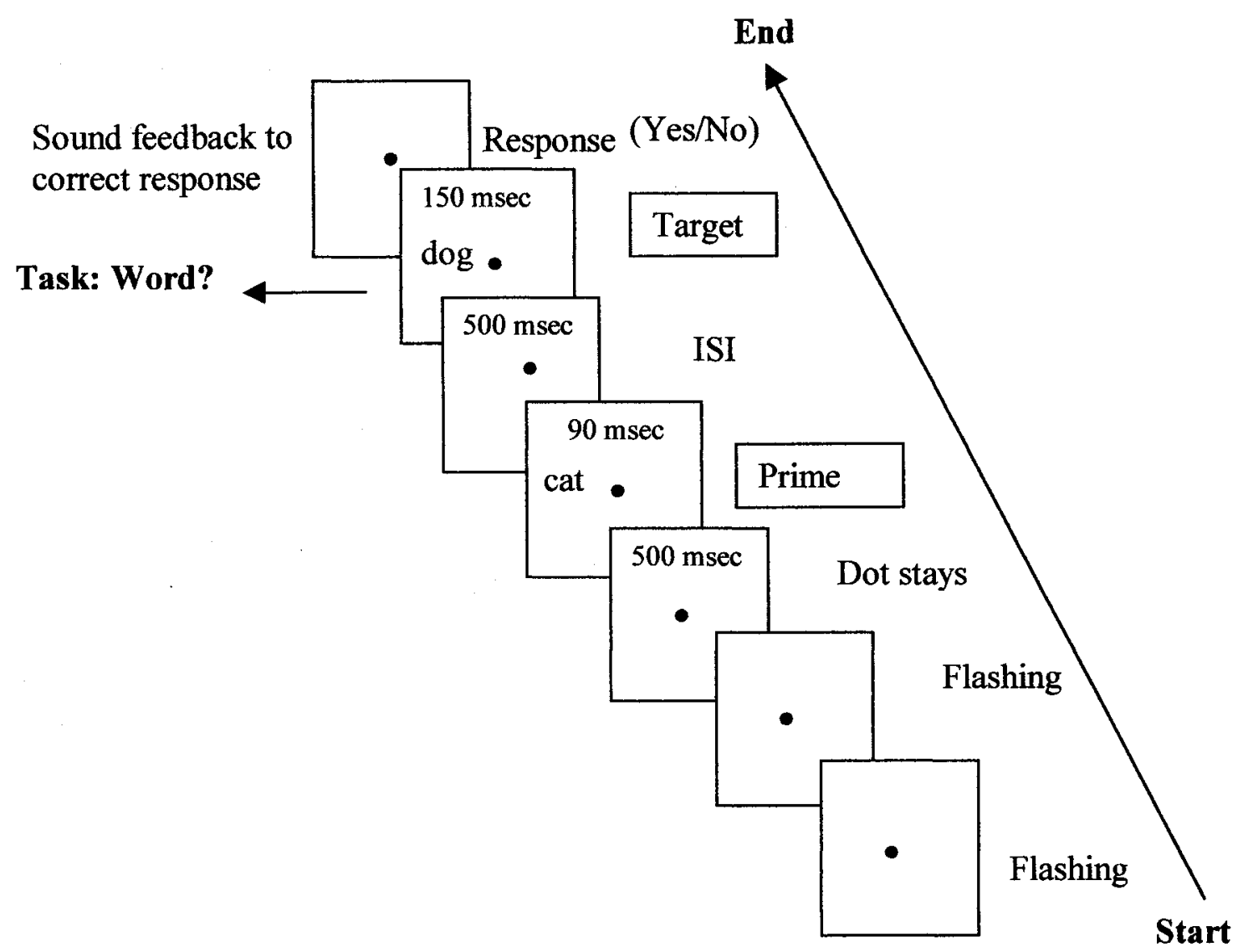

Figure 12.1. Procedure for the lexical decision task.

The dot flashes twice, and then stays in the center. The prime word appears for $90 \mathrm{msec}$ followed by a 500msec ISI (interstimulus interval). The target (either a word or nonword) then appears for $150 \mathrm{msec}$ in the same visual field for the Unilateral Condition and on the opposite visual field for the Bilateral Condition. The participant makes a judgment as to whether the target is a word.

As shown in Figure 12.1, at the beginning of the trial, a fixation dot flashed twice in the center of the monitor as a starting signal. The third fixation dot then appeared and stayed in the center throughout the trial. As in Experiment 2, the visual half-field presentation procedure requires participants to focus on the center during the trial, and 
thus, the fixation dot was necessary to minimize eye movements (Banich, 1997). 500msec after the third fixation dot appeared on the screen, the prime (always a word) was presented in either the RVF/LH or the LVF/RH for $90 \mathrm{msec}$. The participant had previously been instructed to ignore the first word. The target (either a word or nonword) was sequentially presented in the same VF (Unilateral Condition) for $150 \mathrm{msec}$. This duration for the prime and target has been used in previous studies with university students (Clarke \& Zaidel, 1994) and DD children (Brysbaert \& D'Ydewalle, 1990). For the Bilateral Condition, the prime was presented in either the RVF/LH or the LVF/RH, and the target was sequentially presented on the opposite VF. The interval between prime and target (interstimulus interval, ISI) was $500 \mathrm{msec}$, as in Experiment 2. Reaction time (RT) and correct responses to the target were recorded by computer.

Four conditions were completed by each participant (i.e., Unilateral LVF, Bilateral LVF, Unilateral RVF, and Bilateral RVF, where VF indicates the target VF). At the beginning of each condition, the participant was instructed on which index finger was to be used to click the mouse for response. When the right index finger was used, 45 test pairs were presented in the RVF and 12 filler pairs were presented in the LVF. The RT of the fillers was not included in the subsequent analyses. When the left index finger was used, the 45 test pairs presented in the LVF and 12 filler pairs were presented RVF. This manipulation was the same in the Unilateral and Bilateral Conditions, and was necessary to prevent the participant's expectation that a stimulus was coming from one VF.

The participant was instructed to respond to the target by clicking a mouse, labeled with 'Yes' (right side) for a word and 'No' (left side) for a nonword, with an index finger, as quickly and accurately as possible. There was no time limit for the response; 
however, if the participant did not respond after more than five seconds, he or she was encouraged to respond by the experimenter. A total of 12 practice trials was provided, followed by 57 trials ( 45 test trials and 12 fillers) with auditory feedback (i.e., happy sound) for the correct responses. The order of the four stimulus lists and conditions was counterbalanced across the participants. The order of stimuli in each list was randomized by computer for each participant.

\subsubsection{Analysis}

Reaction time and accuracy were the dependent variables. The accuracy (i.e., number of correct responses) data were transformed to percentages for the analysis to compare the present study with a previous study using percentage data (Clarke \& Zaidel, 1994).

A 4 (Group: CA, CAP, DD, YA) 2 (Visual Field: right, left) $\times 2$ (Condition: bilateral, unilateral) $\times 3$ (Stimulus Type: associated, non-associated, nonword) MANOVA with mixed repeated-measures was used. Group was a between-groups factor and Visual Field, Condition and Stimulus Type were within-group factors. Significant effects were subsequently investigated using the Bonferroni corrected $\alpha$ for pairwise comparisons. As in Experiment 1 and 2, the Bonferroni test divided $\alpha$ level (.05) by the number of comparisons to ensure avoiding Type I errors (Stevens, 1996).

To investigate Prediction 8, correlation analyses were separately conducted on the $\mathrm{RT}$ and percentage of correct responses in the associated, non-associated and nonword pairs of the Unilateral LVF and RVF, and the Bilateral LVF and RVF Conditions with age, TOWRE scores and TONI-3 scores. 


\subsection{Results}

As in Experiments 1 and 2, preliminary analyses were conducted on the data. The Box plot test (Stevens, 1996) showed that the box line did not reach either edge of the box in all observations, suggesting that there was no ceiling and floor effect in the data. The Box plot test identified extreme values in the data. The Shapiro-Wilk test for normality indicated that the MANOVA assumption of normality was not met. The Levene test for the homogeneity of variances for the groups revealed that the assumption of the homogeneity of variance was not met. To solve the extreme values and the violation of the assumptions, as in Experiment 2, it was decided to use the median from individual scores to calculate group means (Stevens, 1996). The group means of RT and the percentage of correct response are presented in Table 12.1.

Another preliminary MANOVA was performed to examine the possible group differences on Gender and Handedness variables. There were no significant main effects and interactions with Group, Condition, Visual Field, and Stimulus Type. Therefore, these data were pooled across Gender and Handedness.

For the main analysis, a 4 (Group: CA, CAP, DD, YA) x 2 (Condition: bilateral, unilateral) $\times 2$ (Visual Field: right, left) x 3 (Stimulus Type: associated, non-associated, nonword) MANOVA with mixed repeated measures was performed on the group mean of RT and the percentage of correct responses. There was no main effect or interaction on Condition, indicating that this independent variable had no effect on the two DVs. Subsequently, the data were reanalyzed collapsing across the Condition variable. The reanalysis revealed a two-way interaction of Group x Stimulus Type, $F 9(12,357)=2.21$, 
$p<.011, \mathrm{y}^{2}=.061$, and three significant main effects: Visual Field, $F(2,137)=35.54, p$ $<.000, \mathrm{y}^{2}=.342$, Stimulus Type, $F(4,135)=62.88, p<.000, \mathrm{\eta}^{2}=.651$, and Group, $F(6$, 274) $=9.20, p<.000, \mathrm{n}^{2}=.168$.

Table 12.1

Group Means of Reaction Time and Percentage of Correct Responses

\begin{tabular}{|c|c|c|c|c|c|c|c|c|c|}
\hline & & & RT & (msec) & & & Correct & $(\%)$ & \\
\hline Group & $\begin{array}{c}\text { Stimulus } \\
\text { type }\end{array}$ & $\begin{array}{l}\text { LVF } \\
(S D) \\
\end{array}$ & $\begin{array}{c}\text { BLVF } \\
(S D) \\
\end{array}$ & $\begin{array}{l}\text { RVF } \\
(S D) \\
\end{array}$ & $\begin{array}{c}\text { BRVF } \\
(S D) \\
\end{array}$ & $\begin{array}{l}\text { LVF } \\
(S D) \\
\end{array}$ & $\begin{array}{c}\text { BLVF } \\
(S D) \\
\end{array}$ & $\begin{array}{l}\mathrm{RVF} \\
(S D) \\
\end{array}$ & $\begin{array}{c}\text { BRVF } \\
(S D) \\
\end{array}$ \\
\hline DD & $\begin{array}{r}\text { Asso } \\
\text { Non-Asso } \\
\text { Nonword }\end{array}$ & $\begin{array}{l}1073.2 \\
(288.6) \\
1068.3 \\
(393.5) \\
1247.4 \\
(433.2)\end{array}$ & $\begin{array}{l}1048.9 \\
(259.4) \\
1142.8 \\
(410.5) \\
1281.3 \\
(433.3)\end{array}$ & $\begin{array}{c}961.1 \\
(233.0) \\
1003.3 \\
(277.3) \\
1341.8 \\
(625.5)\end{array}$ & $\begin{array}{c}952.3 \\
(267.4) \\
1002.8 \\
(260.0) \\
1293.8 \\
(386.4)\end{array}$ & $\begin{array}{c}79.6 \\
(12.3) \\
64.9 \\
(19.2) \\
54.0 \\
(18.7)\end{array}$ & $\begin{array}{c}76.1 \\
(17.0) \\
66.7 \\
(14.1) \\
49.1 \\
(18.4)\end{array}$ & $\begin{array}{c}83.9 \\
(16.2) \\
74.4 \\
(15.0) \\
62.8 \\
(15.1)\end{array}$ & $\begin{array}{c}83.5 \\
(17.0) \\
74.0 \\
(16.3) \\
55.4 \\
(19.1)\end{array}$ \\
\hline CA & $\begin{array}{r}\text { Asso } \\
\text { Non-Asso } \\
\text { Nonword }\end{array}$ & $\begin{array}{c}805.4 \\
(171.2) \\
844.2 \\
(190.1) \\
1014.4 \\
(213.1)\end{array}$ & $\begin{array}{c}793.8 \\
195.0) \\
845.6 \\
(188.9) \\
986.9 \\
(223.9)\end{array}$ & $\begin{array}{c}773.8 \\
(162.8) \\
844.2 \\
190.1) \\
1014.4 \\
(213.1)\end{array}$ & $\begin{array}{c}752.8 \\
(172.4) \\
796.2 \\
(186.7) \\
922.0 \\
(175.7)\end{array}$ & $\begin{array}{c}82.1 \\
(14.4) \\
80.5 \\
(15.7) \\
70.4 \\
(21.1)\end{array}$ & $\begin{array}{c}86.7 \\
(12.5) \\
76.8 \\
(19.6) \\
68.3 \\
(20.9)\end{array}$ & $\begin{array}{c}90.0 \\
(8.6) \\
86.9 \\
(11.8) \\
74.5 \\
(22.4)\end{array}$ & $\begin{array}{c}88.3 \\
(13.0) \\
84.1 \\
15.4) \\
71.7 \\
(22.4)\end{array}$ \\
\hline CAP & $\begin{array}{r}\text { Asso } \\
\text { Non-Asso } \\
\text { Nonword }\end{array}$ & $\begin{array}{c}953.3 \\
(441.5) \\
1005.9 \\
(515.6) \\
1110.2 \\
(565.8)\end{array}$ & $\begin{array}{c}947.0 \\
(409) \\
907.4 \\
(322.9) \\
1279.9( \\
596.3)\end{array}$ & $\begin{array}{c}906.0 \\
(557.6) \\
917.1 \\
545.7) \\
986.1 \\
(320.1)\end{array}$ & $\begin{array}{c}924.3 \\
(453.3) \\
939.8 \\
(468.7) \\
1080.3 \\
(415.5)\end{array}$ & $\begin{array}{c}73.3 \\
(18.6) \\
70.0 \\
(16.4) \\
50.0 \\
(19.4)\end{array}$ & $\begin{array}{c}80.7 \\
(14.2) \\
72.7 \\
(11.1) \\
52.7 \\
(27.5)\end{array}$ & $\begin{array}{c}87.3 \\
(9.1) \\
78.0 \\
(8.9) \\
64.7 \\
(16.3)\end{array}$ & $\begin{array}{c}88.7 \\
(8.9) \\
79.3 \\
(12.7) \\
67.3 \\
(24.2)\end{array}$ \\
\hline YA & $\begin{array}{r}\text { Asso } \\
\text { Non-Asso } \\
\text { Nonword }\end{array}$ & $\begin{array}{l}1011.5 \\
(339.0) \\
1066.9 \\
(326.3) \\
1282.8 \\
(441.3)\end{array}$ & $\begin{array}{c}996.7 \\
(266.4) \\
1044.6 \\
(310.9) \\
1210.8 \\
(402.8)\end{array}$ & $\begin{array}{c}940.6 \\
(328.0) \\
999.3 \\
(397.9) \\
1220.8 \\
(397.2)\end{array}$ & $\begin{array}{c}948.8 \\
(264.0) \\
945.3 \\
(225.7) \\
1219.8 \\
(382.5)\end{array}$ & $\begin{array}{c}73.1 \\
(19.3) \\
71.2 \\
(20.3) \\
62.7 \\
(26.0)\end{array}$ & $\begin{array}{c}74.2 \\
(18.0) \\
67.8 \\
(16.1) \\
61.6 \\
(22.1)\end{array}$ & $\begin{array}{c}78.7 \\
(17.2) \\
77.1 \\
(12.4) \\
62.6 \\
(25.0)\end{array}$ & $\begin{array}{c}77.5 \\
(20.3) \\
77.0 \\
(17.6) \\
61.5 \\
(23.4)\end{array}$ \\
\hline
\end{tabular}

Note: Asso = Associated type of stimuli; Non-Asso = Non-associated type of stimuli; Nonword $=$ Nonword stimuli; $B R V F=$ Bilateral right visual field; $R V F=$ Right visual field; $B L V F=$ Bilateral left visual field; $L V F=$ Left visual field; $\mathrm{LH}=$ Left Hemisphere; RH: Right hemisphere.

To investigate the between-group factor in the two-way interaction, pairwise comparisons were conducted. They revealed that the DD group $(M=1008.89 \mathrm{msec})$ responded significantly more slowly than the CA group $(M=782.51 \mathrm{msec})$ in the 
associated pairs, $p=.000$. The DD group $(M=1054.91 \mathrm{msec})$ also demonstrated significantly slower responses than the CA group $(M=830.80 \mathrm{msec})$ in the nonassociated pairs. Unexpectedly, the DD group did not significantly differ in RT from the CAP group $(M=932.60 \mathrm{msec})$ and the YA group $(M=976.48 \mathrm{msec})$ in both the associated pairs and the non-associated pairs (CAP: $M=942.53 \mathrm{msec}$, YA: $M=1012.90$ msec). However, the YA group responded significantly more slowly than the CA group in the associated word pairs, $p=.001$, and the non-associated word pairs, $p=.005$. The CAP group did not significantly differ from the other three groups in both stimulus types. The data are summarized in Figure 12.2.

Regarding the critical nonword pairs, the DD group $(M=1291.09 \mathrm{msec})$ responded significantly more slowly than the CA group $(M=966.47), p=.000$, and in fact, showed the slowest response among all the groups (CAP: $M=1114.09$ msec; YA: $M$ $=1208.16 \mathrm{msec})$. However, the observed differences among the DD, CAP, and YA groups were not statistically supported. The CA group responded significantly faster than the YA group, $p=.002$, but did not significantly differ from the CAP group. 


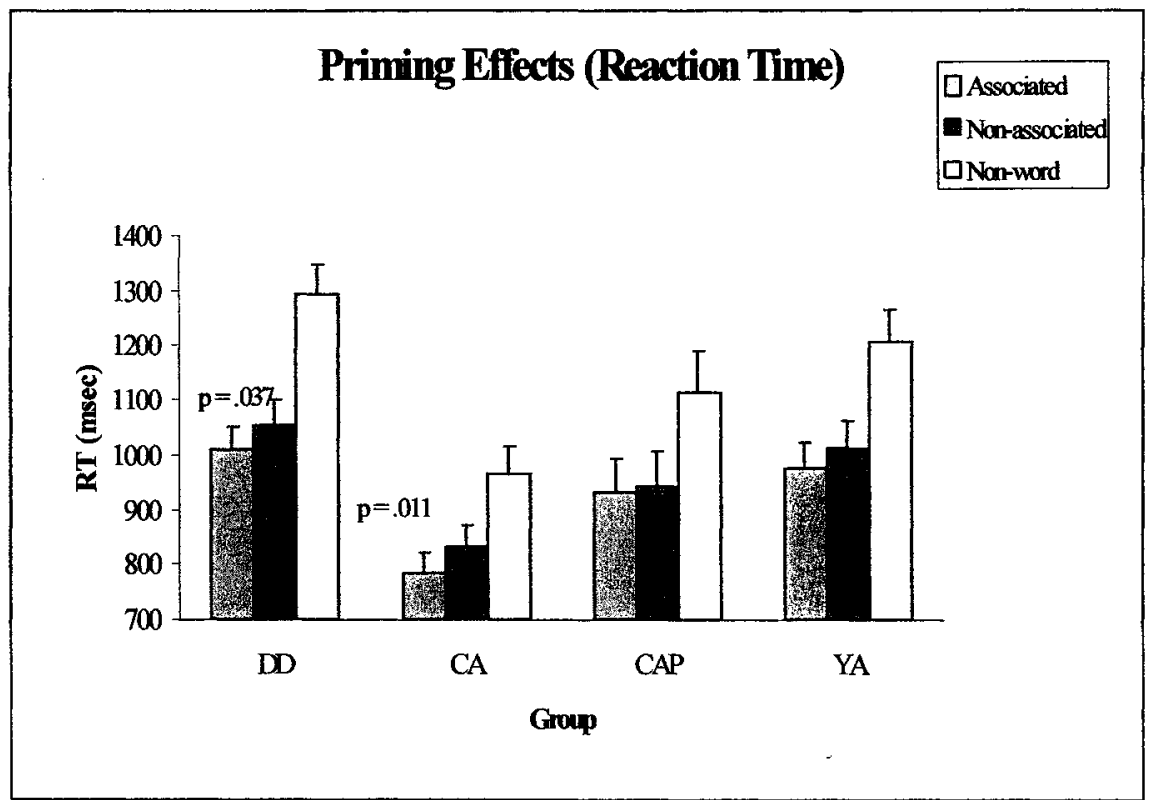

Figure 12.2. The interaction of Group x Stimulus Pair in RT.

Associated = Associated word pairs; Non-associated = Non-associated word pairs;

Nonword $=$ Nonword pairs.

When the accuracy data were investigated, pairwise comparisons revealed the DD group ( $M=70.00 \%)$ responded significantly less accurately than the CA group $(M=$ $82.07 \%)$ in the non-associated pairs, $p=000$. The DD group $(M=55.35 \%)$ also showed significantly less accurate performance than the CA group $(M=71.47 \%)$ in the nonword pairs, $p=.002$. The YA group $(M=72.60 \%)$ significantly differed from the CA group in the associated pairs, $p=.000$, although this group did not differ from the DD group across all the stimulus types. The CAP group did not differ from any groups across the stimulus types. 


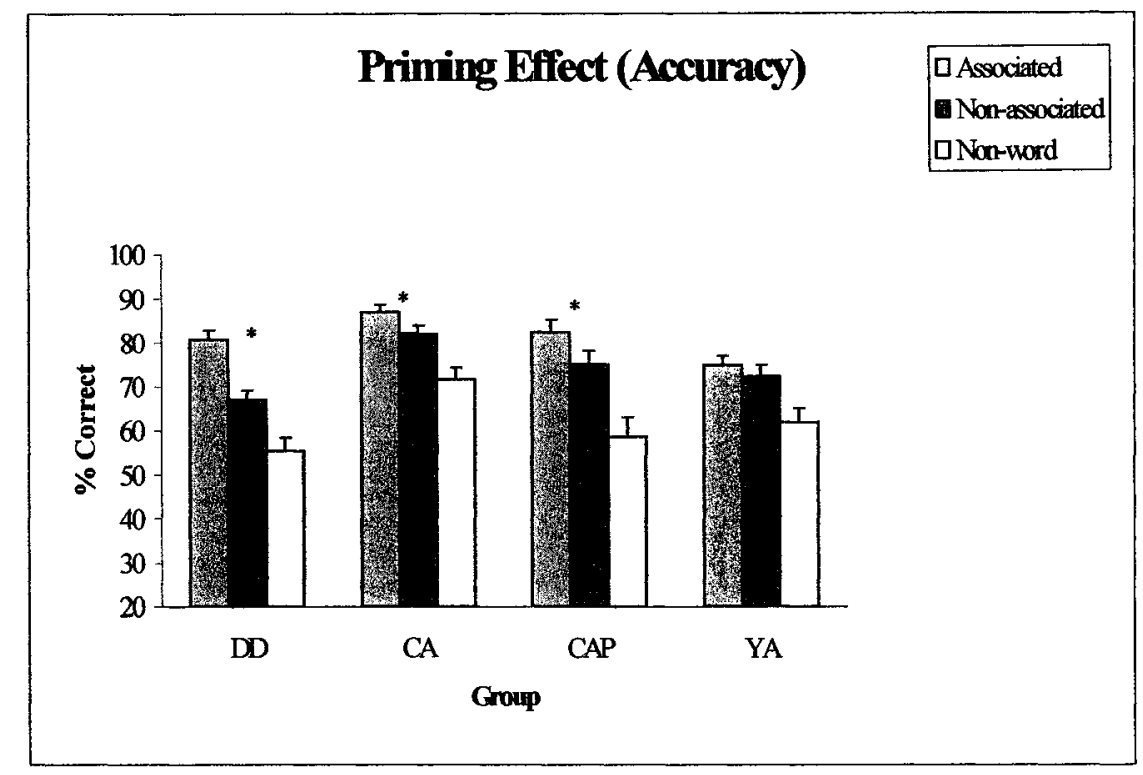

Figure 12.3. The interaction of Group $x$ Stimulus Type in accuracy.

Associated: Associated word pairs. Non-associated: Non-associated word pairs.

Nonword: Nonword pairs.

${ }^{*} p<.004$.

To investigate the within-group factor, pairwise comparisons were conducted. They revealed that the DD group responded to the associated pairs faster than the nonassociated pairs, $p=.037(\Delta=45.42 \mathrm{msec})$. The CA group also responded to the associated pairs faster than the non-associated pairs $(\Delta=48.29 \mathrm{msec}), p=.011$, although these differences were trends. When the accuracy data were investigated, the DD group $(\Delta=10.8 \%)$ demonstrated a significant difference between the associated and nonassociated stimulus pairs, $p=.000$. The significant differences between these stimulus types were found in the CA group $(\Delta=4.9 \%), p=.003$, as well as in the CAP group ( $\Delta=$ $7.5 \%), p=.004$. The data are summarized in Figures 12.2 and 12.3 . 
The significant main effect of Visual Field and the absence of interaction of Visual Field $x$ Group showed that the target presented in the RVF $(M=980.1 \mathrm{msec}, M=$ $76.1 \%$ correct) was responded to faster and more accurately than the target presented in the LVF $(M=1040.1 \mathrm{msec} ; M=69.2 \%$ correct $)$ across the groups. This confirmed the presence of the RVF superiority effect across the groups.

The significant main effect of Stimulus Type and the absence of interaction of Stimulus Type $\mathrm{x}$ Visual Field $\mathrm{x}$ Group showed that both hemispheres processed all the stimulus pairs in a similar manner across the groups. That is, all groups responded faster $(M=925.10 \mathrm{msec})$ and more accurately $(M=81.3 \%)$ to the associated pairs than to the non-associated pairs $(M=960.13 \mathrm{msec} ; M=74.9 \%)$, and to the nonword pairs $(M=$ $1145.0 \mathrm{msec} ; M=61.8 \%$ ) across the VFs. Since both the RH and the LH obtained priming effects, this confirmed that both cerebral hemispheres are capable of processing semantic information.

Finally, Pearson product moment correlations were calculated between age, the non-verbal IQ scores (TONI-3), standardized test scores (TOWRE), RT and percentage of correct response of DVs reflecting critical results of the primed LDT. Significant positive correlations were observed between TOWRE scores and these DVs in the Bilateral and Unilateral Conditions. 
Table 12.2

Correlations of Semantic Task Scores with Age, TONI-3, and TOWRE Scores

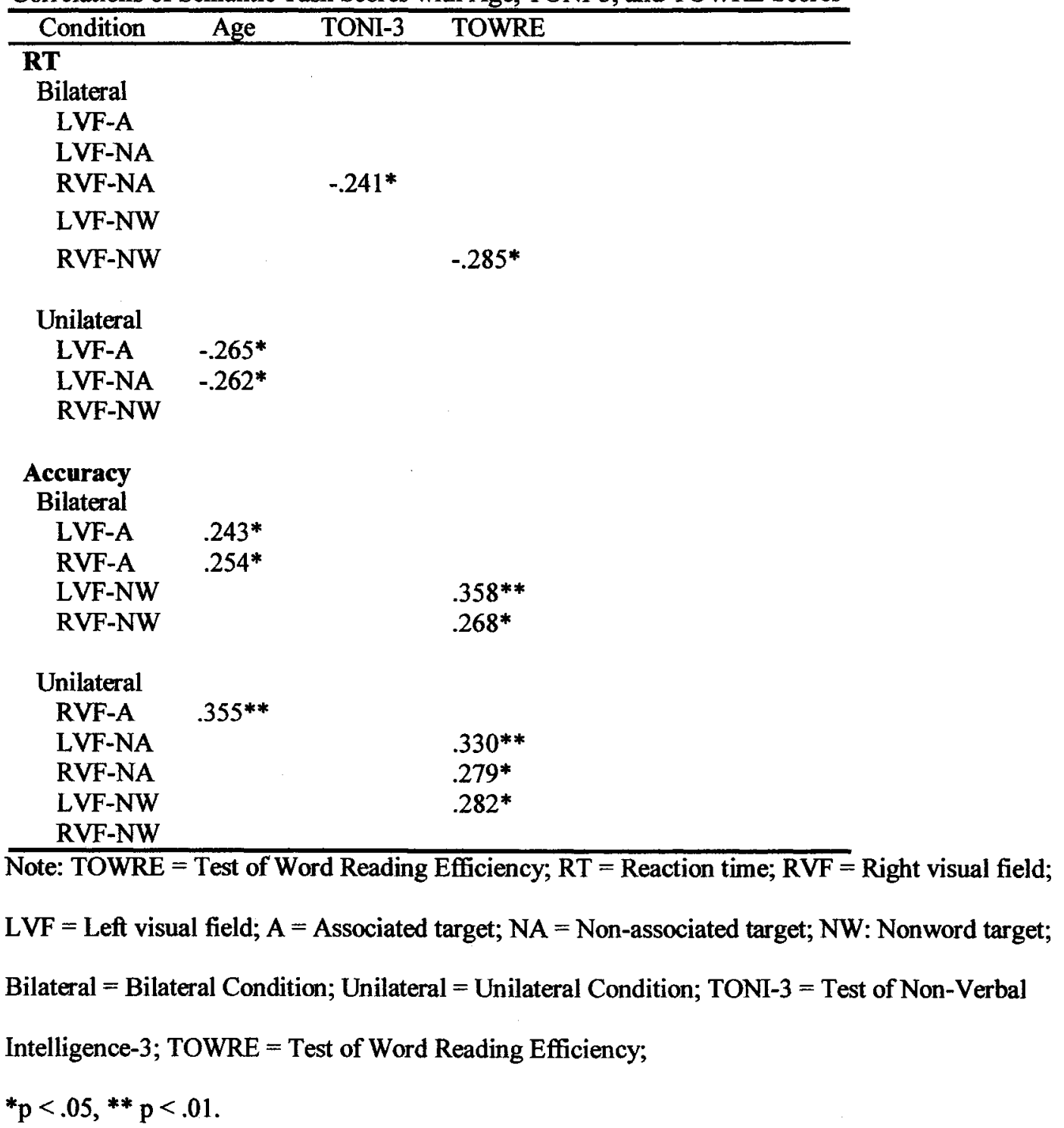




\subsection{Discussion}

The principal aim of this experiment was to investigate the involvement of interhemispheric communication in DD associated with semantic processing using a primed LDT. The main significant findings were: (1) the RVF superiority was observed in all four groups; (2) the DD group responded significantly more slowly and less accurately than the CA group to all the stimulus types; (3) the YA group demonstrated significantly slower and less accurate responses than the CA group in the associated and non-associated pairs; (4) priming effects were observed in both VFs; (5) priming effects in accuracy were observed in the DD, CAP, and CA groups; (6) priming effects in RT were observed in the DD and CA groups; and (7) the TOWRE scores correlated with the RT and accuracy scores of the task in both the Bilateral and Unilateral Conditions.

Unexpectedly, there was no difference of responses between the Bilateral and the Unilateral Conditions in all four groups. This means that information was processed similarly in both the Conditions. These results were consistent with Clarke and Zaidel's study (1994) and our pilot study (see Appendix Q) with normal university participants. Alternately, Clarke and Zaidel speculated on the lack of interaction of Condition $\mathbf{x}$ Visual Field such that semantic information was transferred to the opposite hemisphere during the 500-msec delay, no matter in which VF the target was presented, i.e., either the RVF or the LVF. According to this interpretation, the absence of a main effect of Condition and interaction of Condition $\mathrm{x}$ Visual $\mathrm{x}$ Group in the present study suggests that semantic information was transferred to the opposite hemisphere during the 500-msec ISI in the 
DD group as was the case in their control groups. There was no evidence of inefficient interhemispheric communication in the for this semantic task or at least with this ISI.

A possible explanation for the failure to support the hypothesis that inefficient interhemispheric communication is associated with DD is simply not viable. However, considering the neuroimaging studies (Pugh et al., 2000; Shaywitz et al., 2002), which have shown abnormal brain activation in Wernicke's area with linguistic tasks, this is unlikely. Another possible explanation is that the small effect size $\left(\eta^{2}=.012\right)$ affected the results. As discussed in Chapter 10, Cohen (1977) characterized eta squared $\left(\mathrm{y}^{2}\right)$ such that when total sample size is about 50 or more, $\mathrm{y}^{2}=.01$ as small, $\mathrm{y}^{2}=.06$ as medium, and $\eta^{2}=.14$ as a large effect size. Based on Cohen's classification, the effect size $\left(\eta^{2}=\right.$ .012 ) in the present study was characterized as small. That is, the long ISI (i.e., 500 msec) used in the task may have made the task relatively easy for all the participants including the DD children, if it gave them enough time to complete processing the prime before the target was input into the system. Thus, the prime and target could have been processed on a one by one basis, whether the participant was a good reader or not.

At any rate, the present data did not support the hypothesis that DD is associated with inefficiency in interhemispheric communication affecting semantic processing (Prediction 5). Neither did it support the prediction that efficiency in interhemispheric communication of semantic processing for the CA and CAP groups (Prediction 4) nor the prediction that inefficiency in interhemispheric communication for the YA group (Prediction 6). It also did not support Prediction 8 that the TOWRE scores would be more correlated with the RT and accuracy in the Bilateral RVF than in the Unilateral RVF. 
As expected, the RVF superiority effect was observed across all the groups (Prediction 1). Targets presented in the RVF were responded to faster and more accurately than those presented in the LVF. This is compatible with the view that the LH is more specialized for the processing of linguistic information than the $\mathrm{RH}$. The finding that the RVF superiority effect was observed in the DD group does not support the left hemisphere dysfunction theory of DD. In addition, the significant main effect of Stimulus Type in the absence of Stimulus Type $\mathrm{x}$ Visual Field X Group interaction indicates that both hemispheres are capable of processing semantic information (Chiarello, 1985; Koivisto \& Laine, 2000) in all the groups. This finding was expected (Prediction 2). As expected (see Figure $12.2 \& 12.3$ ), the CA group responded to the associated and non-associated word pairs (i.e., lexical judgment) faster and more accurately than the DD, CAP and YA groups (Prediction 3), although the difference between the CA and CAP groups was not statistically significant. Interestingly, the DD, CAP, and YA groups did not demonstrate a significant difference in all stimulus types. The similar performance between the DD and YA groups could be explained by a developmental delay model, as discussed in Experiment 1. The patterns of response demonstrated by the CAP group suggest that semantic processing is associated with both reading ability and age-related components independent of non-verbal IQ, or perhaps a combination of both.

As expected, the DD group responded to the nonwords (i.e., non-lexical judgment) more slowly and less accurately than the CA group (Prediction 7). Because non-lexical judgment requires phonological processing, these findings confirmed that DD children have problems in phonological processing as the literature had suggested (e.g., Rack et al., 1992; Snowling, 1995, 2000). More importantly, the DD group was not 
significantly slower or less accurate than the CAP and YA groups in this non-lexical judgment task. This finding suggests that poor phonological processing was also a problem for the CAP group. In other word, poor phonological processing is not distinct for DD; rather, it appears to be a common characteristic in both DD and poor reading ability. This supports Stanovich's argument (Stanovich, 1991; Stanovich \& Siegel, 1994) that DD lies at the extreme edge of poor reading ability because high IQ poor readers (i.e., DD children) and low IQ poor readers (i.e., CAP readers) do not differ in terms of reading nonwords. Thus, Prediction 7 was partially supported. The implication of this finding is that nonword reading alone is not a sufficient measure to differentiate DD from poor reading ability.

The main object of this experiment was to investigate priming effects in RT and accuracy. The DD group responded significantly more accurately to the associated pairs than the non-associated pairs. This finding suggests that this group obtained priming effects (10.8\%). The CAP (7.5\%) and CA groups (4.9\%) also obtained priming effects, whereas the YA group did not. Priming effects in both the accuracy and RT data suggest good semantic connections in the mental lexicon (Anderson, 1976; Collins \& Loftus, 1975; Collins \& Quillian, 1969; Neely, 1991). Thus, the demonstration of priming effects by the DD and CA groups indicate that their semantic connections in the mental lexicon were good. In contrast, because the CAP group demonstrated priming effects only in accuracy, this effect might have resulted from a speed-accuracy trade-off. This suggests that their semantic connections were not as good as that of the DD and CA groups. These findings were compatible with those of a previous study (Swan \& Goswami, 1997). Good semantic connections imply that the reading problems of DD children are not 
likely to lie in encoding processes, but are likely to lie in the retrieval processes. As Swan and Goswami argued, poor working memory in the DD group (Breznitz, 1997; Gathercole \& Baddeley, 1990; Kramer et al., 2000; Macaruso et al., 1996; Palmer, 2000) might reflect their reading problems in word retrieval processes.

Since the CAP group did not demonstrate as good semantic connections as the DD and CA groups, they might have had problems in the encoding processes, which would reflect their impoverished semantic connections in the mental lexicon. The lack of priming effects in the YA group might be accounted for by the age of this group ( $M$ age of 9.01 years). Despite the fact their reading age ( $M$ reading age of 12.06 years) was far better than their chronological age, this group responded to the associated pairs with significantly slower responses than the CA group; in contrast, the CAP group ( $M$ reading age of 11.0 years) did not respond in this way. Thus, it is possible that their lexical network in the mental lexicon has not fully developed yet, and activation of priming was not spreading to the semantically or associatively linked words in the mental lexicon. The DD group demonstrated similar performance to the CAP and YA groups in speed and accuracy. However, the DD group's pattern of priming effects clearly dissociated them from the CAP group. This suggests that DD is qualitatively different from poor reading ability (Prediction 9).

The reading scores of the TOWRE were negatively correlated with RT scores and positively correlated with accuracy scores in the Bilateral and the Unilateral Conditions (Prediction 8). This was not expected. This suggests that reading ability is associated with both intra- and interhemispheric interaction. 
Taken together, the results of Experiment 3 suggest that the DD group processed the three types of stimuli similarly to the CAP and YA groups in terms of speed and accuracy. However, the priming effects qualitatively dissociated the DD group from the CAP and YA groups. This means that the developmental delay model, as discussed in Experiment 1, cannot provide a full explanation for the qualitative difference between the DD and YA groups. It also means that the real differences between DD and poor reading ability cannot be depicted by simply looking at poor phonological processing (i.e., nonword reading), even though this is often considered to be the main characteristic of DD. The present findings demonstrated that poor phonological processing is a common problem for DD and poor reading ability. A picture of DD will emerge only when different components, including both phonological and semantic knowledge, are taken into account.

The findings from the correlation between the standardized reading scores and both the accuracy and RT scores in the Bilateral and Unilateral Conditions were unexpected. However, they indicated that both intra- and interhemispheric communication might be related to reading ability in some ways, and support the notion that reading requires multiple brain systems.

In conclusion, Experiment 3 failed to support the interhemispheric disconnection theory. It also did not support the left hemisphere dysfunction theory due to the presence of the RVF superiority effect. Despite the fact that the pattern of scores in nonword processing did not dissociate the DD and CAP groups, intact semantic connections in the mental lexicon demonstrated by the DD group supports the view that DD is qualitatively different from general poor reading ability in at least this area. 


\section{Chapter 13: Predictors}

The aim of this chapter was to investigate which dependent variables (DVs) from the three Experiments would better predict the standardized reading scores on the TOWRE. The interhemispheric disconnection theory of DD assumes that interhemispheric information processing is required for reading (Summerfield \& Michie, 1993; Moor et al., 1996; Gladstone et al., 1989; Fabbro et al., 2001). Although previous studies (Fletcher, et a., 1982; Lindgren, 1978; Moore et al., 1996, Zung, 1986) found a correlation between reading ability and finger localization in the Crossed Condition but not in the Uncrossed Condition, in Experiment 1, the finger localization task scores were correlated with both the Crossed and the Uncrossed Conditions. The reading literature (Adams, 1991; Baron, 1979; Doctor \& Coltheart, 1980; Ehri \& Wilce, 1979; Stanovich, 1982, Stanovich et al., 1984) has suggested that phonological skills are good predictors of reading ability, particularly for the beginning readers. The poor phonological processing of DD children is generally accepted in the dyslexia literature (e.g., Rack et al., 1992; Shaywitz et al., 2000; Snowling, 1995; 2000;). In Experiment 2, the TOWRE scores were correlated with the phonological task scores in both the Bilateral and the Unilateral Conditions. The TOWRE scores were more strongly correlated with the accuracy scores than with the RT scores in both the Conditions. In Experiment 3, the TOWRE scores were also correlated more strongly with accuracy scores than with RT scores in both the Bilateral and Unilateral Conditions. Poor phonological processing is typically reflected in nonword reading. Interestingly, age was strongly correlated with the 
TOWRE scores in all three experiments. Based on previous findings and the present findings, the following predictions were formulated:

Prediction 1: The Crossed-finger localization would be strongly correlated with the TOWRE scores as assumed in the interhemispheric disconnection theory.

Prediction 2: The rhyming judgment task scores in the Bilateral RVF Condition would be strongly correlated with the TOWRE due to the phonological task, the RVF superiority, and the efficient information processing in bilateral presentations.

Prediction 3: The nonword scores in the primed LDT would be strongly correlated with the TOWRE scores due to phonological processing.

Prediction 4: The accuracy scores would be more correlated with the TOWRE scores than the RT scores in the rhyming judgment task and primed LDT. There were 12 DVs associated with the experimental conditions that might measure similar components (see Table 13.1). Thus, a Principal Component Analysis (PCA) was performed to group these variables with eigenvalues greater than 1 in each experiment of the present thesis. The PCA extracted two components of Experiment 1 (the finger localization task), which were renamed Uncrossed-FLT (finger localization task) and Crossed-FLT. For Experiment 2 (the rhyming task), two components were extracted by the PCA. These were renamed Rhyming-Accuracy and Rhyming-Speed. For Experiment 3 (the primed lexical task, PLDT), three components were extracted by the PCA. These were renamed PLDT-Word-Speed, PLDT-Word-Accuracy, and PLDTNonword-Accuracy. A regression analysis was performed on the seven new DVs 
together with age, gender and handedness to see which component would be the better predictors for the TOWRE scores.

Table 13.1

Experimental Tasks of the Three Experiments

Condition

\begin{tabular}{llll}
\hline & \multicolumn{1}{c}{ Task } & Uncrossed/Unilateral & \multicolumn{1}{c}{ Crossed/Bilateral } \\
\hline Experiment 1 & Finger Localization & 2-finger sequence & 2-finger sequence \\
& & 3-finger sequence & 3-finger sequence \\
Experiment 2 & \multirow{2}{*}{ Rhyming } & LVF & LVF \\
& & RVF & RVF \\
Experiment 3 & \multirow{2}{*}{ Primed Lexical Decision } & LVF & LVF \\
& & RVF & RVF \\
\hline
\end{tabular}

Note: Uncrossed = Uncrossed Condition; Unilateral = Unilateral Condition; Crossed = Crossed Condition;

Bilateral $=$ Bilateral Condition, $\mathrm{LVF}=$ Left visual field $; \mathrm{RVF}=$ Right visual field

The regression analysis revealed that overall multiple regression $\left(R^{2}\right)$ was significant, $F(10,277)=34.60, p<.000, R^{2}=.555$. Thus, this regression equation predicted $55.5 \%$ variances of the TOWRE scores. The tests of regression coefficients revealed that eight DVs predicted the TOWRE scores well. The four best predictors were Age, Handedness, PLDT-Nonword-Accuracy, and Rhyming-Speed, followed by Crossed-FLT, PLDT-Accuracy, Gender, and Rhyming-Accuracy. The Uncrossed-FLT and PLDT-Speed turned out not to be good predictors. The data are summarized in Table 13.2. 
Table 13.2

Results of Tests of Regression Coefficients

\begin{tabular}{ll}
\hline \multicolumn{1}{c}{ Dependent Variables } & \multicolumn{1}{c}{ Significance } \\
\hline Age & $p=.000$ \\
Handedness & $p=.000$ \\
PLDT-Nonword-Accuracy & $p=.000$ \\
Rhyming-Speed & $p=.000$ \\
Crossed-FLT & $P=.009$ \\
PLDT-Accuracy & $p=.015$ \\
Gender & $p=.022$ \\
Rhyming-Accuracy & $p=.031$ \\
Uncrossed-FLT & $p=.104$ \\
PLDT-Speed & $p=.527$ \\
\hline
\end{tabular}

Note: PLDT = primed lexical decision task; FLT = finger localization task .

As expected, the PLDT-Nonword-Accuracy and Age were highly correlated with the TOWRE scores (Predictions 3 \& 5). Prediction 2 was partially supported by the high correlation of Rhyming-Speed and Rhyming-Accuracy because the Condition and VF did not have any impact on the correlations. The Crossed-FLT was also significantly correlated with the TOWRE scores, whereas the Uncrossed-FLT was not (Prediction 1). Overall, accuracy appears to be a better predictor than RT since the three DVs related to accuracy predicted the TOWRE scores well; and only Rhyming-Speed was highly correlated with the TOWRE scores (Prediction 4). The high correlation of PLDTAccuracy with the TOWRE scores indicates that good lexical knowledge is a good predictor for reading. Surprisingly, Handedness was highly correlated with the TOWRE scores.

Among the significant correlations, three variables, PLDT-Nonword-Accuracy, Rhyming-Speed and Rhyming-Accuracy, are related to phonological skills. As both the dyslexia literature (e.g., Rack et al., 1992; Shaywitz et al., 2000; Snowling, 1995; 2000) and normal reading literature (e.g., Adams, 1991; Baron, 1979; Doctor \& Coltheart, 
1980; Ehri \& Wilce, 1979; Stanovich, 1982; Stanovich et al., 1984) have suggested, phonological skills are good predictors for the TOWRE scores. This finding supports the phonological deficit theory proposing that DD is primarily caused by poor phonological skills. More importantly, Crossed-FLT was significantly correlated with the TOWRE scores. This indicates that interhemispheric information transfer is associated with reading ability. This finding is compatible with previous studies (Fletcher, et a., 1982; Lindgren, 1978; Moore et al., 1996, O'Donnell, 1983; Zung, 1986) and supports the interhemispheric disconnection theory.

In summary, the DVs related to phonological skills and accuracy, Crossed-FLT, Age and Handedness were the better predictors of the TOWRE scores among the DVs of three Experiments. 


\section{Chapter 14: General Discussion}

The principal aim of the present thesis was to investigate two hypotheses: (1) DD is associated with inefficient interhemispheric communication; and (2) DD is a specific reading disorder. The first hypothesis involved investigating the interhemispheric disconnection theory. The second hypothesis was motivated by controversy in the dyslexia literature (Stanovich, 19941; Swan \& Goswami, 1997). These hypotheses were pursued using tactile, phonological and semantic tasks that may be associated with specific callosal regions.

Experiment 1 (finger localization task) investigated the involvement of inefficient interhemispheric communication in DD using a tactile task associated with callosal information transfer through the posterior midbody. Experiment 2 (rhyming judgment task) investigated the involvement of inefficient interhemispheric communication in DD using a phonological task associated with callosal information processing through the genu. Experiment 3 (primed lexical decision task) also investigated the involvement of inefficient interhemispheric communication in DD using a semantic task associated with callosal information processing through the isthmus. The first hypothesis predicted that the DD group would show less accurate and slower performance when interhemispheric communication was required in information processing (i.e., Crossed or Bilateral Conditions) than when it was not (i.e., Uncrossed or Unilateral Conditions). The second hypothesis predicted that the DD group would show different patterns of task performance from the CAP group. 
Ellis (1994) argued that reading, unlike speech, is not lateralized in the brain, and thus, reading requires multiple brain systems in both hemispheres, including visual, spatial, phonological and semantic systems, memory, and attention. As discussed in Chapter 1, the primal disorder of DD is reading, particularly, poor phonological processing (e.g., Rack et al., 1992; Snowling 2000). However, this reading disorder is expressed in diverse ways, including semantic processing (Castles \& Coltheart, 1993; Temples, 1994), sequential processing (Bakker, 1973), Crossed-finger localization (Fabbro, et al, 2001; Gross-Glenn et al., 1984; Summerfield \& Michie, 1993), bimanual motor coordination (Gladstone et al., 1989; Wolff et al., 1990), memory (Macaruso et al., 1995; Swan \& Goswami, 1997), and attention (Hynd et al., 1990). Considering the involvement of various brain systems in reading processing and the diverse deficits demonstrated by DD, it is essential to investigate multiple brain systems in order to understand DD. However, most theories of DD have investigated only a single deficit of $\mathrm{DD}$, for example, phonological processing in the phonological deficit theory (e.g., Bryant, \& Goswami, 1987, Ellis, 1991; Goswami \& Bryant, 1990; Gough \& Tunmer, 1986; Kats, 1986; Lundberg, 1989; Snowling, 1981; Snowling, 1995; Snowling \& Hulme, 1994; Vellutino, 1979), the magnocellular visual system in the magnocellular deficit theory (Eden, VanMeter, Rumsey et al., 1996; GrossGlenn, Skottun, Glenn et al., 1995; Livingstone, Rosen, Drislane, \& Galaburda, 1991; Stein \& Walsh, 1997; Talcott, Hansen, Willis-Owen et al., 1998), or language areas in the left hemisphere dysfunction theory (e.g., Drake, 1968; Galaburda \& Kemper, 1979; Galaburda et al., 1985; Pugh et al., 2000; Shaywitz et al., 2002). As a result, these theories provided evidence that can explain one of the characteristics of DD associated with a specific deficit, 
but tend to leave the other characteristics unexplained. In contrast, the interhemispheric disconnection theory has provided evidence that can accommodate different characteristics of DD, for example, poor bimanual motor coordination (Gladstone et al., 1989; Wolff et al., 1990), and poor Crossed-finger localization (Fabbro, et al, 2001; Gross-Glenn et al., 1984; Summerfield \& Michie, 1993), as well as poor phonological processing associated with children with callosal agenesis (Temple \& Ilsley, 1994; Temple et al., 1989).

The challenge of the present thesis was to focus on three cerebral functions associated with the callosal regions and to attempt to understand DD associated with inefficient interhemispheric communication by examining a combination of these functions. The findings could thus demonstrate the involvement of inefficient interhemispheric communication in DD and provide evidence to account for the different characteristics of DD. By the same token, the findings could dissociate DD from poor reading disability based on a single characteristic or a combination of characteristics of DD.

The finger localization task was important in establishing the foundation for the present thesis by replicating the previously observed pattern of inefficient interhemispheric communication with respect to the processing of tactile sequential information in DD children. The significant findings in Experiment 1 were: (1) finger localization was more accurate in the Uncrossed than the Crossed Condition across all the groups; and (2) the DD and YA groups demonstrated lower scores than the CA group in both the Crossed and Uncrossed Conditions. These findings were consistent with Summerfield and Michie's study. However, the DD group did not show significantly lower scores than the YA group as the DD children in Summerfield and Michie's study did. This could be accounted for by 
sampling differences between the two studies, i.e., Summerfield and Michie's study tested younger DD children $(M$ age $=9.0$ years, myelination has not completed yet $)$ and compared them to their reading age-matched group ( $M$ reading $=7.0$ years). The DD students in the present study were older $(M$ age $=13.08$ years $)$ and were enrolled in specialized school programs (i.e., intensive and best interventions). As discussed in Chapter 10, the DD children who participated in the present thesis experiments have received excellent training programs for many years, and thus, it is possible that this good training compensated for inefficient interhemispheric communication, while, the young DD children in Summerfield and Michie's study may not have received enough training to compensate for inefficient interhemispheric communication due to young age of their participants, i.e., it is assumed that that young DD children have less opportunities to have training than old DD children. More importantly, the regression analysis revealed that accuracy in the finger localization in the Crossed condition was positively correlated with the TOWRE scores, suggesting that the finger localization task performance in the Crossed condition is associated with reading ability. This is also consistent with Summerfield and Michie's study and supports the interhemispheric disconnection theory. Overall, Experiment 1 replicated the main significant findings of their study, suggesting that the foundation for the present thesis was established.

There were several common findings throughout the three experiments. First, as expected, the DD group demonstrated significantly slower and less accurate performance than the CA group in tactile, phonological and semantic information processing across the different task demands. This implies that all three tasks of the present thesis were related to 
reading ability. This implication is compatible with previous studies suggesting that phonological skills predict reading (Adam, 1991; Baron, 1979; Doctor \& Coltheart, 1980; Ehri \& Wilce, 1979; Stanovich, 1983; Stanovich et al., 1984); and Crossed-finger localization is correlated with reading test scores (Fletcher et al., 1982; Lindgren, 1978; Moore et al., 1996; O'Donnell, 1983; Zung, 1986

Second, the DD and YA groups demonstrated similar patterns of performance in speed and accuracy across the different task demands. It appears that the developmental delay model can account for DD (Gough \& Turner, 1983). According to Frith's stage model (1985) of reading acquisition, children normally pass through the developmental reading stages in order to master reading skills before they become fluent readers. Geffen et al. (1985) provided evidence that callosal efficiency normally improves with age and development of callosal maturity. Thus, the developmental delay model assumes that DD children may take more time to go through these developmental stages of reading acquisition and the developmental processes of callosal efficiency than normal children do; however, the model asserts that eventually DD children can catch up with normal children. Nevertheless, this model cannot provide a full explanation as to why the DD group ( $M$ age $=13.04 \mathrm{y}$ ears, $M$ reading age $=\mathbf{8 . 0 2}$ years), who were much older and less skilled in reading than the YA group $(M$ age $=9.01$ years, $M$ reading age $=12.06$ years $)$, demonstrated similar patterns of performance to the YA group.

Third, the CAP group did not significantly differ in speed and accuracy from the DD, YA and CA groups across the different task demands. Because the CAP group had a similar reading age $(M$ reading age $=11.0$ years) to the YA group; and this group (IQ $=$ 
83.8) had lower non-verbal IQ scores than the DD group (IQ = 102.7), YA group (IQ = 105.0) and CA group (IQ = 102.7), the CAP group's similar performance in speed and accuracy to the other groups suggests that the three tasks are associated with both reading ability and age-related components independent of IQ scores.

Fourth, the standardized reading (TOWRE) scores correlated with the task scores of the three experiments in both the Crossed (Bilateral) and the Uncrossed (Unilateral) Conditions. This implies that reading is associated with both intrahemispheric information processing and interhemispheric communication. This implication accords with the Ellis's view (1994) that reading requires multiple brain functions in the brain.

Fifth, the RVF superiority effect was observed in the rhyming judgment task and the primed LDT. This validated the present visual half-field procedures. The presence of this effect suggests that the LH of the DD group primarily processed the experimental stimuli. This does not support the left hemisphere dysfunction theory.

There were two critical findings for the present thesis. The first was a three-way interaction of Group $\mathrm{x}$ Visual $\mathrm{x}$ Condition in Experiment 2. This interaction revealed that the CAP group responded significantly faster in the Bilateral RVF condition than the Unilateral RVF condition. A series of Banich studies (Banich \& Belger, 1990; Belger \& Banich, 1992) suggested that using both hemispheres enhances efficiency in information processing for complex tasks (see Chapters $11 \& 12$ ). Since a rhyming judgment task is a complex task, the faster responses in the Bilateral than the Unilateral RVF condition demonstrated by the CAP group was possibly due to more efficient information processing in bilateral presentationss. Since accuracy in both conditions was similar in the CAP group, 
but RT was faster in the Crossed condition, the latter finding does not appear to reflect a speed-accuracy trade-off.

Furthermore, the three-way interaction revealed that the YA group responded significantly less accurately to the Bilateral RVF condition compared to the Unilateral RVF condition. This suggests that the bilateral presentations did not enhance efficiency of information processing for the YA group. Previous developmental studies (Galin et al., 1977; Quinn \& Geffen, 1986) suggest that efficiency of the CC improves with age. Considering that the students in the YA group were good readers and had high non-verbal IQ scores, the inefficient information processing in bilateral presentations demonstrated by the YA group was possibly due to inefficient callosal information processing. It must be noted that since an RVF superiority effect was present in this task, it was assumed that the LH was primarily responsible for this phonological task. Thus, the significant difference in the RVF between the Unilateral and Bilateral Conditions was particularly important for Experiment 2.

More importantly, the DD group failed to demonstrate the more efficient information processing in bilateral presentations. Furthermore, their response patterns in the Bilateral RVF condition resembled the YA group's, i.e., they responded in the Bilateral RVF condition more slowly than in the Unilateral RVF condition. This difference within the DD group was a trend. However, the difference between the DD group $(M \Delta=+62.56$ msec) and CAP group ( $M \Delta=-146.45 \mathrm{msec})$ of the two RVF conditions was significant. It showed a quantitatively different, i.e., a positive value for the DD group and a negative value for the CAP group. This finding strongly suggests that the DD group was less 
inefficient information processing in bilateral presentations, as did the YA group. This finding supports Hypothesis 1, that DD is associated with inefficient interhemispheric communication. Since the pattern of performance was different between the DD and CAP groups, this finding also supports Hypothesis 2, that DD is a specific reading disorder. This also supports the phonological deficit theory because the DD children performed more poorly than the CA group in this phonological task. The finding fits well with this theory that claims that DD is a linguistic disorder and manifests itself specifically in phonological processing (e.g., Rack et al., 1992; Snowling 1995, 2000).

Although the finding supports the main two hypotheses, a question remains as to why the DD group did not demonstrate a significant difference between the Bilateral RVF and the Unilateral RVF condition as the YA group did. It is possible to assume that the students' intensive training might affect strategies in reading.

The second critical finding was the priming effects demonstrated by the DD group in Experiment 3. The DD and CA groups demonstrated faster and more accurate responses in the associated pairs than the non-associated pairs. This indicates that the DD and CA groups obtained priming effects. Since priming effects represent the strength of lexical relatedness in the mental lexicon (Anderson, 1976; 1983; Collins \& Quillian, 1969; Collins \& Loftus, 1975; Neely, 1977, 1991), the significant priming effects in the DD group indicate that the DD children have good semantic connections in the mental lexicon. This finding is consonant with previous studies (Kochnower et al., 1983; Siegel \& Ryan, 1988; Swan \& Goswami, 1997). In terms of the CAP group, their priming effects in the accuracy data might have resulted from a speed-accuracy trade-off, because this group did not show 
a priming effect in RT. This suggests that the CAP group might not have good semantic connections in the mental lexicon. Thus, a lack of semantic connections in the mental lexicon might contribute to the CAP group's reading problems, whereas this would not apply to the DD group, since this group had good semantic connections in the mental lexicon. This finding supports Hypothesis 2 that DD differs qualitatively from general poor reading ability.

Nonword processing is critical for the investigation of DD because ample dyslexia research (e.g., Olson et al., 1985; Rack et al., 1994; Snowling, 1981; Snowling, 1995, 2000) has suggested that poor phonological processing is the major deficit of $\mathrm{DD}$, and it is typically reflected in poor nonword reading. The finding of Experiment 3 added evidence of poor phonological processing of DD, i.e., the DD group responded significantly more slowly and less accurately than the CA group. Interestingly, however, the responses of the DD group did not significantly differ from those of the CAP group in both RT and accuracy. This suggests that the CAP group also had similar phonological problems to the DD group. In a word, poor phonological processing might be a common characteristic of the DD and CAP groups. This finding also supports the view (Stanovich, 1994; Siegel \& Stanovich, 1994) that poor readers with high IQs (i.e., DD) and low IQs (i.e., CAP) do not qualitatively differ.

In Experiment 3, unexpectedly, the Condition (Bilateral, Unilateral) variable did not have any effect on either dependent variable (i.e., RT and accuracy). This finding was consistent with that in Clarke and Zaidel's study (1994) with normal university students. It 
suggests that the DD group processed the prime in a similar manner to the other groups. This does not support the interhemispheric disconnection theory.

The regression analysis revealed that the DVs related to phonological skills were good predictors of the TOWRE scores. This is consistent with the idea that phonological skills are good predictors of reading as suggested in the reading literature (Adam, 1991; Baron, 1979; Doctor \& Coltheart, 1980; Ehri \& Wilce, 1979; Stanovich, 1983; Stanovich et al., 1984). It also revealed that the accuracy data were a better predictor than the RT data. As expected, Age was highly correlated with the TOWRE scores. The task scores in each experiment also showed positive correlations with Age. These correlations suggest that agerelated components are associated with tactile, phonological and semantic processing as well as reading ability.

As discussed earlier, the challenge of this thesis was to attempt to understand DD by investigating the inefficient interhemispheric communication in different domains. From that perspective, new pictures of DD have emerged from the present findings. The first picture is that the involvement of interhemispheric communication in DD may vary depending on type of information, because in Experiment 2, less efficient phonological information processing in bilateral presentations was observed in the DD group. However, this inefficient information processing was not observed in the semantic task in Experiment 3. These findings support the interhemispheric disconnection theory if the putative inefficient interhemispheric communication only affects anterior regions. They also support the phonological deficit theory because inefficient information processing was observed for the DD group in phonological processing. In the finger localization task, it was not clear 
why there was no group difference in the CUD (Crossed-Uncrossed-Difference). Because the CUD generally reflects the difference between the Crossed and the Uncrossed conditions, it is possible to assume that the present negative results observed in the DD group might be a floor effect in the Uncrossed condition, i.e., affected by a significant difference between these the DD group and the CA group in the Unilateral condition. Commissurotomized patients (Geffen et al., 1985) and children with callosal agenesis (Lassonde et al., 1988) showed significantly lower scores than the control groups in the Uncrossed in the 2-Finger sequence but not in the single finger localization. This suggests that the $\mathrm{CC}$ might be essential in sequence processing in intrahemispheric communication. If this is the case, the poor performance of the DD group to the CA group in the Unilateral condition could be due to inefficient callosal information processing, and the negative CUD reflected the floor effect in the Uncrossed condition.

If inefficient interhemispheric communication is reflected in poor performance in the Crossed condition, this would not be distinct to the DD group but common in the DD and the CAP groups. The theory is compatible with a finding by Moor et al. (1996) that a finger localization in the Crossed condition was related to a rhyming judgment ability in general, i.e., children who had poor phonological skills tend to show poor performance in a finger localization task in the Crossed condition. Because Summerfield and Michie's (1993) study did not include a CAP group, more research is needed to investigate this possibility.

The second picture of DD is that RT and accuracy alone cannot define DD. This is because the DD and CAP groups demonstrated similar patterns of performance across the experimental tasks for both measures, including nonword reading in Experiment 3. Ample 
studies (Adam, 1991; Baron, 1979; Doctor \& Coltheart, 1980; Ehri \& Wilce, 1979;

Stanovich, 1983; Stanovich et al., 1984) have suggested that poor phonological skills of DD children are typically reflected in nonword reading. Thus, poor nonword reading is considered to be one of the main characteristics of DD. However, the present finding suggests that poor nonword reading is not distinct to DD but common in both DD and poor reading ability. This supports Stanovich's (1991) view that children with high and low IQ do not differ in nonword reading. Differences in the two groups emerged in bilateral presentations in the phonological task and priming effects in the semantic task. This is a good example demonstrating the strength of the research approach of the present thesis, which took multiple characteristics of DD into account into its investigation of DD.

The third picture is that when the results of Experiment 2 and 3 were integrated, it clearly demonstrates that the main problems of DD children lie in phonological processing but not in semantic processing. This supports the phonological deficit theory. It also suggests that the developmental delay theory cannot account for $\mathrm{DD}$. This is because the DD group demonstrated intact semantic connections in the mental lexicon but the YA group did not, suggesting that they are qualitatively different.

There are some limitations of the present thesis. First, there was no RA control group in the present thesis because of difficulties in recruiting students (see Chapter 9). The inclusion of an RA group in the investigation of DD can be considered important. Swan and Goswami (1997) reasoned that since general cognitive development in DD children could be delayed due to reading problems, it is not clear whether the fact that DD children performed more poorly than their CA controls in their experimental task stems from 
reading ability or slow cognitive development. When the DD group demonstrated significant difference from the CA group and performed more poorly than the RA group, it suggests that the difference is associated with DD. In the present thesis, the DD group ( $M$ age $=13.08$ year, $M$ reading age $=8.02$ years $)$ was compared to the $Y A$ group $(M$ age $=$ 9.01 years, $M$ reading age $=12.06$ year), who had far better reading ability for chronological age. Second, in Experiment 1, the performance in the DD, CAP, and YA groups was very poor in the 4-Finger sequence in the Crossed condition. This reflected that the tasks were hard for most of the participants. In future experiments, a finger localization task should be carried out with easier tasks. Third, the group size ratio between the CA group $(\mathrm{n}=25)$ and the CAP group $(\mathrm{n}=10)$ might have affected statistical power in the present thesis. Tabachnick and Fidell (1996) suggest that unequal group sizes typically provide no special difficulty in repeated measures analysis because each hypothesis is tested as if in a one-way design. However, Stevens (1996) also suggests that group sizes would affect statistical power (the probability of rejecting a null hypothesis when it is false): if group sizes are approximately equal (largest/smallest $=1.5$ ), $F$ is robust, but when group sizes are sharply unequal and if large sample variances are associated with small group size, the $F$ statistic is liberal. A statistic's being liberal means that we falsely reject the null hypothesis when it is true greater than $\alpha$ level of significance frequently (Stevens, 1996). In the present study, the larger variances were often associated with the CAP group in the three experiments. This suggests that the actual $\alpha$ level of significance in the present thesis might be more than $\alpha(.05)$ level of significance. Fourth, In Experiment 3, the small effect size $\left(\eta^{2}=.012\right)$ might have affected statistical power to detect significant group 
differences related to the Condition variable. Because statistical power depends on both sample sizes and effect sizes, it is important to get as large an effect size as possible to increase statistical power.

In conclusion, the present findings provide the following evidence in support of Hypothesis 1, that DD is associated with inefficient interhemispheric communication (the interhemispheric disconnection theory): (1) the DD group showed a significant difference from the CA group in the Crossed condition, which was also observed in the YA and CAP groups; (2) the finger localization scores in the Crossed condition were found to be a good predictor of the reading (TOWRE) scores; and (3) the DD group demonstrated a disadvantage of bilateral presentations in the phonological task. In terms of Hypothesis 2, that DD is a specific reading disability, the present findings strongly support this hypothesis with the following evidence: (1) the DD group demonstrated inefficient information processing in bilateral presentations, whereas the CAP group demonstrated efficient information processing in bilateral presentations in the phonological task, suggesting that the CAP children demonstrated efficient callosal connectivity but the DD group did not; and (2) the DD group demonstrated priming effects, whereas the CAP did not, suggesting that the DD children had intact semantic connections in the mental lexicon, but the CAP children did not.

For future research, task difficulty in the finger localization task needs to be controlled in order to avoid a floor effect, such as blocking the finger sequence in each condition to be able to measure performance in the Crossed Condition. If each condition has 2-Finger Sequence trials followed by 3-Finger and 4-Finger sequence trials, the task 
becomes easier than when using a sequence in which 2-Finger, 3-Finger and 4-Finger sequences are scrambled. Since most previous dyslexia studies, including Summerfield and Michie's study (1993), did not include a CAP group, the finding of the present study, i.e., that inefficient interhemispheric communication in the posterior midbody are associated with general reading ability, needs to be replicated with equal group sizes.

A primed LDT also needs to be performed with a short ISI to increase the effect size. In a short ISI (i.e., around $250 \mathrm{msec}$ ), the task demands increase in terms of information processing speed (i.e., fluency) for poor readers but not for good readers. Thus, a short ISI may possibly dissociate DD children from control groups, since DD children are poor in fluency (Denkla, 1975; Denkla \& Rudel, 1976). It is also necessary to increase the effect size to see if the absence of effect on the Condition variable in the present study was truly due to a small effect size or to a lack of efficient interhemispheric communication.

Finally, since the DD group demonstrated good semantic connections in the mental lexicon, their reading problems might stem from the process of retrieving lexical knowledge from long term memory as previous studies have suggested (Swan \& Goswami, 1997; Macaruso et al., 1995). In particular, working memory (WM) is essential for the retrieval process in reading while searching appropriate sounds for a word in the mental lexicon (Adam \& Gathercole, 1996; Baddeley, 1981; Breznitz, 1997; Gathercole \& Baddeley, 1990; Jorm, 1983; Mann \& Liberman, 1984; Muter et al., 1998). Including memory components in the investigation of DD will give us further insights in understanding DD. 


\section{References}

Aboitiz, F., Scheibel, A. B., \& Zaidel, E. (1992). Morphometry of the Sylvian fissure and the corpus callosum, with emphasis on sex differences. Brain, 115, 15211541.

Aboitiz, F., Scheibel, A. B., Fisher, R. S., \& Zaidel, E. (1992). Fiber composition of the human corpus callosum. Brain Research, 598, 143-153.

Adams, A. \& Gathercole, S. E. (1996). Phonological working memory and spoken language development in young children. The Quarterly Journal of Experimental Psychology, 49A(1), 216-233.

Adams, M. J. (1990). Analyzing the reading process: Orthographic processing. In Adam, M. J., Beginning to read (Chap. 6), (pp.107-135). Cambridge, Massachusetts, London, England: The MIT Press.

Aglioti, S., Beltramello, A., Tassinari, G., \& Berlucchi, G. (1998). Paradoxically greater interhemispheric transfer deficits in partial than complete callosal agenesis. Neuropsychologia, 36, 1015-1024.

Akelaitis, A. J., Risteen, W. A., Wagenen, W. P. (1942). Studies on the corpus callosum. III. A contribution to the study of dyspraxia in epileptics following partial and complete section of the corpus callosum. Archives of Neurology and Psychiatry, 47, 971-1008.

Anastasi, A., \& Urbina, S. (1997). Psychological testing ( $7^{\text {th }}$ ed.). Upper Saddle, River, NJ: Prentice-Hall.

Anderson, J. R. (1976). Language, memory, and thought. Hillsdale, N. J.: Lawrence Erlbaum Associates.

Annett, M. (1967). The binomial distribution of right, mixed and left handedness. Quarterly Journal of Experimental Psychology, 19, 327-333.

Backes, W., Vuurman, E., Wennekers, R., Spronk, P., Wuisman, M., van Engelshoven, J., \& Jolles, J. (2002). Atypical brain activation of reading processes in children with developmental dyslexia. Journal of Child Neurology, 17, (12), 867-871. 
Baddeley, A. (1981). The concept of working memory: A view of its current state and probable future development. Cognition, 10, 17-23.

Baddeley, A. (1992). Science, 255, 556-559.

Baddeley, A. D. \& Hitch, G. (1974). Commentary on 'Working memory'. In Bower, G. (Eds.), Human Memory Basic Processes. New York: Academic Press.

Baddeley, A. D. (1986). Working Memory. Oxford: Clarendon Press.

Baddeley, A. D., Ellis, N. C., Miles, T. R., \& Lewis, V. J. (1982). Developmental and acquired dyslexia: a comparison. Cognition, 11, 185-199.

Badian, N. A., \& Wolff, P. H. (1977). Manual asymmetries of motor sequencing in reading disability. Cortex, 13, 343-349.

Bakker, M. G. (1973). Ear dominance and reading ability. Cortex, 9, 310-312.

Bakker, M. G. (1979). Perceptual asymmetries and reading proficiency. In Bortner, M. (Eds.), Cognitive growth and development, pp.134-152. New York: Brunner Mazel.

Bakker, M. G. (1992). Neuropsychological classification and treatment of dyslexia. Journal of Learning Disabilities, 25, 102-109.

Banich, M. T. (1997). Hemispheric Specialization. In Banich, M. T. (Eds.), Neuropsychology: The neural bases of mental function (pp.110-111). Boston: Houghton Mifflin Company.

Banich, M. T. \& Belger, A. (1990). Interhemispheric interaction: How do the hemispheres divide and conquer a task? Cortex, 26, 77-94.

Banich, M. T. \& Karol, D. L. (1992). The sum of the parts does not equal the whole: Evidence from bihemispheric processing. Journal of Experimental Psychology: Human Perception and Performance, 18 (3), 763-784.

Banich, M. T., Passarotti, A. M., \& Janes, D. (2000). Interhemispheric interaction during childhood: I. Neurologically intact children. Developmental neuropsychology, 18(1), 33-51.

Baron, J. (1979). Orthographic and word-specific mechanisms in children's reading of words. Child Development, 50, 386-393. 
Baynes, K., Tramo, M. J., \& Gazzaniga, M. S. (1992).Reading with a limited lexicon in the right hemisphere of a callosotomy patient. Neuropsychologia, 30(2), 187-200.

Baynes, K., Wessinger, C. M., \& Gazzaniga, M. S. (1994). Lexical decision with unlimited exposure duration in the right and left hemispheres of a commissurotomy patient. Poster, Cognitive Neuroscience Society, San Francisco, CA.

Beaton, A. (1985). Left side, Right side: A review of laterality research. London: Batsford.

Beech \& Harding (1984). Phonemic processing and the poor reader from the developmental point of view. Reading Research Quarterly, 19, 357-366.

Benson, D. F, \& Geschwind, N. (1985). The aphasias and related disturbances. In Heilman, K. M. \& Valestein, E. (Eds.), Clinical Neuropsychology ( ${ }^{\text {rd }}$ ed.) (pp.1736), New York: Oxford University Press.

Best, M. \& Demb, J. B. (1999). Normal planum temporale asymmetry in dyslexics with a magnocellular pathway deficit. Brain Imaging, 10, 607-612.

Black, P., \& Myers, R. E. (1964). Visual function of the forebrain commissures in the chimpanzee. Science, 146, 799-800.

Boder, E. (1971). Developmental dyslexia: Prevailing diagnostic concepts and a new diagnostic approach. In Mykleburst, H. R. (Eds.) Progress in learning disabilities, $I I$ (pp. 293-321). New York: Grune \& Stration.

Boder, E. (1973). Developmental dyslexia: A diagnostic approach based on three atypical reading-spelling patterns. Developmental Medicine and Child Neurology, $15,663-687$.

Bogen, J. E. \& Gazzaniga, M. S. (1965). Cerebral commissurotomy in man. Minor hemisphere dominance for certain visuospatial functions. Journal of Neurosurgery, 23, 394-399.

Bogen, J. E., Vogel, P. J. (1962). Cerebral commissurotomy in man: Preliminary case report. Bulletin of the Los Angeles Neurological Societies, 27, 191-220. 
Borsting, E., Ridder, W. H., Dudeck, K., Kelly, C., Matsui, L., \& Motoyama, J. (1996). The presence of a magnocellular deficit depends on the type of dyslexia. Vision Research, 36, 1047-1053.

Bradley, L. B. \& Bryant, P. E. (1978). Difficulties in auditory organisation as a possible cause of reading backwardness. Nature, 271, 746-747.

Bradley, L. B. \& Bryant, P. E. (1983). Categorizing sounds and learning to read-a causal connection. Nature, 301 February, 399-421.

Bradshaw, J. \& Nettleton, N. (1981). The nature of hemispheric specialization in man. Behavioural Brain and Science, 4, 51-91.

Breznitz, Z. (1997). Effects of accelerated reading rate on memory for text among dyslexic readers. Journal of Educational Psychology, 89(2), 289-297.

Bridgman, C. S., \& Smith, K. U. (1940). Bilateral neural integration in visual perception after section of the corpus callosum. Journal of Comparative Neurology, 83, 57-68.

Broca, P. (1865). Sur le siège de la faculté du langage articulé (On the site of he faculty of articulate speech). Bulletins de la Société d'Anthropologie, Paris, 6, 377-393.

Brown, L., Sherbenou, R., Johnson, S. K. (1980). Test of Non-Verbal Intelligence-3. Illinois: Stoelting Co.

Bruck, M. (1988). The word recognition and spelling of dyslexic children. Reading Research Quarterly, 23, 51-69.

Brunswick, N., McCrory, E., Price, C. J., Firth, C. D., \& Frith, U. (1999). Explicit and implicit processing of words and pseudowords by adult developmental dyslexics A search for Wernicke's Wortschata? Brain, 122, 1901-1917.

Bryant, P. \& Goswami, U. (1987). Phonological awareness and learning to read. In Beec, J. R. \& Colley, A.M. (Eds.), Cognitive Approaches to Reading (pp. 214243). New York: John Wiley \& Sons Ltd.

Bryant, P. (1998). Sensitivity to onset and rhyme does predict young children's reading: A comment on Muter, Hulme, Snowling, and Taylor (1997). Journal of Experimental Child Psychology, 71, 29-37. 
Bryant, P., \& Impey, L. (1986). The similarities between normal readers and developmental and acquired dyslexics. Cognition, 24, 121-137.

Bryden, M. P. (1982). Laterality: Functional asymmetry in the intact brain. NY \& London: Academic Press.

Brysbaert, M \& d'Ydewalle, G. (1990). The difference between stimulus and background: An explanation of the psychophysical function based on partition scales? In G. Hans-Georg (Ed.), Psychophysical explorations of mental structures (pp. 58-70). Kirkland, WA: Hogrefe \& Huber Publishers.

Burgess, C. \& Simpson, G. B, (1988). Cerebral hemispheric mechanisms in the retrieval of ambiguous word meanings. Brain And Language, 33, 86-103.

Burr, D. C., Morrone, M. C., \& Ross, J. (1994). Selective suppression of the magnocellular visual pathway during saccadic eye movements. Nature, 371, 511513.

Burton, M. W., Small, S. L., \& Blumstein, S. E. (2000). The role of segmentation in phonological processing: An $\mathrm{AMRI}$ investigation. Journal of Cognitive Neuroscience, 12 (4), 679-690.

Byne, W., Bleier, R., \& Houston, L. (1988). Variations in human corpus callosum do not predict gender: A study using magnetic resonance imaging. Behavioral Neuroscience, 102, 705-712.

Castles, A., \& Coltheart, M. (1993). Varieties of developmental dyslexia. Cognition, 47, 121-131.

Chiarello, C. A. (1980). A house divided? Cognitive functioning with callosal agenesis. Brain and Language, 11, 128-158.

Chiarello, C. (1985). Hemisphere dynamics in lexical access: automatic and controlled priming. Brain And Language, 26, 1460172.

Chiarello, C. A. (1995). Does the corpus callosum play a role in the activation and suppression of ambiguous word meaning? In Kitterle, F. L. (Eds.), Hemispheric communication: Mechanisms and models. (pp. 177-188). Hillsdale, NJ: Lawrence Erlbaum Associates. 
Chiarello, C. \& Beeman, M. (1998). Right hemisphere language comprehension. Perspectives from cognitive neuroscience. Mahwah, NJ: Laurence Erlbaum Associates.

Chiarello, C., Burgess, C., Richards, L., \& Pollock, A. (1990). Semantic and associative priming in the cerebral hemispheres: some words do, some words don't...sometimes, some places. Brain and Language, 38, 75-104.

Chiarello, C. \& Richards, L. (1992). Another look at categorical priming in the cerebral hemispheres. Neuropsychologia, 30, 381-392.

Clarke, S. \& Zaidel, E. (1994). Anatomical-behavioral relationships: corpus callosum morphometry and hemispheric specialization. Behavioural Brain Research, 64, 185-202.

Clarke, S. Kraftsik, R., Van Der Loos, H., Innocenti, G. M. (1989). Forms and measures in adult and developing human corpus callosum: Is there sexual dimorphism? Journal of Comparative Neurology, 280, 213-230.

Collins, A. M., \& Loftus, E. F. (1975). A spreading activation theory of semantic processing. Psychological Review, 82, 407-428.

Collins, A. M., \& Quillian, M.. R. (1969). Retrieval time from semantic memory. Journal of Verbal Learning and Verbal Behavior, 8, 240-247.

Coltheart, M. (1980). Deep dyslexia: a review of the syndrome. In Deep Dyslexia, M. Coltheart, K. E. Patterson, \& J. Marshall (eds.). London: Routledge and Kegan Paul.

Coltheart, M. (1983). The right hemisphere and disorders of reading. In Functions of the Right Cerebral Hemisphere, A. Young (eds.). London: Academic Press.

Coltheart, M., Curtis, B., Atkins, P., \& Haller, M. (1993). Models of reading aloud: Dual route and parallel-distributed-processing approaches. Psychological Review, 100, 589-608.

Coltheart, M., Davelaar, E., Jonasson, J. T., \& Besner, D. (1977). Access to the internal lexicon. In Dornic, S. (Eds.), Attention and performance VI (pp. 64-85). Hillsdale NJ: Erlbaum. 
Coltheart, M. \& Leahy, J. (1996). Assessment of lexical and nonlexical reading abilities in children: some normative data. Australian Journal of Psychology, 48(3), 136140 .

Coltheart, M., Masterson, J., Byng, S., Prior, M., \& Riddoch, J. (1983). Surface dyslexia. Quarterly Journal of Experiment Psychology, 37A, 469-495.

Cook, N. D. (1986). The brain Code. (pp.52-75). New York: Methuen \& Co. Ltd. Corballis, M. C. (1974). The left-right problem in psychology, Canadian Psychologist, 15.

Corballis, M. C., \& Finlay, D. C. (2000). Interhemispheric visual integration in three cases of familial callosal agenesis. Neuropsychology, 14, 60-70.

Corina, D. P., Richards, T. L., Serafini, S., Richards, A. L., Steury, K., Abbott, R. D., Echelard, D. R., Maravilla, K. R., Berninger, V. W. (2001). fMRI auditory language differences between dyslexic and able reading children NeuroReport, 12(6), 1195-1201.

Corkin, S. (1974). Serial-ordering deficits in inferior readers. Neuropsychologia, 12, 347-354.

Cornelissen, P., Richardson, A., Mason, A., Fowler, S., \& Stein, J. (1995). Contrast sensitivity and coherent motion detection measured at photopic luminance levels in dyslexics and controls. Vision Research, 35, 1483-1494.

Cornish, K. M. (1996). The Geschwind and Galaburda theory of cerebral lateralisation: An empirical evaluation of its assumptions. Current Psychology: Developmental, Learning, Personality, and Social Spring, 15, 68-76.

Cowan, N. (1995). Attention and Memory (pp. 81). Oxford: Oxford University Press.

Critchley, M. (1970). Specific developmental dyslexia. In Lenneberg, E. H. \& Lenneberg, E. (Eds.), Foundations of language development, 2. New York: Academic Press.

Crowder, R. C. (1982). The psychology or reading. New York: Cambridge University Press. 
Dalby, M. A., Elbro, C. \& Stodkilde-Jorgensen, H. (1998). Temporal lobe asymmetry and dyslexia: An in vivo study using MRI. Brain and Language, 62, 51-69.

Dandy, W. R. (1936). Operative experience in cases of pineal tumors. Archives of Surgery, 33, 19-46.

Davidson, H. D., Abraham, R., \& Steiner, R. E. (1985). Agenesis of the corpus callosum: magnetic resonance imaging, Radiology, 155, 371-373.

De Lacoste, M. C., \& Holloway, R. L. (1982). Sexual dimorphism in the human corpus callosum. Science, 216, 1431-1432.

De Lacoste, M. C., Kirkpatrick, J. B., \& Ross, E. D. (1985). Topography of the human corpus callosum Journal of Neuropathology and Experimental Neurology, 44, 578-591.

DeArmond, S. J. Fusco, M. M., \& Dewey, M. M. (1974). Structure of the human brain, A photographic atlas. New York: Oxford University Press.

DeFries, J. C., Fulker, D. W., \& LaBuda, M. C. (1987). Evidence for a genetic etiology in reading disability of twins. Nature, 329, 537-539.

DeFries, J. C., Gillis, J. J., \& Wadsworth, S. J. (1993). Genes and genders: A twin study of reading disability. In Galaburda, A. M. (Eds.), Dyslexia and Development, Neurobiological Aspects of Extra-Ordinary Brains (pp. 187-204). Cambridge, Massachusetts, and London: Harvard University Press.

Demb, J. B., Boynton, G. M., \& Heeger, D. J. (1997). Brain activity in visual cortex predicts individual differences in reading performance. Proceeding National Academy of Science, USA, 94, 13363-13366.

Demb, J.B., Boynton, G. M., Best, M., \& Heeger, D. J. (1998). Psychophysical evidence for a magnocellular pathway deficit in dyslexia. Vision Research, 38, 555-559.

Denenberg, V. H. (1981). Hemispheric laterality in animals and the effects of early experience. Behavioral Brain Science, 4, 1-21. 
Denenberg, V. H., Kertesz, A., \& Cowell, P. E. (1991). A factor analysis of the human's corpus callosum. Brain Research, 548, 126-132.

Denkla, M. (1974). Development of motor coordination in normal children. Development Medicine of Child Neurology, 16, 729-741.

Denkla, M. B. \& Rudel, R. G. (1976). Rapid 'Automatized' naming (R.A.N): Dyslexia differentiated from other learning disabilities. Neuropsychologia, 14, 471-479.

Dennis, M. (1981). Language in a congenitally acallosal brain. Brain and Language, 12, 33-53.

DeYoe, E. A. \& Van Essen, D. C. (1988). Concurrent processing streams in monkey visual cortex. Trends Neuroscience, 11, 219-226.

Doctor, E. A. \& Coltheart, M. (1980). Children's use of phonological encoding when reading for meaning. Memory and Cognition, 8, 195-209.

Doty, R. W. \& Negrao, N. (1973). Forebrain commissures and vision (pp.543-582). In Jung, R. (Eds.), Handbook of Sensory Physiology, VII, Berlin: Springer.

Drake, W. E. (1968). Clinical and pathological findings in a child with a developmental learning disability. Journal of Learning Disabilities, 1, 486-502.

Duara, R., Kushch, A. Gross-Glenn, K., Barker, W. W., Jallad, B., Pascal, S., Loewenstein, D. S., Sheldon, J., Rabin, M., Levin, B., \& Lubs, H. (1991). Neuroanatomic differences between dyslexic and normal readers on Magnetic Resonance Imaging Scans. Archives of Neurology, 48, 410-416.

Eden, G. F., VanMeter, J. W., Rumsey, J. M., Maisog, J. M., Woods, R. P., \& Zeffiro, T. A. (1996). Abnormal processing of visual motion in dyslexia revealed by functional brain imaging. Nature, 382, 66-69.

Ehri, L. C. (1991). The development of reading and spelling in children: An overview. In M. Snowling, \& M. Thomson ( $2^{\text {nd }} \mathrm{ed}$.), Dyslexia Integrating Theory \& Practice (pp. 63-79). London: Whurr Publishers Ltd.

Ehri, L. C. (1998). Grapheme-phoneme knowledge is essential for learning to read words in English. In Metsala, J. \& Ehri, L. (Eds.), Word recognition in beginning reading (pp. 3-40). Hillsdale, NJ: Erlbaum. 
Ehri, L. C., Wilce, L. S. (1979). The mnemonic value of orthography among beginning readers. Journal of Educational Psychology, 71, 26-40.

Ellis, A. W. (1994). Developmental disorders of reading writing. In Ellis, A. W. (Eds.). Reading, Writing and Dyslexia (pp. 93-111). Hove (UK): Lawrence Erlbaum Associates, Publishers.

Ellis, A. W., Flude, B. M., \& Young, A. W. (1987). "Neglect dyslexia" and the early visual processing of letters in words and nonwords. Cognitive Neuropsychology, 4, 439-464.

Ellis, N. (1991). Spelling and sound in learning to read. In Snowling, M. \& Thomson, M. ( $2^{\text {nd }}$ Eds.), Dyslexia Integrating Theory and Practice (pp. 80-94). London: Whurr Publishers Ltd.

Eviatar, A., Menn, L., \& Zaidel, E. (1990). Concreteness: Nouns, verbs and hemispheres. Cortex, 26, 611-624.

Eviatar, A. \& Zaidel, E. (1991). The effects of word length and emotionality on hemispheric contribution to lexical decision. Neuropsychologia, 29, 415-428.

Fabbro, F., Pesenti, S., Facoetti, A., Bonanomi, M., Libera, L., \& Lorusso, M. L. (2001). Cortex, 37, 65-73.

Fabri, M., Polonara, G., Quattrini, A., Salvolini, U., Pesce, M. D., \& Manzoni, T. (1999). Role of the corpus callosum in the somatosensory activation of the ipsilateral cerebral cortex: a fMRI study of callosotomized patients. European Journal of Neuroscience, 11, 3983-3994.

Fawcett, A. J., \& Nicolson, R. I. (1994). Naming speed in children with dyslexia. Journal of Learning Disabilities, 27, 641-646.

Felton, R. H. \& Wood, F. B. (1992). A reading level match study of nonword reading skills in poor readers with varying IQ. Journal of Learning Disabilities, 25, 318 326.

Fiez, J. A. \& Petersen, S. E. (1993). PET as part of an interdisciplinary approach to understanding processes involved in reading. Psychological Science, 4 (5), September. 
Fletcher, J. M., \& Satz, P. (1980). Developmental changes in the neuropsychological correlates of reading achievement: a six-year longitudinal follow-up. Journal of Clinical Neuropsychology, 2, 23-37.

Fletcher, J. M., Shaywitz, S. E., Shankweiler, D. P., Katz, L., Liberman, I. Y., Stuebing, K. K., Francis, D. J., Fowler, A. E., \& Shaywitz, B. A. (1994). Cognitive profiles of reading disability: Comparisons of discrepancy and low achievement definitions. Journal of Educational Psychology, 86(1), 6-23.

Fletcher, J. M., Taylor, H. G., Morris, R., \& Satz, P. (1982). Finger recognition skills and reading achievement: a developmental neuropsychological analysis. Developmental Psychology, 18, 124-132.

Flowers, D. L., Wood, F. B., Shankweiler, D. P, \& (1991). Regional cerebral blood flow correlates of language processes in reading disability. Archives Neurology, 48, 637-643.

Flynn, J. M., \& Boder, E. (1991). Clinical and electrophysiological correlates of dysphonetic and dyseidetic dyslexia. In Stein, J. F. (Eds.), Vision and visual dyslexia (pp. 121-131). Boca Raton, FL: CRC Press.

Flynn, J. M., \& Deering W. M. (1989). Subtypes of dyslexia: Investigation of Boder's system using quantitative neurophysiology. Developmental Medicine and Child Neurology, 31, 215-223.

Foorman, B. R. \& Liberman, D. (1989). Visual and phonological processing of words: A comparison of good and poor readers. Journal of Learning Disabilities, 22(6), 349-355.

Francis, W. N., \& Kućera, H. (1982). Frequency Analysis of English Usage, Lexicon and Grammar. Boston: Houghton Mifflin Company

Friedman, R. Ween, J. E., \& Albert, M. L. (1993). Alexia. In Heilman, K. M. \& Valestein, E. (Eds.), Clinical Neuropsychology ( $3^{\text {rd }}$ ed.) (pp.37-62), New York: Oxford University Press.

Frith, U. (1981). Experimental approaches to developmental dyslexia: An introduction. Psychological Research, 43, 97-109. 
Frith, U. (1985). Beneath the surface of developmental dyslexia. In Patterson, K. E., Marshall, J. C. \& Coltheart, M. (Eds.), Surface Dyslexia (pp. 301-330). London: Routledge \& Kegan Paul.

Frith, U. \& Snowling, M. (1983). Reading for meaning and reading for sound in autistic and dyslexic children. British Journal of Developmental Psychology, 1, 329-342.

Fuentes, L. J. \& Tudela, P. (1992). Semantic processing of foveally and parafoveally presented words in a lexical decision task. The Quarterly Journal of Experimental Psychology, $45 A$ (2), 299-322.

Funnell, E. (1983). Phonological processes in reading; new evidence from acquired dyslexia. British Journal of Psychology, 74, 159-180.

Funnell, M. G., Corballis, P. M., \& Gazzaniga, M. S. (2000). Insights into the functional specificity of the human corpus callosum. Brain, 123, 920-926.

Galaburda, A. M. (1993). Developmental dyslexia (editorial). Review of Neurology, 149, 1-3.

Galaburda, A. M., \& Kemper, T. L. (1979). Cytoarchitectonic abnormalities in developmental dyslexia: A case study. Annal of Neurology, 6, 94-100.

Galaburda, A. M., Corsiglia, J., Rosen, G. D., \& Sherman, G. F. (1987). Planum temporale asymmetry reappraisal since Geschwind and Levitsky. Neuropsychologia, 25, 853-868.

Galaburda, A. M., LeMay, M., Kemper, T., \& Geschwind, N. (1978). Right-left asymmetries in the brain: Structural differences between the hemispheres may underlie cerebral dominance, Science, 199, 852-856.

Galaburda, A. M., Sherman, G., Glenn, D. R., Aboitiz, F., \& Geschwind, N. (1985). Developmental dyslexia: Four consecutive patients with cortical anomalies. Annal Of Neurology, 18, August, 222-233.

Galin, D., Diamond, R, \& Herron, J. (1977). Development of crossed and uncrossed tactile localization on the fingers. Brain and Language, 4, 588-590. 
Galin, D., Johnstone, J., Nakel, L., \& Herron, J. (1979). Developmental of the capacity for tactile information transfer between hemispheres in normal children, Science, $204,1330-1332$.

Gathercole, S. E. \& Baddeley, A. D. (1989). The role of phonological memory in normal and disordered language development. In Lundberg, C. von Euler, I. \& Lennerstrand, G. (Eds.), Brain and Reading (pp. 245- 255). Yew York: Stockton Press.

Gathercole, S. E. \& Baddeley, A. D. (1990). Phonological memory deficits in language disordered children: Is there a causal connection? Journal of Memory and Language, 29, 336-360.

Gazzaniga, M. S., Le Doux, J. E., \& Wilson, D. H. (1977). Language, praxis, and the right hemisphere: Clues to some mechanisms of consciousness. Neurology, 27 (12), 1144-1147.

Geffen, G., Nilsson, J. Simpson, D. A., \& Jeeves, M. A. (1994). The development of interhemispheric transfer of tactile information in cases of callosal agenesis. In Lassonde, M. \& Jeeves, M. A. (Eds.), Callosal Agenesis, A Natural Split Brain (pp.185-198). New York: Plenum Press.

Geffen, G., Nilsson, J., Quinn, K., \& Teng., E. L. (1985). The effect of lesions of the corpus callosum on finger localization. Neuropsychologia, 23, 497-514.

Geoffroy, G. (1994). Other syndromes frequently associated with callosal agenesis (pp.55-62). In Lassonde, M. \& Jeeves, M. A. (Eds.), Callosal Agenesis: A Natural Split Brain? NY, London: Plenum Press.

Geschwind, N., \& Galaburda, A. M. (1985a). Cerebral lateralization: Biological mechanisms, associations, and pathology: I. A hypothesis and a program for research. Archives of Neurology, 42, 429-459.

Geschwind, N., \& Galaburda, A. M. (1985b). Cerebral hypothesis and a program for research Archives of Neurology, 42, 521-552. 
Geschwind, N., \& Galaburda, A. M. (1985c). Cerebral lateralization: Biological mechanisms, associations, and pathology: III. A hypothesis and a program for research. Archives of Neurology, 42, 634-657.

Geschwind, N., \& Levitsky, W. (1968). Human brain: Left-right asymmetries in temporal speech region. Science, 161, 186-187.

Geschwind, N.,\& Behan, P. (1982). Left-handedness: Association with immune disease, migraine, and developmental learning disorder. Processing of National_Academy of Science in U.S.A, 79, 5097-5100.

Giedd, J. N., Blumenthal, J., Jeffries, N. O., Rajapakse, J. C., Vaituzis, C., Liu, H., Berry, Y. C., Tobin, M., Nelson, J., Castellanos, Z. (1999). Development of the human corpus callosum during childhood and adolescence: A Longitudinal MRI study. Progressive Neuro-Psychopharmacology \& Biological Psychiatry, 23, 571588.

Giedd, J. N., Rumsey, J. M., Castellanos, F. X., Rajapakse, J. C., Kaysen, D., Vaituzis, A. C., Vauss, Y. C., Hamburger, S. D., \& Rapoport, J. L. (1996). A quantitative MRI study of the corpus callosum in children and adolescents. Developmental Brain Research 91, 274-280.

Gladstone, M, \& Best, C. T. (1985). Developmental dyslexia: The potential role of interhemispheric collaboration in reading acquisition. In Best, C.T., Hemispheric Function and Collaboration in the Child (pp. 87-118). Toronto: Academic Press.

Gladstone, M., Best, C. T., \& Davidson, R. J. (1989). Anomalous bimanual coordination among dyslexic boys. Developmental Psychology, 25, 236-246.

Gloning, I., Cloning, K., Haub, G, \& Quatember, R. (1969). Comparison of verbal behavior in right-handed and non-right-handed patients with anatomically verified lesions of one hemisphere. Cortex, 5, 43-52.

Goodale, M. A. \& Graves, J. A. (1982). Retinal locus as a factor in interocular transfer in the pigeon (pp. 211-240). In Ingle, D. J., Goodale, M. A., R., \& Mansfield, J. W. (Eds.), Analysis of Visual Behavior. Cambridge, MA: The MIT Press. 
Gordon, H. W. (1974). Auditory specialization of the right and left hemispheres. In Kinsbourne, M. \& Smith, W. L. (Eds.), Hemispheric Disconnection and Cerebral Function (pp. 126-136). Springfield, IL: Thomas.

Gordon, H. W. \& Sperry, R. W. (1969). Lateralization of olfactory perception in the surgically separated hemispheres of man. Neuropsychologia, 7, 111-120.

Goswami, U. \& Bryant, P. (1990). Phonological skills and learning to read. U.K.: Lawrence Erlbaum Associates Ltd.

Gough, P. B. \& Turner, W. E. (1986). Decoding, reading, and disability. RASE: Remedial and Special Education, 7, 6-10.

Gough, P. B. (1996). How children learn to read and why they fail. Annals of Dyslexia, $46,3-20$.

Greitman, L. R. \& Rozin, P. (1977). The structure and acquisition of reading, I: Relations between orthographies and the structure of language. In Reber, A. S. \& Scarborough, D. L. (Eds.), Towards a Psychology of Reading. Hillsdale, NJ: Lawrence Erlbaum Associates Inc.

Grigorenko, E. L., Wood, F. B., Meyer, M. S., \& Pauls, D. L. (2000). Chromosome 6p influences on different dyslexia-related cognitive processes: Further confirmation. American Journal of Humane Genetics, 66, 715-723.

Gross, K., Rothenberg, S., Schottenfeld, S., \& Drake, C. (1978). Duration thresholds for letter identification in left and right visual fields for normal and reading-disabled children. Neuropsychologia, 16, 709-715.

Gross-Glenn, K. Duara, R. Barker W.W., et al., (1991). Positron emission tomographic studies during serial word-reading by normal and dyslexic adults. Journal of Clinical Experimental Neuropsychology, 13, 531-544.

Gross-Glenn, K., \& Rothberg, S. (1984). Interhemispheric tactile transfer in dyslexics. International Journal of Neuroscience, 24, 23-35.

Gross-Glenn, K., Skottun, B. C., Glenn, W., Kushch, A., Lingua, R., Dunbar, M., Jallad, B., Lubs, H. A., Levin, B., Rabin, M., Parke, L. A., \& Duara, R. (1995). Contrast sensitivity in dyslexia. Visual Neuroscience, 12, 153-163. 
Grossi, D., Trojano, L., Chiacchi, L., Soricelli, A., Mansi, L., Postglione, A., \& Salvatore, M. (1991). Mixed transcortical aphasia: Clinical features and neuroanatomical correlates. A possible role of the right hemisphere. European Neurology, 31, 204-211.

Habib, M., Gayraud, D., Oliva, A., Regis, J., Salmon, G., \& Khalil, R. (1991). Effects of handedness and sex on the morphology of the corpus callosum: A study with brain magnetic resonance imaging. Brain and Cognition, 16, 41-61.

Hammill, D. D., Pearson, N. A., \& Wiederholt, J. L. (1996). Comprehensive Test of Nonverbal Intelligence. Austin, TX: PRO-ED.

Han, J. S., Benson, J. E., Kaufman, B, et al. (1985). MR imaging of pediatric cerebral abnormalities, Journal of Computer Assisting Tomography 9, 103-114.

Hécaen, H. \& Albert, M. L. (1978). Human Neuropsychology (pp.123-156). New York: Academic Press.

Hellige, J. B. (1983). The study of cerebral hemisphere differences: Introduction and overview. In Hellige, J. B. (Eds.) Cerebral Hemisphere Asymmetry: Method, Theory, and Application (pp. 1-17). New York: Praeger Publishers.

Hellige, J. B. (1993). Unity of thought and action: Varieties of interaction between the left and right cerebral hemispheres. Current Directions In Psychological Science, 21-25.

Hellige, J. B., Taylor, A. K., \& Eng, T. L. (1989). Interhemispheric interaction when both hemispheres have access to the same stimulus information. Journal of Experimental Psychology: Human Perception and Performance, 15(4), 711-722.

Hendriks, A. W. \& Kolk, H. J. J. (1997). Strategic control in developmental dyslexia. Cognitive Neuropsychology, 14(3), 321-366.

Hernanz-Schuman, M., Dohan, F. C. Jr. Cayea, P., Wallman, J., \& Teele, R. L. (1985). Sonographic appearance of callosal agenesis: correlation with radiographic and pathological findings. American Journal of NeuroRadiology, 6, 361-368. 
Hier, D. B. \& Mohr, J. P. (1977). Incongruous oral and written naming: evidence for a subdivision of the syndromes of Wernicke's aphasia. Brain and Language, 4, 115126.

Hier, D. B., LeMay, M., Rosenberger, P. B., \& Perlo, V. P. (1978). Developmental dyslexia. Archived Neurology, 35, 90-92.

Hilpert, P. L. \& Kurtz, A. B. (1990). Prenatal diagnosis of agenesis of the corpus callosum using endovaginal ultrasound. Journal of Ultrasound Medicine, 29, 474479.

Hinshelwood, J. (1907). Four cases of congenital word-blindness occurring in the same family. British Medical Journal, 2, 1229-32.

Hinshelwood, J. (1917). Congenital word-blindness. London: H. K. Lewis.

Holligan, C. \& Johnston, R. S. (1988). The use of phonological information by good and poor readers in memory and reading tasks. Memory and Cognition, 16, 522532.

Holloway, R. L., \& DE Lacoste, M. C. (1986). Sexual dimorphism in the human corpus callosum: an extension and replication study. Human Neurobiology, 5, 87-91.

Horwitz, B., Rumsey, J. M., \& Donohue, B. C. (1998). Functional connectivity of the angular gyrus in normal reading and dyslexia. Proceeding National Academy of Science U.S.A., 95, 8939-8944.

Howes, N. L., Bigler, E. D., Lawson, J. S. \& Burlingame, G. M. (1999). Reading disability subtypes and the test of memory and learning. Archives of Clinical Neuropsychology, 14 (3), 317-339.

Howell, D. (1987). Statistical methods for psychology ( $2^{\text {nd }}$ ed.). Boston: PWS-KENT Publishing company.

Hubel, D. H. (1988). Eye, brain, and vision. New York: Scientific American Library.

Hulme, C. \& Snowling, M. (1992). Deficits in output phonology: An explanation of reading failure? Cognitive Neuropsychology, 9 (1), 47-72. 
Hulme, C., Muter, V., \& Snowling, M. (1998). Segmentation does predict early progress in learning to read better than rhyme: A reply to Bryant. Journal of Experimental Child Psychology, 71, 39-44.

Humphreys, P., Kaufmann, W. E., \& Galaburda, A. M. (1990). Developmental dyslexia in women: Neuropathological findings in three cases. Annals of Neurology, 28, 727-738.

Hynd, G. W. (1992). Neurological aspects of dyslexia: Comment on the balance model. Journal of Learning Disability, 25, 110-112.

Hynd, G. W., Hall, J., Novey, E. S., Eliopulos, D., Black, K., Gonzalez, J. J., Edmonds, J. E., Riccio, C., \& Cohen, M. (1995). Dyslexia and corpus callosum morphology. Archive of Neurology, 52, 32-38.

Hynd, G. W., Semroud-Clikeman, M., Lorys, A. R., Novey, E. S., \& Eliopulos, D. (1990). Brain morphology in developmental dyslexia and attention deficit disorder/hyperactivity. Archives Neurology, 47, 919-926.

Innocenti, G. M. (1986). General organization of callosal connections in the cerebral cortex. In Jones, E. G. \& Peters, A. (Eds.), Cerebral Cortex, 5 (pp. 291-353). New York. Plenum Publishing Corporation.

Jackson, J. H. (1868). Selected Writings of John Hughlings Jackson. In Taylor, J. (Eds.) New York: Basic Books, 1958.

Jancke, L., Wunderlich, G., Schlaug, G., \& Steinmetz, H. (1997). A case of callosal agenesis with strong anatomical and functional asymmetries. Neuropsychologia, $35,1389-1394$.

Jeeves, M. A. (1969). A comparison of interhemispheric transmission times in acallosals and normals, Psychonomic Science, 16-245-246.

Jeeves, M. A., \& Silver, P. H. (1988). The formation of finger grip during prehension in an acallosal patient, Neuropsychologia, 26, 153-159.

Jeeves, M. A., \& Temple, C. M. (1987). A further study of language function in callosal agenesis. Brain and Language, 32, 325-335. 
Jeret, J. S., Serur, D., Wisniewski, K. W., \& Lubin, R. A. (1987). Clinicopathological findings associated with agenesis of the corpus callosum. Brain Development, 9 , 255-256.

Johannes, S., Kussmaul, C., \& Mangun, G. R. (1996). Developmental dyslexia: Passive visual stimulation provides no evidence for a magnocellular processing defect. Neuropsychologia, 34, 1123-1127.

Johnston, R., Anderson, M. \& Duncan, L. (1991). Phonological and visual segmentation problems in poor readers. In M. Snowling, \& M. Thomson (Eds.), Dyslexia Integrating Theory \& Practice (pp. 154-164). London: Whurr Publishers.

Jorm, A. (1983). Specific reading retardation and working memory: A review. British Journal of Psychology, 74, 311-342.

Kandel, E. R. (1991). Perception of motion, depth, and form. In Kandel, E. R., Schwartz, J. H., \& Jessell, T. M. (Eds.), Principles of Neural Science $\left(3^{\text {rd }}\right.$ ed.) (pp.441-466). Connecticut: Appleton \& Lange.

Katz, R. B, Shankweiler, D., \& Liberman, I. Y. (1981). Memory for item order and phonetic recoding in the beginning reader. Journal of Experimental Child Psychology, 32, 474-484.

Katz, R. B. (1986). Phonological deficiencies in children with reading disability: Evidence from an object-naming task. Cognition, 22, 225-257.

Keen, A. G., \& Lovegrove, W. J. (2000). Transient deficit hypothesis and dyslexia: examination of whole-parts relationship, retinal sensitivity, and spatial and temporal frequencies. Vision Research, 40, 705-715.

Kelly, J. P (1991). The neural basis of perception and movement. In Kandel, E. R. Schwartz, J. H., \& Jessell, T. M. (Eds.), Principles of Neural Science $\left(3^{\text {rd }} \mathrm{ed}.\right)$, (pp.283-295). Connecticut: Appleton \& Lange.

Kershner, J. R., Henninger, P., \& Cooke, W. (1984). Written recall induces a right hemisphere linguistic advantage for digits in dyslexic children. Brain and Language, 21, 105-122. 
Kertesz, A., Polk, M., Howell, J., \& Black, S. E. (1987). Cerebral dominance, sex, and callosal size in MRI. Neurology, 37, 1385-1388.

Kimura, D. (1961). Cerebral dominance and the perception of verbal stimuli. Canadian Journal of Psychology, 15, 166-171.

Kimura, D. (1967). Functional asymmetry of the brain in dichotic listening. Cortex, 3, 163-178.

Kinsbourne, M. (1975). The mechanisms of hemispheric control or the lateral gradient of attention. In Rabbitt, P. M. A. \& Dornic, S. (Eds.), Attention and performance. New York: Academic Press.

Kirtlery, C., Bryant, P., Maclean, M., \& Bradley, L. (1989). Rhyme, rime, and the onset of reading. Journal of Experimental Child Psychology, 48, 224-245.

Klicpera, C., Wolff, P. H. (1981). Bimanual coordination in adolescent boys with reading retardation. Developmental Medicine and Child Neurology, 23, 617-625.

Kochnower, J., Richardson, E., \& DiBenedetto, B. (1983). A comparison of phonic decoding ability of normal and learning disabled children. Journal of Learning Disabilities, 16, 348-351.

Koivisto, M \& Laine, M.(2000). Hemispheric asymmetries in activation and integration of categorical information. Laterality, 5(1), 1-21.

Kramer, J. H., Knee, K., \& Delis, D. C. (2000). Verbal memory impairments in dyslexia. Archives of Clinical Neuropsychology, 15 (1), 83-93.

Kubova, Z., Kuba, M., Peregrin, J., \& Novakova, V. (1995). Visual evoked potential evidence for magnocellular system deficit in dyslexia. Physiology Research, 44, 87-89.

Kussmaul, Z. (1877). Ziemsson's Cyeiloaia, 14.

Larsen, J. P., Hoien, T., \& Odegaard, H. (1992). Magnetic resonance imaging of the corpus callosum in developmental dyslexia. Cognitive Neuropsychology, 9(2), 123-134. 
Krutsch, A. J. \& McKeever, W. J. (1990). Cross-modal correlation of dichotic and tachistoscopic language laterality tasks: The importance of familial sinistrality. Brain and Language, 38, 384-397.

Larsen, J. P., Hoien, T., Lundberg, I., \& Odegaard, H. (1990). MRI evaluation of the size and symmetry of the Planum Temporale in adolescents with developmental dyslexia. Brain and Language, 39, 289-301.

Larsen, P. D. \& Osborn, A. G. (1982). Computerized tomographic evaluation of corpus callosum agenesis and associated malformations. Journal of Computer Tomography, 6, 225-230.

Lashley, K. (1950). In search of the engram. Symposia of the Society of Experimental Biology, 4, 454-484.

Lassonde, M. (1986). The facilitatory influence of the corpus callosum on intrahemispheric processing (pp. 385-401). In Lepore, F., Ptito, M., \& Jasper, H. H. (Eds.), Two Hemispheres-One Brain: Functions of the Corpus Callosum. New York: Alan R. Liss.

Lassonde, M., Sauerwein, H., McCabe, N., Laruencelle, L., \& Geoffroy, G. (1988). Extent and limits of cerebral adjustment to early section or congenital absence of the corpus callosum. Brain and Behaviour Research, 30, 165-181.

Lekowicz, N. D. (1980). Phonemic awareness training: What to teach and how to teach it. Journal of Educational Psychology, 72, 686-700.

Levine, S.C. \& Banich, M. T. (1982). Lateral asymmetries in the naming of words and corresponding line drawings. Brain and Language, 17, 34-45.

Levy, J. \& Trevarthen, C. (1976). Metacontrol of hemispheric function in human splitbrain patients. Journal of Experimental Psychology: Human Perception and Performance, 2 (3), 299-312.

Levy, J., Trevarthen, C., \& Sperry, R. (1972). Perception of bilateral chimeric figures following hemispheric disconnection. Brain, 95, 61-78. 
Levy-Agresti, J. \& Sperry, R. W. (1968). Differential perceptual capacities in major and minor hemispheres. Proceedings of the U.S. National Academy of Sciences, 61, 1151.

Liberman, I. Y., Shankweiler, D. Fischer, F. W., \& Carter, B. (1974). Explicit syllable and phoneme segmentation in the young child. Journal of Experimental Child Psychology, 18, 201-212.

Liberman, I. Y., Shankweiler, D., Liberman, A. M., Fowler, C., \& Fischer, F. W. (1977). Phonetic segmentation and recoding in the beginning reader. In A. S. Reber \& D. L. Scarborough (Eds.), Toward a Psychology of Reading (The Proceedings of the CUNY Conference. Hillsdale, NJ: Erlbaum.

Lindgren, S. D. (1978). Finger localization and the prediction of reading disability. Cortex, 14, 87-101.

Livingstone, M. \& Hubel, D. (1988). Segregation of form, color, movement, and depth: Anatomy, physiology, and perception. Science, 240, 740-749.

Livingstone, M., Rosen, G. D., Drislane, F. W., \& Galaburda, A. M. (1991). Physiological and anatomical evidence for a magnocellular defect in developmental dyslexia. Proceeding, National Academy of Science, USA, 88, 7943-7947.

Loring, D. W., Meador, K. J., \& Lee, G. P. (1989). Differential-handed response to verbal and visual spatial stimuli: evidence of specialized hemispheric processing following callosotomy. Neuropsychologia, 27, 811-827.

Lovegrove, W. J., Garzia, R. P, \& Nicholson, S. B. (1990). Experimental evidence for a transient system deficit in specific reading disability. American Optometric Association Journal, 61, 137-146.

Lovegrove, W. R., Martin, F., \& Slaghuis, W. (1986). A theoretical and experimental case for a visual deficit in specific reading disability. Cognitive Neuropsychology, $3,225-267$. 
Lundberg, I. (1989). Lack of phonological awareness: A critical factor in dyslexia. In Lundberg, C. von Euler, I. \& Lennerstran, G. (Eds.), Brain and Reading (pp. 221231). New York: Stockton Press.

Lundberg, I., Olofesson, A., \& Wall, S. (1980). Reading and spelling skills in the first school years predicted from phonemic awareness skills in kindergarten.

Scandinavian Journal of Psychology, 21, 159-173.

Macaruso, P., Locke, J. L., Smith, S. T., \& Powers, S. (1995). Short-term memory and phonological coding in developmental dyslexia. Journal of Neurolinguistics, 9(2), 135-146.

Mann, V. A. \& Liberman, I. Y. (1984). Phonological awareness and verbal short-term memory. Journal of Learning Disabilities, 17(10), 592-598.

Mann, V. A. \& Liberman, I. Y., \& Shankweiler, D. (1980). Children's memory for sentences and word strings in relation to reading ability. Memory and Cognition, 8, 329-335.

Mann, V. A. (1986). Phonological awareness: The role of reading experience. Cognition, 24, 65-92.

Martin, F., \& Lovegrove, W. J. (1987). Flicker contrast sensitivity in normal and specifically disabled readers. Perception, 16, 215-221.

Martin, J. H., Brust, J. C. M., \& Hilal, S. (1991). Imaging the living brain. In Kandel, E. R., Schwartz, J. H., \& Jessell, T. M. (Eds.), Principles of Neural Science $\left(^{\text {rd }}\right.$ ed.) (pp.309-324). Norwalk, Connecticut: Appleton \& Lange.

Mason, C. \& Kandel, E. R. (1991). Central Visual Pathways. In Kandel, E. R., Schwartz, J. H., \& Jessell, T. M. (Eds.), Principles of Neural Science ( $3^{\text {rd }}$ ed.) (pp.420-439). Connecticut: Appleton \& Lange.

Mattingly, I. G. (1972). Reading, the linguistic process and linguistic awareness. In Kavanagh, J. \& Mattingly, I. (Eds.), Language by ear and by eye (pp.133-147). Cambridge, MA: MIT Press.

McBride-Chang, C. \& Manis, F. R. (1996). Structural invariance in the association of naming speed, phonological awareness, and verbal reasoning in good and poor 
readers: A test of the double deficit hypothesis. Reading and Writing: An Interdisciplinary Journal, 8, 323-339.

McClearly, R. A. (1960). Type of response as a factor in interocular transfer in the fish. Journal of Comparative Physiology and Psychology, 53, 311-321.

McCusker, L. X., Hillinger, M. L., \& Bias, R. G. (1981). Phonological recoding and reading. Psychological Bulletin, 89, 217-245.

Mesulam, M. M. (1990). Large scale neurocognitive networks and distributed processing for attention, language and memory. Annal Neurology, 28, 587-613/

Miller, G. A. (1991). The science of words. New York: Scientific American Library.

Milne, R. D., Syngeniotis, A., Jackson, G., \& Corballis M.C. (2002). Mixed lateralization of phonological assembly in developmental dyslexia. Neurocase, 8 , 205-209.

Milner, A. D. (1994). Visual integration in callosal agenesis. In Lassonde, M. \& Jeeves, M. A. (Eds.), Callosal Agenesis A Natural Split Brain? (pp.171-183). New York: Plenum Press.

Milner, A. D., Jeeves, M. A., Silver, P. H., Lines, C. R., \& Wilson, J. (1985). Reaction times of lateralized visual stimuli in callosal agenesis: Stimulus and response factors. Neuropsychologia, 23, 323-331.

Milner, A. D., Lines, C. R. (1982). Interhemispheric pathways in simple reaction time to lateralized light flash. Neuropsychologia, 20, 171-179.

Milner, B., \& Kolb, B. (1985). Performance of complex arm movements and facialmovement sequences after cerebral commissurotomy. Neuropsychology, 23, 791799.

Moffat, S. D., Hampson, E., \& Lee, D. H. (1998). Morphology of the planum temporale and corpus callosum in left handers with evidence of left and right hemisphere speech representation. Brain, 121, 2369-2379.

Moore, L. H., Brown, W. S., Markee, T. E., Theberge, D. C., \& Zvi, J. C. (1996). Cortex, 32, 311-322. Fletcher, J. M., Taylor, H. G., Morris, R., \& Satz, P. (1982). 
Finger recognition skills and reading achievement: a developmental neuropsychological analysis. Developmental Psychology, 18, 124-132.

Morgan, P. (1896). A case of congenital word-blindness. British Medical Journal, 2, 1378.

Morris, D. W., Robinson, L., Turoc, D., Duke, M., Webb, V., Milham, C., Hopkin, E., Fernando, S., Easton, M., Hamshere, M., Williams, N., McGuffin, P., Stevenson, J., Krawczak, M., Owen, M. J., O’Donovan, C., \& Williams, J. (2000). Familybased association mapping provides evidence for a gene for reading disability on chromosome 15q. Human Molecular Genetics, 9 (5), 843-848.

Muter, V., Hulme, C., Snowling, M., \& Taylor, S. (1998). Segmentation, not rhyming, predicts early progress in learning to read. Journal of Experimental Child Psychology, 71, 3-27.

Myers, R. E., \& Sperry, R. W. (1958). Interhemispheric communication through the corpus callosum. Mnemonic carry-over between the hemispheres. Archives of Neurology and Psychiatry, 80, 298-303.

Neely, J. H. (1977). Semantic priming and retrieval from lexical memory: Roles of inhibitionless spreading activation and limited-capacity attention. Journal of Experimental Psychology: General, 106, 226-254.

Neely, J. H. (1991). Semantic priming effects in visual word recognition: A selective review of current findings and theories. In D. Besner \& G. W. Humphreys (eds.), Basic processes in reading, visual word recognition, pp. 264-336. Hillsdale, NJ: Lawrence Erlbaum.

Nobel, J. (1968). Paradoxical interocular transfer of mirror-image discrimination in the optic chiasm sectioned monkey. Brain Research, 10, 127-151.

O’Kusky, J., Strauss, E., Kosaka, B., Wada, J., Li, D., Druhan, M., \& Petrie, J. (1988). The corpus callosum is larger with right-hemisphere cerebral speech dominance. Annal of Neurology, 24, 379-383.

Oakhill, J. \& Kyle, F. (2000). The relation between phonological awareness and working memory. Journal of Experimental Child Psychology, 75, 152-164. 
Oldfield, R. C. (1971). The assessment and analysis of handedness: The Edinburgh Inventory. Neuropsychologia, 9, 97-113.

Olivares, R. O., Michalland, S., \& Aboitiz, F. (2000). Cross-species and intraspecies morphometric analysis of the corpus callosum. Brain Behavior and Evolution, 55, 37-43.

Olson, R. K., Kliegel, R., Davidson, B. J., \& Foltz, G. (1985). Individual and developmental differences in reading disability. In MacKinnon, G. E. \& Waller, T. G. (Eds.), Reading Research: Advances in Theory and Practice, vol. 4, (pp. 164). New York: Academic Press.

Oppenheim, J. S., Skerry, J. E., Tramo, M. J., \& Gazzaniga, M. S. (1989). Magnetic resonance imaging morphology of the corpus callosum in monozygotic twins. Annal of Neurology, 26, 100-104.

Ortells, J. J., Tudela, O., Noguera, C., \& Abad, M. J. F. (1998). Attentional orienting within visual field in a lexical decision task. Journal of Experimental Psychology: Human Perception and Performance, 24 (6), 1675-1689.

Orton, S. T. (1937). Reading, Writing and Speech Problems in Children. New York: W.W. Norton \& Company, Inc.

Ottawa-Carleton District School Board (2004). Retrieved February 14, 2004, from http://www.ocdsb.edu.on.ca/General Info/General.html.

Ottawa-Carleton Catholic School Board (2004). Retrieved February 14, 2004, from http://www.ocedsb.edu.on.ca/welcom.html.

Palmer, S. (2000). Phonological recoding deficit in working memory of dyslexic teenagers. Journal of Research in Reading, 23(1), 28-40.

Pandya, D. N., \& Seltzer, B. (1986). The topography of commissar fibers. In Lepore, F. Ptito, M. \& Jasper, H. (Eds.), Two Hemispheres-One Brain: Functions of the Corpus Callosum (pp. 47-73). New York. Alan R. Liss, Inc.

Patterson, K. E. \& Kay, J. (1982). Letter by letter reading; psychological descriptions of a neurological syndrome. Quarterly Journal of Experimental Psychology, 34A, 411-441. 
Patterson, K. E., Marshall, J. C., \& Coltheart, M. (1985). Surface dyslexia. Hillsdale, NJ: Erlbaum.

Paulesu, E., Frith, U. Snowling, M., Gallagher, A., Morton, J., Frackowiak, R. S. J., Frith, C. D. (1996). Is developmental dyslexia a disconnection syndrome? Evidence from PET scanning. Brain, 119, 143-157.

Pennington, B. F., Filipek, P. A., Lefly, D., Churchwell, J., Kennedy, D. N., Simon, J. H., Filley, c. M., Galaburda, A., Alarcon, M., \& DeFries, J. C. (1999). Brain morphometry in reading-disabled twins. Neurology, 53, 723-729.

Perfetti, C. A. (1992). The representation problem in reading acquisition. In Cough, P.B. \& Ehri, L.C. (Eds.), Reading Acquisition (pp. 145-174). Hillsdale, NJ, England: Lawrence Erlbaum Associates Inc.

Petersen, S. E., Fox, P. T., Posner, M. I., Mintun, M., \& Raichle, M. E. (1988). Positron emission tomographic studies of the cortical anatomy of single-word processing. Nature, 331, 585-589.

Poldrack, R. A., Temple, E., Protopapas, A., Nagarajan, S., Tallal, P., Merzenich, M. \& Gabrieli, J. D. E. (2001). Relations between the neural bases of dynamic auditory processing and phonological processing evidence from fMRI. Journal of Cognitive Neuroscience, 13 (5), 687-697.

Postman, L. J. \& Keppel, G. (1970). Norms of word association. NY: Academic Press. Pozzili, C., Bastianello, S., Bozzao, Pierallini, A., Glubilei, F., Argentino, C., \& Bozzao, L. (1994). No differences in corpus callosum size by sex and aging. Journal of Neuroimaging, 4 (4), 218-221.

Preilowski, B. (1972). Possible contributions of the anterior forebrain commissures to bilateral motor coordination. Neuropsychologia, 10, 267-277.

Preilowski, B. (1975). Bilateral motor interaction: Perceptual motor performance of partial and complete "split brain" patients. In Zulch, K. J., Creutzfeldt, O., \& Galabraith, C. (Eds.), Cerebral localization (pp. 115-132.) Berlin: Springer.

Pugh, K. R., Mencl, W. E., Jenner, A. R., Katz, L., Frost, S. J., Lee, J. R., Shaywitz, S. E., \& Shaywitz, B. A. (2000). Functional neuroimaging studies of reading and 
reading disability (developmental dyslexia). Mental Retardation and Developmental Disabilities Research Review, 6, 207-213.

Pugh, K. R., Mencl, W. E., Jenner, A. R., Katz, L., Frost, S. J., Lee, J. R., Shaywitz, S. E., \& Shaywitz, B. A. (2000). Functional neuroimaging studies of reading and reading disability (developmental dyslexia). Mental Retardation and Developmental Disabilities Research Reviews, 6, 207-213.

Quinn, K \& Geffen, G. (1986). The development of tactile transfer of information. Neuropsychologia, 24, 793-804.

Rack, J. Snowling, M., \& Olson, R. (1992). The nonword reading deficit in developmental dyslexia: A review. Reading Research Quarterly, 27 (1), 29-53.

Rack, J., Hulme, C., Snowling, M., \& Wightman, J. (1994). The role of phonology in young children learning to read words: The direct-mapping hypothesis. Journal of Experimental Child Psychology, 57, 42-71.

Reber, A. S. (1995). The Penguin Dictionary of Psychology ( $2^{\text {nd }}$ Eds. $)(p p .264)$. London: Penguin Books.

Reynolds, D. McO \& Jeeves, M. A. (1974). Further studies of crossed and uncrossed pathway responding in callosal agenesis - reply to Kinsbourne and Fisher. Neuropsychologia, 12, 287-225.

Ridder III, W. H., Borsting, E. \& Banton, T. (2001). All developmental dyslexic subtypes display an elevated motion coherence threshold. Optometry and Vision Science, 78(7), 510-517.

Ridder III, W. H., Borsting, E., Cooper, M., McNeel, B., \& Hauing, E. (1997). Not all dyslexics are created equal. Optometry and Vision Science, 74, 99-104.

Robichon, F., \& Habib, M. (1998). Abnormal callosal morphology in male adult dyslexics: Relationships to handedness and phonological abilities. Brain and Language, 62, 127-146.

Robichon, F., Bouchard, P., Demonet, J., \& Habib, M. (2000). Developmental dyslexia: Re-Evaluation of the corpus callosum in male adults. European Neurology, 43, 233-237. 
Robichon, F., Levrier, O., Farnarier, P., \& Habib, M. (2000). Developmental dyslexia: atypical cortical asymmetries and functional significance. European Journal of Neurology, 7, 35-46.

Rosen, G. D., Sherman, G. F., \& Galaburda, A. M. (1993). Dyslexia and brain pathology: experimental animal models. In Galaburda, A. M. (Eds.), Dyslexia and Development, Neurobiological Aspects of Extra-Ordinary Brains (pp. 89-111). Massachusetts: Harvard University Press.

Rousselle, C., \& Wolff, P. H. (1991). The dynamics of bimanual coordination in developmental dyslexia. Neuropsychologia, 29, 907-924.

Rudel, R. G. (1985). Hemispheric asymmetry and learning disabilities: Left, right or in between? In Best, C. T. (Eds.), Hemispheric function and collaboration in the child (pp.275-308). San Francisco: Academic Press.

Rumsey, J M., Andreason, P, Zametkin, A. J., Aquino, T., King A. C., Hamburger S. D.(1992). Failure to activate the left temporo parietal cortex in dyslexia: An oxygen 15 positron emission tomographic study. Archive Neurology, 49, 527-534.

Rumsey, J. M., Casanova, M., Mannheim, G. B., Patronas, N., De Vaughn, N., Hamburger, S. D. , \& Aquino, T. (1996). Corpus callosum morphology, as measured with MRI, in dyslexic men. Biological Psychiatry, 39, 769-775.

Rumsey, J. M., Donohue, B. C., Brady, D. R., Nace, K., Giedd, J. N., \& Andreason, P (1997). A magnetic resonance imaging study of planum temporale asymmetry in men with developmental dyslexia. Archives Neurology, 54, 1481-1489.

Rutter, M., \& Yule, W. (1975). The concept of specific reading retardation. Journal of Child Psychology and Psychiatry, 16, 181-197.

Saffran, E. M. (1985). Lexicalization and reading performance in surface dyslexia. In Surface Dyslexia, K. E. Patterson, J. C. Patterson, J. C. Marshall, \& M. Coltheart (eds.). London: Lawrence Erlbaum Associates.

Sandri, F., Pilu, G., Cerisoli, M., Bovicelli, L., Alvisi, C., \& Salvioli, G. P. (1988). Sonographic diagnosis of agenesis of the corpus callosum in the fetus and newborn infant, American Journal of Perinatology, 5, 226-231. 
Sasanuma, S. (1974). Kanji versus Kana processing in alexia with transient agraphia: a case report. Cortex, 10, 89-97.

Sasanuma, S. (1985). Surface dyslexia and dysgraphia: how are they manifested in Japanese? In Patterson, K. E., Marshall, J. C., \& Coltheart, M. (Eds.), Surface Dyslexia. London: Lawrence Erlbaum Associates.

Sauerwein, H. C., \& Lassonde, M. (1994). Cognitive and sensori-motor functioning in the absence of the corpus callosum: Neuropsychological studies in callosal agenesis and callosotomized patients. Behavioural Brain Research, 64, 229-240.

Schacter, S. C. (1993). Studies of handedness and anomalous dominance: Problems and progress. In Galaburda, A. M. (Eds.), Dyslexia and development neurobilogical aspects of extra-ordinary brains (pp.269-296). Massachusetts: Harvard University Press.

Schonell, F., \& Goodacre, E. (1971). The psychology and teaching of reading, $\left(5^{\text {th }} \mathrm{ed}.\right)$. London: Oliver \& Boyd.

Schultz, R. T., Cho, N. K., Staib, L. H., Kier, L. E., Fletcher, J. M., Shaywitz, S. E., Shankweiler, D. P., Katz, L., Gore, J. C., Duncan, J. S., Shaywitz, B. A. (1994). Brain morphology in normal and dyslexic children: The influence of sex and age. Annal of Neurology, 35, 732-742.

Sergent, J. (1990). Furtive incursions into bicameral minds. Brain, 113, 537-568.

Serur, D., Jeret, J. S., \& Wisniewski, K. (1988). Agenesis of the corpus callosum: Clinical, neuroradiological and cytogenetic studies. Neuropediatrics, 19, 87-91.

Seymour, P. H. K. (1986). Beginning reading without phonology, Cognitive Neuropsychology, 3, 1-36.

Shallice, T. \& Warrington, E. K. (1980). Single and multiple component central dyslexic syndromes. In Coltheart, M., Patterson, K. E. \& Marshall, J. C. (Eds.), Deep Dyslexia. London: Routledge and Kegal Paul.

Shankweiler, D., Liberman, I. Y., Mark, L., Fowler, C. A., \& Fischer, F. W. (1979). The speech code and learning to read. Journal of Experimental Psychology: Human Learning and Memory, 5, 531-545. 
Shapley, R., Kaplan, E., \& Soodak, R. (1981). Spatial summation and contrast sensitivity of $\mathrm{X}$ and $\mathrm{Y}$ cells in the lateral geniculate nucleus of the macaque. Nature, 292, 543-545.

Share, D. L. (1995). Phonological recoding and self-teaching. Cognition, 55(2), 151218.

Shaywitz, B. A., Shaywitz, S. E., Pugh, K. R., Constable, R. T., Skudlarskl, P., Fulbright, R. K., Bronen, R. A., Fletcher, J. M., Shankweller, D. P., Katz, L., \& Gore, J. C. (1995). Sex differences in the functional organization of the brain for language. Nature, 373, 607-609.

Shaywitz, B. A., Shaywitz, S. E., Pugh, W. E., Mencl, R. K., Fulbright, R. K., Skudlarski, P., Constable, R. T., Marchione, K. E., Fletcher, J. M., Lyon, G. R., \& Gore, J. C. (2002). Disruption of posterior brain systems for reading in children with developmental dyslexia. Society of Biological Psychiatry, 52, 101-110.

Sherman, G. F., Galaburda, A. M., \& Geschwind, N. (1983). Ecropic neurons in the brain of the autoimmune mouse: A neuropathological model of dyslexia? Society of Neuroscience Abstract, 9, 939.

Siegel, L. S. \& Ryan, E. B. (1988). Development of grammatical sensitivity, phonological, and short-term memory skills in normally achieving and learning disabled children. Developmental Psychology, 24, 28-37.

Sieroff, E. (1991). Perception of visual letter strings in a case of left neglect: Manipulation of the word form. Brain and Language, 41(4). 565-589.

Silver, P. H., \& Jeeves, M. A. (1994). Motor coordination in callosal agenesis. In Lassonde, M. \& Jeeves, M. A. (Eds.), Callosal Agenesis A Natural Split Brain? (pp.207-219). New York: Plenum Press.

Simos, P. G., Fletcher, J. M., Bergman, E., \& Panicolauou, A. C. (2000). Cerebral mechanisms involved in word reading in dyslexic children: A magnetic source imaging approach. Cerebral Cortex, 10(8), 809-816. 
Sklar, B., Hanley, J., \& Simmons, W. W. (1973). A computer analysis of EEG spectral signatures from normal and dyslexic children. Transactions on Biomedical Engineering, 20, 20-26.

Skottun, B. C. (1997). Letter to the Editor: Some remarks on the Magnocellular deficit theory of dyslexia. Vision Research, 37, 965-966.

Skottun, B. C. (2000). On the conflicting support for the magnocellular-deficit theory of dyslexia Response to Stein, Talcott and Walsh (2000). Trends in Cognitive Sciences, 211-212.

Slaghuis, W. L. \& Ryan, J. H. (1999). Spatio-temporal contrast sensitivity, coherent motion, and visible persistence in developmental dyslexia. Vision Research, 39, 651-668.

Smith, S. D. \& Goldgar, D. E. (1986). Single gene analyses and their application to learning disabilities. In Smith, S. D. (Eds.), Genetics and Learning Disabilities. San Diego: College-Hill Press.

Snowling, M. (1981). Phonemic deficits in developmental dyslexia. Psychological Research, 43, 219-234.

Snowling, M. J. (1980). The development of grapheme-phoneme correspondence in normal and dyslexic readers. Journal of Experimental Child Psychology, 29, 294305.

Snowling, M. (1987). Dyslexia A cognitive developmental perspective. Oxford: Basil Blackwells Inc.

Snowling, M. (1995). Phonological processing and developmental dyslexia. Journal of Research in Reading, 18, 132-138.

Snowling, M. (2000). Dyslexia. MA: Blackwell Publishers Ltd.

Snowling, M. \& Frith, U. (1981). The role of sound, shape and orthographic cues in early reading. British Journal of Psychology, 72, 83-87.

Snowling, M. \& Hulme, C. (1994). The development of phonological skills. Transaction of the Royal Society of Britain, 346, 21-28. 
Soderbergh, R. (1971). Reading in early childhood: A linguistic study of a preschool child's gradual acquisition of reading ability. Stockholm: Almqvist $\&$ Wilksell.

Sperry, R. W. (1961). Cerebral organization and behavior. Science, 133, 1747-1757.

Sperry, R. W. (1962). Some general aspects of interhemispheric integration. In Mountcastle, V. B. (Eds.), Interhemispheric Relatings and Cerebral Dominance, (p.43-49). Baltimore: Md. Johns Hopkins Press.

Sperry, R. W. (1968). Hemispheric disconnection and unity in conscious awareness. American Psychology, 23, 723-733.

Sperry, R. W. (1974). Lateral specialization in the surgically separated hemispheres. In Schmitt, F. O. \& Worden, F. G. (Eds.), The Neurosciences: Third Study Program. (pp. 5-19). Cambridge: MIT Press.

Sperry, R. W. (1975). In search of psyche. In Worden, F. G. , Swazey, J. P., \& Adelman, G. (Eds.), The Neurosciences: Paths of Discovery (pp. 425-434). Cambridge: MIT Press.

Sperry, R. W. (1982). Some effects of disconnecting the cerebral hemispheres. Science, 217, 97-136.

Sperry, R. W., Gazzaniga, M. S., \& Bogen, J. E. (1969). Interhemispheric relationships: the neocortical commissures; syndromes of hemispheric disconnection. In Vinken, P. J. \& Bruyn, G. W. (Eds.), Handbook of Clinical_Neurology, 4 (pp. 273-290). Amsterdam: North-Holland.

Sprenger-Charolles, L., Cole, P., Lacert, P., \& Serniclaes, W. (2000). One subtype of developmental dyslexia: Evidence from processing time and accuracy scores. Canadian Journal of Experimental Psychology, 54, 87-102.

Springer, P. S. \& Deutsch, G. (1989). A historical overview of clinical evidence for brain asymmetries. In their book, Left Brain, Right Brain ( $3^{\text {rd }}$ ed.) (pp.13-14).

Stančák, Jr., A., Lucking, C. H., \& Kristeve-Feige, R. (2000). Lateralization of movement-related potentials and the size of corpus callosum. Cognitive Neuroscience and Neuropsychology, 11(27), 329-332. 
Stanovich, K. E. (1982). Individual differences in the cognitive processes of reading: I. Word decoding. Journal of Learning Disabilities, 15, 485-493.

Stanovich, K. E. (1991). Discrepancy definitions of reading disability: Has intelligence led us astray? Reading Research Quarterly, 26, 1-29.

Stanovich, K. E., Cunnigham, A. E., \& Cramer, B. B. (1984). Assessing phonological awareness in kindergarten children: Issues of task comparability. Journal of Experimental Child Psychology, 38, 175-190.

Stanovich, K. E., Cunningham, A. E., \& Feeman, D. J. (1984). Intelligence, cognitive skills, and early reading progress. Reading Research Quarterly, 19. 120-139.

Stanovich, K. E. \& Siegel, L. S. (1994). The phenotypic performance profile of readingdisabled children: a regression-based test of the phonological core variable difference model. Journal of Educational Psychology, 86, 1-30.

Stein, J \& Walsh, V. (1997). To see but not to read; the magnocellular theory of dyslexia. Trend Neuroscience, 20, 147-152

Steinmetz, H., Jäncke, L., Kleinschmidt, A., Schlaug, G., Volkmann, J., \& Huang, Y. (1992). Sex but no hand difference in the isthmus of the corpus callosum. Neurology, 42, 749-752.

Steven, A. S. \& Bruce, A. M. (1994). Defining phonological awareness and its relationship to early reading. Journal of Educational Psychology, 86, 221-234.

Stevens, J. (1996). Applied multivariate statistics for the social sciences ( $3^{\text {rd }}$ ed.). Mahwah, NJ: Lawrence Erlbaum Associates.

Stickles, J.L., Schilmoeller, G. L., \& Schilmoeller, K. J. (2002). International Journal of Disability, Development and Education, 49(4), 367-383.

Sugishita, M, Iwata, M. Toyokura, Y., Yoshioka, M., \& Yamada R. (1978). Reading of ideograms and phonograms in Japanese patients after partial commissurotomy. Nerupsychologia, 16. 417-426.

Sugishita, M, Otomo, K., Kabe, S., \& Yunoki, K. (1992). A critical appraisal of neuropsychological correlates of Japanese ideogram (Kanji) and phonogram (Kana) reading. Brain, 115, 1563-1585. 
Sugishita, M. Yoshioka, M., \& Kawamura, M. (1986). Recovery from hemialexia. Brain and Language, 29, 106-118.

Sullivan, M. S., \& Hamilton, C. R. (1973). Interocular transfer of reversed and nonreversed discriminations via the intact commissure of monkeys. Physiology and Behavior, 10, 355-359.

Summerfield, B. C., \& Michie, P. T. (1993). Processing of tactile stimuli and implications for the reading disability. Neuropsychologia, 31, 965-976.

Swan, D. \& Goswami, U. (1997). Picture naming deficits in developmental dyslexia: The phonological representation hypothesis. Brain and Language, 56, 334-353.

Tabachnick, B. G. \& Fidell, L. S. (1996). Using multivariate statistics ( $3^{\text {rd }}$ ed.). HarperCollins College Publishers.

Talcott, J. B., Hansen, P. C., Willis-Owen, C., McKinnell, I. W., Richardson, A. J., \& Stein, J. F. (1998). Visual magnocellular impairment in adult developmental dyslexics. Neuro-ophthalmology, 20, 187-201.

Tassinari, G., Aglioti, S., Pallini, R., Berlucchi, G., \& Rossi, G. F. (1994). Interhemispheric integration of simple visuomotor responses in patients with partial callosal defects. Behavioural Brain Research, 64, 141-149.

Temple, C. M. \& Ilsley, J. (1994). Sounds and shapes: Language and spatial cognition in callosal agenesis. In Lassonde, M. \& Jeeves, M. A. (Eds.), Callosal Agenesis: A Natural Split Brain? (pp.261-273). New York: Plenum Press.

Temple, C. M. \& Marshall, J. C. (1983). A case study of developmental phonological dyslexia. British Journal of Psychology, 74, 517-533.

Temple, C. M. (1984). Surface dyslexia in a child with epilepsy. Neuropsychologia, 22, 569-576.

Temple, C. M., Jeeves, M. A., \& Vilarroya, O. (1989). Ten pen men: Rhyming skills in two children with callosal agenesis. Brain and Language, 37, 348-564.

Temple, C. M., Jeeves, M. A., \& Vilarroya, O. (1990). Reading in callosal agenesis. Brain and Language, 39, 235-253. 
Tomasch, J. (1954). Size, distribution and number of fibers in the human corpus callosum. Anatomical Record. 119, 119-135.

Torgesen, J. K. (1988). Studies of children with learning disabilities who perform poorly on memory span tasks. Journal of Learning Disabilities, 21(10), 605-612.

Torgesen, J. K., Wagner, R. K., \& Rashotte, C. A. (1999). Test of Word Reading Efficiency. Texas: Pro-Eds.

Toyama, K., Tokashiki, S. \& Matsumi, K. (1969). 'Synaptic action of commissural impulses upon association efferent cells in cat visual cortex', Brain Research, 14, 518-520.

Treiman, R. \& Hirsh-Pasek, K. (1985). Are there qualitative differences between dyslexic and normal readers? Memory and Cognition, 13, 357-364.

Treiman, R. (1985). Onsets and rimes as units of spoken syllables: Evidence from children. Journal of Experimental Child Psychology, 39, 161-181.

Van der Leij, A.. \& Van Daal, V. H. P. (1999). Automatization aspects of dyslexia: Speed limitations in word identification, sensitivity to increasing task demands, and orthographic compensation. Journal of learning disabilities, 32, 417-428.

Van Wagene, W. P. \& Herren, R. Y. (1940). Surgical division of commissar pathways in the corpus callosum: Relation to spread of an epileptic attack. Archives of Neurology and Psychiatry, 44, 740-759.

Vandervelden, M. C. \& Siegel, L. S. (1995). Phonological recoding and phoneme awareness in early literacy: A developmental approach. Reading Research Quarterly, 30 (4), 854-875.

Vellutino, E. R., \& Scanlon, D. M. (1987). Phonological coding, phonological awareness, and reading ability: Evidence from a longitudinal and experimental study. Merrill Palmer Quarterly, 33, 321-364.

Vellutino, F. R. (1979). Dyslexia: Theory and research. Cambridge, MA: MIT Press.

Victor, J. D., Conte, M. M., Burton, L., \& Nass, R. D. (1993). Visual evoked potentials in dyslexics and normals: Failure to find a difference in transient or steady-state responses. Visual Neuroscience, 10, 939-946. 
Vidyasagar, T. R. \& Pammer, K. (1999). Impaired visual search in dyslexia relates to the role of the magnocellular pathway in attention. NeuroReport, 10, 1283-1287.

Wada, J. A., Clarke, R., Hamm, A. (1975). Cerebral hemispheric asymmetry in humans. Archives of Neurology, 32, 239-246.

Wagner, R. K. \& Torgesen, J.K. (1987). The nature of phonological processing and its causal role in the acquisition of reading skills. Psychological Bulletin, 101 (2), 192-212.

Watson, R. T. \& Heilman, K. M. (1983). Callosal apraxia. Brain, 106, 391-403.

Wechsler, D. (1991). Wechsler Intelligence Scale for Children-Third Edition. San Antonio: Psychological Corp.

Wechsler, D. (1981). Wechsler Adult Intelligence Scale-Third Edition. New York: The Psychological Corp.

Weis, S, Weber, G., Wenger, E., \& Kimbacher, M. (1989). The controversy about a sexual dimorphism of the human corpus callosum. International Journal of Neuroscience, 47, 169-173.

Wennekes, M. J. \& Njiokoktjien, C. (1991). Developmental interhemispheric disconnection and dyslexia. In Ramaekers, G. \& Njiokiktjien, C. (Eds.), The Child's Corpus Callosum. Amsterdam: Suyi PUblications.

Wernicke, K. (1874). Der Aphasische Symptomencomplex. Eine Psychologiche Studie auf Anatomischer Basis (The aphasia symptom complex: A psychological study on an anatomical basis). Breslau: M. Cohn \& Weigart (Reprinted 1977 in Wernicke's On Aphasia: A course book and reviews (G. E. Eggert, Trans \& Commentator, 99, 91-144).

Wickens, D. D. (1972). Characteristics of word encoding. In Melton, A. \& Martin, E. (Eds.), Coding processes in human memory, (pp. 191-215). Washington, D. C.: Winston.

Wiederholt, J. L., \& Bryant, B. R. (1992). Gray Oral Reading Tests-Third Edition. Austin, TX: PRO-ED. 
Wilding, J. (1989). Developmental dyslexics do not fit in boxes: Evidence from the case studies. European Journal of Cognitive Psychology, 1 (2), 105-127.

Williams, M. J., Stuart, G. W., Castles, A., \& McAnally, K. I. (2003). Contrast sensitivity in subgroups of developmental dyslexia. Vision Research, 43, 467-477.

Wimmer, H., Mayringer, H., \& Landerl, K. (1998). Poor reading: A deficit in skillautomatization or a phonological deficit? Scientific Studies of Reading, 2 (4), 321-340.

Wisniewski, K. W., \& Jeret, J. S. (1994). Callosal agenesis: Review of clinical, pathological, and cytogenetic features. In Lassonde, M. \& Jeeves, M A (Eds.), Callosal Agenesis: A Natural Split Brain? (pp.1-6). New York: Plenum Press.

Witelson, S. F. (1985). The brain connection: The corpus callosum is larger in lefthanders. Science, 229, 665-668.

Witelson, S. F. (1989). Hand and sex differences in the isthmus and genu of the human corpus callosum: a postmortem morphological study. Brain, 112, 799-835.

Wolff, P. H., Cohen, C., \& Drake, C. (1984). Impaired motor timing control in specific reading retardation. Neuropsychologia, 22, 587-600.

Wolff, P. H., Michel, G. F., \& Drake, C. (1990). Rate and timing precision of motor coordination in developmental dyslexia. Developmental Psychology, 26, 349-359.

Woodcock, R. W. (1987). Woodcock Reading Mastery Tests. Circle Pines, Minn.: American Guidance Service, Inc.

Yap, R. L., \& Van der Leij, A. (1994). Testing the automatization deficit hypothesis of dyslexia via a dual-task paradigm. Journal of Learning Disabilities, 27, 660-665.

Yeni-Komshian, G. H., Isenberg, D. \& Colberg, H. (1975). Cerebral dominance and reading disability: left visual field deficit in poor readers. Neuropsychologia, 13, 83-94.

Yoshii, F., Barker, W., Apicella, A., Chang, J., Sheldon, J., \& Duara, R. (1986). Measurements of the corpus callosum (CC) on magnetic resonance (MR) scans: Effects of age, sex, handedness, and disease. Neurology, Cleveland, 36, Supplement, 1, 133. 
Yule, W., Rutter, M., Berger, M. \& Thompson, J. (1974). Over and under achievement in reading: Distribution in the general population. British Journal of Educational Psychology, 44, 1-12.

Zaidel, E. (1979). Performance on the ITPA following cerebral commissurotomy and hemispherectomy. Neuropsychologia, 17, 259-280.

Zaidel, E. (1983). Disconnection syndrome as a model for laterality effects in the normal brain. In J. B. Hellige (Eds.) Cerebral Hemisphere Asymmetry: Method, Theory, and Application (pp. 95-151). New York: Praeger Publishers.

Zaidel, E. (1995). Interhemispheric transfer in the split brain: long-term status following complete cerebral commissurotomy. In Davidson, R. J. \& Hugdahl, K. (Eds.) Brain Asymmetry (pp. 491-532). Cambridge, MA: the MIT Press.

Zaidel, E., \& Sperry, R. W. (1977). Some long-term motor effects of cerebral commissurotomy in man. Neuropsychologia, 15, 193-204.

Zaidel, E \& Peters, A. M. (1981). Phonological encoding and ideographic reading by the disconnected right hemisphere: Two case studies. Brain and Language, 14 (2), 205-234.

Zaidel, D., \& Sperry, R. W. (1974). Memory impairment after commissurotomy in man. Brain, 97, 263-272.

Zung, B. J. (1986). Cognitive-academic correlates of finger localization in right-handed kindergarten girls. Perceptual and Motor Skills, 62, 227-234. 
Appendices 


\section{List of Appendices}

Appendix A

Tachistoscopic Technique (Visual Half-Field Presentation)

Appendix B

6

Computerized Axial Tomography (CT)

Appendix C

8

Magnetic Resonance Imaging (MRI)

Appendix D

Positron Emission Tomography (PET)

Appendix E

Functional Magnetic Resonance Imaging (fMRI)

Appendix F

Test of Word Reading Efficiency (TOWRE)

Sight Word Efficiency (SWE)

Phonetic Decoding Efficiency (PDE)

Appendix G

Non-standardized Vocabulary List (Castles \& Coltheart, 1993)

Appendix H

Example of the Test of Non-Verbal Intelligence-3 (TONI-3)

Appendix I

Parent Questionnaire

Appendix J

Recruitment Announcement Published in a Monthly Bulletin of the Learning Disabilities Association-Ottawa

Appendix K

Student Questionnaire 
Edinburgh Handedness Inventory (Oldfield, 1971)

Questions

Appendix M

Finger Localization Protocol

Appendix N

Stimulus Lists for Experiment 2 (Rhyming Judgment Task)

Appendix O

A Pilot Study: Test-Retest Reliability

Appendix P

Stimulus Lists for Experiment 3 (Lexical Decision Task)

Appendix Q

A Pilot Study: Stimulus Lists Used in Experiment 3

Appendix R

List of Abbreviations 


\section{Appendix A}

\section{Tachistoscopic Technique (Visual Half-Field Presentation)}

This experimental technique allows us to study the functional differences between the two cerebral hemispheres and also is used to assess interhemispheric connectivity associated with particular verbal and nonverbal cognitive tasks, such as word recognition or spatial orientation tasks (Sergent, 1986, 1987). As shown in Figure 1, in the visual system, when looking ahead and fixating on one point, information to the right of the fixation, known as the right visual field (RVF), is projected in the left hemisphere through the optic chiasm, and information to the left of the fixation, known as the left visual field (LVF) is directed to the right hemisphere via the optic chiasm, and both pieces of information can communicate through the corpus callosum (Springer \& Deutsch, 1989). A critical fact is that information presented in each visual field (except for peripheral vision, which projects its image in the ipsilateral hemisphere) is confined to the contralateral hemisphere at the primary visual cortex. Thus, the visual half-field presentation technique is also referred to as the half visual presentation or divided visual field technique (Banich, 1997). It is also sometimes referred to as the tachistoscopic technique because a machine called a tachistoscope was used before the existence of personal computers (Levy, \& Trevarthen, 1976; Levy, Trevarthen, \& Sperry, 1972).

There are two conditions for the presentation: unilateral and bilateral. In the unilateral presentation, a test stimulus is presented within one VF. It is frequently used to study how well each hemisphere can respond to the stimulus independently. In the bilateral presentation, a stimulus (the same/different) is presented in the left and right VF 
simultaneously. This method allows us to investigate an interaction of two hemispheres, specifically the integration function of the corpus callosum.

Several conditions are essential for the procedure. First, a stimulus must be presented at less than $200 \mathrm{~ms}$ to avoid eye movements (Banich, 1997). If eye movements occur, a new fixation will provide different information to the hemisphere. Second, the position of the stimulus needs to be at least $1.5-2.5^{\circ}$ lateral from the fixation to ensure information is initially projected in the contralateral hemisphere, even though slight eye movements occur (Banich, 1997). Third, if the stimulus is a word, the length of the word needs to be controlled, because five-syllable words take more time to scan and are responded to less accurately than four-syllable words. This typically occurs with words presented on the left VF for two reasons: (1) because English words are scanned from left to right, and thus, the initial part of a word provides more information than the end of the word (Brysbaert \& D'Ydewalle, 1990), and (2) the longer a word is, the farther the initial part of the word is away from the fovea, where visual acuity is the best (Hubel, 1988). Fourth, the viewing distance should be constant (approximately $35 \mathrm{~cm}$ to $70 \mathrm{~cm}$ depending on the size of screen) (Eglin, \& Hunter, 1990). In addition to these considerations, the duration of fixation, the word frequency and visual complexity, and the brightness of the screen should be controlled. The proposed thesis project will use the visual half-field presentation. 


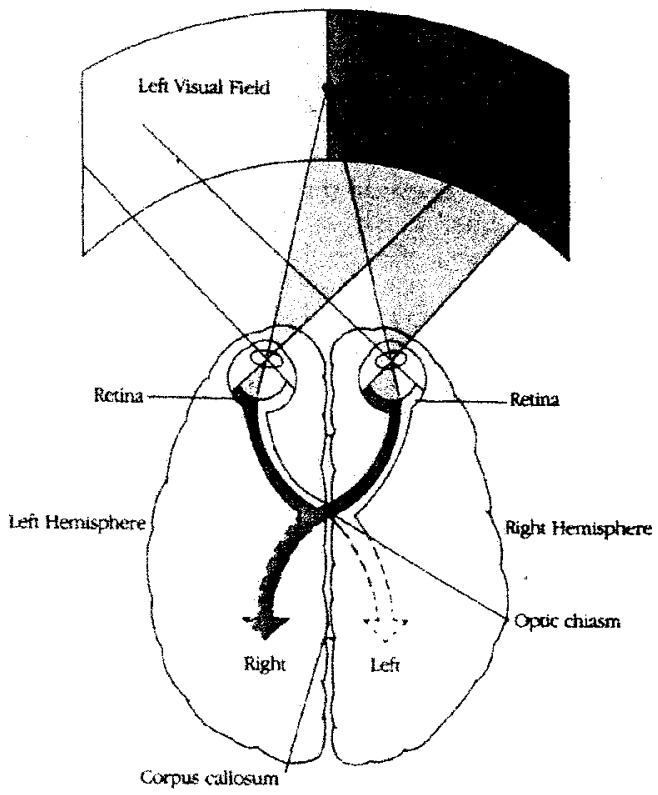

Figure 1. Visual pathways to the hemispheres. When fixating on a point, each eye sees both visual fields but sends information about the right visual field only to the left hemisphere. The visual areas of the left and right hemispheres normally communicate through the corpus callosum. If the callosum is cut and the eyes and head are kept from moving, each hemisphere can see only half of the visual world (Springer \& Deutsch, "Left Brain, Right Brain, $3^{\text {rd }}$ Ed., 1989). 


\section{Appendix B}

\section{Computerized Axial Tomography (CT)}

A CT scan projects brain structures using differences in brain density by x-ray. That is, bone, the densest part of brain structures, appears white, while cerebrospinal fluid (CFS), the least dense, appears black, and tissues and blood appear gray. In a CT scan, old damage appears darker because that region is filled with less dense CSF, whereas new damage appears lighter because blood is denser than CSF. Thus, a CT scan is a good method of examining loci of acquired brain damage. Developmental dyslexia (DD) is, by definition, not caused by brain damage. Thus, a CT scan is not the best way to study dyslexia.

Although a CT scan has been used to study abnormal brain organization in dyslexic brains, nowadays it is less used because of the availability of magnetic resonance imaging (MRI). Figure 2 demonstrates the normal pattern of hemispheric asymmetry, which shows the wider right frontal lobe and the wider left occipital lobe (Galaburda, LeMay, Kemper, \& Geschwind, 1978), whereas the typical abnormal asymmetry of adult dyslexic cases (Figure 3) demonstrates the reversed asymmetrical brain structure (Hier, LeMay, Rosenberger, \& Perlo, 1978). 


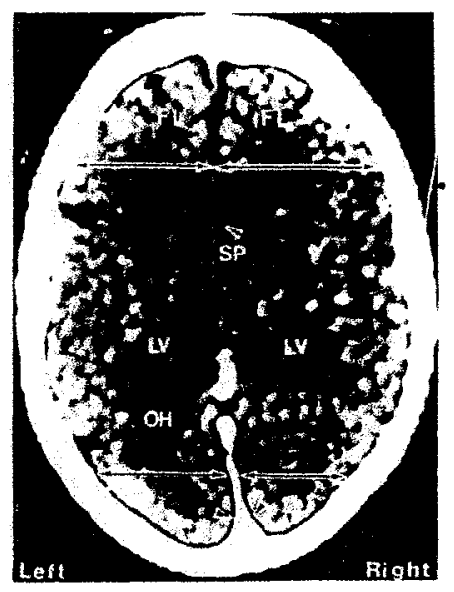

Figure 2. Computerized axial tomogram of a brain showing the usual pattern of hemispheric asymmetry. It is notable that the right frontal lobe (upper arrows) and the left occipital lobe (lower arrows) are wider. This tendency is more prominent in the left occipital horn $(\mathrm{OH})$ of the lateral ventricles $(\mathrm{LV})$. The septum pellucidum (SP) denotes the midline (Galaburda, LeMay, Kemper, \& Geschwind, 1978).

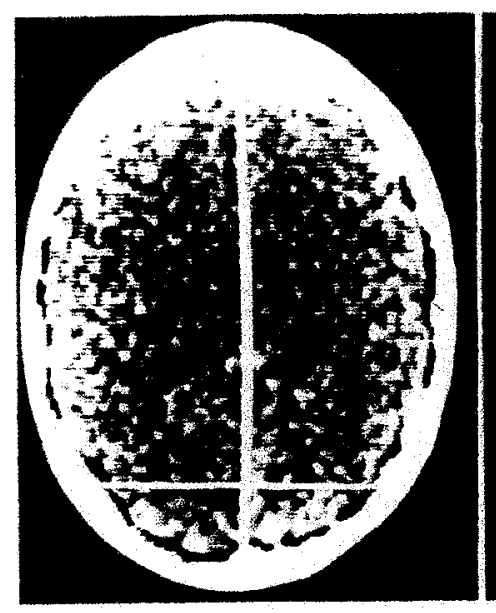

Common pattern (Left wider)

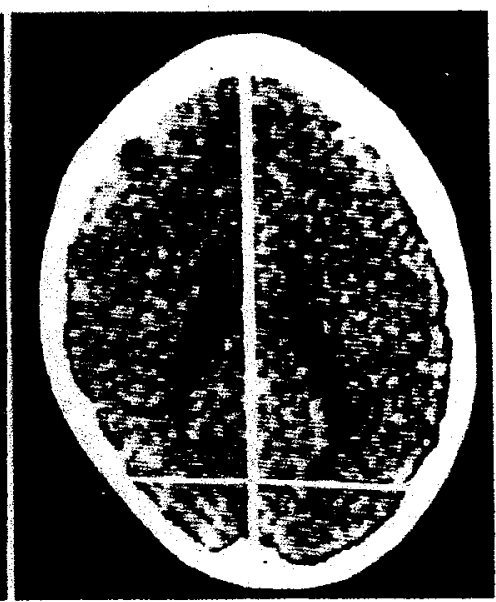

Reversed pattern

(Right wider)

Figure 3. Computerized brain tomograms of two dyslexic patients. Both transaxial sections are at the level of the ventricles. The heavy white strips lay vertically along the interhemispheric fissure; thin white strips lay horizontally across the posterior parietooccipital region. The brain at left shows the common pattern of cerebral asymmetry with the wider left parietooccipital region. Brain at right shows a marked reversal of usual cerebral asymmetry with a wider right parietooccipital region (Hier, LeMay, Rosenberger, \& Perlo, 1978). 


\section{Appendix C}

\section{Magnetic Resonance Imaging (MRI)}

This noninvasive technique uses a combination of radio waves and a strong magnetic field to detect the distribution of water molecules in living tissue (the hydrogen atoms in the water 'resonate' due to the combined effect of the radio waves and magnetic field). In this way, a computer can calculate brain tissue densities very accurately and generate a fine pictorial image (Figure 4). Thus, this technique can show a more precise and detailed brain image than a CT scan. A midsagittal section of MRI image (see Figure 5A) can show the position of the corpus callosum in the living brain, a large white matter tract critical to the present thesis. The diagnostic advantage of MRI over CT is shown in horizontal sections through the cerebral cortex (see Figure 5B). The MRI image can show more visible degeneration of white matter (myelinated axons which make synaptic contacts in different brain regions) of a patient with multiple sclerosis than a CT scan.

The MRI scan is also an excellent technique for studying the structure of the corpus callosum, because of its sensitivity to the fatty myelin sheaths. Since the corpus callosum is the main fiber bundle connecting the two cerebral hemispheres in the brain, the MRI is a good tool to study the interhemispheric connectivity associated with dyslexic brains. For example, a MRI study showed that the adult dyslexics had a smaller genu (anterior region of the corpus callosum) but no difference in the splenium (the posterior region of the corpus callosum) to the general population (Hynd, Hall, Novey, et al., 1995). An MRI, however, cannot provide a brain image at the neural cellular level. Thus, it is limited to study of the neuronal abnormalities of dyslexic brains (Galaburda \& Kemper, 1979). Since MRI 

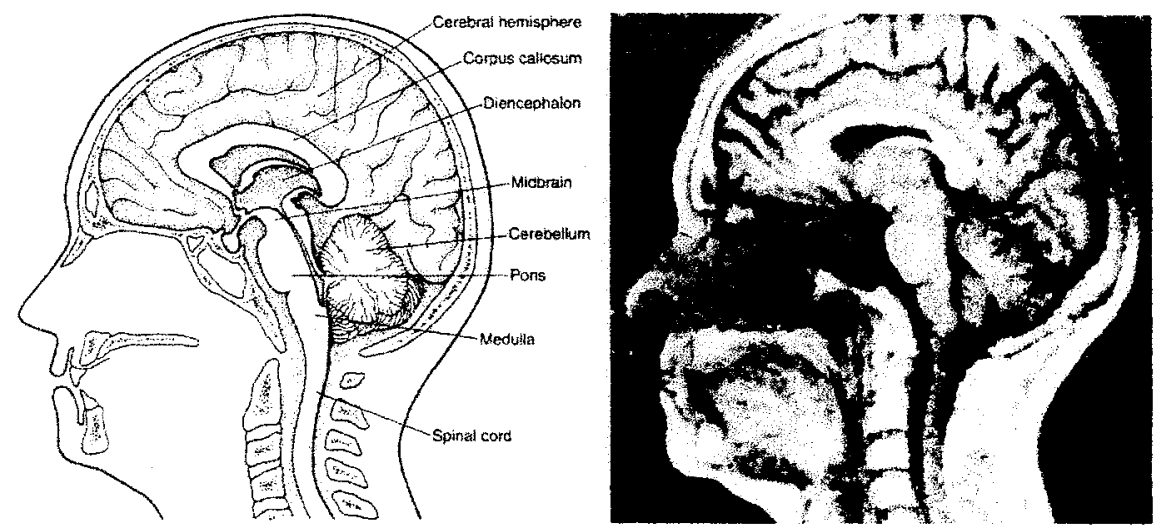

Figure 4. The MRI of a midsagittal section (cutting the brain between the two hemispheres down the midline) through the cerebral hemispheres, corpus callosum and spinal cord shows the living brain. The corpus callosum is a large fiber bundle that interconnects the left and right hemispheres. The schematic midsagittal section shows the position of the major brain structures (Kandel, Schwartz, and Jessell, 1991).

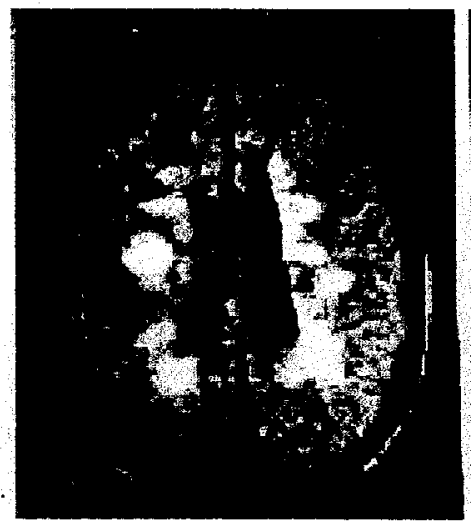

MRI

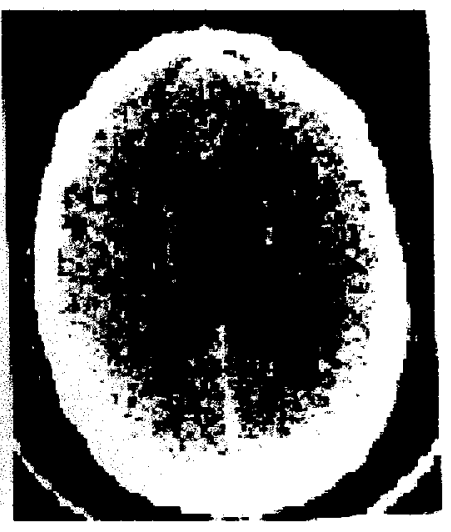

CT scan

Figure 5. (A): A midsagittal section shows the position of the corpus callosum in the magnetic resonance image of the living brain. (B): The diagnostic advantage of MRI over CT is shown in horizontal sections through the cerebral cortex and underlying white matter of a patient with multiple sclerosis. Demyelination of white matter is more clearly visible in the MRI scan (Kandel, Schwarts, and Jessell, 1991). 


\section{Appendix D}

\section{Positron Emission Tomography (PET)}

A PET scan, like the CT, uses radioisotopes to obtain an image of brain function. In the PET scan, however, the radiation is emitted by a substance introduced into the body rather than by radiation passing through the body (Banich, 1997). While engaging the subject in a specific cognitive task, we can measure local change of blood flow related to the task, such as reading or hearing. Figures 7 and 8 show a series of images with the PET scan that used F-labeled deoxyglucose while engaging in visual or auditory tasks. Red represents the highest metabolic rate. Gray matter, which contains the cell bodies and dendrites of neurons as well as the regions of synaptic contact, is metabolically more active than white matter, which contains the myelinated axons (Martin, Brust, \& Hilal, 1991).

One of the main disadvantages of PET is that a limited number of pictures (i.e., five or six pictures) can be taken from an individual per year because of the use of radiation. The other disadvantage is that the time required to obtain a picture of brain activity is approximately an hour. This time lapse is problematic because the PET method prevents the use of most cognitive tasks, such as naming, recognition, or judgement tasks, which measure quick response time. However, this method is suitable to measure ongoing cognitive tasks, such as reading, listening or seeing (Figures 7 and 8). 
Visual Stimulation

$$
\text { Eyes closed White light Complex scene }
$$

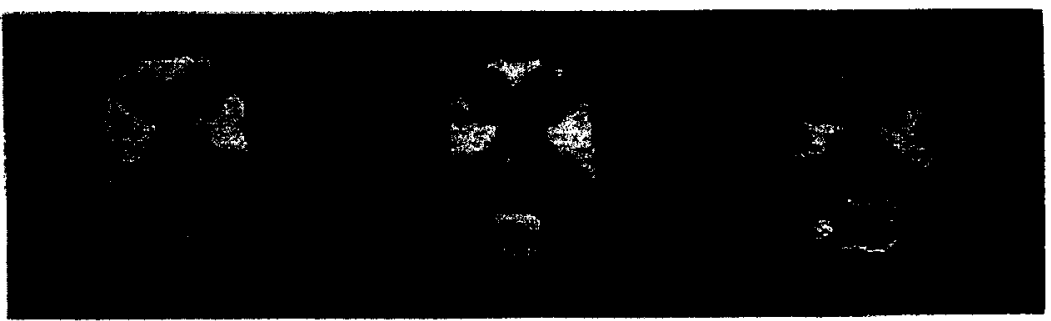

Figure 7. These PET scans show that different brain regions are activated by visual stimuli of different complexity. We can see the different level of activities in the primary visual cortex (area 17) by comparing the activities when the eye is closed (left), seeing white light (center) and a complex scene (right) (Courtesy of Drs. Michael E. Phelps and John C. Mazziotta: Kandel. Schwarts, and Jessell, 1991).

\section{Auditory Stimulation}

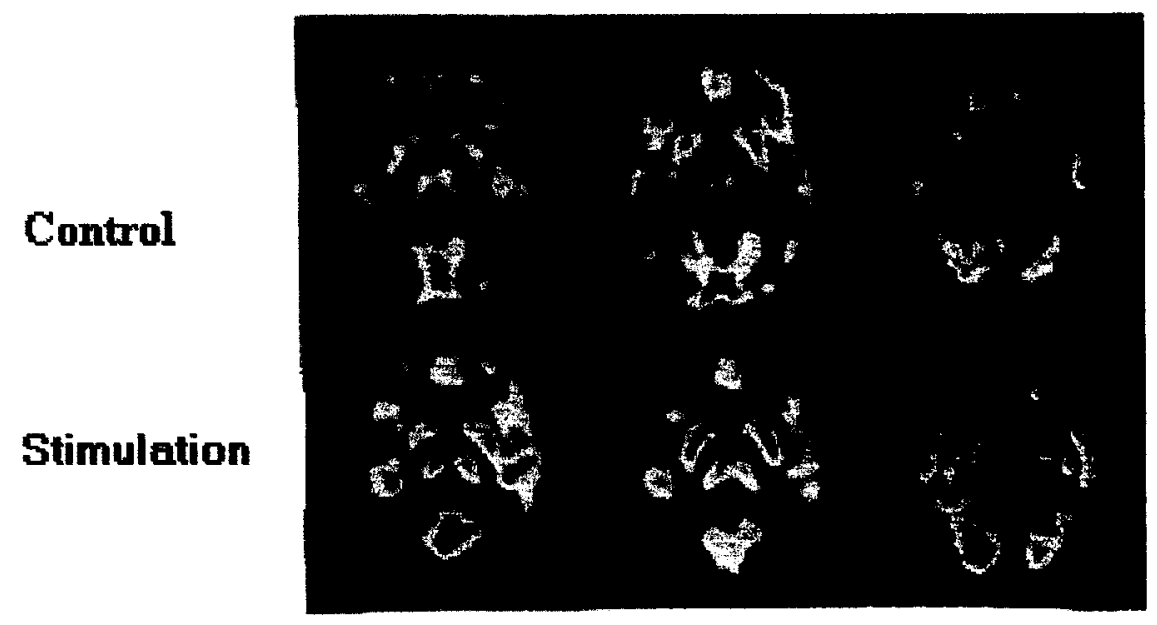

Figure 8. These PET scans show how auditory stimulation alters the pattern of activity at three different levels of the brain. The bottom images are from an experimental subject who was read a story and told to remember specific phrases. The top images are from a control subject who was not read the story. Listening to the story increases metabolic activity in the experimental subject's primary and higher-order auditory cortices (arrow, bottom left scan), as well as in the hippocampus (arrow, bottom right scan), a structure important for memory, (Courtesy of Drs. Michael E. Phelps and John C. Mazziotta: Kandel. Schwarts, and Jessell, 1991). 


\section{Appendix E}

\section{Functional Magnetic Resonance Imaging (fMRI)}

This is a variation of the MRI. In contrast to MRI, $\mathrm{MRI}$ provides functional information on the working of the brain. Brain activity is reflected as local changes in cerebral blood flow $(\mathrm{CBF})$ and blood oxygenation. Thus, $\mathrm{AMRI}$ can examine brain metabolism by using a contrast agent with magnetic properties, such as gadolinium, to measure blood flow. When a particular area of the brain is actively involved in a specific cognitive task, the local increase in oxygen rich blood is greater than in other areas which are not involved in the cognitive task (Figure 6).

Thus, unlike a PET scan, fMRI allows us to study brain activities using most cognitive tasks such as naming or recognition tasks. It is a promising method for the study of brain function, especially because the data supplied are comparable to those already obtained by cognitive tasks. A current problem is lack of availability of access to fMRI machines in research purposes.

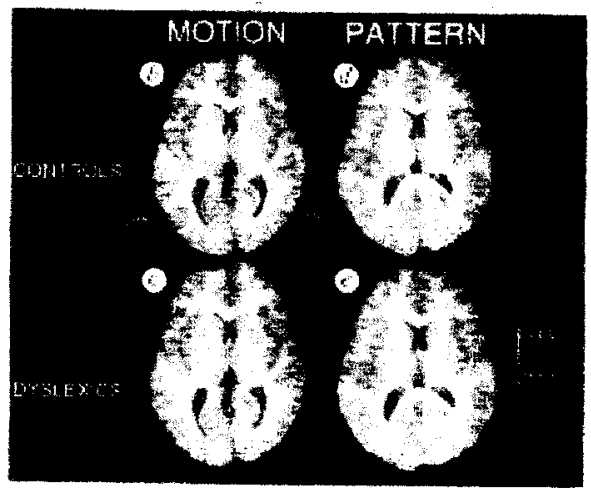

Figure 6. $\mathrm{fMRI}$ images show that dyslexics demonstrate less brain activation to a moving stimulus compared to their controls $(b, c)$, but do demonstrate similar activation to the pattern stimulus as their controls $(\mathrm{d}, \mathrm{e})$ (Eden, et al., 1996). 


\section{Appendix F}

Test of Word Reading Efficiency (TOWRE)

(Torgesen, et al., 1999)

Sight Word Efficiency (SWE)

$\begin{array}{llll}\text { is } & \text { work } & \text { crowd } & \text { uniform } \\ \text { up } & \text { jump } & \text { better } & \text { necessary } \\ \text { cat } & \text { part } & \text { inside } & \text { problems } \\ \text { red } & \text { fast } & \text { plane } & \text { absentee } \\ \text { me } & \text { fine } & \text { pretty } & \text { advertise } \\ \text { to } & \text { milk } & \text { famous } & \text { pleasant } \\ \text { no } & \text { back } & \text { children } & \text { property } \\ \text { we } & \text { lost } & \text { without } & \text { distress } \\ \text { he } & \text { find } & \text { finally } & \text { information } \\ \text { the } & \text { paper } & \text { strange } & \text { recession } \\ \text { and } & \text { open } & \text { budget } & \text { understand } \\ \text { yes } & \text { kind } & \text { repress } & \text { emphasis } \\ \text { of } & \text { able } & \text { contain } & \text { confident } \\ \text { him } & \text { shoes } & \text { justice } & \text { intuition } \\ \text { as } & \text { money } & \text { morning } & \text { boisterous } \\ \text { book } & \text { great } & \text { resolve } & \text { plausible } \\ \text { was } & \text { father } & \text { describe } & \text { courageous } \\ \text { help } & \text { river } & \text { garment } & \text { alienate } \\ \text { then } & \text { space } & \text { business } & \text { extinguish } \\ \text { time } & \text { short } & \text { qualify } & \text { prairie } \\ \text { wood } & \text { left } & \text { potent } & \text { limousine } \\ \text { let } & \text { people } & \text { collapse } & \text { valentine } \\ \text { men } & \text { almost } & \text { elements } & \text { detective } \\ \text { baby } & \text { waves } & \text { pioneer } & \text { recently } \\ \text { new } & \text { child } & \text { remember } & \text { instruction } \\ \text { stop } & \text { strong } & \text { dangerous } & \text { transient }\end{array}$


Phonetic Decoding Efficiency (PDE)

$\begin{array}{lll}\text { ip } & \text { barp } & \text { cratty } \\ \text { ga } & \text { stip } & \text { trober } \\ \text { ko } & \text { plin } & \text { deoate } \\ \text { ta } & \text { frip } & \text { glant } \\ \text { om } & \text { poth } & \text { sploosh } \\ \text { ig } & \text { vasp } & \text { dreker } \\ \text { ni } & \text { neest } & \text { ritlun } \\ \text { pim } & \text { shlee } & \text { hedfert } \\ \text { wum } & \text { guddy } & \text { bremick } \\ \text { lat } & \text { skree } & \text { nifpate } \\ \text { baf } & \text { felly } & \text { brinbert } \\ \text { din } & \text { clirt } & \text { clabom } \\ \text { nup } & \text { sline } & \text { drepnort } \\ \text { fet } & \text { dreef } & \text { shratterd } \\ \text { bave } & \text { prain } & \text { plofent } \\ \text { pate } & \text { zint } & \text { smuncrit } \\ \text { herm } & \text { bloot } & \text { pelnador } \\ \text { dess } & \text { trisk } & \text { fornalask } \\ \text { chur } & \text { kelm } & \text { fermabalt } \\ \text { knap } & \text { stone } & \text { crenidmoke } \\ \text { tive } & \text { lunaf } & \text { emulbatate }\end{array}$




\section{Appendix G}

Non-standardized Vocabulary List (Castles \& Coltheart, 1993)

\begin{tabular}{|c|c|c|c|}
\hline$\#$ & Regular & Irregular & Non-words \\
\hline 1 & take & come & gop \\
\hline 2 & free & sure & teg \\
\hline 3 & market & island & nad \\
\hline 4 & effort & answer & lif \\
\hline 5 & plant & blood & sut \\
\hline 6 & middle & pretty & sted \\
\hline 7 & check & break & mulp \\
\hline 8 & drop & lose & prin \\
\hline 9 & luck & soul & nint \\
\hline 10 & navy & iron & gren \\
\hline 11 & chicken & colonel & thim \\
\hline 12 & context & routine & chut \\
\hline 13 & wedding & ceiling & sith \\
\hline 14 & smog & quay & phot \\
\hline 15 & tail & bowl & giph \\
\hline 16 & victor & regime & hoil \\
\hline 17 & weasel & meringue & toud \\
\hline 18 & mist & shoe & gead \\
\hline 19 & infest & indict & doil \\
\hline 20 & curb & pint & roin \\
\hline 21 & nerve & gauge & gurdet \\
\hline 22 & pump & tomb & torlep \\
\hline 23 & peril & choir & tadlen \\
\hline 24 & radish & debris & latsar \\
\hline 25 & brandy & cough & polmex \\
\hline 26 & stench & brooch & tashet \\
\hline 27 & ditty & beret & sothep \\
\hline 28 & marsh & yacht & miphic \\
\hline 29 & flannel & bouquet & lishon \\
\hline 30 & cord & wolf & dethix \\
\hline
\end{tabular}


Appendix 16

\section{Appendix $\mathrm{H}$}

Example of the Test of Non-Verbal Intelligence-3 (TONI-3)

(Brown, et al., 1980)
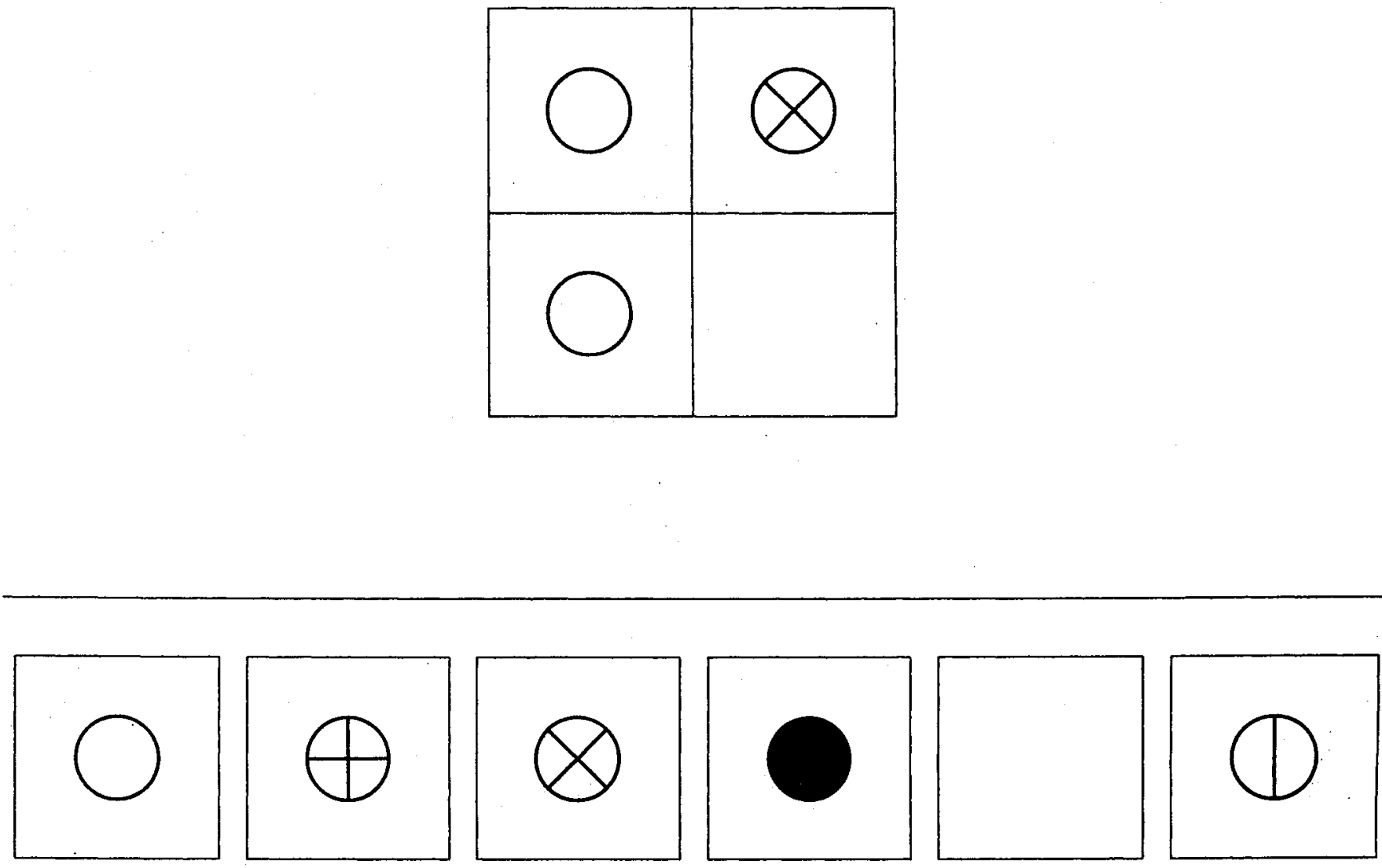


\section{Appendix I}

\section{Parent Questionnaire}

Winter, 2002

\section{Please mark with an $\mathrm{X}$ or $\checkmark$.}

1. Please select the number that you think best describes your child's educational opportunities.

$\begin{array}{lllll}\text { very poor } & \text { poor } & \text { average } & \text { good } & \text { very good } \\ 1 \ldots \ldots & 2 \ldots \ldots & 3 \ldots \ldots & 4 \ldots \ldots & 5 \ldots \ldots\end{array}$

2. Please select the number that you think best describes your child's reading skills.

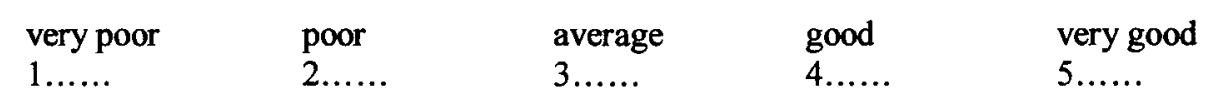

3. Please select the number that you think best describes your child's academic achievement in math and science.

Math

$\begin{array}{lllll}\text { very poor } & \text { poor } & \text { average } & \text { good } & \text { very good } \\ 1 \ldots \ldots & 2 \ldots \ldots & 3 \ldots \ldots & 4 \ldots \ldots & 5 \ldots \ldots \ldots\end{array}$

Science
$1 \ldots \ldots$
$2 \ldots \ldots$
$3 \ldots \ldots$
$4 \ldots .$.
5.......

4. Has your child experienced a head injury?

$\square$ Yes

If Yes, When was it?

No

5. Has your child been diagnosed with Attentional Deficit Disorder with or without impulsivity? (i.e., $\mathrm{ADHD}$ or $\mathrm{ADD}$ )

$\square$ Yes

$\square$ No

6. Has your child been diagnosed with a learning disability by a qualified professional (e.g., psychologist or medical professional)?
Yes
If Yes, please identify
(e.g., math, reading)

No

7. Does your child have a medical or psychiatric problem, which might influence the outcome of this study (e.g., hearing, visual or emotional problem)?

$\square$ Yes

If Yes, please identify

No 


\section{Carleton}

UN IVER S I Y

Canada's Capital University
Department of Psychology

Faculty of Arts and Social Sciences

B550 Loeb Building

1125 Colonel By Drive

Ottawa, ON K1S 5B6 Canada

Tel: (613) 520.2644

Fax: (613) 520-3667

\section{LETTER TO PARENTS}

Fall 2001

Dear parent(s) or guardian(s),

Researchers from Carleton University are conducting a project concerned with improving our understanding of how our brain functions during reading. The Carleton University Research Ethics Committee for Psychological Research as well as the Ottawa-Carleton Research Advisory Committee and the principal of the school have granted permission for us to request your cooperation in this study.

We are seeking male students from grades 9 to 12 who speak English as their mother tongue for our project. Your child's participation will be of great help for those who suffer from severe reading problems. In addition, your child will experience science in a hands-on way. We hope that the participation may stimulate your child's scientific interests in different ways from classroom study. Each participant will be offered a choice of a small token of appreciation or $\$ 20$ for the participation.

Participation is completely voluntary, and your child may choose to withdraw at any point during the session. Your child will be entitled to receive a gift or $\$ 20$ even if $\mathrm{s} / \mathrm{he}$ decides to withdraw. Participation by your child will be kept strictly confidential. The information collected will be coded such that your child's name is not associated with the data. All information will be seen only by the researchers involved in this study, and will be used solely for research purposes. The attached questionnaire pertains to your child's health, academic performance (science \& math), and educational history. This information is necessary to allow us to compare students and to better understand their reading ability.

If you and your child would like to participate, please complete the enclosed Consent Form and Questionnaire and mail it using the prepaid envelope or contact me at 235-7283. If you or your child would like to know more about the study, please contact me at 325-0422.

Sincerely,

Hiroko Sotozaki, M.A.

Ph.D. Candidate in Psychology 


\section{BRIEF SUMMARY OF THE STUDY}

Although most children learn to read with minimum assistance, some children experience problems in learning to read. Some children with reading problems specifically show their problems in learning phonetic rules for reading words (e.g., hat and mat, bike and kite, station and inspection). We do not know exactly what brain mechanisms are involved in reading acquisition and how they may interact with one another. A human brain has two sides, left and right. It is believed that the left side brain plays a special role in the processing of spoken and written language in most people. Recent studies, however, suggest that both sides of the brain may be required for reading. We hypothesize that the timing and quality of communication between the two sides of the brain are critical in reading. The purpose of this project is to test this hypothesis. We are asking you to permit your child to participate in our study. We are also asking parents to complete several brief questionnaires.

There are three tasks involved in this study: (1) A finger localization task, (2) A rhyming judgement task, and (3) A primed lexical decision task.

In the first task, a researcher will lightly touch from two to four fingers on your child's hand in sequence. Your child will be asked to repeat the finger sequence using the thumb. For the second task, your child will look at a computer screen, and will be shown a pair of words very briefly (e.g., bat/mat, eye/cow). He will be asked to indicate whether or not the words rhyme by clicking either 'Yes' or 'No' on a computer mouse. For the third task, again using a computer, your child will also see a pair of words (e.g., ham/egg), but this time some pairs are made of 'words' and 'nonsense words' (e.g., hat/zod). Using the mouse, your child will indicate whether or not the second word is a 'real word'. A researcher will ask your child some questions about his handedness and his reading habits. Your child will also be asked to complete a brief reading test, a vocabulary test, and a reasoning ability test.

The study takes s place at your child's school during class time and at the teacher and student's convenience. Every effort will be made to schedule testing so that your child will not miss any important classes. If you or your child wish, the testing can be scheduled after school. A researcher will meet each student individually. Participation requires approximately one hour and 30 minutes with a 5-minutes break in the middle of the testing. The tasks are straightforward, and participants usually find the activities interesting. There are no known risks associated with this study.

Should you have any questions or comments about this research, please contact:

- Ms. Hiroko Sotozaki (M.A.) (Principal Investigator, 520-2600, ext. 2654 or e-mail: hsotozak@ccs.carleton.ca

- Dr. S. Parlow (Faculty Sponsor in Dept of Psychology at Carleton University, 520-2600, ext. 2691)

Should you have any ethics concerns about this research, please contact:

- Dr. M. Gick (Chair, Carleton University Research Ethics Committee for Psychological Research, 5202600, ext. 2664)

Should you have any other concerns about this study, please contact:

- Dr. K. Matheson (Chair, Dept. of Psychology, 520-2600, ext. 2648)

You may also write to them at the Dept. of Psychology, Carleton University: 1125 Colonel By Dr. Ottawa, ON. K1S 5B6 


\section{Appendix J}

\section{Recruitment Announcement Published in a Monthly Bulletin of the Learning Disabilities Association-Ottawa}

\section{Recruitment for A Scientific Study}

Looking for participants for my $\mathrm{Ph}$. $\mathrm{D}$. thesis project. It focuses on how the brain functions while reading associated with reading disability. The participants include the following students:

(1) Diagnosed with LD in reading

(2) No significant problems in math/science

(3) Males aged between $14-18$

(4) English as mother tongue

The study involves three experimental tasks; to repeat a sequence of finger movements when lightly touched, to indicate whether or not letter strings on a computer screen is a word, and whether or not a presented word pair is rhymed. The tasks are simple and the words are easy. It takes about $70 \mathrm{~min}$. The participant will be offered a small gift or $\$ 20$ from their participation. Some tests (e.g., reading, vocabulary, reasoning ability, and handedness) will be required to complete. Ethical approval was granted for the study from Carleton University. If you are interested in the study, please contact Hiroko Sotozaki at (235) 7283/e-mail:ysotozak@magi.com. 


\section{Appendix K}

\section{Student Questionnaire}

Fall 2002

The experimenter will fill out the questions.

Participant \#:

Grade:

Age:

1. Please tell me the number that you think best describes how much you enjoy reading outside of school (e.g., Internet articles, novels, magazines, comics, newspapers, etc.), when 1 represents 'not at all' and 5 represents 'very much'.
not at all
average
very much
1......
$2 . .$.
3......
4
5.......

2. Please tell me the number that you think best describes how much time you spend reading everyday for any type of reading (e.g., Internet articles, novels, magazines, newspapers, etc.).

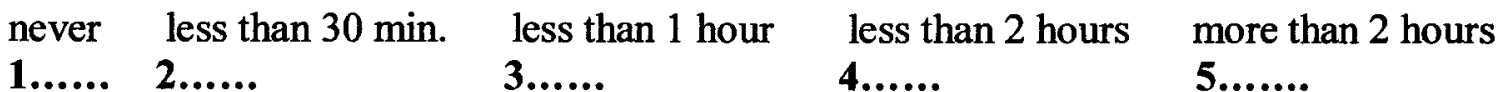




\section{Appendix L}

Edinburgh Handedness Inventory (Oldfield, 1971)

Please indicate your preference on the use of hands in the following activities by putting $a^{\prime \prime}++$ " in the appropriate column. Where the preference is so strong that you would never try to use the other hand unless absolutely forced to, put " ++ ". If in any case you are really indifferent, put " + " in both columns.

Some of the activities require both hands. In these cases, the part of the task, or object, for which hand preference is wanted, is indicated in brackets.

Please try to answer all the questions, and only leave a blank if you have no experience at all of the object or task.

\begin{tabular}{|l|l|l|}
\hline & \multicolumn{1}{|c|}{ LEFT } & \\
\hline 1. Writing & & \\
\hline 2. Drawing & & \\
\hline 3. Throwing & & \\
\hline 4. Scissors & & \\
\hline 5. Toothbrush & & \\
\hline 6. Knife (without fork) & & \\
\hline 7. Spoon & & \\
\hline 8. Broom (upper hand) & & \\
\hline 9. Striking a match (match) & & \\
\hline 10. Opening a box (lid) & & \\
\hline
\end{tabular}

1) Which foot do you prefer to kick with?

2) Which eye do you use when using only one?

\begin{tabular}{|l|l|}
\hline & \\
\hline & \\
\hline
\end{tabular}

\begin{tabular}{|l|l|l|l|}
\hline LQ & DECILE & \\
\hline
\end{tabular}




\section{Questions}

Since it was expected that DD students and young students might have difficulty answering some of the questions in the Edinburgh Handedness Inventory by themselves, the investigator asked the following questions prior to filling out the Inventory.

1. Writing: When you write a letter/note, which hand do you usually use?

(Right Left) (Always, Sometimes use the other hand, Never use the other hand)

2. Drawing: When you draw a picture, which hand do you usually use?

(Right Left) (Always, Sometimes use the other hand, Never use the other hand)

3. Throwing: When you throw a ball, which hand do you prefer to use?

(Right Left) (Always, Sometimes use the other hand, Never use the other hand)

4. Scissors: When you cut a paper with scissors, which hand do you use?

(Right Left) (Always, Sometimes use the other hand, Never use the other hand)

5. Toothbrush: When you clean your teeth, which hand do you use?

(Right Left) (Always, Sometimes use the other hand, Never use the other hand)

6. Knife without fork: When you eat meat at supper, in which hand do you hold a knife?

(Right Left) (Always, Sometimes use the other hand, Never use the other hand)

7. Spoon: When you eat soup, which hand do you prefer to use?

Right Left) (Always, Sometimes use the other hand, Never use the other hand)

8. Broom: When you clean up leaves with a broom, which way do you hold the broom?

(Right Left) (Always, Sometimes use the other hand, Never use the other hand)

9. Striking a match: When you have a birthday, which hand do you light the candle with?

(Right Left) (Always, Sometimes use the other hand, Never use the other hand)

10. Opening a box (lid): When you open a jar, which hand do you usually use?

(Right Left) (Always, Sometimes use the other hand, Never use the other hand) 


\section{Appendix M}

\section{Finger Localization Protocol}

For this task, I will lightly touch the tip of two, three or four of your fingers. Your task is to repeat the finger sequence immediately after I touch them and "go". You will show me the same finger sequence in the same order by touching the thumb on either the same hand or the opposite hand. Like this. Do you have any questions?

Let's practice now.

\section{Practice for Block 1 \& Block 2}

First Hand Stimulated: Right Hand Left Hand

Please place your (left or right) hand through this screen, palm down on this pillow. I would like you to repeat the finger sequence by touching the thumb on the same hand. Remember to wait until I say go before you repeat the finger sequence.

\section{$\underline{\text { Uncrossed }}$}

Practice 1: $2 \_$Practice 2: $1 \_2 \_$Practice 3: $1 \_2 \_3 \ldots$.

Now place both of your hands through the screen. I would like you to repeat the finger sequence by touching the thumb on the opposite hand.

\section{Crossed}

Practice 1: 2 Practice 2: $1 \_3 \_$Practice $1 \_2 \_3 \ldots$. 


\section{Block 1}

First Hand Stimulated:

Right Hand

Left Hand

Condition:

Crossed

Uncrossed

Now that you know how to do it, we'll start. For this first set of items, I will only touch your (left/right) hand. I want you to:

- Repeat the finger sequence by touching the thumb on the same hand. Please place your (left/right) hand through the screen.

- Repeat the finger sequence by touching the thumb on the opposite hand. Please place your both hands through the screen.

Trial 1: 3

Trial 2: 3 2

Trial 3: 2 4

Trial 4:

Trial 5: 3 3

Trial 6: $1{ }^{2}{ }^{2}+4$

Trial 7: 2

Trial 8: 3 1 4

Trial 9: 1 4

Trial 10: $2 \_3 \_2$

Trial 11: 4

Trial 12: 1 4

Trial 13: 3 2

Trial 14: 2 1 4

Trial 15:
Trial 16:

Trial 17: 2

Trial 18: $3 \_4+2$

Trial 19: $4{ }_{3}^{3} 3 \_1$

Trial 20: 2

Trial 21: $1 \_4+3 \_2$

Trial 22: $3{ }^{3} 3{ }^{3} \quad 2 \_4$.

Trial 23: $3 \_2$

Trial 24: $4 \quad 2 \quad 1 \quad 3$

Trial 25: 3

Trial 26: $2 \_4+1$

Trial 27: $2 \_3 \_4 \_1$

Trial 28: $1 \_2$

Trial 29: 3

Trial 30: 4 


\section{Appendix $\mathbf{N}$}

\section{Stimulus Lists for Experiment 2 (Rhyming Judgment Task)}

The four lists were counterbalanced across the conditions (Unilateral RVF, Bilateral RVF, Unilateral LVF, Bilateral LVF), and the order of stimuli was randomized in each condition per participant.

List 1

\begin{tabular}{ccc}
\hline$\#$ & Rhymed & Non-rhymed \\
\hline 1 & slip-ship & pond-four \\
2 & rule-tool & panda-tray \\
3 & pail-tale & mold-calm \\
4 & clear-cheer & bag-fox \\
5 & year-veer & gown-pass \\
6 & write-sight & time-port \\
7 & hare-heir & chalk-lord \\
8 & ran-van & fell-fall \\
9 & date-late & deaf-bead \\
10 & team-beam & loss-lost \\
11 & damp-camp & pain-pale \\
12 & rake-sake & wipe-wide \\
13 & jail-trail & food-fool \\
14 & pool-cool & stand-stunt \\
15 & belt-felt & male-maid
\end{tabular}

List 3

\begin{tabular}{ccc}
\hline & List 3 & Non-rhymed \\
\hline$\#$ & Rhymed & nest-boat \\
\hline 1 & come-drum & chat-gate \\
2 & pear-pair & kite-trip \\
3 & week-weak & pot-jar \\
4 & gear-beer & fake-dive \\
5 & whale-scale & door-yard \\
6 & might-quite & radio-goat \\
7 & here-hear & hell-hall \\
8 & gum-sum & tear-torn \\
9 & deal-meal & ripe-rope \\
$\mathbf{1 0}$ & gate-hate & deed-dine \\
$\mathbf{1 1}$ & bone-tone & whip-witch \\
$\mathbf{1 2}$ & bake-take & wine-wind \\
$\mathbf{1 3}$ & rock-dock & choir-chair \\
$\mathbf{1 4}$ & slum-some & side-site \\
15 & thing-sting &
\end{tabular}

List 2

\begin{tabular}{cc}
\hline Rhymed & Non-rhymed \\
\hline root-cute & cake-rain \\
plum-dumb & send-drug \\
dear-deer & rose-cave \\
tan-can & son-hen \\
wear-ware & stone-store \\
tight-bite & army-nose \\
their-there & cloud-jeep \\
lip-hip & mail-mall \\
pen-ten & tail-tall \\
heat-beat & fear-bear \\
lump-hump & give-gift \\
clip-flip & top-tap \\
weep-beep & heap-heel \\
trunk-junk & poem-poet \\
sat-pat & boss-toss
\end{tabular}

List 4

\begin{tabular}{cc}
\hline List 4 & Non-rhymed \\
\hline Rhymed & firm-path \\
\hline mite-night & bent-ache \\
sail-male & trap-walk \\
monk-punk & key-mug \\
rear-mere & chain-stop \\
brake-break & plant-glue \\
spite-fright & sheep-coal \\
cling-spring & push-pull \\
tin-pin & wash-cash \\
rare-fare & love-cove \\
grip-drip & snake-snail \\
sell-tell & type-tape \\
dare-bare & lent-lend \\
make-wake & well-wall \\
mum-rum & grade-grand \\
ring-bring &
\end{tabular}

Note: the highlighted words are orthographically similar pairs in both types of pairs. 


\section{Appendix 0}

\section{A Pilot Study: Test-Retest Reliability}

This analysis was done for a test of reliability of the visual half-field presentation procedure used for the present thesis project.

\section{Methods}

\section{Participants}

One 12.09 year-old girl with average academic achievements, within a normal range of non-verbal intelligence (TONI-3 $=93$ ), and age equivalent reading ability (TOWRE $=$ 118) was tested twice in a 10-day interval in a rhyming judgement task using the visual half-field presentation paradigm.

\section{Apparatus}

The same apparatus described in Chapter11 was used to present all stimuli.

\section{Stimulus materials}

The stimuli lists were reproduced in Appendix N: 15 rhymed (root-cute) and 15 non-rhymed (cake-rain). They were equated in terms of word length, word frequency to the second word of a pair. The order of the four lists was different in Test 1 and Test 2 . The stimuli were randomized for both the tests by computer.

\section{Procedure}

The visual half-field presentation procedure was identical to that described in the Procedure section in Chapter 11. 


\section{Analysis}

A 2 (Test: first Test, second Test) $\times 2$ (Condition: unilateral, bilateral) $\times 2$ (Visual Field: right, left) ANOVA with a mixed repeated measures analysis was performed on the data. Condition and Visual Field were within factors. Test was treated as a between factor. Reaction time was assessed as a dependent variable. Because this analysis involved one subject, accuracy was coded either 1 for a correct or 0 for an incorrect response. Thus, the correct percentage was unable to enter the analysis as a dependent variable.

\section{Results}

Overall mean accuracy was $84.17 \%$ for the first Test and $86.67 \%$ for the second Test. There was a main effect of Condition, $F(1,46)=13.8, p<.001, \mathrm{y}^{2}=.231$. That is, the stimuli presented in the Bilateral Condition $(M=864.52 \mathrm{msec}, S D=200.46)$ were responded to more slowly than those presented in the Unilateral Condition $(M=784.967$ msec, $S D=139.30)$ across the visual fields. This suggests that this participant did not demonstrate an advantage of bilateral presentation. The lack of main effect and the interaction of Visual Field indicates the absence of the RVF superiority effect. More importantly, however, no main effect and the interaction of the Test variable, $F(1,46)=$ $2.37, p=.131, \mathrm{n}^{2}=.049$, indicates that the two tests did not significantly differ from each other.

\section{Discussion}

This pilot study was conducted to test the reliability of the visual half-field presentation procedure. The results showed that there was no significant difference between 
the first and second tests. This demonstrated the reliability of the present visual half-field presentation procedure. The data are summarized in Figure 1.

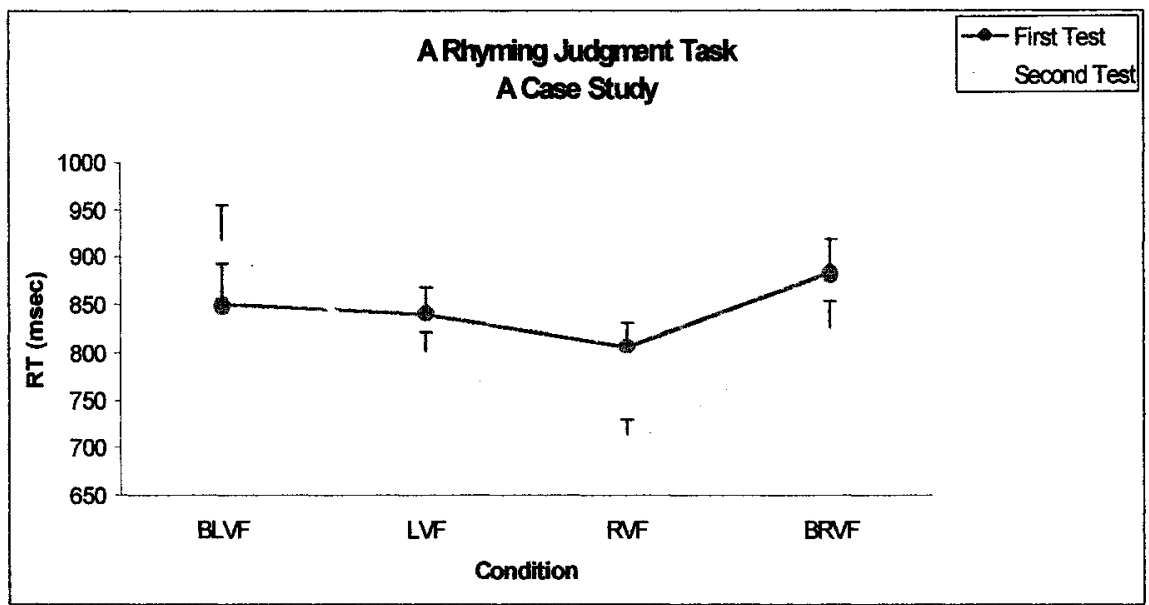

Figure 1. The test-retest reliability for the visual half-field presentation. 


\section{Appendix P}

\section{Stimulus Lists for Experiment 3 (Lexical Decision Task)}

The four lists were counterbalanced across the conditions (Unilateral RVF, Bilateral RVF, Unilateral LVF, Bilateral LVF), and the order of stimuli was randomized in each condition per participant.

\begin{tabular}{|c|c|c|c|c|c|c|}
\hline$\#$ & $\begin{array}{l}\text { List } 1 \\
\text { Associated }\end{array}$ & $\begin{array}{l}\text { Non- } \\
\text { Associated }\end{array}$ & Word-Nonword & $\begin{array}{l}\text { List } 2 \\
\text { Associated }\end{array}$ & $\begin{array}{l}\text { Non- } \\
\text { Associated }\end{array}$ & $\begin{array}{l}\text { Word- } \\
\text { Nonword }\end{array}$ \\
\hline $\begin{array}{c}1 \\
2 \\
3 \\
4 \\
5 \\
6 \\
7 \\
8 \\
9 \\
10 \\
11 \\
12 \\
13 \\
14 \\
15\end{array}$ & $\begin{array}{l}\text { slow-fast } \\
\text { hard-soft } \\
\text { city-town } \\
\text { green-grass } \\
\text { ocean-sea } \\
\text { square-circle } \\
\text { earth-ground } \\
\text { red-black } \\
\text { chair-sit } \\
\text { song-sing } \\
\text { book-read } \\
\text { key-lock } \\
\text { lemon-sour } \\
\text { skip-rope } \\
\text { cheese-mouse }\end{array}$ & $\begin{array}{l}\text { term-dear } \\
\text { club-busy } \\
\text { pond-plan } \\
\text { snail-chest } \\
\text { spade-kid } \\
\text { potato-elbow } \\
\text { scale-sister } \\
\text { lot-stock } \\
\text { table-add } \\
\text { open-save } \\
\text { boot-lose } \\
\text { ice-boss } \\
\text { model-hint } \\
\text { span-coin } \\
\text { weight-brand }\end{array}$ & $\begin{array}{l}\text { west-foin } \\
\text { memo-nusy } \\
\text { slope-tavor } \\
\text { style-noot } \\
\text { lunch-zea } \\
\text { dollar-dircle } \\
\text { digit-sust } \\
\text { ant-bindow } \\
\text { diary-dit } \\
\text { walk-dand } \\
\text { dial-gead } \\
\text { toy-mook } \\
\text { dream-frass } \\
\text { yard-verm } \\
\text { digest-trand }\end{array}$ & $\begin{array}{l}\text { boy-girl } \\
\text { eagle-bird } \\
\text { smooth-rough } \\
\text { mutton-sheep } \\
\text { house-home } \\
\text { cold-warm } \\
\text { moon-sun } \\
\text { street-road } \\
\text { river-lake } \\
\text { sky-blue } \\
\text { carpet-floor } \\
\text { hammer-nail } \\
\text { ugly-pretty } \\
\text { star-bright } \\
\text { doctor-sick }\end{array}$ & $\begin{array}{l}\text { ash-face } \\
\text { role-seed } \\
\text { sample-funny } \\
\text { string-brick } \\
\text { voice-rate } \\
\text { mark-calm } \\
\text { milk-bar } \\
\text { bullet-wife } \\
\text { brain-card } \\
\text { mop-note } \\
\text { parent-trial } \\
\text { dancer-rock } \\
\text { glad-visual } \\
\text { cube-middle } \\
\text { figure-safe }\end{array}$ & $\begin{array}{l}\text { hen-gope } \\
\text { bass-zird } \\
\text { couple-vough } \\
\text { corner-nabel } \\
\text { strap-dest } \\
\text { iron-darm } \\
\text { lady-tas } \\
\text { wallet-zoad } \\
\text { class-pake } \\
\text { bug-fote } \\
\text { pillow-tolor } \\
\text { engine-wens } \\
\text { wolf-kretty } \\
\text { soap-dright } \\
\text { tomato-zick }\end{array}$ \\
\hline
\end{tabular}

List 3

\begin{tabular}{cl} 
\# & Associated \\
\hline 1 & king-queen \\
2 & lamp-light \\
3 & bath-clean \\
4 & thief-steal \\
5 & afraid-scare \\
6 & noise-loud \\
7 & child-adult \\
8 & bible-church \\
9 & deep-water \\
10 & cat-dog \\
11 & arm-leg \\
12 & sugar-sweet \\
13 & hop-jump \\
14 & stove-heat \\
15 & train-track
\end{tabular}

\begin{tabular}{ll}
$\begin{array}{l}\text { Non- } \\
\text { Associated }\end{array}$ & Word-Nonword \\
\hline golf-cover & port-gover \\
poem-empty & atom-diger \\
pave-grade & heal-skade \\
proof-crowd & stack-groof \\
current-drunk & orient-prunk \\
clerk-well & sword-kell \\
draft-grain & tower-vrain \\
orbit-member & elite-pember \\
fuel-point & aunt-foint \\
fee-gun & age-zog \\
era-aid & cop-teg \\
honey-trend & phone-vock \\
fan-chip & mad-sape \\
porch-grin & fence-trin \\
match-stair & shift-drack
\end{tabular}

List 4

\begin{tabular}{|c|c|c|}
\hline Associated & $\begin{array}{l}\text { Non- } \\
\text { Associated }\end{array}$ & $\begin{array}{l}\text { Word- } \\
\text { Nonword }\end{array}$ \\
\hline $\begin{array}{l}\text { short-long } \\
\text { bread-butter } \\
\text { spider-web } \\
\text { lion-tiger } \\
\text { foot-hand } \\
\text { joy-happy } \\
\text { baby-cry } \\
\text { head-hair } \\
\text { yellow-color } \\
\text { bed-sleep } \\
\text { number-one } \\
\text { needle-sharp } \\
\text { stem-flower } \\
\text { apple-fruit } \\
\text { shoe-sock }\end{array}$ & $\begin{array}{l}\text { urban-free } \\
\text { pilot-parade } \\
\text { screen-rat } \\
\text { salt-witch } \\
\text { neck-work } \\
\text { box-north } \\
\text { wind-guy } \\
\text { pool-tree } \\
\text { forest-plate } \\
\text { jet-paint } \\
\text { summer-oil } \\
\text { winter-worth } \\
\text { east-coffee } \\
\text { plane-crime } \\
\text { wing-trap }\end{array}$ & $\begin{array}{l}\text { spare-jong } \\
\text { clock-garade } \\
\text { prince-zat } \\
\text { wire-fitch } \\
\text { arch-dife } \\
\text { cab-dappy } \\
\text { echo-hin } \\
\text { pole-kree } \\
\text { window-vlate } \\
\text { pin-laint } \\
\text { second-ket } \\
\text { pencil-lorth } \\
\text { sand-poffee } \\
\text { bride-trime } \\
\text { coal-skap }\end{array}$ \\
\hline
\end{tabular}




\section{Appendix Q}

\section{A Pilot Study: Stimulus Lists Used in Experiment 3}

This pilot study was conducted to examine if the four stimulus lists developed for a primed lexical decision task (Experiment 3) produced priming effects in normal young adults.

\section{Methods}

\section{Participants}

Twenty right-handed university students (mean age of 21.8 years, $S D=3.54,12$ females and 8males), whose average non-verbal IQ (TONI-3) was $102(S D=10.36)$ and average standardized reading score (TOWRE) was $105(S D=16.06)$, participated in the pilot study as part of an introductory psychology course credit.

\section{Apparatus}

The same apparatus described in Chapter 12 was used to present all stimuli.

\section{Stimulus materials}

The stimuli pairs reproduced in Appendix P were used: 15 associated (cat-dog), 15 non-associated (pen-bus), and 15 non-word (toy-dit). They were equated in terms of word length and word frequency (Francis \& Kucera, 1982) to target and the strength of association (Postman \& Keppel, 1970) between the associated pairs. The order of the four lists was counterbalanced across the conditions (Unilateral LVF, Bilateral LVF, Unilateral RVF and Bilateral RVF). That is, when List 1 was used for the Unilateral LVF Condition for the first participant, List 1 was used for the Bilateral LVF Condition for the second 
participant, and so fourth. In addition, the Condition was also counterbalanced. That is, when the first participant started with the Unilateral LVF Condition, the second participant started with the Bilateral LVF Condition, and so forth. Thus, each list and condition had an equal chance of being be placed in any order within the four conditions and in any order within the four lists. The stimuli in each list were randomized by computer for each participant.

\section{Procedure}

The visual half-field presentation procedure was identical to that described in the Procedure section in Chapter 11.

Analysis

A 2 (Condition: unilateral, bilateral) x 2 (Visual Field: right, left) $\times 3$ (Stimulus Pair: associated, non-associated, non-word) MANOVA with a repeated measures was performed on the data. Condition, Visual Field and Stimulus Pairs were within subject factors. Two dependent variables were reaction time and percentage correct to the target.

\section{Results}

Overall mean accuracy was $81.1 \%$ correct across the word pairs (i.e., associated and non-associated pairs).

There were two main effects: Stimulus Pair, $F(2,16)=18.63, p<.000, \mathrm{y}^{2}=.823$; and Visual Field, $F(2,18)=7.06, p<.005, \mathrm{n}^{2}=.440$. The pairwise comparisons revealed that the associated pairs $(M=786.05 \mathrm{msec}, S D=226.25)$ were responded to faster than the non-associated pairs $(M=833.57 \mathrm{msec}, S D=235.25), p=.004$, and more accurately $(M=$ $86.17 \%, S D=12.03)$ than the non-associated pairs $(M=80.83 \%, S D=10.50), p=.000$. In 
addition, the non-word pairs $(M=899.18 \mathrm{msec}, S D=200.75)$ were responded to more slowly than the non-associated pairs across the groups, $p=.007$. Regarding the main effect on Visual Field, the pairwise comparisons revealed that the target presented in the RVF ( $M$ $=815.39 \mathrm{msec}, S D=203.33)$, was responded to faster $p<.029$, and more accurately $(M=$ $84.56 \%, S D=11.50), p<.005$, than that that presented in the LVF $(M=863.0 \mathrm{msec}, S D=$ 238.16; $M=77.45 \%, S D=13.91$ ). The effect of Condition was not significant, indicating that there was no difference between the Unilateral and Bilateral Conditions. The data are summarized in Table 1.

Table 1

Reaction Time and Percentage of Correct Responses

\begin{tabular}{|c|c|c|c|c|c|c|c|c|}
\hline & & RT & (msec) & & & Correct & $(\%)$ & \\
\hline Stimulus type & $\begin{array}{l}\text { LVF } \\
(S D)\end{array}$ & $\begin{array}{c}\text { BLVF } \\
(S D)\end{array}$ & $\begin{array}{l}\text { RVF } \\
(S D) \\
\end{array}$ & $\begin{array}{c}\text { BRVF } \\
(S D)\end{array}$ & $\begin{array}{l}\text { LVF } \\
(S D)\end{array}$ & $\begin{array}{c}\text { BLVF } \\
(S D)\end{array}$ & $\begin{array}{l}\text { RVF } \\
(S D)\end{array}$ & $\begin{array}{c}\text { BRVF } \\
(S D)\end{array}$ \\
\hline Associate & $\begin{array}{c}798.00 \\
(217.00)\end{array}$ & $\begin{array}{c}822.90 \\
(278.21)\end{array}$ & $\begin{array}{c}772.73 \\
(210.29)\end{array}$ & $\begin{array}{c}750.60 \\
(200.36)\end{array}$ & $\begin{array}{c}80.00 \\
(16.66)\end{array}$ & $\begin{array}{c}83.33 \\
(13.47)\end{array}$ & $\begin{array}{l}92.67 \\
(8.00)\end{array}$ & $\begin{array}{r}88.67 \\
(10.00)\end{array}$ \\
\hline Non-Associate & $\begin{array}{c}859.05 \\
(257.16)\end{array}$ & $\begin{array}{c}853.40 \\
(272.43)\end{array}$ & $\begin{array}{c}846.08 \\
(242.59)\end{array}$ & $\begin{array}{c}775.25 \\
(170.00)\end{array}$ & $\begin{array}{c}76.67 \\
(13 . .33)\end{array}$ & $\begin{array}{l}74.67 \\
(9.33)\end{array}$ & $\begin{array}{l}81.33 \\
(5.03)\end{array}$ & $\begin{array}{r}90.67 \\
(14.00)\end{array}$ \\
\hline Non-word & $\begin{array}{c}944.73 \\
(197.87)\end{array}$ & $\begin{array}{c}904.30 \\
(208.23)\end{array}$ & $\begin{array}{c}875.12 \\
(184.04)\end{array}$ & $\begin{array}{c}872.63 \\
(214.13)\end{array}$ & $\begin{array}{c}73.33 \\
(13.33)\end{array}$ & $\begin{array}{c}6.67 \\
(17.33)\end{array}$ & $\begin{array}{c}76.67 \\
(16.66)\end{array}$ & $\begin{array}{c}77.33 \\
(15.33)\end{array}$ \\
\hline
\end{tabular}

Note: Associate $=$ Associated type of stimuli; Non-Associate = Non-associated type of stimuli; Non-word $=$ Non-word stimuli; $B R V F=$ Bilateral right visual field; $\mathrm{RVF}=$ Right visual field $; \mathrm{BLVF}=\mathrm{Bilateral}$ left visual field; $\mathrm{LVF}=$ Left visual field; $\mathrm{LH}=$ Left Hemisphere; $\mathrm{RH}$ : Right hemisphere.

\section{Discussion}

This pilot study was conducted to ensure that the stimuli sets developed for Experiment 3 produced priming effects and did not differ from each other. All participants responded faster and more accurately to the associated pairs than the non-associated pairs, indicating the presence of priming effects. All participants responded faster and more accurately to the targets presented in the RVF than those presented in the LVF, indicating 
the presence of the RVF superiority effect. These findings validated the stimuli sets. Since the RVF superiority effect is a typical finding in a visual half-field presentation paradigm when linguistic stimuli are used (Chiarello, 1985; Clarke \& Zaidel, 1994), the presence of this effect in this pilot study validated the present visual half-field presentation procedure.

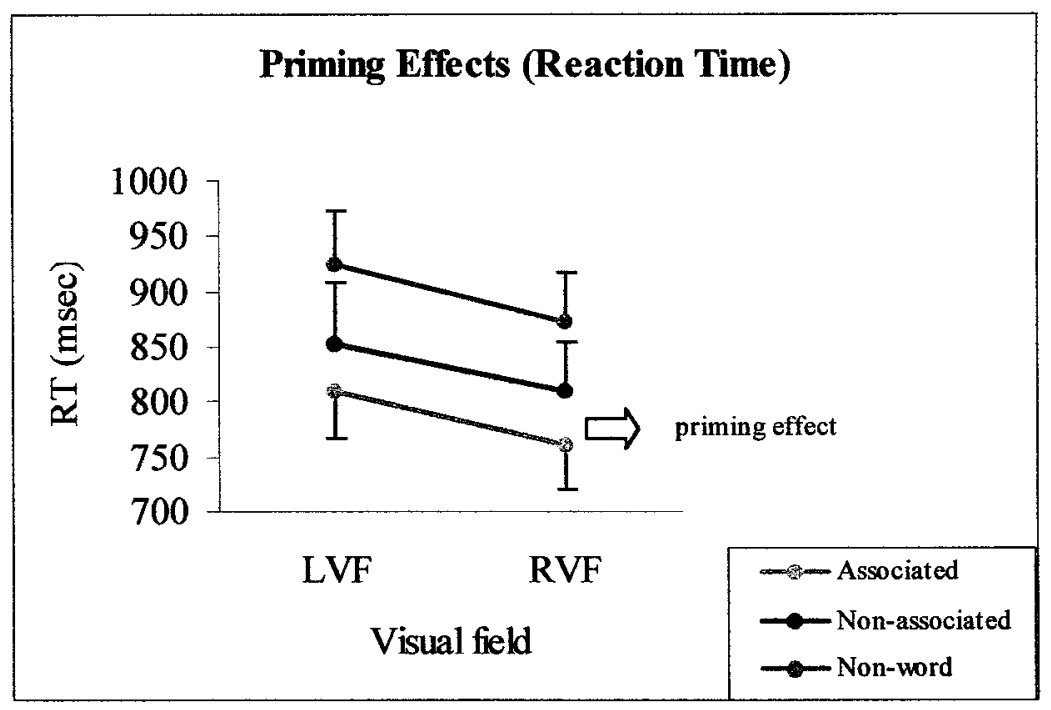

The data are summarized in Figures 1 and 2.

Figure 1. Priming effect in RT. Associated: Associated pairs.

Non-associated: Non-associated pairs. Non-word: Non-word pairs.

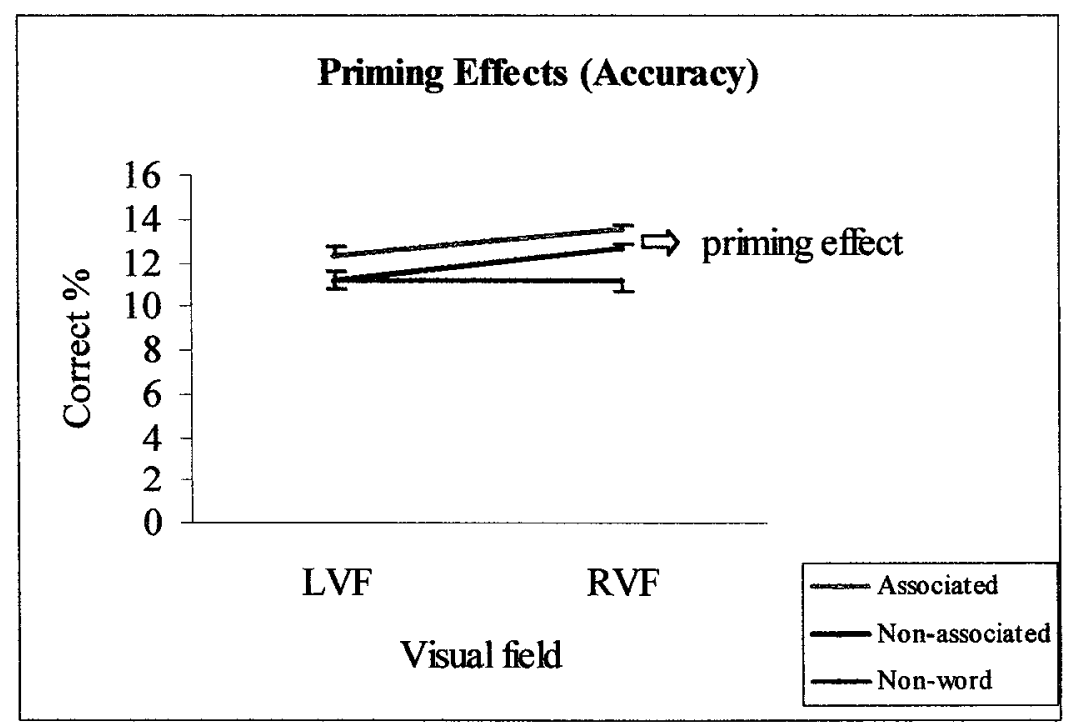


Figure 2. Priming effect in accuracy.

\section{Appendix R}

\section{List of Abbreviations}

\begin{tabular}{|c|c|}
\hline ANOVA & Analysis of Variance \\
\hline $\mathrm{CA}$ & Chronological age-matched \\
\hline CAP & Chronological age-matched poor readers \\
\hline $\mathrm{CC}$ & Corpus callosum \\
\hline $\mathrm{CRH}$ & Consistent right handers \\
\hline CUD & Crossed and Uncrossed reaction time difference \\
\hline CVC & Consonant-Vowel-Consonant \\
\hline DD & Developmental dyslexia \\
\hline DV & Dependent variable \\
\hline$F$ & Fisher's F ratio \\
\hline FLT & Finger localization task \\
\hline GPC & Grapheme-phoneme conversion \\
\hline LD & Learning disabilities \\
\hline LDT & Lexical decision task \\
\hline LGN & Lateral geniculate of the nucleus \\
\hline LH & Left hemisphere \\
\hline LQ & Laterality Quotient \\
\hline LVF & Left visual-field \\
\hline$M$ & Mean \\
\hline
\end{tabular}




\begin{tabular}{ll} 
MANOVA & Multivariate Analysis of Variance \\
Mdn & Median \\
M-system & Magnocellular system \\
nCRH & Non-consistent right handers \\
OCCSB & Ottawa-Carleton Catholic School Board \\
OCSBE & Ottawa-Carleton District School Board \\
PCA & Principal Component Analysis \\
PDE & Phonemic Decoding Efficiency \\
P-system & Parvocellular system \\
PT & Planum temporale \\
$r$ & Pearson product-moment correlation \\
RA & Reading age-matched \\
RH & Right hemisphere \\
RT & Reaction time \\
RVF & Right visual-field \\
SD & Standard deviation \\
SE & Standard errors \\
SS & Standardized score \\
STM & Short term memory \\
SWE & Sight Word Efficiency \\
TONI-3 & Tef of Non-verbal Intelligence-3 \\
& Tord Reading Efficiency \\
\hline &
\end{tabular}


V1

VEP

VF

WM

YA
Primary visual cortex

Visual evoked potential

Visual-field

Working memory

Young Age Group 